\title{
The analysis of advanced glycation endproducts
}

Citation for published version (APA):

Scheijen, J. J. L. J. M. (2017). The analysis of advanced glycation endproducts: a mass spectrometry-based approach \& its applications. [Doctoral Thesis, Maastricht University]. Datawyse / Universitaire Pers Maastricht. https://doi.org/10.26481/dis.20170621js

Document status and date:

Published: 01/01/2017

DOI:

10.26481/dis.20170621js

Document Version:

Publisher's PDF, also known as Version of record

\section{Please check the document version of this publication:}

- A submitted manuscript is the version of the article upon submission and before peer-review. There can be important differences between the submitted version and the official published version of record.

People interested in the research are advised to contact the author for the final version of the publication, or visit the DOI to the publisher's website.

- The final author version and the galley proof are versions of the publication after peer review.

- The final published version features the final layout of the paper including the volume, issue and page numbers.

Link to publication

\footnotetext{
General rights rights.

- You may freely distribute the URL identifying the publication in the public portal. please follow below link for the End User Agreement:

www.umlib.nl/taverne-license

Take down policy

If you believe that this document breaches copyright please contact us at:

repository@maastrichtuniversity.nl

providing details and we will investigate your claim.
}

Copyright and moral rights for the publications made accessible in the public portal are retained by the authors and/or other copyright owners and it is a condition of accessing publications that users recognise and abide by the legal requirements associated with these

- Users may download and print one copy of any publication from the public portal for the purpose of private study or research.

- You may not further distribute the material or use it for any profit-making activity or commercial gain

If the publication is distributed under the terms of Article $25 \mathrm{fa}$ of the Dutch Copyright Act, indicated by the "Taverne" license above, 


\section{The analysis of ADVANCED GLYCATION ENDPRODUCTS A mass spectrometry-based approach \& its applications}

Jean J ohannes Lambertus J oseph M arie Scheijen 
(c) Jean Scheijen, Maastricht, 2017

No part of this book may be reproduced or transmitted in any form or by an means, without prior permission in writing by the author, or when appropriate, by the publishers of the publications.

\section{Paranimfen:}

Ir. D.J.W.M. Scheijen

Bcom. H.W.H.M. Scheijen

Layout \& Cover design: Jean Scheijen | vierdrie.nl

Production: Datawyse | Universitaire Pers M aastricht

ISBN: 9789461597038 


\title{
The analysis of ADVANCED GLYCATION ENDPRODUCTS A mass spectrometry-based approach \& its applications
}

\author{
PROEFSCHRIFT \\ Ter verkrijging van de graad van doctor aan de Universiteit M aastricht, \\ op gezag van de Rector Magnificus, Prof. dr. Rianne M. Letschert, \\ volgens het besluit van het College van Decanen, \\ in het openbaar te verdedigen op \\ woensdag 21 juni 2017 om 16:00 uur
}

door 


\section{Promotores}

Prof. dr. C.G. Schalkwijk

Prof. dr. C.D.A. Stehouwer

\section{Beoordelingscommissie}

Prof. dr. T.M. Hackeng (Voorzitter)

Prof. dr. V. Fogliano (Wageningen University and Research, WUR)

Prof. dr. R.M.A. Heeren

Prof. dr. H.W.M. Niessen (University Medical Center Amsterdam, VUmc)

Prof. dr. A. Opperhuizen (Nederlandse Voedsel en Warenautoriteit, NVWA)

Financial support by the Dutch Heart Foundation for the publication of this thesis is gratefully acknowledged. 


\section{CONTENTS}

$\begin{array}{lll}\text { CHAPTER } 1 \quad \text { General introduction } & 7\end{array}$

CHAPTER 2A Measurement of pentosidine in human plasma protein by

a single-column High-Performance Liquid Chromatography

method with fluorescence detection

CHAPTER 2B M Measurement of advanced glycation endproducts in human plasma by ultra-performance liquid chromatography tandem mass spectrometry

CHAPTER 3 Plasma levels of advanced glycation endproducts $\mathrm{N}^{\varepsilon}$-(carboxymethyl)lysine, $\mathrm{N}^{\varepsilon}$-(carboxyethyl)lysine and pentosidine are not independently associated with cardiovascular disease in individuals with or without type 2 diabetes:

the Hoorn and CODAM Studies

CHAPTER $4 \quad L(+)$ and $D(-)$ Lactate are increased in plasma and urine samples of type 2 diabetes as measured by a simultaneous quantification of $L(+)$ and $D(-)$ Lactate by Reversed Phase Liquid Chromatography Tandem M ass Spectrometry

CHAPTER 5 Higher plasma concentrations of the methylglyoxal metabolite D-lactate are independently associated with insulin resistance: the CODAM study

CHAPTER 6 Quantification of glyoxal, methylglyoxal and 3 deoxyglucosone in blood and plasma by ultra performance liquid chromatography tandem mass spectrometry; evaluation of blood specimen

CHAPTER $7 \quad$ Post-glucose load plasma $\alpha$-dicarbonyl concentrations are increased in individuals with impaired glucose metabolism and type 2 diabetes: The CODAM study

CHAPTER 8 Analysis of advanced glycation endproducts in selected food items by ultra-performance liquid chromatography tandem mass spectrometry; presentation of a dietary AGE database 


\section{CONTENTS}

CHAPTER 9 Dietary intake of advanced glycation endproducts is associated with higher levels of advanced glycation endproducts in plasma and urine: the CODAM study

CHAPTER $10 \quad$ Summary \& general discussion $\quad 185$

$\&$

Samenvatting

Valorization

Curriculum vitae

Scientific output

Dankwoord 233

Foods \& Polaroid 239 



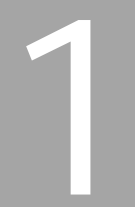

General introduction 


\section{General Introduction}

\section{Diabetes, vascular disease and advanced glycation endproducts}

Age-related, non-communicable chronic inflammatory diseases like cardiovascular disease (CVD) and type 2 diabetes mellitus (T2DM ) represent a major 21st century health problem. The prevalence of these diseases is exponentially rising as the population ages. Diabetes is a common metabolic disease affecting approximately 422 million people worldwide in $2014^{1}$, which is predicted to rise to 600 million in 2040. In the Netherlands, one million people have diabetes, which is predicted to rise to 1.3 million in $2025^{2}$. Diabetes is a serious chronic disease characterized by elevated plasma glucose levels. There are two main types of diabetes: type 1 diabetes mellitus (T1DM) and T2DM . T1DM is characterized by an absolute insulin deficiency as caused by an autoimmune destruction of the pancreatic beta cells. T1DM accounts for approximately $10 \%$ of the prevalence of diabetes ${ }^{3}$. T2DM is characterized by insulin resistance despite of high levels of insulin. This will ultimately result in an absolute defective insulin secretion. T2DM accounts for approximately $90 \%$ of all individuals with diabetes and is primarily caused by lifestyle factors, such as overweight and lack of physical activity ${ }^{3}$. The current obesity epidemic has led to a strong increase in the prevalence of T2DM ${ }^{4}$. The burden of T2DM is mainly determined by macro- and microvascular diseases. Macrovascular disease affects larger blood vessels through atherosclerosis and affects the coronary, the carotid and the peripheral arteries, thus increasing the risk for heart attack, stroke and diabetic foot. Individuals with diabetes are at a 2- to 4-fold increased risk of cardiovascular events compared to non-diabetic individuals ${ }^{5,6}$. CVDs are the number 1 cause of death globally, an estimated 17.5 million deaths were directly caused by CVD in $2012^{7}$. Microvascular disease affects smaller blood vessels of the eyes, kidneys, and nerves ${ }^{8}$ increasing the risk of retinopathy, nephropathy and neuropathy, respectively ${ }^{9}$. Diabetes and its associated complications leads to a permanent and significant loss of quality of life and are acknowledged to be among the highest burden for health care costs ${ }^{10}$. Therefore, prevention of diabetes and in case of diabetes, prevention of cardiovascular disease is of highest importance and clearly represents a medical and economical need. Future strategies should therefore focus primarily on the prevention of diabetes and improved stratification algorithms to allow identification of those who are at risk for developing vascular complications. Early identification of vascular risk is a cornerstone of diabetes management and facilitates tailored intervention at an early stage of disease when a useful response is more likely to be obtained. Given the enormous impact of vascular disease, it is of utmost importance to find biomarkers for the identification of diabetic individuals at high risk of developing vascular complications and morbidity; i.e. to improve risk prediction ${ }^{11}$. So far, the established cardiovascular risk factors and the derived algorithms do not fully explain vascular risk ${ }^{12}$. It is very likely that markers of pathophysiological pathways, which are not covered by the well-known risk factors, will improve risk score. 
Various mechanisms have been proposed to explain how hyperglycaemia directly causes diabetic vascular complications, including the polyol pathway, an increase in glucosamine6-phosphate via the hexosamine pathway, the activation of protein kinase $C$ (PKC) via de novo synthesis of diacylglycerol (DAG) and the non-enzymatic glycation of proteins ${ }^{13}$. The biochemical process of the formation of advanced glycation endproducts (AGEs), which is accelerated in patients with diabetes as a result of elevated plasma glucose levels and increased oxidative stress, has been related to diabetic complications ${ }^{8,13}$. Increased formation and accumulation of AGEs is common in patients with diabetes, and, because of impaired clearance, in particular those with chronic kidney disease $(C K D)^{14}$ or end stage renal disease (ESRD) ${ }^{15}$. The group of AGEs is a heterogeneous family of unavoidable byproducts which are formed by the reaction of proteins with reactive metabolic intermediates derived from glucose and from lipid oxidation with the involvement of oxidative stress ${ }^{16-18}$. AGEs manifest pathological effects which are implicated in the development of age-related disease ${ }^{9,19,20}$. In addition to the overwhelming amount of data demonstrating a role of AGEs in the development of vascular disease ${ }^{9,21-25}$ in individuals with or without diabetes, AGEs are also implicated in the development of insulin resistance and thus the development of diabetes ${ }^{26}$. In addition to endogenous formation of AGEs, it has now become apparent that dietary AGEs represent a significant source of circulating and tissue AGEs ${ }^{27}$.

Thus, increased accumulation of AGEs is a core defect, derived from abnormalities in glucose- and lipid-metabolism, impaired clearance or intestinal absorption, which can lead to diabetes and to vascular complications.

However, so far our knowledge about the putative role of specific AGEs in several diseases is hampered by the lack of good analytical techniques to detect these specific AGEs. In addition, the measurement of AGEs may also help to identify patients with a high risk for poor outcome and may thus help in risk stratification ${ }^{28,29}$. Therefore, the central aim of this thesis is the development and validation of state-of-the-art techniques to quantify precursors of AGEs, AGEs and related compounds and the application of these techniques in biochemical research.

\section{Advanced glycation endproducts and related compounds}

The non-enzymatic reaction of sugars with proteins, better known as the Maillard or browning reaction, leads to formation of $A G E s^{17,30}$. This reaction was described for the first time in 1912 by the chemist Louis Camille Maillard (Figure 1.1), who demonstrated that amino acids heated in the presence of reducing sugars developed a characteristic yellow-brown color ${ }^{31}$. The classical pathway of the Maillard reaction begins with the condensation reaction of the carbonyl group of sugar aldehydes with the $\mathrm{N}$-terminus or free-amino groups of proteins to the formation of a reversible Shiff base (Figure 1.2). This labile adduct undergo rearrangements, through acid-base catalysis, leading to the formation of the more stable Amadori products. These glycation adducts are classified as early glycation products. 
The chemistry of these early glycation products has been well described, with glycated hemoglobin $\left(\mathrm{HbAlc}\right.$ ) as the best-studied example ${ }^{32}$. Only a small part of these stable, but still reversible formed early glycation products, undergo further oxidative reactions to form a variety of irreversible AGEs ${ }^{33}$. One major consequence of the advanced Maillard reaction is the formation of covalently cross-linked proteins which, in long-lived tissues (e.g. skin collagen and lens proteins), accumulate with age ${ }^{18,34,35}$. So far, one of the best characterized lysine-arginine cross-link is the fluorophore pentosidine $e^{35,36}$.

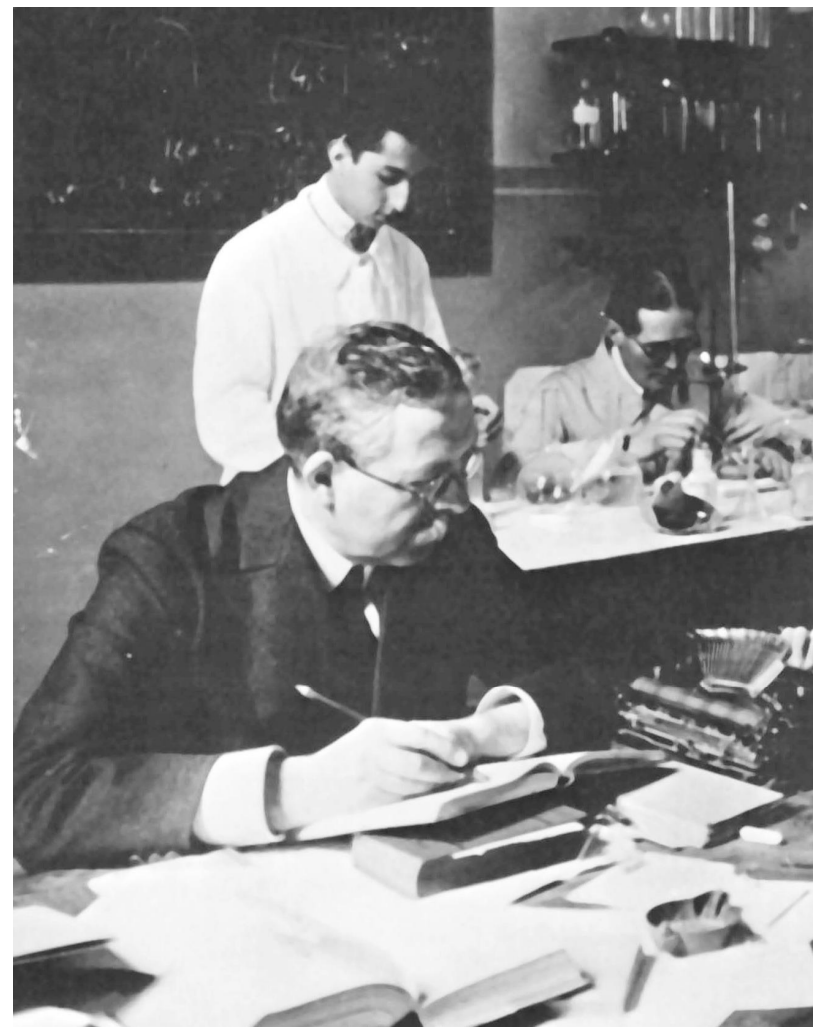

Figure 1.1 Louis Camille M aillard (1878-1936) photographed in his laboratory around 1915

In addition to the classical Maillard reaction we now know that in vivo AGEs are also formed rapidly from highly reactive intermediate dicarbonyl groups, known as oxoaldehydes (or dicarbonyls), including methylglyoxal (MGO), glyoxal (GO) and 3-deoxyglucosone (3-DG) (Figure 1.3) $)^{13}$. Accumulation of these reactive oxoaldehydes is known as "dicarbonyl stress". Many different sources are responsible for the formation of these dicarbonyl metabolites, in particular by increased glucose metabolism but also by lipid peroxidation, degradation of glycated proteins and by intestinal absorption ${ }^{37}$. M GO, the most potent glycation agent, is formed by the non-enzymatic fragmentation of the triose phosphates glyceraldehyde-3-phosphate and dihydroxyacetone phosphate, which are derived from glucose and fructose metabolism. 
This intracellular dicarbonyl compound reacts mainly with arginine to form $\mathrm{N}^{\delta}$-(5-hydro-5methyl-4-imidazolon-2-yl)-ornithine (M G-H1, M G-H2 and M G-H3) ${ }^{38,39}$ and with lysine to form $\mathrm{N}^{\varepsilon}$-(1-carboxyethyl)lysine (CEL). M GO is detoxified by the glyoxalase pathway by two dependent thiol-enzymes; glyoxalase-1 and -2 (GLO-I and GLO-II) and reduced glutathione

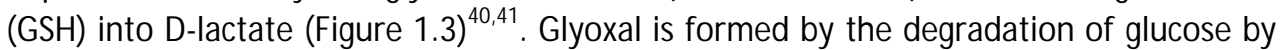
retro-aldol condensation reactions ${ }^{42}$ activated by deprotonation of the 2- and 3-hydroxy groups ${ }^{43}$. Glyoxal reacts further with lysine to form $\mathrm{N}^{\varepsilon}$-(carboxymethyl)lysine (CML), a well-known ligand for the receptor for AGEs (RAGE) ${ }^{44}$. The reduction of glucose to sorbitol and further oxidation to fructose and fructose-3-phosphate leads to the formation of 3-DG. Thus, AGEs can be formed by these sugar-derived carbonyls ${ }^{45}$, by lipid peroxidation and oxidative stress ${ }^{46,47}$ or by reactions between pentoses and proteins, known as the fluorescent lysine-arginine cross-link pentosidine ${ }^{36,48}$.

AGEs, in our body, can also originate from exogenous sources such as diet and smoking ${ }^{49,50}$. AGEs in foods are formed rapidly during processing at high temperatures, such as grilling, frying, baking or roasting ${ }^{49,51,52}$. Since the consumption of these processed foods has increased over the past decades ${ }^{53}$, the exposure to dietary AGEs has also increased and may contribute to the development of chronic diseases ${ }^{27,54,55}$.

It was not until 1980 that the pathophysiological significance of AGEs emerged in medical science $^{17,30}$. So far, accumulation of AGEs in the body, is implicated in the pathogenesis of age-related diseases in particular in relation to diabetes, inflammation, neurodegenerative disorders and cardiovascular disease ${ }^{21,56-59}$. Several mechanisms have been proposed by which AGEs may adversely affect human health; including AGE accumulation in the extracellular matrix proteins, leading to the formation of cross-links and subsequently vascular stiffening, the binding of AGEs to AGE-receptors, consequently activating $\mathrm{NF}_{\mathrm{KB}}$ and MAP-kinase signaling pathways, and cellular dysfunction as a consequence of altered function of proteins by intracellular glycation ${ }^{13,60,61}$.

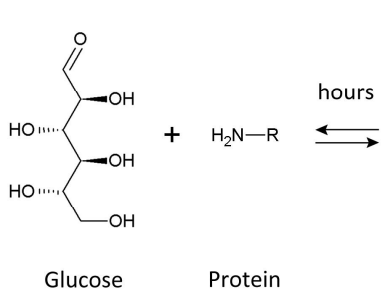

Glucose

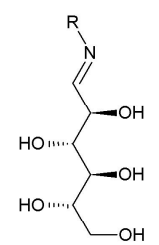

Schiff base

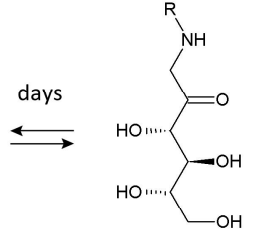

Amadori product

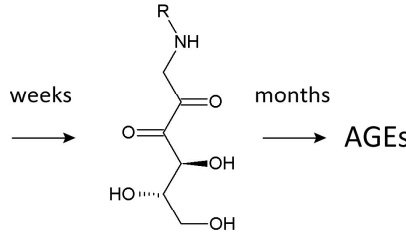

Intermediate glycation product 
(t)

Glyoxal<smiles>CC(NCCCC[C@H](N)C(=O)O)C(=O)O</smiles>

$\mathrm{N}^{\varepsilon}$-(carboxyethyl)lysine (CEL)

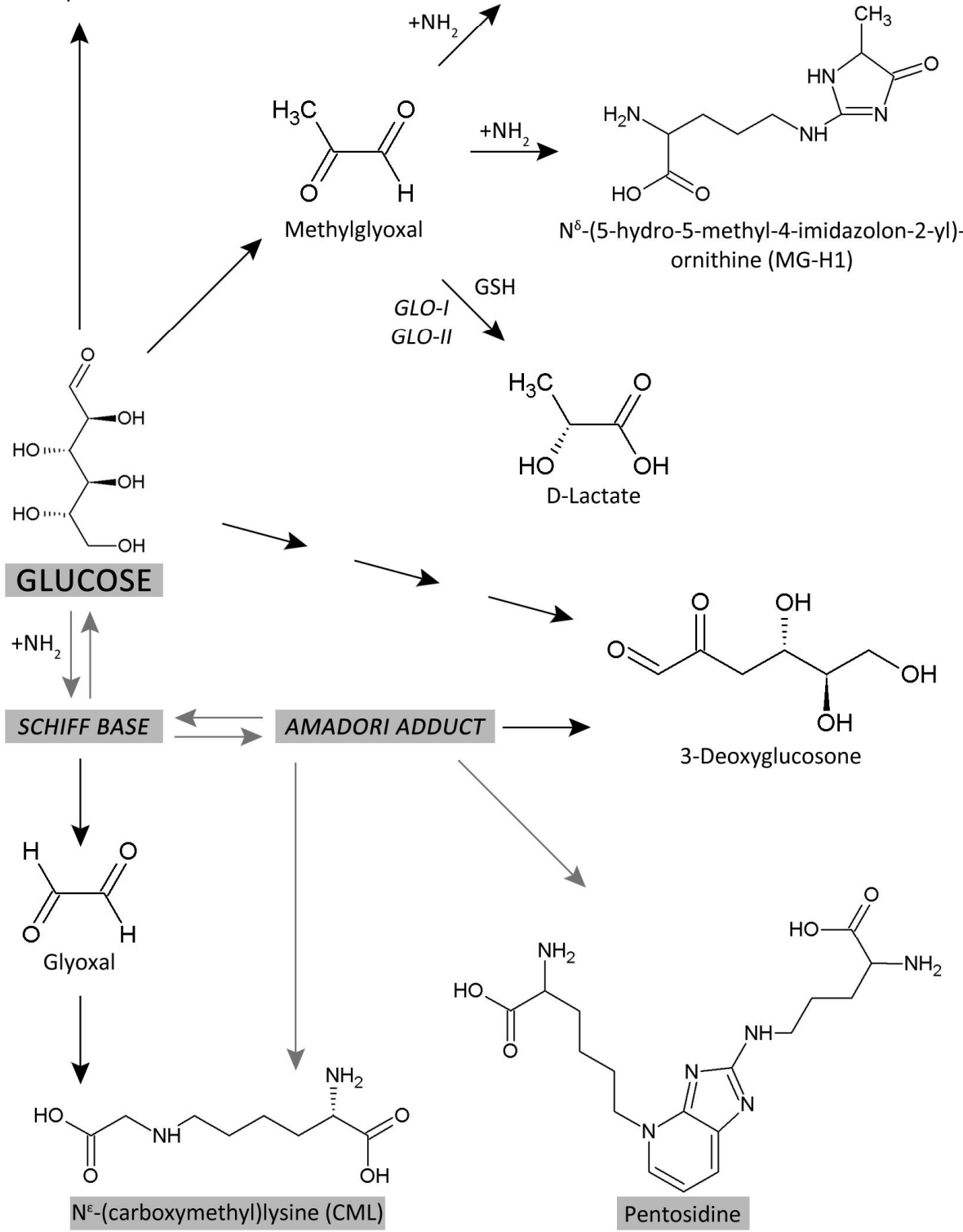

Figure 1.3 The formation of advanced glycation endproducts. 


\section{Analysis of advanced glycation endproducts}

\section{The early beginning of AGE analysis: fluorescence detection}

Since the discovery of AGEs in 1912, by Louis Camille Maillard, it took almost 70 years until the first in vivo analyses were done ${ }^{17,30,62}$. In those days, AGEs were detected as a group of advanced glycation products (AGP) with a brown or fluorescent property. Insoluble human dura mater collagen was digested with collagenase and the absorbance at $350 \mathrm{~nm}$ and fluorescence at $370 / 440 \mathrm{~nm}$ (excitation/emission) of the solubilized material was measured ${ }^{62}$. For the first time AGE accumulation was estimated in long-lived proteins, such as collagen and lens crystallins, and this was closely related to the pathogenesis of aging and diabetes ${ }^{17,30,62}$. More recent, skin autofluorescence $(S A F)^{63}$ is used as a non-invasive tool to assess the AGE accumulation in the skin and can be useful for rapid risk assessment of AGE-related long-term complications in diabetes and related diseases ${ }^{64-67}$. SAF illuminates approximately $1 \mathrm{~cm}^{2}$ skin with an excitation light source of 300 to $420 \mathrm{~nm}$. Autofluorescence was calculated by dividing the average light intensity emitted per nm over the 420 to $600 \mathrm{~nm}$ range by the average light intensity emitted per $\mathrm{nm}$ over the 300 to $420 \mathrm{~nm}$ range ${ }^{63}$. The specificity of these fluorescence techniques is limited because they cannot quantify the concentrations of individual compounds and major AGEs are not fluorescent (e.g. MG-H1, CML and CEL). Moreover, interference of other fluorophores, e.g. nicotinamide adenine dinucleotide (NADH), flavin adenine dinucleotide (FAD), porphyrins, dityrosine and $\mathrm{N}^{\prime}$-formylkynurenine ${ }^{68-70}$, or changes in the absorption of ultraviolet (UV) excitation light due to hemodynamic and oximetric effects ${ }^{71}$ may influence the relationship between (auto)fluorescence and AGE. Therefore, the interpretation of SAF data, as an estimate of AGEs, and the relationship with disease should be interpreted with care. Nevertheless, SAF can be used as a marker of the total skin AGE pool and was linked to age, renal failure and is closely related to risk-factors for long-term diabetic complications ${ }^{66,67,72}$.

\section{The next step in AGE analysis: immunochemical detection}

To get a better understanding of the pathological consequences of AGEs a more specific technique was needed. In the beginning of 1990, Nakayama ${ }^{73}$, Makita $^{74}$, Horiuchi $^{75}$ and co-workers described immunochemical approaches using antibodies specific for AGEs on proteins. Mono- and polyclonal anti- antibodies against AGE-epitopes were raised and AGE measurements were performed with an enzyme-linked immunosorbent assay (ELISA). Although immunoassays can be applied as a high throughput, simple and cheap method to quantify AGEs, it remained difficult to produce reliable and reproducible results. In the following years ELISA-based techniques were improved and several antibodies against mainly CML-modified proteins were developed and used for AGE analysis $^{76-80}$. However, quantification of AGEs with ELISA-based techniques did not give satisfying results. The antibody $6 \mathrm{D} 12$, which was widely applied to quantify $\mathrm{CM} L$, showed cross-reaction to CEL, thus indicating the lack of epitope specificity ${ }^{81,82}$. 
Moreover, the use of blocking proteins, such as serum albumin and gelatin, to decrease the non-specific binding of primary and secondary antibodies, gave misleading results because of the presence of glycated amino acids in these blocking proteins ${ }^{83}$. Also, preanalytic processing such as the use of extreme high $\mathrm{pH}$ or heating, degrade $\mathrm{N}^{\varepsilon}$-fructosyllysine residues rapidly to form $\mathrm{AGEs}^{84,85}$. Indeed, most recent papers on the comparison of ELISA-based techniques with instrumental techniques, such as gas chromatography mass spectrometry (GC-MS) ${ }^{86,87}$ and liquid chromatography tandem mass spectrometry $(\mathrm{LC}-\mathrm{MS} / \mathrm{MS})^{87,88}$, revealed inconsistent results. We have compared the CML content in food items, as analyzed with our UPLC-MS/MS technique with results from an ELISA based technique, as described by Uribarri et al. ${ }^{89}$ and with a UPLC-M S/MS technique, as reported by Hull et al. ${ }^{90}$ (Figure 1.4). In 29 food items, based on the same description and preparation of each food item, we have measured the CML content and found no significant correlation between our UPLC-MS/MS and the ELISA technique ( $r=0.32, N . S$, $p=0.74)$. However, a significant correlation was found between both UPLC-MS/MS techniques $(r=0.82, p \varangle 0.0001)$ (Figure 1.4).

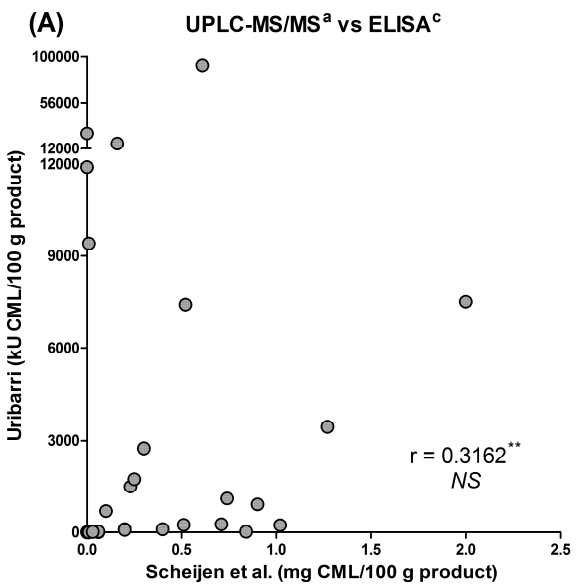

(B) UPLC-MS/MS ${ }^{a}$ vs UPLC-MS/MS ${ }^{b}$

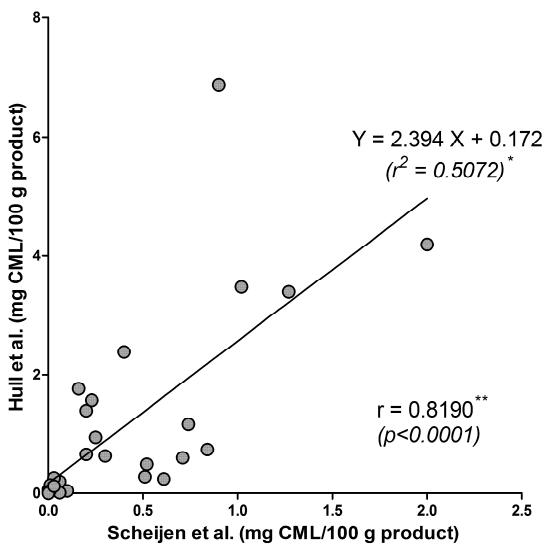

Figure 1.4. Comparison of CML content in selected food items analyzed with different techniques.

(A) Correlation of CML content in 29 food items as analyzed with our UPLC-MS/MS technique and ELISA described by Uribarri et al. (Uribarri et al., 2010)

(B) Correlation of CML content in 29 food items as analyzed with our UPLC-MS/MS technique and the UPLCMS/MS technique described by Hull et al. (George L.J. Hull et al., 2012)

(* $r$-square based on linear regression, $* * r$ based on Spearman correlation, NS $=$ not significant $(p>0.05))$.

${ }^{a} \mathrm{CM} L$ content in food items based on UPLC-M S/M S analysis by Scheijen et al.

${ }^{b} \mathrm{CML}$ content in food items based on UPLC-M S/M S analysis by Hull et al. (George L.J. Hull, 2012)

${ }^{c} \mathrm{CM} L$ content in food items based on ELISA analysis by Uribarri et al. (Uribarri et al., 2010)

Though, an advantage of specific antibodies is the use of immunohistochemistry to stain tissues, such as brain tissue ${ }^{91}$, atherosclerotic lesions ${ }^{92}$ and aortic tissues ${ }^{93}$. This technique is widely used to study the distribution and localization of specific AGEs in biological tissues. It is a simple, highly sensitive, specific and semi-quantitative technique for the detection of AGEs in many different tissues. Thus, many estimates of AGEs were done with immunochemical methods but numerous problems have arisen and therefore AGE analysis on a molecular level with analytical techniques is required. 


\section{INTERM EZZO}

\section{History of chromatography}

In 1903, chromatography was invented by the Russian botanist Mikhail Semyonovich Tsvet ${ }^{94-96}$ and coined the word chromatography which is coming from the Greek word chroma meaning color and graphein meaning writing ${ }^{95}$. He used column chromatography to study pigments in plants and one of his first methods was published in $1906^{97}$. It took until the year 1930 before Tsvet's work found his attention in the scientific world. In 1931, Kuhn, Winterstein and Lederer published their method to study carotene-like compounds and Reichstein used the same technique to isolate corticosteroids ${ }^{94}$. Liquid chromatography, as we know it today, was used for the first time in 1941 by Archer John Porter M artin and Richard Laurence M illington Synge ${ }^{98}$. They used liquid-liquid extraction chromatography to study the amino acid composition of wool. In 1952, the Nobel Prize in Chemistry was awarded jointly to Martin and Synge for their invention of partition chromatography ${ }^{99}$. Further development of liquid chromatography to the modern and general purpose high performance liquid chromatography (HPLC) system was done at the early 1960s by Csaba Horvát and Josef Huber ${ }^{100}$. It was in the late 1960 s that firms like Waters Associates and DuPont introduced commercial HPLC equipment and this leaded to the first HPLC paper in $1966^{101}$. The number of HPLC-related publications grew from that year on exponentially ${ }^{100}$.

\section{Ultra performance liquid chromatography}

Since the introduction of the general purpose HPLC system, many manufacturers worked on the improvement of different parts, such as better pumps, injectors and detectors of the HPLC system. Besides these developments there were other fundamental improvements needed to enable HPLC to become a leading analytical technique. The evolution of the packing materials, particularly the decrease of the particle size, improved column efficiency to new limits ${ }^{102}$.

To investigate column efficiency, van Deemter stated an empirical formula describing the relationship between linear velocity $(\mathrm{u}, \mathrm{mm} / \mathrm{s})$ and plate height $(\mathrm{H}, \mu \mathrm{m})$, the so-called van Deemter $\mathrm{H}$-u curve (Figure 1.5) ${ }^{103}$. With this equation it was possible to compare column performance between columns packed with different particle size. Small plate heights indicate higher efficiency and subsequently narrow peaks. As from the introduction of HPLC in the late 1960s, column particles decreased from $10 \mu \mathrm{m}$ in the 1970s, to $5 \mu \mathrm{m}$ in the $1980 \mathrm{~s}$, to $3.5 \mu \mathrm{m}$ in the $1990 \mathrm{~s}$ and to $1.7 \mu \mathrm{m}$ in $2004^{102}$. By using this smaller particle size, a significant gain in efficiency was reached (Figure 1.5). Not only faster chromatography was achieved but also better resolution and increased sensitivity, due to sharper and higher peaks ${ }^{104}$. With the introduction of $1.7 \mu \mathrm{m}$ particles column efficiency was no longer dependent on flow rate or linear velocity (Figure 1.5) 


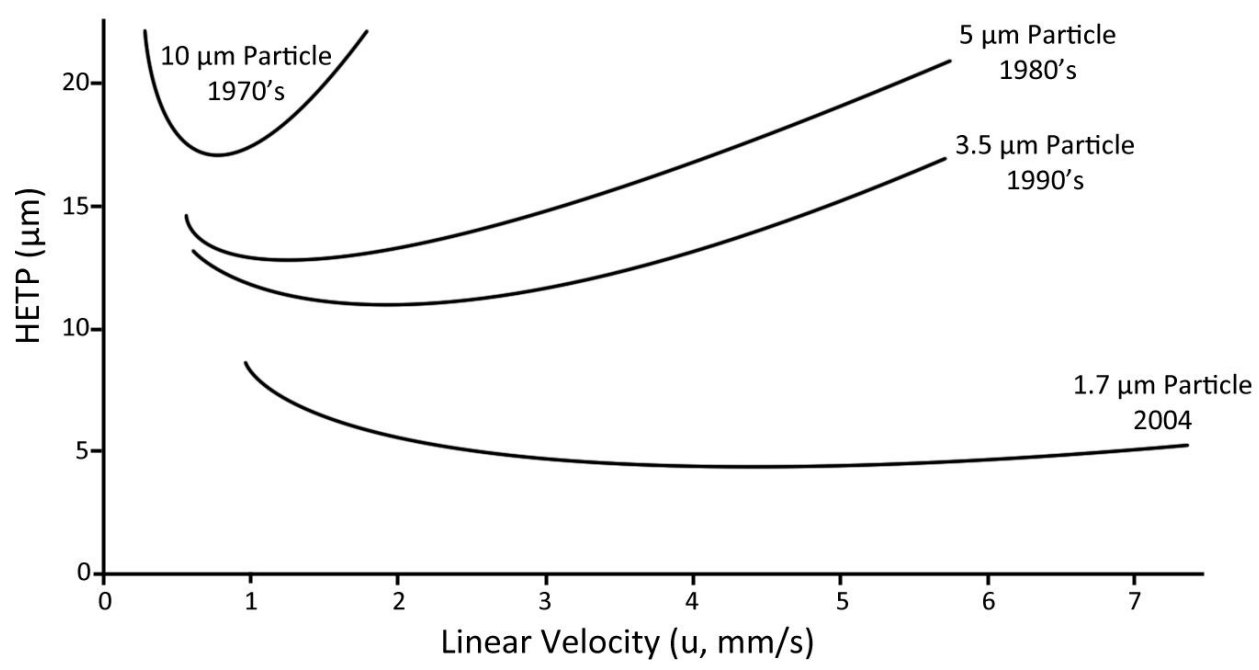

Figure 1.5 Van Deemter H-u curve, evolution of particle size and column efficiency.

\section{High sensitive and specific detection of biomolecules}

Since the introduction of HPLC and UPLC in biomolecule analysis, a highly specific and sensitive detection method was needed. Mass spectrometry is one of these detection techniques, used for the first time in 1897 by Sir Joseph John Thomson ${ }^{105}$ and further expanded by Wolfgang Paul ${ }^{106}$, M alcolm Dole ${ }^{107}$ and John Benett Fenn ${ }^{108}$ and led to more than 7700 methodological publications in 2016 (figure 1.6).

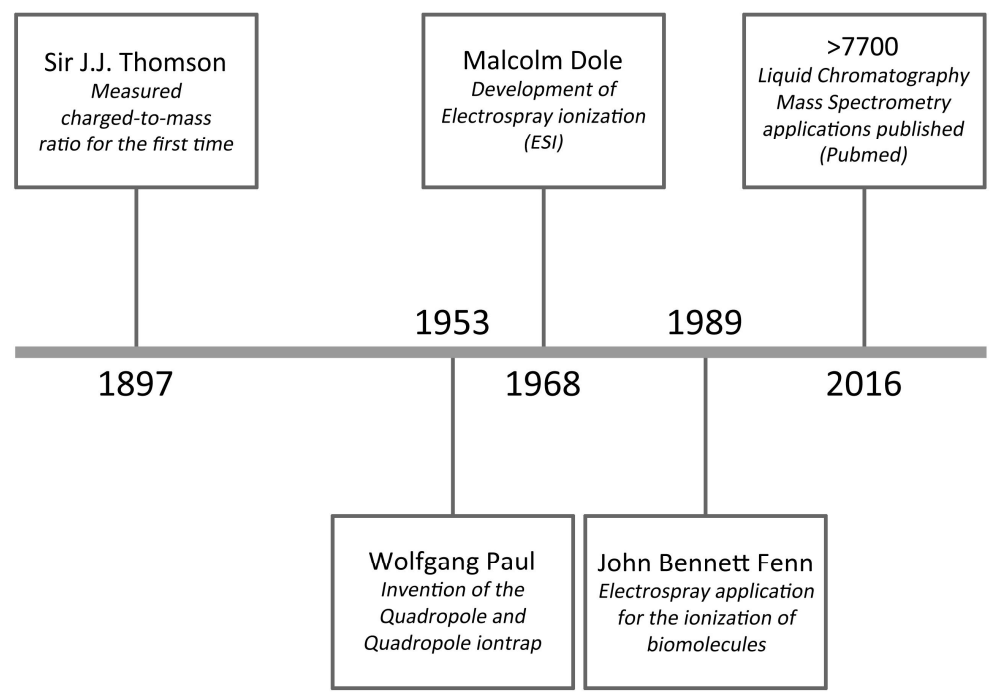

Figure $\mathbf{1 . 6}$ Important $20^{\text {th }}$-century developments in liquid chromatography mass spectrometry. 
From the 1990s onwards, several key developments have made M S one of the strongest tools in biomarker quantification and discovery. Tandem mass spectrometry (MS/MS) was one of these important developments, introduced in 1968 by Professor Keith R. Jennings and Professor Fred M cLafferty ${ }^{109}$. A tandem MS, also known as a triple quadrupole MS, consists of two MS stages with a fragmentation cell in between the two stages (figure 1.7). Ions are formed in the ion source and one specific ion (precursor or parent ion) is selected by the first stage (MS1). Next, this parent ion is fragmented by collision induced dissociation with argon atoms creating fragment ions (daughter ions) which are selected in the second stage of the MS (MS2) (figure 1.7).

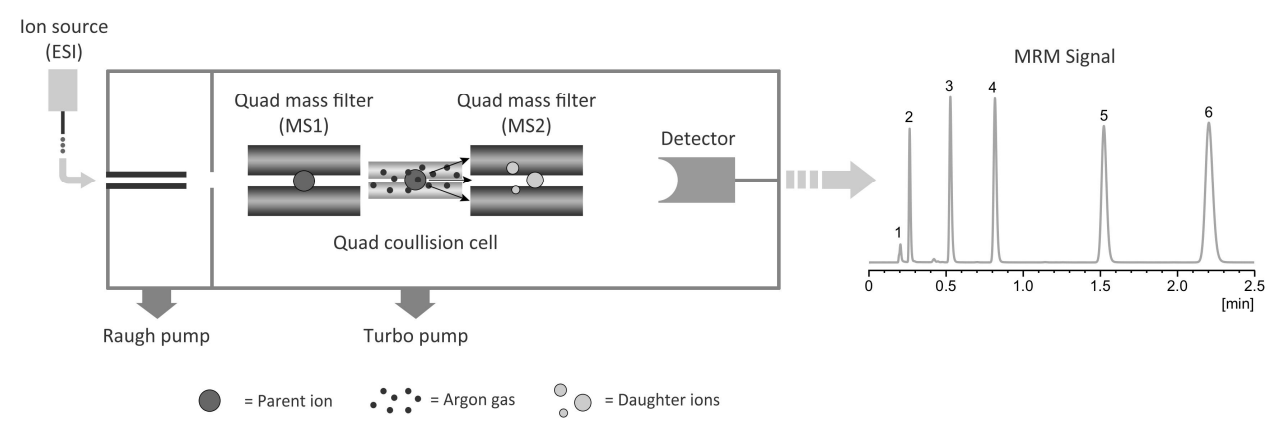

Figure 1.7 Schematic view Tandem mass spectrometer.

By selecting the most abundant fragment ion a multiple reaction monitoring (M RM) transition is made and in this way a highly specific detection is possible. Each compound, including its corresponding stable isotope, is characterized by its own chromatographic retention time, parent ion $(\mathrm{m} / \mathrm{z}$ ) and daughter ion $(\mathrm{m} / \mathrm{z})$. Quantification of the compounds of interest is performed by calculating the peak area ratio of each unlabeled peak area to the corresponding internal standard (stable isotope) peak area.

UPLC-MS/MS analysis provides reliable and robust quantitative data with very small amounts of sample (varying from 20 to $30 \mu \mathrm{L}$ plasma or urine) and is applicable for a broad range of biomolecules. Application of a fully-programmable auto-injector makes it possible to analyze $>100$ samples per day and is therefore very useful for studying disease in large cohort studies. From 1981 until 2015 an exponential increase in LC-MS/MS publications has been seen and underlines the significance of this technique to quantify a broad range of analytes (figure 1.8). 


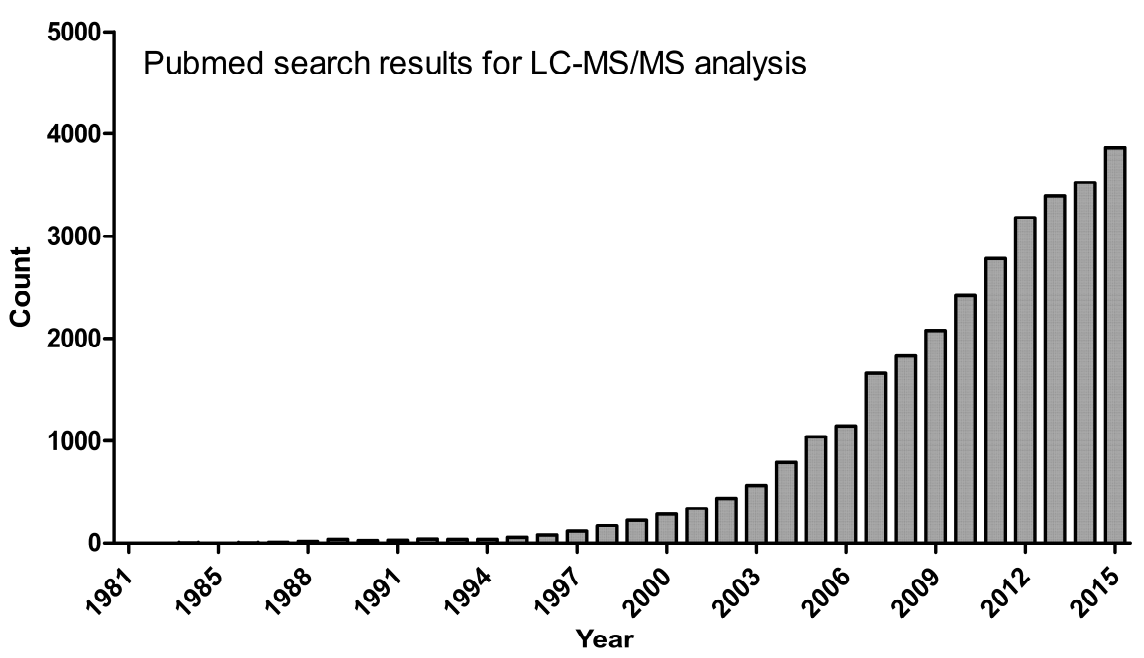

Figure $\mathbf{1 . 8}$ Increase of LC-M S/M S applications as published over the years.

\section{The gold standard in AGE analysis: mass spectrometry detection}

Quantification of AGEs with instrumental methods, such as liquid chromatography (LC) or gas chromatography $(\mathrm{GC})$, needs a completely different sample preparation compared to immunochemical techniques. Free (modified) amino acids are needed in case of the instrumental methods whereas intact (glycated) proteins can be used for immunochemical techniques. To obtain these free (modified) amino acids, the peptide bonds of the intact protein and peptides have to be hydrolyzed. Two hydrolyzation methods have been described, namely by hydrochloric acid ${ }^{88,110}$ and by enzymatic digestion ${ }^{39,111}$. Acid hydrolysis is performed by heating the protein (pellet) with $6 \mathrm{~mol} / \mathrm{L}$ hydrochloric acid for 24 hours at a temperature of $110^{\circ} \mathrm{C}$ in sealed vials. This way of hydrolyzation is cheap, easy and is the most reliable procedure to obtain free amino acids. Prior to this hydrolysis it is necessary to perform a reduction step with sodium borohydride to prevent formation of CM L out of early glycation products during heating in hydrochloric acid ${ }^{112}$. However, some AGEs, such as hydroimidazolones and pyrralines are acid unstable, and therefore enzymatic hydrolysis has been used ${ }^{113}$. For the compounds that are acid unstable and could not be adjusted for the decrease after acid hydrolysis, as we have demonstrated for MG-H1 (Chapter 2B), enzymatic hydrolysis is an alternative. Enzymatic hydrolysis is carried out using a cocktail of endo- and exopeptidases, which cleaves the peptide bonds within and at the end of the protein, respectively. A mixture of pepsin, pronase $\mathrm{E}$, prolidase and aminopeptidase was used for many different purposes ${ }^{113-115}$. However, the recovery of the release of free amino acids by this enzymatic hydrolysis remains questionable. Due to blockage of the cleavage sites by modified arginine and lysine residues, inhibition of the protease by some glycation adducts and lack of cleavage due to side chain modifications, enzymatic hydrolysis may lead to an underestimation of AGE content ${ }^{116,117}$. Therefore, in case of acid stable analysis of AGEs, the most preferable hydrolyzation technique is acid hydrolyzation. 
Acid hydrolyzation has been widely used to hydrolyze plasma and food matrices to quantify AGEs with instrumental methods, including GC-M S $S^{86,118}$ and LC-M S $S^{52,110,119}$. Purification and separation of AGEs from the broad range of other polar compounds, in plasma and food sample hydrolysates, is of utmost importance before detecting them with these MS techniques. In particular, avoiding quenching of the compounds of interest, when using electrospray ionization (ESI) is challenging ${ }^{120,121}$. With the introduction of ultra-performance liquid chromatography (UPLC) in 2004, a significant gain in resolution and thus separation of complex matrices was achieved ${ }^{102}$. The use of UPLC in combination with stable-isotope dilution and subsequently detection with ESI multiple reaction monitoring (M RM) is the best available analytical technique to quantify AGEs.

Besides the use of a state-of-the-art technique, such as UPLC-MS/MS, to quantify AGES and other biomolecules, derivatization of the compounds of interest could increase sensitivity and selectivity. A broad range of derivatization reagents have therefore been used to detect biomolecules with MS techniques, including 1-butanol to derivatize amino acids $^{122}$, diacetyl-L-tartaric anhydride (DATAN) to derivatize glutaric acids ${ }^{123}$ and 0 -phenlylenediamine (OPD) to derivatize $\alpha$-oxoaldehydes ${ }^{124}$. These three reagents have been used in this thesis to separate and quantify butylated AGEs, enantiomeric L- and D-lactate and $\alpha$-oxoaldehydes as quinoxaline derivatives with ESI-UPLC-M S/M S. AGEs and other related compounds were detected and quantified by stable isotope dilution UPLC-MS/MS. Plasma, urine or food samples were prepared according to the physical properties of the compound of interest and sample matrix. Deproteinization and subsequently derivatization of the dried supernatant, in case of the free adducts, and hydrolyzation of the protein-pellet and subsequently derivatisation of the dried hydrolysate, in case of the protein-bound adducts, was applied before analysis.

Derivatives, and their corresponding stable isotopes, were first separated with UPLC and then ionized with ESI-positive mode and detected by M RM.

\section{Aims and outline of this thesis}

Specific, sensitive and reliable techniques are needed to quantify a broad spectrum of well-characterized AGEs. Since the introduction of HPLC in the late 1960s and further development to UPLC in 2004 this state-of-the-art technique came available. The aim of this thesis is to develop and validate several liquid chromatography techniques for the detection of precursors of AGEs and AGEs as well as an application of each technique.

Chapter 2A describes a rapid and sensitive single-column HPLC method for the quantification of protein-bound pentosidine with fluorescence detection. With this technique, we have measured plasma levels of protein-bound pentosidine in uremic patients on peritoneal dialysis and compared to healthy controls. 
Chapter 2B describes a sensitive, simple and highly selective method to determine protein-bound and free $\mathrm{CM} \mathrm{L,} \mathrm{CEL} \mathrm{and} \mathrm{M} \mathrm{G-H1} \mathrm{in} \mathrm{plasma} \mathrm{by} \mathrm{UPLC-tandem} \mathrm{MS.} \mathrm{Plasma} \mathrm{free}$ and protein-bound AGEs were measured in healthy volunteers and patients on peritoneal dialysis.

In Chapter 3, the association of the above mentioned plasma AGEs with prevalent CVD was investigated in individuals with various degrees of glucose metabolism. Plasma levels of AGEs were quantified using both methods as described in Chapter $2 \mathrm{~A}$ and $\mathrm{B}$.

$D$-lactate is the degradation product of methylglyoxal by the glyoxalase pathway. Therefore, D-lactate can be used as a reflection of methylglyoxal. In Chapter 4, the quantification of D- and L-lactate using UPLC-tandem MS without the use of chiral stationary phase chromatography was described. D- and L-lactate were analyzed in plasma and urine of controls, patients with inflammatory bowel disease and patients with T2DM.

In Chapter 5, the association between plasma D-lactate and insulin resistance was investigated.

Chapter 6 describes a method for the quantification of the $\alpha$-oxoaldehydes; methylglyoxal, glyoxal and 3-deoxyglucosone with use of UPLC-tandem MS. An improved sample preparation is evaluated and increased plasma levels of T2DM patients were found as compared to non-diabetic controls.

In Chapter 7, plasma levels of $\alpha$-oxoaldehydes were investigated after a glucose load in individuals without and with impaired glucose metabolism and T2DM patients.

Chapter $\mathbf{8}$ describes a validation of an UPLC-tandem MS method for the determination of AGEs in food items and presents a database of dietary AGEs in a selection of food items commonly consumed in a Western diet.

In Chapter 9, the association of dietary AGEs with plasma and urinary AGEs was investigated.

The results of this thesis are summarized and discussed in Chapter $\mathbf{1 0 .}$ 


\section{References}

WHO. Diabetes: Fact sheet no. 312, (2015).

Baan, C. A. et al. [Diabetes mellitus in the Netherlands: estimate of the current disease burden and prognosis for 2025]. Ned Tijdschr Geneeskd 153, A580 (2009).

American Diabetes, A. Diagnosis and classification of diabetes mellitus. Diabetes care $\mathbf{2 7}$ Suppl 1, S5S10 (2004).

$$
\text { WHO. Global report on diabetes, (2016). }
$$

Sarwar, N. et al. Diabetes mellitus, fasting blood glucose concentration, and risk of vascular disease: a collaborative meta-analysis of 102 prospective studies. Lancet 375, 2215-2222, doi:10.1016/s01406736(10)60484-9 (2010).

$$
\text { Fox, C. S. et al. Trends in cardiovascular complications of diabetes. Jama 292, 2495-2499, }
$$
doi:10.1001/jama.292.20.2495 (2004).

\section{WHO. Diabetes: Fact sheet no. 310, (2014).}

Diagnosis and classification of diabetes mellitus. Diabetes Care 27 Suppl 1, S5-S10 (2004).

Hanssen, N. M. et al. Higher levels of advanced glycation endproducts in human carotid atherosclerotic plaques are associated with a rupture-prone phenotype. Eur Heart J 35, 1137-1146, doi:10.1093/eurheartj/eht402 (2014).

da Rocha Fernandes, J. et al. IDF Diabetes Atlas estimates of 2014 global health expenditures on diabetes. Diabetes Res Clin Pract 117, 48-54, doi:10.1016/j.diabres.2016.04.016 (2016).

Herder, C., Karakas, M. \& Koenig, W. Biomarkers for the prediction of type 2 diabetes and cardiovascular disease. Clinical pharmacology and therapeutics 90, 52-66, doi:10.1038/clpt.2011.93 (2011).

Erhardt, L., Moller, R. \& Puig, J. G. Comprehensive cardiovascular risk management--what does it mean in practice? Vascular health and risk management 3, 587-603 (2007).

Brownlee, M. Biochemistry and molecular cell biology of diabetic complications. Nature 414, 813-820 (2001).

Stinghen, A. E., Massy, Z. A., Vlassara, H., Striker, G. E. \& Boullier, A. Uremic Toxicity of Advanced Glycation End Products in CKD. J Am Soc Nephrol 27, 354-370, doi:10.1681/asn.2014101047 (2016).

Suliman, M. E. et al. Plasma pentosidine is associated with inflammation and malnutrition in endstage renal disease patients starting on dialysis therapy. J Am Soc Nephrol 14, 1614-1622 (2003).

Brownlee, M. Advanced protein glycosylation in diabetes and aging. Annual review of medicine 46, 223-234, doi:10.1146/annurev.med.46.1.223 (1995).

7 Brownlee, M., Vlassara, H. \& Cerami, A. Nonenzymatic glycosylation and the pathogenesis of diabetic complications. Annals of internal medicine 101, 527-537 (1984).

Monnier, V. M. Nonenzymatic glycosylation, the Maillard reaction and the aging process. Journal of gerontology 45, B105-111 (1990).

9 Strozecki, P. et al. Advanced glycation end products and arterial stiffness in patients with diabetic nephropathy and patients with chronic kidney disease without diabetes. Polskie Archiwum Medycyny Wewnetrznej 123, 609-616 (2013).

Sourris, K. C. et al. Plasma advanced glycation end products (AGEs) and NF-kappaB activity are independent determinants of diastolic and pulse pressure. Clin Chem Lab Med 52, 129-138, doi:10.1515/cclm-2012-0850 (2014). 
Baumann, M. et al. N epsilon-(carboxymethyl)|ysine during the early development of hypertension. Ann N Y Acad Sci 1126, 201-204 (2008).

Hartog, J. W. et al. Clinical and prognostic value of advanced glycation end-products in chronic heart failure. Eur Heart ) 28, 2879-2885 (2007).

Lieuw, A. F. M. L. et al. Increased levels of N(epsilon)-(carboxymethyl)lysine and N(epsilon)(carboxyethyl)lysine in type 1 diabetic patients with impaired renal function: correlation with markers of endothelial dysfunction. Nephrol Dial Transplant 19, 631-636 (2004).

Nin, J. W. et al. Higher plasma levels of advanced glycation end products are associated with incident cardiovascular disease and all-cause mortality in type 1 diabetes: a 12-year follow-up study. Diabetes Care 34, 442-447, doi:10.2337/dc10-1087 (2011).

5

Verzijl, $\mathrm{N}$. et al. Crosslinking by advanced glycation end products increases the stiffness of the collagen network in human articular cartilage: a possible mechanism through which age is a risk factor for osteoarthritis. Arthritis and rheumatism 46, 114-123, doi:10.1002/15290131(200201)46:1<114::aid-art10025>3.0.c0;2-p (2002).

Teichert, T. et al. Association between Advanced Glycation End Products and Impaired Fasting Glucose: Results from the SALIA Study. PLoS One 10, e0128293, doi:10.1371/journal.pone.0128293 (2015).

Clarke, R. E., Dordevic, A. L., Tan, S. M., Ryan, L. \& Coughlan, M. T. Dietary Advanced Glycation End Products and Risk Factors for Chronic Disease: A Systematic Review of Randomised Controlled Trials. Nutrients 8, doi:10.3390/nu8030125 (2016).

Kilhovd, B. K. et al. High serum levels of advanced glycation end products predict increased coronary heart disease mortality in nondiabetic women but not in nondiabetic men: a population-based 18year follow-up study. Arterioscler Thromb Vasc Biol 25, 815-820 (2005).

Koyama, Y. et al. High serum level of pentosidine, an advanced glycation end product (AGE), is a risk factor of patients with heart failure. J Card Fail 13, 199-206 (2007).

Monnier, V. M. \& Cerami, A. Nonenzymatic browning in vivo: possible process for aging of long-lived proteins. Science 211, 491-493 (1981).

Maillard, L. C. Action des acides aminés sur les sucres: formation des mélanoidines par voie méthodique. C.R. Acad. Sci. 154, 66-68 (1912).

Gorst, C. et al. Long-term Glycemic Variability and Risk of Adverse Outcomes: A Systematic Review and M eta-analysis. Diabetes Care 38, 2354-2369, doi:10.2337/dc15-1188 (2015).

Schalkwijk, C. Therapeutic Interventions in the Glyc(oxid)ation Pathway. Immun., Enoc. \& Metab. Agents in M ed. Chem. 7, 57-68 (2007).

Monnier, V. M., Genuth, S. \& Sell, D. R. The pecking order of skin Advanced Glycation Endproducts (AGEs) as long-term markers of glycemic damage and risk factors for micro- and subclinical macrovascular disease progression in Type 1 diabetes. Glycoconjugate journal 33, 569-579, doi:10.1007/s10719-016-9702-2 (2016).

Dyer, D. G. et al. Accumulation of Maillard reaction products in skin collagen in diabetes and aging. J Clin Invest 91, 2463-2469, doi:10.1172/jci116481 (1993).

6 Dyer, D. G., Blackledge, J. A., Thorpe, S. R. \& Baynes, J. W. Formation of pentosidine during nonenzymatic browning of proteins by glucose. Identification of glucose and other carbohydrates as possible precursors of pentosidine in vivo. J Biol Chem 266, 11654-11660 (1991).

7 Rabbani, N. \& Thornalley, P. J. Dicarbonyl stress in cell and tissue dysfunction contributing to ageing and disease. Biochem Biophys Res Commun 458, 221-226, doi:10.1016/j.bbrc.2015.01.140 (2015). 
Chen, S. J., Aikawa, C., Yoshida, R. \& Matsui, T. Methylglyoxal-derived hydroimidazolone residue of plasma protein can behave as a predictor of prediabetes in Spontaneously Diabetic Torii rats. Physiological reports 3, doi:10.14814/phy2.12477 (2015).

Ahmed, N., Argirov, O. K., Minhas, H. S., Cordeiro, C. A. \& Thornalley, P. J. Assay of advanced glycation endproducts (AGEs): surveying AGEs by chromatographic assay with derivatization by 6aminoquinolyl-N-hydroxysuccinimidyl-carbamate and application to Nepsilon-carboxymethyl-lysineand Nepsilon-(1-carboxyethyl)lysine-modified albumin. Biochem J 364, 1-14 (2002).

Maessen, D. E., Stehouwer, C. D. \& Schalkwijk, C. G. The role of methylglyoxal and the glyoxalase system in diabetes and other age-related diseases. Clin Sci (Lond) 128, 839-861, doi:10.1042/cs20140683 (2015).

Schalkwijk, C. G. Vascular AGE-ing by methylglyoxal: the past, the present and the future. Diabetologia 58, 1715-1719, doi:10.1007/s00125-015-3597-5 (2015). Huyghues-Despointes, A. \& Yaylayan, V. A. Retro-Aldol and Redox Reactions of Amadori Compounds: Mechanistic Studies with Variously Labeled D-[13C]Glucose. J. Agric. Food Chem. 44, 672-681 (1996). Thornalley, P. J., Langborg, A. \& Minhas, H. S. Formation of glyoxal, methylglyoxal and 3deoxyglucosone in the glycation of proteins by glucose. Biochem J 344 Pt 1, 109-116 (1999). Kislinger, T. et al. N(epsilon)-(carboxymethyl)lysine adducts of proteins are ligands for receptor for advanced glycation end products that activate cell signaling pathways and modulate gene expression. The Journal of biological chemistry 274, 31740-31749 (1999).

Giardino, I., Edelstein, D. \& Brownlee, M. Nonenzymatic glycosylation in vitro and in bovine endothelial cells alters basic fibroblast growth factor activity. A model for intracellular glycosylation in diabetes. The Journal of clinical investigation 94, 110-117, doi:10.1172/JCl117296 (1994).

Gaens, K. H., Stehouwer, C. D. \& Schalkwijk, C. G. Advanced glycation endproducts and its receptor for advanced glycation endproducts in obesity. Curr Opin Lipidol 24, 4-11, doi:10.1097/M OL.0b013e32835aea13 (2013).

Fu, M. X. et al. The advanced glycation end product, Nepsilon-(carboxymethyl)lysine, is a product of both lipid peroxidation and glycoxidation reactions. The Journal of biological chemistry 271 , $9982-$ 9986 (1996).

Sell, D. R. \& M onnier, V. M. Structure elucidation of a senescence cross-link from human extracellular matrix. Implication of pentoses in the aging process. J Biol Chem 264, 21597-21602 (1989). Poulsen, M. W. et al. Advanced glycation endproducts in food and their effects on health. Food Chem Toxicol 60, 10-37, doi:10.1016/j.fct.2013.06.052 (2013).

Nicholl, I. D. et al. Increased levels of advanced glycation endproducts in the lenses and blood vessels of cigarette smokers. Mol M ed 4, 594-601 (1998).

Wellner, A., Huettl, C. \& Henle, T. Formation of M aillard reaction products during heat treatment of carrots. Journal of agricultural and food chemistry 59, 7992-7998, doi:10.1021/jf2013293 (2011). Scheijen, J. L. et al. Analysis of advanced glycation endproducts in selected food items by ultraperformance liquid chromatography tandem mass spectrometry: Presentation of a dietary AGE database. Food Chem 190, 1145-1150, doi:10.1016/j.foodchem.2015.06.049 (2016). Cordain, L. et al. Origins and evolution of the Western diet: health implications for the 21st century. Am J Clin Nutr 81, 341-354 (2005).

54 Uribarri, J. et al. Dietary advanced glycation end products and their role in health and disease. Advances in nutrition 6, 461-473, doi:10.3945/an.115.008433 (2015). 
Stirban, A., Gawlowski, T. \& Roden, M. Vascular effects of advanced glycation endproducts: Clinical effects and molecular mechanisms. Molecular metabolism 3, 94-108, doi:10.1016/j.molmet.2013.11.006 (2014).

Basta, G., Schmidt, A. M. \& De Caterina, R. Advanced glycation end products and vascular inflammation: implications for accelerated atherosclerosis in diabetes. Cardiovasc Res 63, 582-592 (2004).

Goldin, A., Beckman, J. A., Schmidt, A. M . \& Creager, M. A. Advanced glycation end products: sparking the development of diabetic vascular injury. Circulation 114, 597-605 (2006).

Jakus, V. \& Rietbrock, N. Advanced glycation end-products and the progress of diabetic vascular complications. Physiol Res 53, 131-142 (2004).

$\mathrm{Nin}, \mathrm{J}$. W. et al. Higher plasma levels of advanced glycation end products are associated with incident cardiovascular disease and all-cause mortality in type 1 diabetes: a 12-year follow-up study. Diabetes Care 34, 442-447 (2011).

Bierhaus, A. et al. Diabetes-associated sustained activation of the transcription factor nuclear factorkappaB. Diabetes 50, 2792-2808 (2001).

61 Brownlee, M. The pathobiology of diabetic complications: a unifying mechanism. Diabetes 54, 16151625 (2005). Monnier, V. M., Kohn, R. R. \& Cerami, A. Accelerated age-related browning of human collagen in diabetes mellitus. Proc Natl Acad Sci U SA 81, 583-587 (1984). Meerwaldt, R. et al. Simple non-invasive assessment of advanced glycation endproduct accumulation. Diabetologia 47, 1324-1330, doi:10.1007/s00125-004-1451-2 (2004).

Meerwaldt, R. et al. Skin autofluorescence, a measure of cumulative metabolic stress and advanced glycation end products, predicts mortality in hemodialysis patients. J Am Soc Nephrol 16, 3687-3693, doi:10.1681/asn.2005020144 (2005).

Noordzij, M. J. et al. Skin autofluorescence is increased in patients with carotid artery stenosis and peripheral artery disease. The international journal of cardiovascular imaging 28, 431-438, doi:10.1007/s10554-011-9805-6 (2012).

Osawa, S. et al. Skin Autofluorescence is Associated with Early-stage Atherosclerosis in Patients with Type 1 Diabetes. Journal of atherosclerosis and thrombosis, doi:10.5551/jat.35592 (2016). van Eupen, M. G. et al. Skin Autofluorescence and Pentosidine Are Associated With Aortic Stiffening: The M aastricht Study. Hypertension 68, 956-963, doi:10.1161/hypertensionaha.116.07446 (2016). Na, R., Stender, I. M., Ma, L. \& Wulf, H. C. Autofluorescence spectrum of skin: component bands and body site variations. Skin research and technology : official journal of International Society for Bioengineering and the Skin (ISBS) [and] International Society for Digital Imaging of Skin (ISDIS) [and] International Society for Skin Imaging (ISSI) 6, 112-117 (2000).

Buxton, T. \& Guilbault, G. G. Fluorometric analysis for N'-formylkynurenine in plasma and urine. Clin Chem 20, 765-768 (1974).

Malencik, D. A. \& Anderson, S. R. Dityrosine as a product of oxidative stress and fluorescent probe. Amino acids 25, 233-247, doi:10.1007/s00726-003-0014-z (2003). nicotinamide adenine dinucleotide fluorescence images of blood-perfused rat heart. Biophysical journal 72, 1849-1860, doi:10.1016/s0006-3495(97)78831-3 (1997).

Meerwaldt, R. et al. Skin autofluorescence is a strong predictor of cardiac mortality in diabetes. Diabetes Care 30, 107-112, doi:10.2337/dc06-1391 (2007). 
Nakayama, H. et al. Production and characterization of antibodies to advanced glycation products on proteins. Biochem Biophys Res Commun 162, 740-745 (1989).

Makita, Z., Vlassara, H., Cerami, A. \& Bucala, R. Immunochemical detection of advanced glycosylation end products in vivo. J Biol Chem 267, 5133-5138 (1992).

Horiuchi, S., Araki, N. \& Morino, Y. Immunochemical approach to characterize advanced glycation end products of the Maillard reaction. Evidence for the presence of a common structure. J Biol Chem 266, 7329-7332 (1991).

Ikeda, K. et al. N (epsilon)-(carboxymethyl)|ysine protein adduct is a major immunological epitope in proteins modified with advanced glycation end products of the Maillard reaction. Biochemistry $\mathbf{3 5}$, 8075-8083, doi:10.1021/bi9530550 (1996).

77 Reddy, S., Bichler, J., Wells-Knecht, K. J., Thorpe, S. R. \& Baynes, J. W. N epsilon-(carboxymethyl)lysine is a dominant advanced glycation end product (AGE) antigen in tissue proteins. Biochemistry $\mathbf{3 4}$, 10872-10878 (1995).

Nagai, R. et al. Hydroxyl radical mediates $\mathrm{N}$ epsilon-(carboxymethyl)|ysine formation from Amadori product. Biochem Biophys Res Commun 234, 167-172 (1997).

79 Berg, T. J. et al. The advanced glycation end product Nepsilon-(carboxymethyl)lysine is increased in serum from children and adolescents with type 1 diabetes. Diabetes Care 21, 1997-2002 (1998).

Schleicher, E. D., Wagner, E. \& Nerlich, A. G. Increased accumulation of the glycoxidation product $\mathrm{N}$ (epsilon)-(carboxymethyl)lysine in human tissues in diabetes and aging. J Clin Invest 99, 457-468, doi:10.1172/JCl119180 (1997).

81 Koito, W., Araki, T., Horiuchi, S. \& Nagai, R. Conventional antibody against Nepsilon(carboxymethyl)lysine (CML) shows cross-reaction to Nepsilon-(carboxyethyl)lysine (CEL): immunochemical quantification of CML with a specific antibody. J Biochem 136, 831-837, doi:10.1093/jb/mvh193 (2004).

82 Nagai, R. et al. Usefulness of antibodies for evaluating the biological significance of AGEs. Ann N Y Acad Sci 1126, 38-41, doi:10.1196/annals.1433.001 (2008).

83 Xiao, Y. \& Isaacs, S. N. Enzyme-linked immunosorbent assay (ELISA) and blocking with bovine serum albumin (BSA)--not all BSAs are alike. J Immunol Methods 384, 148-151, doi:10.1016/j.jim.2012.06.009 (2012).

$84 \mathrm{Li}$, L. et al. Formation and inhibition of Nepsilon-(carboxymethyl)lysine in saccharide-lysine model systems during microwave heating. Molecules (Basel, Switzerland) 17, 12758-12770, doi:10.3390/molecules171112758 (2012).

85 Smith, P. R. \& Thornalley, P. J. Influence of $\mathrm{pH}$ and phosphate ions on the kinetics of enolisation and degradation of fructosamines. Studies with the model fructosamine, $\mathrm{N}$ epsilon-1-deoxy-D-fructos-1yl-hippuryl-lysine. Biochem Int 28, 429-439 (1992).

Charissou, A., Ait-Ameur, L. \& Birlouez-Aragon, I. Evaluation of a gas chromatography/mass spectrometry method for the quantification of carboxymethyllysine in food samples. J Chromatogr A 1140, 189-194, doi:10.1016/j.chroma.2006.11.066 (2007).

Tareke, E., Forslund, A., Lindh, C. H., Fahlgren, C. \& Ostman, E. Isotope dilution ESI-LC-M S/MS for quantification of free and total Nepsilon-(1-Carboxymethyl)-L-Lysine and free Nepsilon-(1Carboxyethyl)-L-Lysine: comparison of total Nepsilon-(1-Carboxymethyl)-L-Lysine levels measured with new method to ELISA assay in gruel samples. Food Chem 141, 4253-4259, doi:10.1016/j.foodchem.2013.07.003 (2013). 
Delatour, T. et al. Analysis of advanced glycation endproducts in dairy products by isotope dilution liquid chromatography-electrospray tandem mass spectrometry. The particular case of carboxymethyllysine. J Chromatogr A 1216, 2371-2381 (2009).

Uribarri, J. et al. Advanced glycation end products in foods and a practical guide to their reduction in the diet. Journal of the American Dietetic Association 110, 911-916 e912, doi:10.1016/j.jada.2010.03.018 (2010).

Hull, G. L. J., Woodside, J. V., Ames, J. M . \& Cuskelly, G. J. Nع-(carboxymethyl)lysine content of foods commonly consumed in a Western style diet. Food Chemistry 131, 170-174 (2012).

Takedo, A. et al. Immunohistochemical study of advanced glycation end products in aging and Alzheimer's disease brain. Neurosci Lett 221, 17-20 (1996).

Sakata, N. et al. Increased advanced glycation end products in atherosclerotic lesions of patients with end-stage renal disease. Atherosclerosis 142, 67-77 (1999).

Shapiro, B. P. et al. Advanced glycation end products accumulate in vascular smooth muscle and modify vascular but not ventricular properties in elderly hypertensive canines. Circulation 118, 10021010, doi:10.1161/circulationaha.108.777326 (2008).

Ettre, L. S. The centenary of the invention of chromatography. Journal of chromatographic science $\mathbf{4 1}$, 225-226, doi:NO_DOI (2003).

Ettre, L. S. Chromatography of natural substances: an introduction. Journal of chromatographic science 46, 90-92 (2008).

Ettre, L. S. S., K.I. M. S. Tswett and the discovery of chromatography I: Early work (1899-1903). Chromatographia 35, 223-231 (1993).

Martin, A. J. \& Synge, R. L. Separation of the higher monoamino-acids by counter-current liquid-liquid extraction: the amino-acid composition of wool. The Biochemical journal 35, 91-121 (1941).

Snyder, L. R. K., Joseph J.; Dolan, John W. Introduction to Modern Liquid Chromatography. Third Edition edn, (John Wiley \& Sons, Inc., 2010).

101 Horvath, C. G. \& Lipsky, S. R. Use of liquid ion exchange chromatography for the separation of organic compounds. Nature 211, 748-749 (1966).

102 Swartz, M. E. UPLC: An Introduction and Review. Journal of Liquid Chromatography \& Related Technologies 28, 1253-1263 (2005).

103 Deemter van, J. J. Z., F.J.; Klinkenberg, A. Longitudinal diffusion and resistance to mass transfer as causes of nonideality in chromatography. Chemical Engineering Science 5, $271-289$ (1956).

104 Churchwell, M. I., Twaddle, N. C., M eeker, L. R. \& Doerge, D. R. Improving LC-MS sensitivity through increases in chromatographic performance: comparisons of UPLC-ES/MS/MS to HPLC-ES/MS/MS. J Chromatogr B Analyt Technol Biomed Life Sci 825, 134-143, doi:10.1016/j.jchromb.2005.05.037 (2005).

105 Griffiths, I. W. J. J. Thomson - the Centenary of His Discovery of the Electron and of His Invention of Mass Spectrometry. Rapid Commun. M ass Spectrom. 11, 2-16 (1997).

106 Paul, W. \& Steinwedel, H. Ein neues Massenspektrometer ohne Magnetfeld. Zeitschrift für Naturforschung 8, 448-450 (1953).

107 Dole, M. et al. M olecular Beams of Macroions. Journal of Chemical Physics 49, 2240-2249 (1968).

108 Fenn, J. B., Mann, M., M eng, C. K., Wong, S. F. \& Whitehouse, C. M. Electrospray ionization for mass spectrometry of large biomolecules. Science 246, 64-71 (1989). 
109

110

M cLafferty, F. W. Tandem M ass Spectrometry. Biological Mass Spectrometry 11, 493 (1983).

Teerlink, T., Barto, R., Ten Brink, H. J. \& Schalkwijk, C. G. Measurement of Nepsilon(carboxymethyl)lysine and Nepsilon-(carboxyethyl)lysine in human plasma protein by stable-isotopedilution tandem mass spectrometry. Clin Chem 50, 1222-1228 (2004).

Ames, J. M. Determination of $\mathrm{N}$ epsilon-(carboxymethyl)lysine in foods and related systems. Ann N Y Acad Sci 1126, 20-24 (2008).

Knecht, K. J. et al. Effect of diabetes and aging on carboxymethyllysine levels in human urine. Diabetes 40, 190-196 (1991).

Thornalley, P. J. et al. Quantitative screening of advanced glycation endproducts in cellular and extracellular proteins by tandem mass spectrometry. Biochem J 375, 581-592 (2003).

Schmitz, I., Zahn, H., Klostermeyer, H., Rabbel, K. \& Watanabe, K. [On the occurence of isopeptide bonds in heated milk protein (author's transl)]. Z Lebensm Unters Forsch 160, $377-381$ (1976).

Henle, T., Walter, H. \& Klostermeyer, H. Evaluation of the extent of the early M aillard-reaction in milk products by direct measurement of the Amadori-product lactuloselysine. Z Lebensm Unters Forsch 193, 119-122 (1991).

Thornalley, P. J. Measurement of protein glycation, glycated peptides, and glycation free adducts. Perit Dial Int 25, 522-533 (2005).

Ames, J. M. Mass spectrometry to detect the site specificity of advanced glycation/lipoxidation endproduct formation on protein: some challenges and solutions. Biochem Soc Trans 36, 1051-1054, doi:10.1042/bst0361051 (2008).

Anderson, M. M. \& Heinecke, J. W. Production of N(epsilon)-(carboxymethyl)lysine is impaired in mice deficient in NADPH oxidase: a role for phagocyte-derived oxidants in the formation of advanced glycation end products during inflammation. Diabetes 52, 2137-2143 (2003).

Srey, C. et al. Immunochemical and mass spectrometric analysis of Nepsilon-(carboxymethyl)lysine content of AGE-BSA systems prepared with and without selected antiglycation agents. Journal of agricultural and food chemistry 58, 11955-11961, doi:10.1021/jf102543g (2010).

Furey, A., Moriarty, M., Bane, V., Kinsella, B. \& Lehane, M. Ion suppression; a critical review on causes, evaluation, prevention and applications. Talanta 115, 104-122, doi:10.1016/j.talanta.2013.03.048 (2013).

Annesley, T. M. Ion suppression in mass spectrometry. Clin Chem 49, 1041-1044 (2003).

Dietzen, D. J. et al. Rapid comprehensive amino acid analysis by liquid chromatography/tandem mass spectrometry: comparison to cation exchange with post-column ninhydrin detection. Rapid Commun Mass Spectrom 22, 3481-3488, doi:10.1002/rcm.3754 (2008).

23 Struys, E. A., Jansen, E. E., Verhoeven, N. M. \& Jakobs, C. Measurement of urinary D- and L-2hydroxyglutarate enantiomers by stable-isotope-dilution liquid chromatography-tandem mass spectrometry after derivatization with diacetyl-L-tartaric anhydride. Clin Chem 50, 1391-1395 (2004). Nakayama, K. et al. Plasma alpha-oxoaldehyde levels in diabetic and nondiabetic chronic kidney disease patients. Am J Nephrol 28, 871-878 (2008). 


\section{MEASUREMENT OF}

\section{IN HUMAN PLASMA PROIIEN BY A}

single-column High-Performance Liquid Chromatography method WITH FLUORESCENCE DEIECTION 


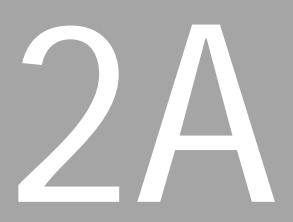

Measurement of pentosidine in human plasma protein by a single-column High-Performance Liquid Chromatography method with fluorescence detection

Jean LJ.M. Scheijen, Marjo P.H. van de Waarenburg, Coen D. A. Stehouwer and Casper G. Schalkwijk

Journal of Chromatography B 877; 610-614 (2009) 


\section{Abstract}

A rapid and sensitive single-column High-Performance Liquid Chromatography method and application for the detection of protein bound pentosidine is described. Pentosidine, a cross-link between arginine and lysine, is a well-characterized advanced glycation endproduct. In order to detect protein-bound pentosidine, plasma proteins were hydrolysed in $6 \mathrm{~N} \mathrm{HCL}$. Detection of pentosidine is done based on its own fluorescence characteristics using fluorimetric detection ( $E x=325 \mathrm{~nm}, E m=385 \mathrm{~nm}$ ). Separation is done, with a run-to-run time of 30 minutes, on a C18 Allspehere ODS-II column with a citric acid acetonitril buffer. This detection enables sensitive and specific determination of protein bound pentosidine in plasma with a detection limit of $2.2 \mathrm{nmol} / \mathrm{L}$ or $0.02 \mathrm{pmol} / \mathrm{mg}$ protein (signal-to-noise: 6). The intra-assay coefficient variation is $6.5 \%$ at a plasma pentosidine concentration of $0.47 \mathrm{pmol} / \mathrm{mg}$ protein and $2.0 \%$ at a concentration of $1.27 \mathrm{pmol} / \mathrm{mg}$ protein. The inter-assay coefficient variation is $3.1 \%$ at a plasma pentosidine concentration of $0.43 \mathrm{pmol} / \mathrm{mg}$ protein and $1.6 \%$ at a concentration of $1.40 \mathrm{pmol} / \mathrm{mg}$ protein. Linearity is tested in 4 different plasma samples and showed linearity $(0-200 \mathrm{nmol} / \mathrm{L}, \mathrm{r} 2>0.99)$. Recovery of pentosidine in 4 different plasma samples at different concentration levels is $102 \pm 10 \%$ (mean \pm SD). Using this method protein bound pentosidine concentration is investigated in healthy controls ( $n=24$, age $67 \pm 9$ years) and patients with end stage renal disease $(n=24$, age $65 \pm 10$ years). Higher plasma concentrations of protein bound pentosidine are measured in the patient group as compared with the control group $3.05(2.03-3.92) \mathrm{pmol} / \mathrm{mg}$ protein and 0.21 (0.19-0.33) $\mathrm{pmol} / \mathrm{mg}$ protein, respectively (median (interquartile range), $\mathrm{p} \varangle 0.00001$ ). These results are consistent with previously reported results. 


\section{Introduction}

Glycation is the nonenzymatic reaction of glucose or other reducing sugars with amino groups of proteins. The amino groups of the side chains of arginine and lysine are the primary targets for this type of posttranslational modification. Over time, the initial glycation products may undergo intramolecular rearrangements and oxidation reactions and ultimately transform into stable, so-called advanced glycation endproducts (AGEs). AGEs represent a mixture of different products such as pentosidine and $\mathrm{N}^{\varepsilon}$-(carboxymethyl)|ysine (CML). AGEs have different biological functions: some are protein cross-links such as pentosidine and some, such as CML, are recognition factors for specific AGE-binding receptors. The physiological consequences of AGEs in ageing and in the aetiology of diabetic complications ${ }^{1}$ and in the development of age-related diseases such as inflammation ${ }^{2}$, atherosclerosis ${ }^{3}$ and neurodegenerative disorders ${ }^{4}$ has been described.

Despite progress in this field, a more comprehensive understanding of the putative effects of AGEs in the pathophysiology of these diseases is needed. In addition, the measurement of AGEs may also help to identify patients with a high risk for poor outcome and may thus help in risk stratification ${ }^{5,6}$. However, progress in the field is hampered by the lack of an easy method for the detection of AGEs in the laboratory. We need a rapid, simple and reliable method for the detection of AGEs.

AGEs have traditionally been detected by enzyme-linked immunosorbent assay (ELISA) $)^{7-9}$. For several reasons the use of antisera for quantitative immunoassays of protein-bound AGEs is questionable. Reproducibility and sensitivity of such an assay are not optimal, because the specificity of the antibodies is often difficult to define and, because of steric constraints, not all AGE epitopes on the protein may be available for interaction with the antibody. Thus, AGE measurements with immunoassays should be interpreted with care. A better approach for the quantitative determination of specific AGEs in proteins is the use of a specific analytical technique. For the determination of AGE levels in both tissue and blood samples, high performance liquid chromatography (HPLC) measurements ${ }^{10-16}$ and several mass spectrometry methods have been developed including gas chromatography mass spectrometry (GC-MS) ${ }^{17}$, and liquid chromatography tandem mass spectrometry (LC-M SM S) ${ }^{18,19}$. Although the latter is considered to be the most accurate technique available at this moment for the detection of AGEs, a disadvantage is that this method is very expensive and not suitable for routine clinical use.

Pentosidine, one of the best-characterized AGEs, is a fluorescent cross-link between arginine and lysine. Because pentosidine is stable under the conditions used for acid protein hydrolysis and can be detected at very low concentrations based upon its fluorescence properties, pentosidine can be regarded as a biomarker for AGEs. Until now, pentosidine has been quantified by ELISA ${ }^{7,9}$, by HPLC ${ }^{10-16,20-25}$ and by LC-M SM ${ }^{19,28}$. In addition to the restrictions to use immunoassays for the detection of AGES as described above, antibodies against pentosidine or specifc ELISA systems are not commercially available. 
Accurate quantification of pentosidine by HPLC could only be obtained with cumbersome double-chromagraphic systems ${ }^{10,13,14,20}$, with ion-exchange HPLC with long run-times and high variations in retention-time ${ }^{21}$, with RP-HPLC with the use of ion-pairing agent with low recoveries ${ }^{10,12,26}$ or HPLC analysis with elaborate solid phase extraction (SPE) sample preparation $^{16}$.

We now describe a rapid, simple and sensitive one-column reversed-phase HPLC method for the detection of pentosidine in plasma protein hydrolysates.

\section{Materials and methods}

\section{Instrumentation}

Samples were analysed by reversed phase HPLC-fluorescence using an Allsphere ODS-2 (150x4.6 mm, $3 \mu \mathrm{m})$ analytical column fitted with an Allsphere ODS-2 (7.5x4.6, $5 \mu \mathrm{m}$ ) precolumn (Alltech/Grace, Breda, The Netherlands). Detection was carried out using a Jasco type 821-FP spectrofluorometer (Jasco Benelux, M aarssen, The Netherlands) set at an excitation and emission wavelength of 325 and $385 \mathrm{~nm}$, respectively. HPLC analysis was performed using a binary high pressure gradient at a flow of $1 \mathrm{~mL} / \mathrm{min}$ using two Model PU-980 pumps (Jasco Benelux, Maarssen, The Netherlands). Solvent A was $25 \mathrm{mmol} / \mathrm{L}$ citric acid and solvent B was (50/50 v/v) ACN/25 mmol/L citric acid. A linear gradient was started at $99 \%$ solvent A which was changed within 15 minutes to $90 \%$ solvent A. After cleaning the column with $100 \%$ solvent B during 5 minutes the column was equilibrated for 8 minutes at the initial composition. Injection volume was $10 \mu \mathrm{L}$ and column temperature was set at $20^{\circ} \mathrm{C}$ using a Spark Mistral column oven (Spark Holland B.V., Emmen, The Netherlands). Samples where thermostatted at $6^{\circ} \mathrm{C}$ using an autoinjector model 717 Plus Autosampler (Waters, Etten Leur, The Netherlands). Chromatograms were acquired and processed with Totalchrom (Perkin-Elmer, version 6.2.0.0.0:B27, Zoetermeer, The Netherlands).

\section{M aterials}

HPLC-grade acetonitril (ACN) was obtained from Chromanorm (Prolabo, Paris, France). Citric acid (GR for analysis), sodium hydroxide and hydrochloric acid $(\mathrm{HCl})$ fuming $(37 \%)$ were obtained from M erck (Darmstadt, Germany). Trichloroacetic acid, boric acid $(99.5 \%)$ and trifluoroacetic acid (TFA) (99+\%) were obtained from Sigma (SigmaUltra min. 99,0\%) (Zwijndrecht, The Netherlands). Ultra pure waters was generated by a Super-Q system (Millipore, Amsterdam, The Netherlands) and sodium borohydride was obtained from Fluka (Buchs, Schweiz). Pentosidine standard was obtained from IMARS (International Maillard Reaction Society, http://imars.case.edu/). Levels determined with this standard are four times lower than published by Sell et al. ${ }^{27}$ and agree with Dyer et al. ${ }^{28}$. 


\section{Plasma samples}

Heparinized plasma samples were obtained form 24 healthy volunteers (16 male, 8 female, mean age $67 \pm 9$ years) and 24 uremic patients on peritoneal dialysis (PD) (14 male, 10 female, mean age $65 \pm 10$ years).

\section{Sample preparation}

In a $10 \mathrm{~mL}$ glass tube with a Teflon-lined screw-cap $50 \mu \mathrm{L}$ plasma was mixed with $100 \mu \mathrm{L}$ water. To prevent a potential artifactual formation of pentosidine from early glycation products during sample preparation, plasma samples were reduced by $500 \mu \mathrm{L}$ sodium borohydride borate buffer $(200 \mathrm{mmol} / \mathrm{L}, \mathrm{pH}=9.2)$ before precipitation. This mixture was allowed to stand for 2 hours at room temperature. Proteins were then precipitated by addition of $2 \mathrm{~mL} 20 \%$ trichloroacetic acid and centrifuged for 10 minutes $\left(4^{\circ} \mathrm{C}\right)$ at $4500 \mathrm{~g}$. The supernatant was carefully removed by aspiration with a Pasteur pipette. The protein pellet was washed once by adding $2 \mathrm{~mL} 5 \%$ trichloroacetic acid followed by centrifugation and removal of the supernatant as described above (The effect of this extra washing step on absolute peak area and recovery was tested negative). For recovery and linearity experiments $50 \mu \mathrm{L}$ of pentosidine standard (0-200 nmol/L) was added. Samples were hydrolyzed by adding $50 \mu \mathrm{L} 6 \mathrm{~N} \mathrm{HCl}$ and incubation for 18 hours at $110^{\circ} \mathrm{C}$. After hydrolysis, samples were evaporated to dryness at $80^{\circ} \mathrm{C}$ under a stream of nitrogen gas and reconstituted in $200 \mu \mathrm{L} 25 \mathrm{mmol} / \mathrm{L}$ citric acid/L. This solution was centrifuged for 15 minutes $\left(4^{\circ} \mathrm{C}\right)$ at $14000 \mathrm{rpm}$. Ten micro liter of this solution (equals $2.5 \mu \mathrm{L}$ plasma) was injected on the HPLC system.

\section{Standard pentosidine}

Stock standard pentosidine was prepared by dissolving pentosidine in $0.1 \%(\mathrm{v} / \mathrm{v})$ TFA at a concentration of $20 \mu \mathrm{M}$. Standard was divided in portions and stored at $-20^{\circ} \mathrm{C}$ prior to use.

\section{Determination of protein in plasma}

Pentosidine concentration was expressed as $\mathrm{pmol} / \mathrm{mg}$ protein in plasma. Protein concentration in plasma was analysed using Bradford reagent (Biorad Laboratories GMBH, München, Germany) ${ }^{29}$. The calibration curve was established using bovine albumin fraction V (Sigma, Zwijndrecht, The Netherlands). 


\section{Statistical Analysis}

The method validation data were expressed as mean and SD. The patient study data were expressed as median and interquartile ranges (IQR). To detect group differences we applied the Mann-Whitney $\mathrm{U}$ test.

\section{Results}

\section{Reversed phase chromatography}

During method development we tested first cation-exchange chromatography, as described by M itsuru Saito et al. 21 and different ion-pair based HPLC systems. However, with these methods we observed retention time variations of $>1$ min with no baseline separation of pentosidine (data not shown). In contrast, with RP-HPLC, we found a retention time of $13.24 \pm 0.07$ min (CV $0.5 \%, n=50$ different plasma samples) and a baseline separated pentosidine peak. A chromatogram of a pentosidine standard, a typical chromatogram of a plasma sample from a healthy control and from a uremic patient on peritoneal dialysis are shown in figure 2A.1. The baseline separated pentosidine peak in plasma makes automatic integration possible.
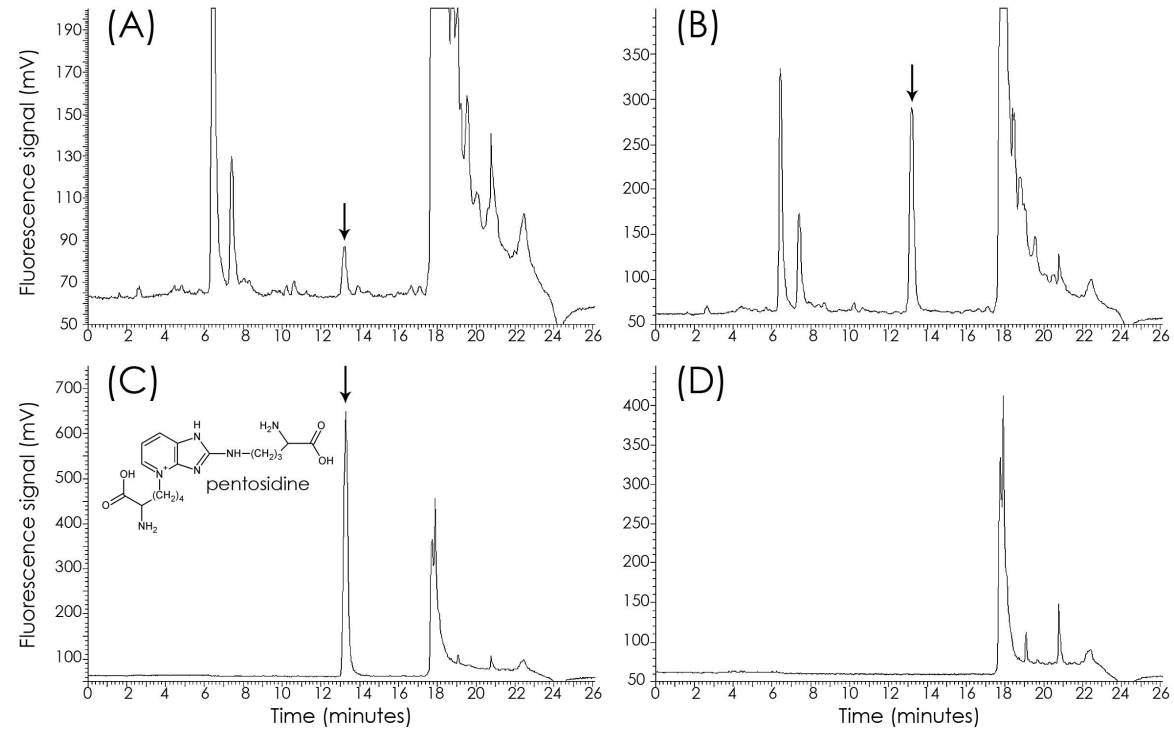

Figure 2A.1 Representative chromatograms of a standard solution of pentosidine (C), a plasma sample from a healthy control (A), a uremic patient on peritoneal dialysis (B) and a blank sample (D). The elution of pentosidine is indicated with an arrow mark with a peak area (and concentration) of pentosidine in $A=314,197(0.60$ $\mathrm{pmol} / \mathrm{mg}$ protein), in $B=3,638,638$ (6.06 pmol/mg protein), in $C=8,901,011$ (460 nM) and in $D=0$ (0 pmol). Pentosidine is clearly baseline separated which makes time consuming manual integration afterwards not necessary. 


\section{Stability of pentosidine}

To make large number of measurements within one run possible, we tested the stability of pentosidine under different conditions. The stability of pentosidine was tested in solvents with neutral $\mathrm{pH}$, acidic $\mathrm{pH}(\mathrm{pH}=2.5)$ and basic $\mathrm{pH}(\mathrm{pH}=10.6)$. Pentosidine was at least stable for 35 hours in all the tested solvents when samples were stored in the autoinjector at $6^{\circ} \mathrm{C}$ (figure. 2A.2).

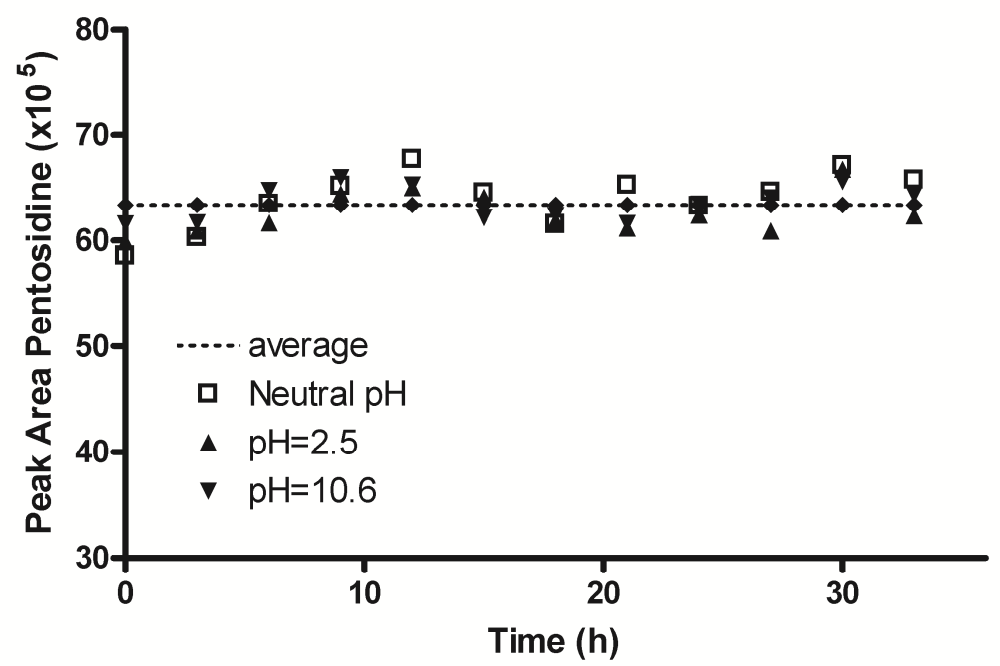

Figure 2A.2 The stability of pentosidine at different $\mathrm{pH}$. Pentosidine was diluted in citric acid buffer ( $\mathrm{pH}=2.5$ ), in citrate buffer $(\mathrm{pH}=10.6)$ and in water to a final concentration of $32 \mathrm{nmol} / \mathrm{L}$. Pentosidine concentration was measured upon a storage at $6^{\circ} \mathrm{C}$ for 35 hours.

\section{Linearity and lower limit of quantification}

Linearity of the detection of pentosidine was tested in water (with and without hydrolysis) and matrix by adding pentosidine standard during preparation of water and different plasma samples (table 2A.1 and figure 2A.3). The slope, tested in 4 different plasma samples and in water measured on two different days, was $19685 \pm 3.4 \%$ (mean $\pm \mathrm{CV} \%$ ). Acid hydrolysis had no effect on the peak areas of pentosidine, therefore calibration was carried out with non-hydrolysed standards. Also no matrix effect was observed as tested in different plasma samples (table 2A.1 and figure 2A.3). The limit of detection (signal-to-noise: 6) of pentosidine was $2.2 \mathrm{nmol} / \mathrm{L}$ or $0.02 \mathrm{pmol} / \mathrm{mg}$ protein, corresponding to a concentration of $0.02 \mathrm{pmol} / \mathrm{mg}$ protein. 
Table 2A.1 Calibration curves of pentosidine in different matrices

\begin{tabular}{lllll}
\hline Matrix & Slope & Y-intercept & $\mathbf{r}^{2}$ & Conc. Range (nmol/ L) \\
\hline Water (without hydrolysis) & 19359 & - & 0.9997 & $0-200$ \\
Water (with hydrolysis) & 18300 & - & 0.9991 & $0-200$ \\
Plasma A & 19571 & $483386(* 24.7)$ & 0.9988 & $0-200$ \\
Plasma B & 20082 & $550906(* 27.4)$ & 0.9984 & $0-200$ \\
Plasma C & 19849 & $1027646(* 51.8)$ & 0.9983 & $0-75$ \\
Plasma D & 20630 & $473838(* 23.0)$ & 0.9959 & $0-75$ \\
Mean & $\mathbf{1 9 7 3 1}$ & & & \\
CV(\%) & $\mathbf{3 . 6}$ & & & \\
\hline
\end{tabular}

* Endogenous pentosidine in plasma (nmol/L)

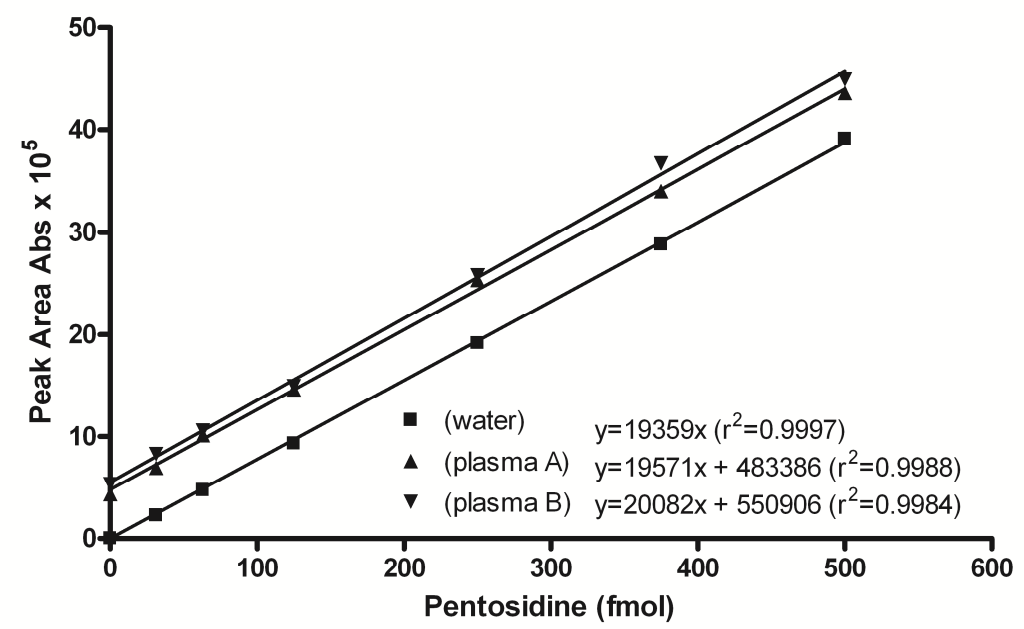

Figure 2A.3 Calibration curves of pentosidine in water and in plasma samples. Linearity of the detection of pentosidine was tested in water and in two different plasma samples by the addition of pentosidine standard ( 0 $200 \mathrm{nmol} / \mathrm{L}$ ) during sample preparation. No Matrix-effect was observed. Y-axis intercept in both plasma sample shows endogenous pentosidine (resp. $24.7 \mathrm{nmol} / \mathrm{L}$ in plasma $\mathrm{A}$ and $27.4 \mathrm{nmol} / \mathrm{L}$ in plasma B).

\section{Recovery and precision}

The intra-assay variation of the method was determined in two different plasma samples analysed in one batch during one day. The intra-assay variation was $6.5 \%$ as determined in a plasma sample $(n=9)$ with a mean concentration of $0.47 \mathrm{pmol} / \mathrm{mg}$ protein and $2.0 \%$ in a plasma sample $(n=10)$ with a mean concentration of $1.27 \mathrm{pmol} / \mathrm{mg}$ protein. The interassay variation of the method was determined in two different plasma samples divided into batches and analysed during different days. 
The inter-assay variation was $3.1 \%$ as determined in a plasma sample $(n=10)$ with a mean concentration of $0.43 \mathrm{pmol} / \mathrm{mg}$ protein and $1.6 \%$ in a plasma sample $(n=10)$ with a mean concentration of $1.40 \mathrm{pmol} / \mathrm{mg}$ protein. Recovery of pentosidine was $102+10 \%$ as determined in 4 different plasma samples (table 2A.2).

Table 2A.2. Recovery of pentosidine in 4 different plasma samples.

\begin{tabular}{|c|c|c|c|}
\hline Matrix & Added amount (fmol*) & Measured (fmol*) & Recovery (\%) \\
\hline \multirow[t]{6}{*}{ Plasma A } & 500 & 563 & 101 \\
\hline & 375 & 439 & 102 \\
\hline & 250 & 327 & 108 \\
\hline & 125 & 189 & 106 \\
\hline & 63 & 131 & 119 \\
\hline & 0 & 58 & - \\
\hline \multirow[t]{6}{*}{ Plasma B } & 500 & 580 & 102 \\
\hline & 375 & 474 & 108 \\
\hline & 250 & 333 & 106 \\
\hline & 125 & 193 & 99 \\
\hline & 63 & 136 & 108 \\
\hline & 0 & 70 & - \\
\hline \multirow[t]{5}{*}{ Plasma C } & 188 & 318 & 100 \\
\hline & 125 & 261 & 104 \\
\hline & 63 & 190 & 94 \\
\hline & 31 & 166 & 110 \\
\hline & 0 & 131 & - \\
\hline \multirow[t]{7}{*}{ Plasma D } & 188 & 262 & 104 \\
\hline & 125 & 186 & 96 \\
\hline & 63 & 123 & 91 \\
\hline & 31 & 89 & 71 \\
\hline & 0 & 67 & - \\
\hline & & Mean Recovery (\%) & 102 \\
\hline & & Recovery SD (\%) & 10 \\
\hline
\end{tabular}

(* per injected volume of $10 \mu \mathrm{L}$ )

\section{Comparison of pentosidine concentration between cases and controls}

We analyzed protein bound pentosidine in 24 healthy volunteers and in 24 uremic patients on peritoneal dialysis. The median (IQR) concentration of protein bound pentosidine was significantly higher in the peritoneal dialysis patients than in the healthy control group 3.05 (2.03-3.92) pmol/mg protein and $0.21(0.19-0.33) \mathrm{pmol} / \mathrm{mg}$ protein, respectively, $p \varangle 0.00001$ ) (figure $2 A .4$ ). 


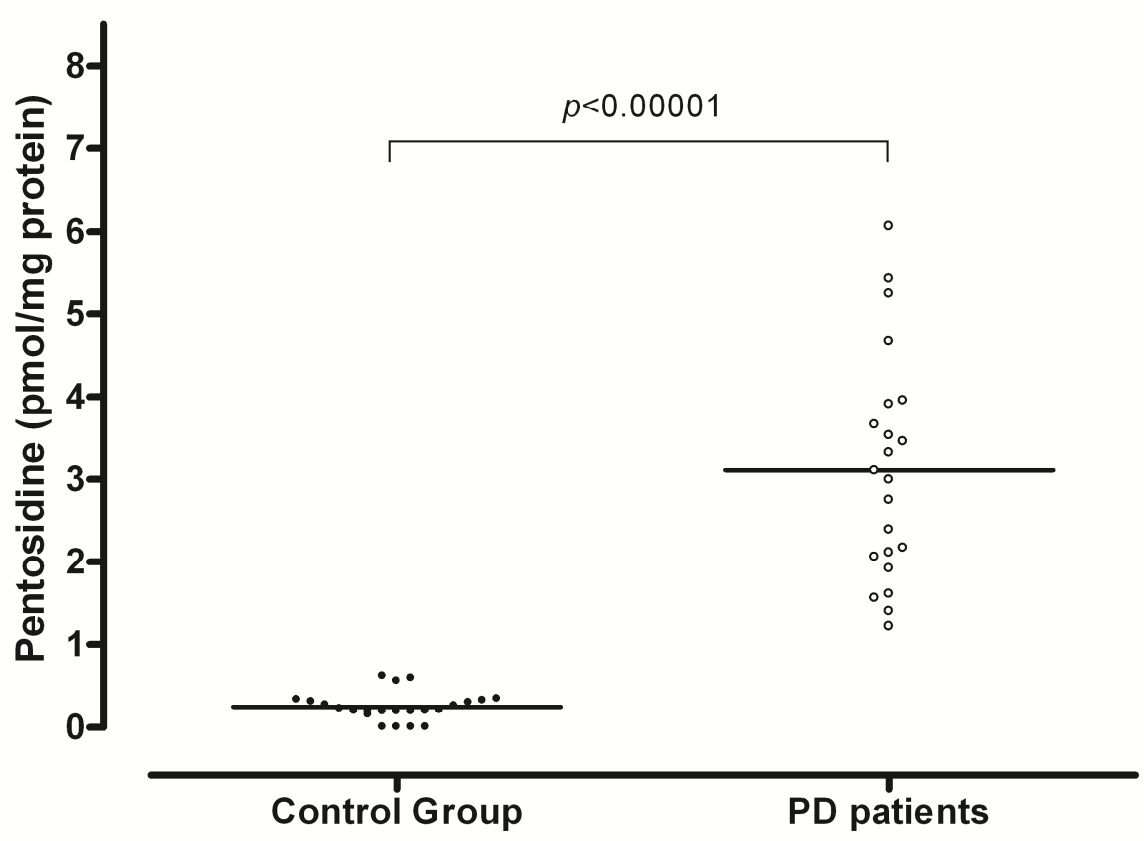

Figure 2A.4 Protein bound pentosidine concentration in plasma of healthy controls and in peritoneal dialysis (PD) patients. Data are presented as median (line) and separate datapoints.

\section{Discussion}

We describe here a rapid, simple and reliable method to determine protein bound pentosidine in plasma by a single step RP-based HPLC separation with fluorescent detection. The combination between a very simple sample preparation and a short runtime makes this method a strong and easy tool to determine protein bound pentosidine in a large number of plasma samples.

Many techniques have been used to quantify pentosidine ${ }^{10-14,19,21-23,30}$ with some disadvantages such as long run-times ${ }^{10,13,14,23}$, elaborate sample preparation ${ }^{16}$ or analysis on expensive LC-M S/MS equipment ${ }^{19,30}$.

Two main advantages of using "normal" reversed phase chromatography instead of the ion-pair based chromatography, which is commonly used in this field ${ }^{9,10,12-15,23,25}$, is a very clean chromatogram and a very stable retention-time. Indeed, we found very clean chromatographs, with baseline separation of pentosidine in plasma, which makes afterwards time-consuming manual integration unnecessary. With a retention time of only $13.24 \pm 0.07 \mathrm{~min}$ and a run-to-run time of approximately $30 \mathrm{~min}$, we can run, unattended, 48 samples a day. 
Another advantage of the described sample preparation is that it is also suitable for the simultaneous analysis of protein-bound pentosidine with HPLC-fluorescence and for protein bound AGEs such as $\mathrm{N} \varepsilon$-(carboxymethyl)lysine $(\mathrm{CML}$ ) and $\mathrm{N} \varepsilon$-(carboxyethyl)lysine (CEL) with LC-MS/MS technique ${ }^{18}$. Only $50 \mu \mathrm{L}$ of plasma is needed for both methods. After hydrolysis the samples can be split; one part for the HPLC-fluorescence analysis of pentosidine and one part for the LC-M S/MS analysis.

With this method we measured protein bound pentosidine in healthy volunteers and patients on peritoneal dialysis with a limit of detection of $0.02 \mathrm{pmol} / \mathrm{mg}$ protein, which is comparable with limit of detection as described in literature ${ }^{15,20,22}$. The mean plasma concentration of protein bound pentosidine in healthy controls as measured with the method described above was $0.21 \mathrm{pmol} / \mathrm{mg}$ protein. The absolute concentrations of protein bound pentosidine in healthy controls described in literature are divergent, ranging from 0.95 to $2.0 \mathrm{pmol} / \mathrm{mg}$ protein ${ }^{10,31-33}$ and our value of $0.21 \mathrm{pmol}$ pentosidine per mg protein is lower. Differences in the characteristics of the controls and a lack of an international pentosidine standard may explain the differences. Therefore, direct comparison of the absolute levels of pentosidine as measured in different studies is difficult. The 15-fold increase in plasma protein bound pentosidine in uremic patients in comparison to healthy controls is in agreement with previous results ${ }^{9,34,35-32}$.

The detection of AGEs is currently of much experimental and clinical interest, in particular because data of few epidemiological studies have demonstrated associations of plasma levels of AGEs with vascular complications ${ }^{5,6}$.

Although studies demonstrating a causal role of AGEs in the development of cardiovascular disease are limited, it might be that specific AGEs are risk factors of cardiovascular disease. In accordance with this, high serum levels of AGEs predict increased coronary heart disease mortality in non-diabetic women ${ }^{5}$ and a recent study demonstrated that serum pentosidine concentration is an independent prognostic factor for heart failure 6 . However, AGEs in these studies were measured with immunoassays and AGEs measurements with immunoassays should be interpreted with care.

In conclusion, we describe a fast, simple and reliable method for the quantitation of pentosidine. This method may help to obtain a better understanding of the putative effects of pentosidine in the pathophysiology of different diseases such as vascular complications and to test whether pentosidine measurements can be used to identify patients with a high risk for poor outcome and may thus help in risk stratification.

\section{Acknowledgements}

The authors would like to thank the Department of Surgery (Maastricht University, Maastricht, The Netherlands) for facilitating the chromatographic system and H.M.H. van Eijk PhD for his valuable comments. 


\section{References}

1

2

3

4

Goldin, A., Beckman, J. A., Schmidt, A. M \& \& Creager, M. A. Advanced glycation end products: sparking the development of diabetic vascular injury. Circulation 114, 597-605 (2006).

Yan, S. F., Ramasamy, R. \& Schmidt, A. M. Mechanisms of disease: advanced glycation end-products and their receptor in inflammation and diabetes complications. Nat Clin Pract Endocrinol Metab 4, 285-293 (2008).

3 Basta, G., Schmidt, A. M. \& De Caterina, R. Advanced glycation end products and vascular inflammation: implications for accelerated atherosclerosis in diabetes. Cardiovasc Res 63, 582-592 (2004).

Takeuchi, M. \& Yamagishi, S. Possible involvement of advanced glycation end-products (AGEs) in the pathogenesis of Alzheimer's disease. Curr Pharm Des 14, 973-978 (2008).

Kilhovd, B. K. et al. High serum levels of advanced glycation end products predict increased coronary heart disease mortality in nondiabetic women but not in nondiabetic men: a population-based 18year follow-up study. Arterioscler Thromb Vasc Biol 25, 815-820 (2005).

Koyama, Y. et al. High serum level of pentosidine, an advanced glycation end product (AGE), is a risk factor of patients with heart failure. J Card Fail 13, 199-206 (2007).

Sanaka, T. et al. Plasma pentosidine levels measured by a newly developed method using ELISA in patients with chronic renal failure. Nephron 91, 64-73 (2002).

Izuhara, Y. et al. A sensitive and specific ELISA for plasma pentosidine. Nephrol Dial Transplant 14, 576-580 (1999).

Taneda, S. \& M onnier, V. M. ELISA of pentosidine, an advanced glycation end product, in biological specimens. Clin Chem 40, 1766-1773 (1994).

Odetti, P., Fogarty, J., Sell, D. R. \& Monnier, V. M. Chromatographic quantitation of plasma and erythrocyte pentosidine in diabetic and uremic subjects. Diabetes 41, 153-159 (1992).

1 Mikulikova, K., Eckhardt, A., Kunes, J., Zicha, J. \& Miksik, I. Advanced glycation end-product pentosidine accumulates in various tissues of rats with high fructose intake. Physiol Res 57, 89-94 (2008).

Spacek, P. \& Adam, M. Pentosidine in osteoarthritis: HPLC determination in body fluids and in tissues. Rheumatol Int 26, 923-927 (2006).

Slowik-Zylka, D. et al. A sensitive and specific HPLC method for the determination of total pentosidine concentration in plasma. J Biochem Biophys M ethods 61, 313-329 (2004).

Wilker, S. C., Chellan, P., Arnold, B. M. \& Nagaraj, R. H. Chromatographic quantification of argpyrimidine, a methylglyoxal-derived product in tissue proteins: comparison with pentosidine. Anal Biochem 290, 353-358 (2001).

Takahashi, M., Hoshino, H., Kushida, K., Kawana, K. \& Inoue, T. Direct quantification of pentosidine in urine and serum by HPLC with column switching. Clin Chem 42, 1439-1444 (1996).

Floridi, A., Trizza, V., Paolotti, P. \& Lucarelli, C. Analytical strategy for the assessment of the protein glycation status in uremic patients by high-performance liquid chromatography. J Chromatogr A 846, 65-71 (1999).

Lapolla, A. et al. Glyoxal and methylglyoxal levels in diabetic patients: quantitative determination by a new GC/M S method. Clin Chem Lab M ed 41, 1166-1173 (2003). 
Teerlink, T., Barto, R., Ten Brink, H. J. \& Schalkwijk, C. G. Measurement of Nepsilon(carboxymethyl)lysine and Nepsilon-(carboxyethyl)lysine in human plasma protein by stable-isotopedilution tandem mass spectrometry. Clin Chem 50, 1222-1228 (2004).

Thornalley, P. J. et al. Quantitative screening of advanced glycation endproducts in cellular and extracellular proteins by tandem mass spectrometry. Biochem J 375, 581-592 (2003).

\section{Takahashi, M., Hoshino, H., Kushida, K. \& Inoue, T. Direct measurement of crosslinks, pyridinoline,} deoxypyridinoline, and pentosidine, in the hydrolysate of tissues using high-performance liquid chromatography. Anal Biochem 232, 158-162 (1995).

Saito, M., M arumo, K., Fujii, K. \& Ishioka, N. Single-column high-performance liquid chromatographicfluorescence detection of immature, mature, and senescent cross-links of collagen. Anal Biochem 253, 26-32 (1997).

Chen, J. R. et al. Direct detection of crosslinks of collagen and elastin in the hydrolysates of human yellow ligament using single-column high performance liquid chromatography. Anal Biochem 278, 99105 (2000).

Miyata, T. et al. Renal catabolism of advanced glycation end products: the fate of pentosidine. Kidney Int 53, 416-422 (1998).

Ahmed, N. \& Thornalley, P. J. Chromatographic assay of glycation adducts in human serum albumin glycated in vitro by derivatization with 6-aminoquinolyl-N-hydroxysuccinimidyl-carbamate and intrinsic fluorescence. Biochem J 364, 15-24 (2002).

Spacek, P. \& Adam, M. HPLC M ethod for pentosidine determination in urine, serum, and tissues as a marker of glycation and oxidation loading of the organism. J Liq Chrom Rel Technol 25, 1807-1820 (2002).

Agalou, S., Ahmed, N., Dawnay, A. \& Thornalley, P. J. Removal of advanced glycation end products in clinical renal failure by peritoneal dialysis and haemodialysis. Biochem Soc Trans 31, 1394-1396 (2003).

(1)
matrix. Implication of pentoses in the aging process. J Biol Chem 264, 21597-21602 (1989).

Dyer, D. G., Blackledge, J. A., Thorpe, S. R. \& Baynes, J. W. Formation of pentosidine during nonenzymatic browning of proteins by glucose. Identification of glucose and other carbohydrates as possible precursors of pentosidine in vivo. J Biol Chem 266, 11654-11660 (1991).

Bradford, M. M. A rapid and sensitive method for the quantitation of microgram quantities of protein utilizing the principle of protein-dye binding. Anal Biochem 72, 248-254 (1976).

Ahmed, N. \& Thornalley, P. J. Quantitative screening of protein biomarkers of early glycation, advanced glycation, oxidation and nitrosation in cellular and extracellular proteins by tandem mass spectrometry multiple reaction monitoring. Biochem Soc Trans 31, 1417-1422 (2003).

1 Friedlander, M. A., Wu, Y. C., Elgawish, A. \& Monnier, V. M. Early and advanced glycosylation end products. Kinetics of formation and clearance in peritoneal dialysis. J Clin Invest 97, 728-735 (1996).

2 Odetti, P. et al. Correlation between pentosidine and endothelin-1 in subjects undergoing chronic hemodialysis. Horm M etab Res 38, 817-820 (2006).

3 Stein, $G$. et al. Influence of dialysis modalities on serum AGE levels in end-stage renal disease patients. Nephrol Dial Transplant 16, 999-1008 (2001).

Lapolla, A. et al. Evaluation of glyoxal and methylglyoxal levels in uremic patients under peritoneal dialysis. Ann N Y Acad Sci 1043, 217-224 (2005).

5 Suliman, M. E. et al. Plasma pentosidine is associated with inflammation and malnutrition in endstage renal disease patients starting on dialysis therapy. J Am Soc Nephrol 14, 1614-1622 (2003). 


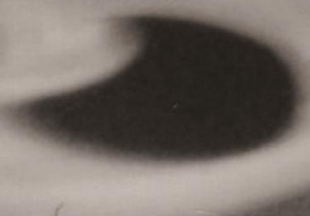

MEASUREMENT OF

advanced glycation endproducts

IN HUMAN PLASMA BY ultra-performance liquid chromatography tandem mass spectrometry 


\section{$2 B$}

Measurement of advanced glycation endproducts in human plasma by ultra-performance liquid chromatography tandem mass spectrometry*

Nordin M. J. Hanssen\#, Lian Engelen", Isabel Ferreira, Jean L J. M. Scheijen, Maya S. Huijberts, M arleen M. J. van Greevenbroek, Carla J. H. van der Kallen, Jacqueline M. Dekker, Giel Nijpels, Coen D. A. Stehouwer, and Casper G. Schalkwijk

${ }^{*}$ Equal contribution

J Clin Endocrinol M etab. 2013 Aug; 98(8): E1369-73* 


\section{Abstract}

Experimental and histological data suggest a role for advanced glycation endproducts (AGEs) in diabetic complications and in the development of age-related diseases such as inflammation, atherosclerosis and neurodegenerative disorders. To investigate these diseases in more detail a highly sensitive, selective and rapid ultra-performance liquid chromatography tandem mass spectrometry (UPLC-M S/MS) method for the simultaneous determination of $\mathrm{N}^{\varepsilon}$-(carboxymethyl)lysine (CML), $\mathrm{N}^{\varepsilon}$-(carboxyethyl)lysine (CEL) and $\mathrm{N}^{\delta}$-(5-hydro-5-methyl-4-imidazolon-2-yl)-ornithine (M G-H1) in plasma is described. The nonenzymatic reaction of glucose or other reducing sugars with amino groups of the side chains of arginine and lysine in proteins leads to the formation of these AGEs, including CML, CEL and M G-H1.

Protein-bound and free plasma AGEs were determined using a butanolic derivatisation in protein hydrolysate or the supernatant of deproteinized plasma, respectively. Derivatized CML, CEL and MG-H1 were separated on a reversed-phase UPLC C18 column and detected in ESI positive multiple reaction monitoring (M RM).

Calibration curves were linear throughout selected ranges. Responsefactors for CML, CEL and M G-H1 ranged between 3.1 and $13.5 \mathrm{CV}(\%)$. Intra- and inter-assay CVs, in proteinbound and free plasma AGEs, were between $2.8 \%$ and $23.6 \%$. With the use of the developed method, increased protein-bound and free plasma levels of CML, CEL and MG-H1 were found in uremic patients as compared to healthy controls.

In conclusion, the described method proofed to be suitable in studying AGEs and help us to understand the putative effects of AGEs in the pathophysiology of different diseases. 


\section{Introduction}

Glycation is the nonenzymatic reaction of glucose or other reducing sugars with amino groups of proteins. The amino groups of the side chains of arginine and lysine are the primary targets for this type of posttranslational modification. Over time, the initial glycation products may undergo intramolecular rearrangements and oxidation reactions and ultimately transform into stable, so-called advanced glycation endproducts (AGEs). AGEs represent a mixture of different products such as pentosidine, $\mathrm{N}^{\varepsilon}$-(carboxymethyl)|ysine (CM L), $\mathrm{N}^{\varepsilon}$-(carboxyethyl)lysine (CEL) and $\mathrm{N}^{\delta}$-(5-hydro-5-methyl4-imidazolon-2-yl)-ornithine (MG-H1). AGEs have different biological functions: some are protein cross-links such as pentosidine and some, such as $\mathrm{CML}$, are recognition factors for specific AGE-binding receptors. The physiological consequences of AGEs in ageing and in the etiology of diabetic complications ${ }^{1}$ and in the development of age-related diseases such as inflammation ${ }^{2}$, atherosclerosis ${ }^{3}$ and neurodegenerative disorders ${ }^{4}$ has been described.

Despite progress in this field, a more comprehensive understanding of the putative effects of AGEs in the pathophysiology of these diseases is needed. In addition, the measurement of AGEs may also help to identify patients with a high risk for poor outcome and may thus help in risk stratification ${ }^{5,6}$. However, progress in the field is hampered by the lack of an easy method for the detection of AGEs in the laboratory. We need a rapid, simple and reliable method for the detection of AGES.

AGEs have traditionally been detected by enzyme-linked immunosorbent assay $(E L I S A)^{7-13}$. For several reasons the use of antisera for quantitative immunoassays of protein-bound AGEs is questionable. Reproducibility and sensitivity of such an assay are not optimal, because the specificity of the antibodies is often difficult to define and, because of steric constraints, not all AGE epitopes on the protein may be available for interaction with the antibody ${ }^{9,11}$. Thus, AGE measurements with immunoassays should be interpreted with care. A better approach for the quantitative determination of specific AGEs in proteins is the use of a specific analytical technique. For the determination of AGE levels in both tissue and blood samples, high performance liquid chromatography (HPLC) measurements ${ }^{14-17}$ and several mass spectrometry methods have been developed including gas chromatography mass spectrometry $(\mathrm{GC}-\mathrm{MS})^{18,19}$, and liquid chromatography tandem mass spectrometry (LC-MS/MS) ${ }^{13,17,20,21}$. However, the simultaneous quantification of CM L, CEL and MG-H1 in plasma or tissue, using a highly sensitive, selective and rapid analytical method, has not been reported yet.

In this paper we describe an ultra-performance liquid chromatography tandem mass spectrometry method for the simultaneous determination of $\mathrm{CML}$, CEL and MG-H1 in plasma. This method is based on the derivatisation of CML, CEL and MG-H1 with 1-butanol in protein hydrolysate or the supernatant of deproteinized plasma. 


\section{Materials and methods}

\section{Materials}

Boric acid, sodium borohydride, trifluoroacetic acid (TFA) and chloroform were obtained from Sigma-Aldrich (Zwijndrecht, the Netherlands). Butanol and methanol were obtained from Baker (Deventer, the Netherlands). Sodium hydroxide and hydrochloric acid were obtained from M erck (Darmstadt, Germany). Water and acetonitrile were obtained from Biosolve BV (Valkenswaard, the Netherlands). CM L (99\%), $\left[{ }^{2} \mathrm{H}_{2}\right]-\mathrm{CM} L$ (95.7\%), CEL (98.6\%), $\left[{ }^{2} \mathrm{H}_{4}\right]$-CEL, M G-H1 (92.6\%) and $\left[{ }^{2} \mathrm{H}_{3}\right]-\mathrm{M} \mathrm{G}-\mathrm{H} 1$ (98.7\%) were obtained from Polypeptide. All other reagents and solvents were of analytical or ULC/MS grade.

\section{Sample preparation protein-bound AGEs}

Whole blood was collected in sterile EDTA tubes. After centrifugation plasma was aspirated and stored at $-80^{\circ} \mathrm{C}$ until analysis. Before analysis, plasma samples where thawed and mixed thoroughly. Twenty-five $\mu \mathrm{L}$ of plasma was mixed with $50 \mu \mathrm{L}$ water in a $4 \mathrm{ml}$ reaction vial. After addition of $200 \mu \mathrm{L}$ of $100 \mathrm{mmol} / \mathrm{L}$ sodium borohydride dissolved

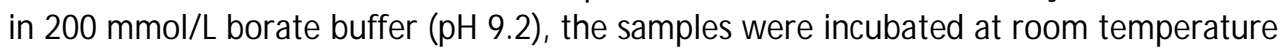
for 2 hours. Samples were mixed and subsequently deproteinized with $1000 \mu \mathrm{L}$ cold $\left(4^{\circ} \mathrm{C}\right)$ TFA. After centrifugation $\left(4300 \mathrm{~g}, 4^{\circ} \mathrm{C}, 20 \mathrm{~min}\right)$ the supernatant was carefully removed with a Pasteur pipette. For validation experiments $25 \mu \mathrm{L}$ of a standard solution (six point calibration curve; 5250-0 nmol/L CM L, 6250-0 nmol/L CEL and 14749-0 nmol/L M G-H1) was added. Samples were then hydrolyzed by adding $500 \mu \mathrm{L} 6 \mathrm{~N} \mathrm{HCl}$ and incubated for 24 hours at $110^{\circ} \mathrm{C}$. After hydrolysis $40 \mu \mathrm{L}$ hydrolysate and $20 \mu \mathrm{L}$ internal standard (containing $1432 \mathrm{nmol} / \mathrm{L}\left[{ }^{2} \mathrm{H}_{2}\right]-\mathrm{CM}$ L, $1378 \mathrm{nmol} / \mathrm{L}\left[{ }^{2} \mathrm{H}_{4}\right]$-CEL and $1322 \mathrm{nmol} / \mathrm{L}\left[{ }^{2} \mathrm{H}_{3}\right]-\mathrm{MG}-\mathrm{H} 1$ ) was mixed in a reaction vial. This mixture was evaporated to dryness under a stream of nitrogen gas at $70^{\circ} \mathrm{C}$ and subsequently derivatized in $100 \mu \mathrm{L} \mathrm{1-butanol:HCl}(3: 1, \mathrm{v} / \mathrm{v})$ for 90 minutes at $70^{\circ} \mathrm{C}$. Samples were then evaporated to dryness under nitrogen and redissolved in $200 \mu \mathrm{L}$ water.

\section{Sample preparation protein-bound lysine}

Sample preparation for protein-bound lysine analysis was performed as described above with the exception of the derivatisation-step. After acid hydrolysis $10 \mu \mathrm{L}$ hydrolysate was diluted with $800 \mu \mathrm{L}$ water. Twenty $\mu \mathrm{L}$ of this mixture and $20 \mu \mathrm{L}$ internal standard $\left[{ }^{13} \mathrm{C}_{6}\right]$-L-Lysine $(40.16 \mu \mathrm{mol} / \mathrm{L})$ was diluted with $500 \mu \mathrm{L} 10 \mathrm{mmol} / \mathrm{L}$ ammonia. 


\section{Sample preparation free AGES}

Twenty-five $\mu \mathrm{l}$ of internal standard (containing $716 \mathrm{nmol} / \mathrm{L}\left[{ }^{2} \mathrm{H}_{2}\right]-\mathrm{CML}, 689 \mathrm{nmol} / \mathrm{L}$ $\left[{ }^{2} \mathrm{H}_{4}\right]$-CEL and $661 \mathrm{nmol} / \mathrm{L}\left[{ }^{2} \mathrm{H}_{3}\right]-\mathrm{MG}$-H1) was mixed with $50 \mu$ l plasma. Samples were mixed thoroughly and subsequently deproteinized with $600 \mu \mathrm{l}$ of a mixture of methanol and acetonitrile (1:3, by volume) and centrifuged at $14000 \mathrm{rpm}$ for $20 \mathrm{~min}$ at room temperature. The supernatant was transferred to a reaction vial and further treated as described for the protein-bound AGEs.

\section{UPLC tandem MS analysis AGES}

Derivatized CML, CEL and MG-H1 were analyzed by ultra performance liquid chromatography (Acquity UPLC, Waters, Milford, USA) and detected in ESI positive multiple reaction monitoring (M RM) mode using a Xevo TQ MS (Waters, Milford, USA). Derivatives were separated on a reversed-phase $\mathrm{C} 18$ column (Acquity UPLC BEH C18, $50 \times 2.1 \mathrm{~mm}, 1.7 \mu \mathrm{m}$ ) with a linear gradient of $5 \mathrm{mmol} / \mathrm{L}$ ammonia and acetonitril at $48^{\circ} \mathrm{C}$. The flow rate was $800 \mu \mathrm{L} / \mathrm{min}$ and the injection volume was $2 \mu \mathrm{L}$. Quantification of $\mathrm{CML}$, CEL and MG-H1 was performed by calculating the peak area ratio of each unlabeled peak area to the corresponding internal standard peak area. The M RM transitions for CML, CEL and MG-H1 were respectively 317.1>186.1, 331.1>186.1 and 285.1>172.1. The MRM transitions for the internal standards $\left[{ }^{2} \mathrm{H}_{2}\right]-\mathrm{CML}$, $\left[{ }^{2} \mathrm{H}_{4}\right]-\mathrm{CEL}$, and $\left[{ }^{2} \mathrm{H}_{3}\right]-\mathrm{MG}-\mathrm{H} 1$ were respectively $319.1>186.1,335.1>190.1$ and $288.1>172.1$. Electrospray ionization was done at a capillary voltage of $0.5 \mathrm{kV}$ a source temperature of $150^{\circ} \mathrm{C}$ and a desolvation temperature of $600^{\circ} \mathrm{C}$. For qualitative and quantitative analysis, Masslynx software (V4.1, SCN 644, Waters) was used.

\section{UPLC tandem MS analysis lysine}

Protein bound AGEs were expressed in AGEs nmol/mmol LYS as per amount of protein. Therefore, underivatized lysine was analyzed by ultra performance liquid chromatography (Acquity UPLC, Waters, Milford, USA) and detected in ESI positive multiple reaction monitoring (M RM) mode using a Xevo TQ MS (Waters, Milford, USA). Lysine was analyzed on a reversed-phase $\mathrm{C} 18$ column (Acquity UPLC BEH $\mathrm{C} 18,50 \times 2.1 \mathrm{~mm}, 1.7 \mu \mathrm{m}$ ) with a linear gradient of $20 \mathrm{mmol} / \mathrm{L}$ ammonium formate and acetonitril at $30^{\circ} \mathrm{C}$. The flow rate was $800 \mu \mathrm{L} / \mathrm{min}$ and the injection volume was $2 \mu \mathrm{L}$. Quantification of lysine was performed by calculating the peak area ratio of the unlabeled peak area to the corresponding internal standard peak area. The MRM transitions for lysine and $\left[{ }^{13} \mathrm{C}_{6}\right]$ - L-Lysine were respectively $147.2>84.2$ and $153.2>89.2$. Electrospray ionization was done at a capillary voltage of $0.5 \mathrm{kV}$ a source temperature of $150^{\circ} \mathrm{C}$ and a desolvation temperature of $600^{\circ} \mathrm{C}$. 


\section{M ethod validation AGES}

Linearity was determined by adding standard solution of CML, CEL and M G-H1 to water and eight different plasma samples. For protein-bound AGEs in plasma, a six-point calibration curve was prepared CML $(0-5250 \mathrm{nmol} / \mathrm{L}), \mathrm{CEL}(0-6250 \mathrm{nmol} / \mathrm{L})$ and MG-H1 $(0-14750 \mathrm{nmol} / \mathrm{L})$. For free AGEs in plasma, a six-point calibration curve was prepared CML (0-525 nmol/L), CEL (0-625 nmol/L) and M G-H1 (0-1475 nmol/L). The peak area ratio of $\mathrm{CML}, \mathrm{CEL}$ and M G-H1 multiplied by the concentration of each corresponding internal standard were plotted as a function of the concentration. For evaluation of inter- and intra-assay variation, a pooled EDTA plasma sample was analyzed on 8 different days (inter-assay) and 8 times on the same day (intra-assay). To investigate potential differences in protein-bound AGE concentration as measured in plasma and serum samples blood from 8 healthy volunteers was collected in anticoagulant (citrate and EDTA) and plain (serum) tubes.

\section{Acid stability of AGES}

For protein-bound AGE analysis an acid hydrolysis is needed. To investigate acid stability of $\mathrm{CML}, \mathrm{CEL}$ and MG-H1 a six-point calibration curve was prepared as described in the previous paragraph and subsequently hydrolyzed. This calibration curve was compared to a calibration curve without acid hydrolysis.

\section{Method validation lysine}

Linearity was determined by adding standard solution of lysine to water and 9 different plasma samples. A six-point calibration curve was prepared for lysine (0-198.2 $\mu \mathrm{mol} / \mathrm{L})$. The peak area ratio of lysine multiplied by the concentration of $\left[{ }^{13} \mathrm{C}_{6}\right]$-L-Lysine were plotted as a function of the concentration. For evaluation of inter- and intra-assay variation, a pooled EDTA plasma sample was analyzed on 11 different days (inter-assay) and 6 times on the same day (intra-assay). Method correlation, of the described lysine analysis, was tested in 44 protein-lysate samples as compared with a Pierce bicinchoninic acid (BCA) protein assay kit (Thermo Scientific, Rockford, USA). 


\section{Results}

\section{UPLC tandem MS analysis AGEs and lysine}

A representative chromatogram of free AGEs in an EDTA pool plasma, protein-bound AGEs in an EDTA pool plasma and a standard in water is shown in figure 2B.1 A, B and C. Retention times of CM L, CEL and M G-H1 were $9.05,9.70$ and 3.71 minutes, respectively, and the corresponding stable isotopes were eluted at 9.03, 9.68 and 3.69, respectively. Retention time of lysine and the corresponding stable isotope was 0.17 minutes (chromatogram not shown).
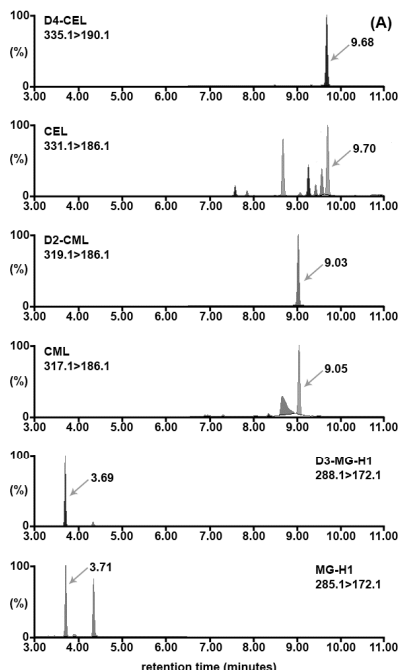
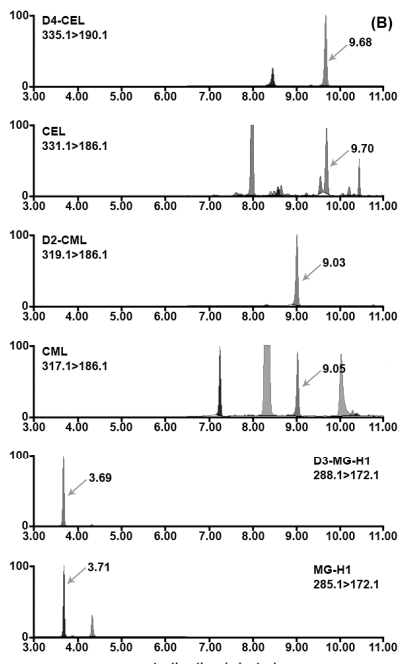
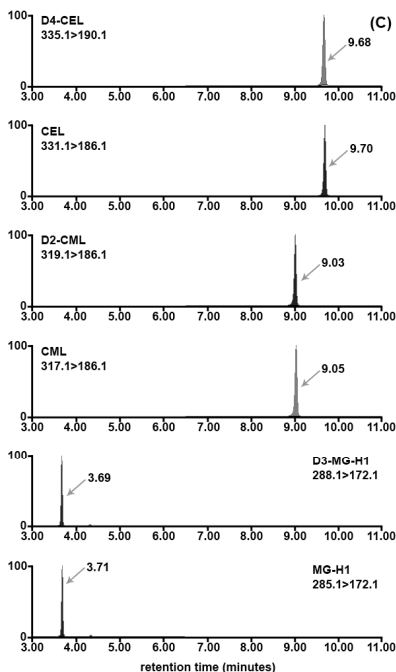

Figure 2B.1. Representative chromatograms of a standard solution of AGEs (C), an EDTA plasma sample of free AGEs (A) and an EDTA plasma sample of protein-bound AGEs (B). The elution of the AGEs are indicated with an arrow mark and corresponding retention time. Concentrations of $\mathrm{CM} \mathrm{L,} \mathrm{CEL} \mathrm{and} \mathrm{M} \mathrm{G-H1} \mathrm{in} \mathrm{the} \mathrm{standard} \mathrm{solution}$ (C) were 5250, 6250 and $14750 \mathrm{nmol} / \mathrm{L}$, in the EDTA plasma free form (A) were 65.7, 44.6 and $329.0 \mathrm{nmol} / \mathrm{L}$ and in the EDTA plasma protein-bound form (B) were 3269, 926 and $4354 \mathrm{nmol} / \mathrm{L}$, respectively.

\section{Method validation AGE's}

The calibration curves for CML, CEL and MG-H1 were linear over the described concentration ranges $(r 2>0.99)$ in both water and plasma matrix. Mean slope (responsefactor) for protein-bound CML, CEL and M G-H1 as tested in 8 different matrices were respectively 1.064 (CV, 8.9\%), 1.429 (CV, 6.0\%) and 0.5718 (CV, 13.5\%). M ean slope for free CML, CEL and MG-H1 as tested in 8 different matrices were respectively 1.050 (CV, 10.0\%), 1.463 (CV, 3.1\%) and 1.010 (CV, 3.8\%).

Inter- and intra-assay variation as determined by replicate analysis of a plasma sample was given in table 2B.1. 


\begin{tabular}{|c|c|c|c|c|c|c|c|c|}
\hline \multirow[b]{3}{*}{ Compound } & \multicolumn{4}{|c|}{ Intra-assay variation $(n=8)$} & \multicolumn{4}{|c|}{ Inter-assay variation $(n=8)$} \\
\hline & \multicolumn{2}{|c|}{ Protein-bound } & \multicolumn{2}{|c|}{ Free } & \multicolumn{2}{|c|}{ Protein-bound } & \multicolumn{2}{|c|}{ Free } \\
\hline & $\begin{array}{c}\text { Mean (SD) } \\
\text { nmol/L }\end{array}$ & CV \% & $\begin{array}{c}\text { Mean (SD) } \\
\text { nmol/L }\end{array}$ & CV \% & $\begin{array}{c}\text { Mean (SD) } \\
\text { nmol/ L }\end{array}$ & CV \% & $\begin{array}{c}\text { Mean (SD) } \\
\text { nmol/L }\end{array}$ & CV \% \\
\hline$\overline{\mathrm{CML}}$ & 3990 (176) & 4.4 & $66.1(1.8)$ & 2.8 & 3895 (174) & 4.5 & $70.6(5.0)$ & 7.1 \\
\hline CEL & $1278(62)$ & 4.8 & $46.9(1.7)$ & 3.7 & 1251 (182) & 14.6 & $43.5(2.8)$ & 6.4 \\
\hline M G-H1 & 12072 (1005) & 8.3 & $348.4(13.0)$ & 3.7 & $12976(3067)$ & 23.6 & $337.5(17.1)$ & 5.1 \\
\hline
\end{tabular}

Table 2B.1. Intra- and inter-assay variation of protein-bound and free AGEs as determined by replicate analysis of a plasma sample.

The lower limits of quantification ( $\mathrm{s} / \mathrm{N}=10$ ) on column for protein-bound $\mathrm{CML}, \mathrm{CEL}$ and MG-H1 were 4, 4, and $7 \mathrm{fmol}$, corresponding to a concentration of 200, 200 and 340 $\mathrm{nmol} / \mathrm{L}$. The lower limits of quantification $(\mathrm{s} / \mathrm{N}=10)$ on column for free $\mathrm{CML}, \mathrm{CEL}$ and MG-H1 were 4,3 and $6 \mathrm{fmol}$, corresponding to a concentration of $8.5,4.9$ and $11.8 \mathrm{nmol} / \mathrm{L}$.

Mean protein-bound AGE concentrations, as measured in 8 different plasma and serum samples, were shown in figure 2B.2. Mean protein-bound $\mathrm{CML}$ concentration, as measured in citrate-, EDTA plasma and serum, was 2578, 3353 and $3026 \mathrm{nmol} / \mathrm{L}$, respectively. Mean protein-bound CEL concentration, as measured in citrate-, EDTA plasma and serum, was 877.8, 1315 and $1008 \mathrm{nmol} / \mathrm{L}$, respectively. Mean protein-bound MG-H1 concentration, as measured in citrate-, EDTA plasma and serum, was 12332, 13115 and $11937 \mathrm{nmol} / \mathrm{L}$, respectively. We found higher CM L and CEL concentrations, as measured in EDTA plasma compared to citrate plasma and serum. However, this was only significant for CEL $(p<0.05)$. For $C M L$ we observed only a significant difference between EDTA- and citrate plasma $(p<0.05)$. No statistically significant differences were found between citrate- EDTA plasma and serum M G-H1 concentrations.
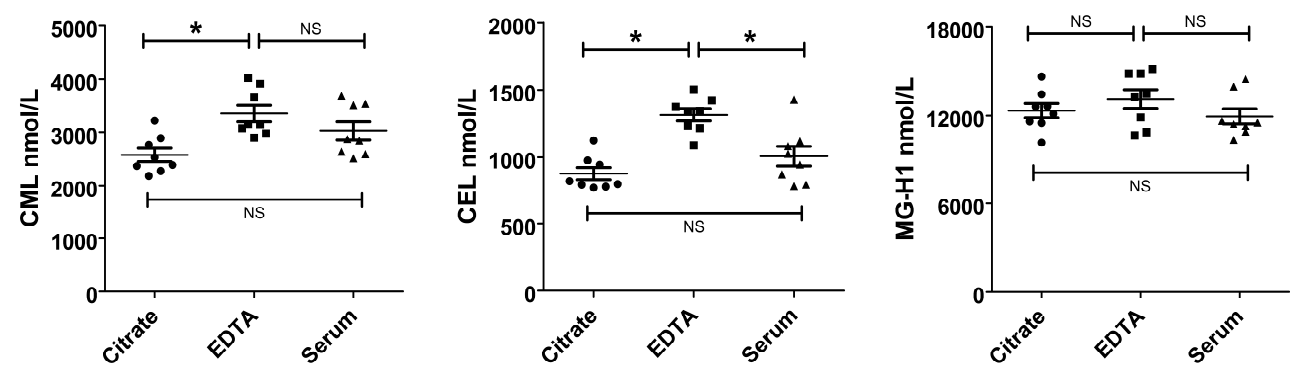

Figure 2B.2. Mean protein-bound AGE concentrations, as measured in 8 different plasma and serum samples. Data are expressed as mean \pm SEM and separate datapoints (One-way ANOVA, ${ }^{*}=p \varangle 0.05$, NS $=$ not significant).

\section{Acid stability of AGEs}

The calibration curves for CML, CEL and M G-H1, as tested in water after acid hydrolysis, were compared to the calibration curves without acid hydrolysis. The slope for CM L, CEL and MG-H1 as tested in water, without acid hydrolysis, was 1.18, 1.59 and 1.03, respectively. For CML and CEL no differences were observed between the mean slopes of the calibration curve with or without acid hydrolysis (figure 2B.3). 
For M G-H1, however, a decrease of the mean slope from 1.03 to 0.56 was observed after acid hydrolysis (figure 2B.3). Thus, under these standard conditions we found a recovery of M G-H1 of $\sim 56 \%$.
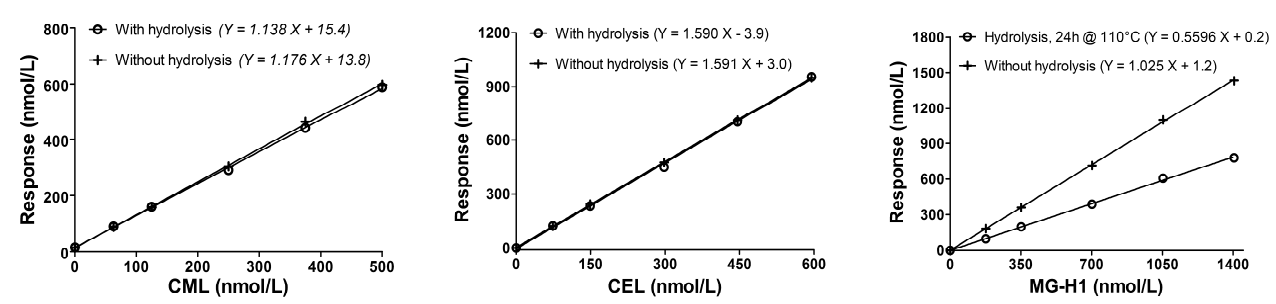

Figure 2B.3. Calibration curves in water with and without hydrolysis.

\section{Method validation lysine}

The calibration curve for lysine was linear over the described concentration range $\left(r^{2}>0.99\right)$ in both water and plasma matrix. M ean slope (responsefactor) for lysine tested in 9 different matrices was 0.9936 (CV, 4.2\%). Inter-assay variation as determined by replicate analysis of a plasma sample on 11 different days was 4.9 (CV\%). Intra-assay variation as determined by replicate analysis $(n=6)$ of a plasma sample on one day was $2.7(\mathrm{CV} \%)$. The lower limits of quantification $(\mathrm{s} / \mathrm{N}=10)$ on column for lysine was $11 \mathrm{fmol}$, corresponding to a concentration of $254 \mu \mathrm{mol} / \mathrm{L}$. The described lysine analysis gave good correlation with the protein analysis as tested in 44 different protein-lysate samples $\left(r^{2}=0.9304\right.$, figure 2B.4).

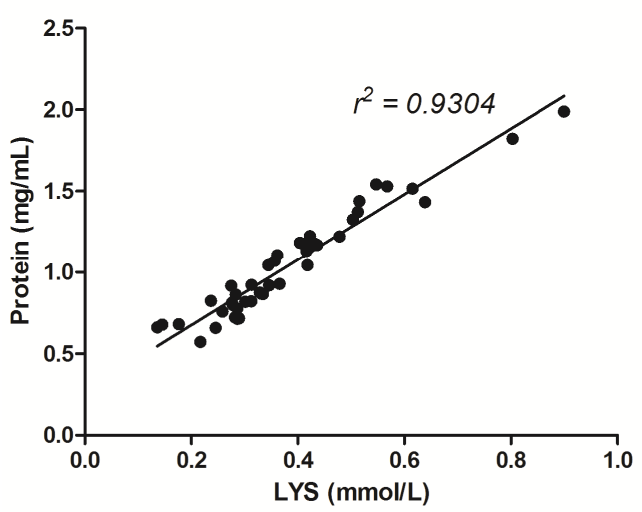

Figure 2B.4. M ethod correlation, as tested in 44 protein-lysate samples. Lysine concentrations correlated with protein concentrations as analyzed with a Pierce bicinchoninic acid (BCA) protein assay kit. 


\section{UPLC tandem MS analysis application}

The described method has been applied to analyze human and animal plasma samples originating from a broad range of clinical trials and animal studies ${ }^{22-29}$. Plasma AGE

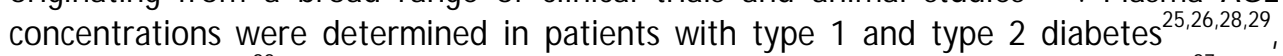
obese individual $\mathrm{s}^{22}$ and patients with chronic obstructive pulmonary disease (COPD) ${ }^{27}$.

As an illustration, we analyzed free and protein-bound AGEs in healthy volunteers and in patients with end stage renal disease (ESRD) receiving peritoneal dialysis (PD). As described earlier, higher plasma concentrations of protein-bound pentosidine were measured in patients with ESRD and compared to healthy volunteers ${ }^{30}$. Moreover, higher plasma concentrations of protein-bound and free AGEs were found in patients with ESRD compared to healthy controls ${ }^{21,31}$. We analyzed protein-bound AGEs in 8 healthy volunteers and in 8 patients on peritoneal dialysis. The mean concentrations of proteinbound plasma CML, CEL and M G-H1 in the PD patients were 13832, 2762 and 14105 $\mathrm{nmol} / \mathrm{L}$, respectively and were higher than in healthy volunteers 3129, 1902 and 7800 $\mathrm{nmol} / \mathrm{L}$, respectively (fig $2 \mathrm{~B} .5 \mathrm{~A})$. This was significant for $\mathrm{CML}$ and M G-H1 $(\mathrm{p}<0.0001)$ but not for CEL ( $p=0.1125$ ). We analyzed free AGEs in 9 healthy volunteers and in 9 patients on peritoneal dialysis. The mean concentrations of free plasma CML, CEL and MG-H1 in the PD patients were 1084,1345 and $4161 \mathrm{nmol} / \mathrm{L}$, respectively and were significantly higher $(p \varangle 0.0001)$ than in healthy volunteers 68,105 and $409 \mathrm{nmol} / \mathrm{L}$, respectively (figure 2B.5B).
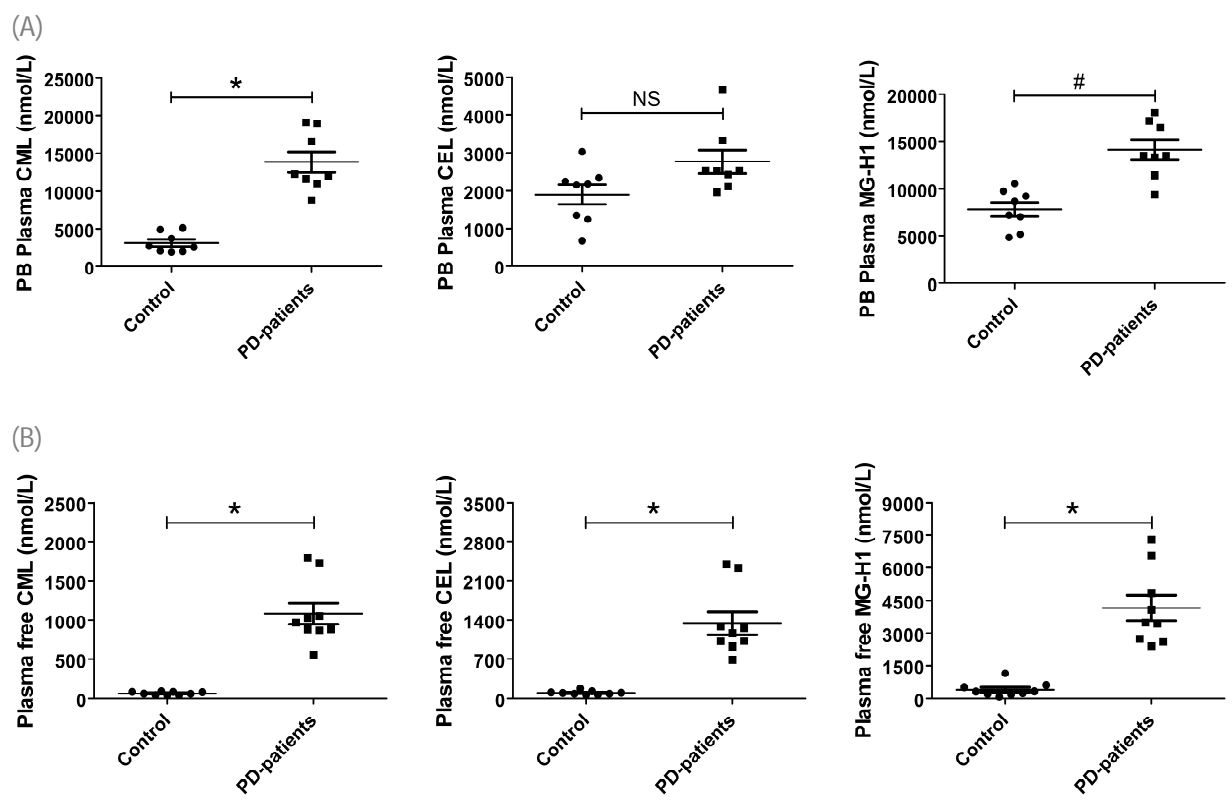

Figure 2B.5. Protein-bound (A) and free (B) CML, CEL and MG-H1 concentrations in plasma of healthy controls and in peritoneal dialysis (PD) patients. Data are expressed as mean \pm SEM and separate datapoints (Unpaired ttest, $*=p \varangle 0.0001$, NS = not significant, $\#=p=0.0002$ ). 


\section{Discussion}

In 1912, Louis Camille Maillard described for the first time the reaction of protein with glucose to form advanced glycation endproduct ${ }^{32}$. Since his discovery a lot of research was done to investigate AGEs in the human body and their role in several diseases. To quantify AGEs in plasma and other matrices, many techniques have been described, including ELISA ${ }^{7-13}, \mathrm{HPLC}^{14-17}, \mathrm{GC}-\mathrm{MS} \mathrm{S}^{19,33}$ and LC-M S ${ }^{13,17,20,21}$, however with some shortcomings. ELISA based techniques have several limitations quantitatively. Standardization of this method is difficult, immunoreactivity of the antibody and AGE epitope can be affected by numerous factors and sensitivity and specificity is often lacking ${ }^{9}$. HPLC and LC-MS uses elaborate and expensive enzymatic hydrolysis ${ }^{14,17,21}$ which is not desirable for large cohort studies. Moreover, several analytical methods analyzed just one or two $\mathrm{AGES}^{13,20,33}$.

We describe here a sensitive, simple and highly selective method to determine proteinbound and free CML, CEL and M G-H1 in plasma by UPLC tandem MS.

The described UPLC tandem MS method determines simultaneously CML, CEL and M G-H1 in acid hydrolyzed plasma protein. To express these data as AGE per amount of protein, the amino acid lysine was determined in the same hydrolysate. In particular, when processing tissue samples or cell lysates it is important to correct for the amount of processed protein. Moreover, pentosidine was determined in the same hydrolysate with HPLC-fluorescence detection as described earlier ${ }^{30}$.

To investigate the effect of anticoagulant on levels of protein-bound AGEs, citrate-, EDTA plasma and serum were considered. Protein-bound levels of CML and CEL, as measured in EDTA plasma, were higher compared to citrate plasma and serum. No differences were found between citrate-, EDTA plasma and serum levels of M G-H1. These differences are most likely due to an ion suppressing or enhancing effect on the response of these polar metabolites caused by the anticoagulant, in particular citrate and EDTA ${ }^{34}$.

CML, CEL and MG-H1 were also determined as the free-form in the supernatant of deproteinized plasma. Twenty five $\mu \mathrm{L}$ of plasma was used for the analysis of proteinbound AGEs and $50 \mu \mathrm{L}$ plasma was used to determine free AGEs. For each compound of interest a stable isotope internal standard was used. In this way determination is more accurate since the use of these stable isotope internal standards correct for possible coeluting polar contaminants that cause ion suppression. Moreover, a derivatisation with 1-butanol was used to gain more retention for polar compounds on reversed-phase chromatography and increases sensitivity during electro-spray ionization.

With the described method we analyzed plasma free and protein-bound AGEs in healthy volunteers and in patients with end stage renal disease (ESRD) receiving peritoneal dialysis (PD). We found increased plasma levels of both free and protein-bound AGEs in patients with ESRD as compared to healthy controls, which is in reasonable agreement with data described in literature ${ }^{20,35-39}$. However, absolute plasma levels differ between studies. 
Differences in sample preparation, most likely protein precipitation by acid solutions compared to ultrafiltration of the plasma ${ }^{21,39}$ and protein-bound AGEs released as free adducts via acid hydrolysis or enzymatic hydrolysis ${ }^{21}$ can lead to different yields of AGEs. Besides, the use of standards synthetized in own laboratory facilities compared to commercially available standards can lead to absolute differences in concentrations between studies.

In conclusion, the described method proofed to be suitable in studying AGEs in several different human and animal studies ${ }^{22-29}$ and help us to understand the putative effects of AGEs in the pathophysiology of different diseases. 


\section{References}

Goldin, A., Beckman, J. A., Schmidt, A. M . \& Creager, M. A. Advanced glycation end products: sparking the development of diabetic vascular injury. Circulation 114, 597-605 (2006).

Yan, S. F., Ramasamy, R. \& Schmidt, A. M. Mechanisms of disease: advanced glycation end-products and their receptor in inflammation and diabetes complications. Nat Clin Pract Endocrinol Metab 4, 285-293 (2008).

3 Basta, G., Schmidt, A. M. \& De Caterina, R. Advanced glycation end products and vascular inflammation: implications for accelerated atherosclerosis in diabetes. Cardiovasc Res 63, 582-592 (2004).

Takeuchi, M. \& Yamagishi, S. Possible involvement of advanced glycation end-products (AGEs) in the pathogenesis of Alzheimer's disease. Curr Pharm Des 14, 973-978 (2008).

Kilhovd, B. K. et al. High serum levels of advanced glycation end products predict increased coronary heart disease mortality in nondiabetic women but not in nondiabetic men: a population-based 18year follow-up study. Arterioscler Thromb Vasc Biol 25, 815-820 (2005).

Koyama, Y. et al. High serum level of pentosidine, an advanced glycation end product (AGE), is a risk factor of patients with heart failure. J Card Fail 13, 199-206 (2007).

Sanaka, T. et al. Plasma pentosidine levels measured by a newly developed method using ELISA in patients with chronic renal failure. Nephron 91, 64-73 (2002).

Izuhara, Y. et al. A sensitive and specific ELISA for plasma pentosidine. Nephrol Dial Transplant 14, 576-580 (1999)

Mitsuhashi, T., Vlassara, H., Founds, H. W. \& Li, Y. M. Standardizing the immunological measurement of advanced glycation endproducts using normal human serum. J Immunol Methods 207, 79-88 (1997).

Munch, G. et al. Determination of advanced glycation end products in serum by fluorescence spectroscopy and competitive ELISA. European journal of clinical chemistry and clinical biochemistry : journal of the Forum of European Clinical Chemistry Societies 35, 669-677 (1997).

Dorrian, C. A., Cathcart, S., Clausen, J., Shapiro, D. \& Dominiczak, M. H. Factors in human serum interfere with the measurement of advanced glycation endproducts. Cellular and molecular biology 44, 1069-1079 (1998).

Takayama, F. et al. Immunohistochemical detection of imidazolone and $\mathrm{N}$ (epsilon)(carboxymethyl)lysine in aortas of hemodialysis patients. Cellular and molecular biology 44, 11011109 (1998).

Srey, C. et al. Immunochemical and mass spectrometric analysis of Nepsilon-(carboxymethyl)lysine content of AGE-BSA systems prepared with and without selected antiglycation agents. Journal of agricultural and food chemistry 58, 11955-11961, doi:10.1021/jf102543g (2010).

Ahmed, N., Argirov, O. K., M inhas, H. S., Cordeiro, C. A. \& Thornalley, P. J. Assay of advanced glycation endproducts (AGEs): surveying AGEs by chromatographic assay with derivatization by 6aminoquinolyl-N-hydroxysuccinimidyl-carbamate and application to Nepsilon-carboxymethyl-lysineand Nepsilon-(1-carboxyethyl)lysine-modified albumin. Biochem J 364, 1-14 (2002). 
Ahmed, N. \& Thornalley, P. J. Chromatographic assay of glycation adducts in human serum albumin glycated in vitro by derivatization with 6 -aminoquinolyl-N-hydroxysuccinimidyl-carbamate and intrinsic fluorescence. Biochem J 364, 15-24 (2002).

Hartkopf, J. \& Pahlke, C. Determination of N-carboxymethyllysine by a reversed-phase highperformance liquid chromatography method. J Chromatogr A 672, 242-246 (1994).

Ahmed, M. U., Thorpe, S. R. \& Baynes, J. W. Identification of $\mathrm{N}$ epsilon-carboxymethyllysine as a degradation product of fructoselysine in glycated protein. J Biol Chem 261, 4889-4894 (1986).

Charissou, A., Ait-Ameur, L. \& Birlouez-Aragon, I. Evaluation of a gas chromatography/mass spectrometry method for the quantification of carboxymethyllysine in food samples. Journal of chromatography. A 1140, 189-194, doi:10.1016/j.chroma.2006.11.066 (2007).

Anderson, M. M. \& Heinecke, J. W. Production of N(epsilon)-(carboxymethyl)lysine is impaired in mice deficient in NADPH oxidase: a role for phagocyte-derived oxidants in the formation of advanced glycation end products during inflammation. Diabetes 52, 2137-2143 (2003).

Teerlink, T., Barto, R., Ten Brink, H. J. \& Schalkwijk, C. G. Measurement of Nepsilon(carboxymethyl)lysine and Nepsilon-(carboxyethyl)lysine in human plasma protein by stable-isotopedilution tandem mass spectrometry. Clin Chem 50, 1222-1228 (2004).

Thornalley, P. J. et al. Quantitative screening of advanced glycation endproducts in cellular and extracellular proteins by tandem mass spectrometry. Biochem J 375, 581-592 (2003).

Gaens, K. H. et al. Nepsilon-(carboxymethyl)lysine-receptor for advanced glycation end product axis is a key modulator of obesity-induced dysregulation of adipokine expression and insulin resistance. Arterioscler Thromb Vasc Biol 34, 1199-1208, doi:10.1161/ATVBAHA.113.302281 (2014).

Gaens, K. H. et al. Endogenous formation of Nepsilon-(carboxymethyl)lysine is increased in fatty livers and induces inflammatory markers in an in vitro model of hepatic steatosis. J Hepatol 56, 647-655, doi:10.1016/j.jhep.2011.07.028 (2012).

Brouwers, 0 . et al. Overexpression of glyoxalase-I reduces hyperglycemia-induced levels of advanced glycation endproducts and oxidative stress in diabetic rats. J Biol Chem (2011).

van Eupen, M. G. et al. Plasma levels of advanced glycation endproducts are associated with type 1 diabetes and coronary artery calcification. Cardiovasc Diabetol 12, 149, doi:10.1186/1475-2840-12149 (2013).

Nin, J. W. et al. Higher plasma levels of advanced glycation end products are associated with incident cardiovascular disease and all-cause mortality in type 1 diabetes: a 12-year follow-up study. Diabetes Care 34, 442-447, doi:10.2337/dc10-1087 (2011).

Gopal, P. et al. Plasma advanced glycation end-products and skin autofluorescence are increased in COPD. The European respiratory journal 43, 430-438, doi:10.1183/09031936.00135312 (2014).

Hanssen NM, Engelen L, Ferreira I, Scheijen JL, Huijberts MS, van Greevenbroek MM, et al. Plasma levels of advanced glycation endproducts are not independently associated with cardiovascular disease in individuals with or without type 2 diabetes: the Hoorn and CODAM Studies. J Clin Endocrinol M etab (2013).

Hanssen, N. M. et al. Plasma advanced glycation endproducts are associated with incident cardiovascular events in individuals with type 2 diabetes: a case-cohort study with a median followup of 10 years (EPIC-NL). Diabetes, doi:10.2337/db13-1864 (2014). 
Scheijen, J. L., van de Waarenburg, M. P., Stehouwer, C. D. \& Schalkwijk, C. G. Measurement of pentosidine in human plasma protein by a single-column high-performance liquid chromatography method with fluorescence detection. Journal of chromatography. B, Analytical technologies in the biomedical and life sciences 877, 610-614, doi:10.1016/j.jchromb.2009.01.022 (2009).

31 Makita, Z. et al. Advanced glycosylation end products in patients with diabetic nephropathy. The New England journal of medicine 325, 836-842, doi:10.1056/NEJM 199109193251202 (1991).

32 Maillard, L. C. Action des acides aminés sur les sucres: formation des mélanoidines par voie méthodique. C.R. Acad. Sci. 154, 66-68 (1912).

33 Charissou, A., Ait-Ameur, L. \& Birlouez-Aragon, I. Evaluation of a gas chromatography/mass spectrometry method for the quantification of carboxymethyllysine in food samples. J Chromatogr A 1140, 189-194, doi:10.1016/j.chroma.2006.11.066 (2007).

Barri, T. \& Dragsted, L. O. UPLC-ESI-QTOF/MS and multivariate data analysis for blood plasma and serum metabolomics: effect of experimental artefacts and anticoagulant. Anal Chim Acta 768, 118128, doi:10.1016/j.aca.2013.01.015 (2013).

35 Degenhardt, T. P. et al. Technical note. The serum concentration of the advanced glycation endproduct N epsilon-(carboxymethyl)lysine is increased in uremia. Kidney Int 52, 1064-1067 (1997). Weiss, M. F. et al. Mechanisms for the formation of glycoxidation products in end-stage renal disease. Kidney Int 57, 2571-2585, doi:10.1046/j.1523-1755.2000.00117.x (2000). Agalou, S., Ahmed, N., Dawnay, A. \& Thornalley, P. J. Removal of advanced glycation end products in clinical renal failure by peritoneal dialysis and haemodialysis. Biochem Soc Trans 31, 1394-1396 (2003).

38 Thornalley, P. J. Glycation free adduct accumulation in renal disease: the new AGE. Pediatr Nephrol 20, 1515-1522 (2005).

39 Agalou, S., Ahmed, N., Thornalley, P. J. \& Dawnay, A. Advanced glycation end product free adducts are cleared by dialysis. Ann N Y Acad Sci 1043, 734-739 (2005). 
PLASMA LEVELS OF ADVANCED CLYCATION ENDPRODUCTS Ne-(carboxymethyl) lysine, $\mathrm{N \varepsilon}$-(carboxyethyl) lysine and pentosidine ARENOT INDEPENDENILY ASSOCIATED WHH CARDIOVASCULAR DIS AASE IN INDIVIDUALS - WITHOR WIHHOUT Wype 2 diabetes:

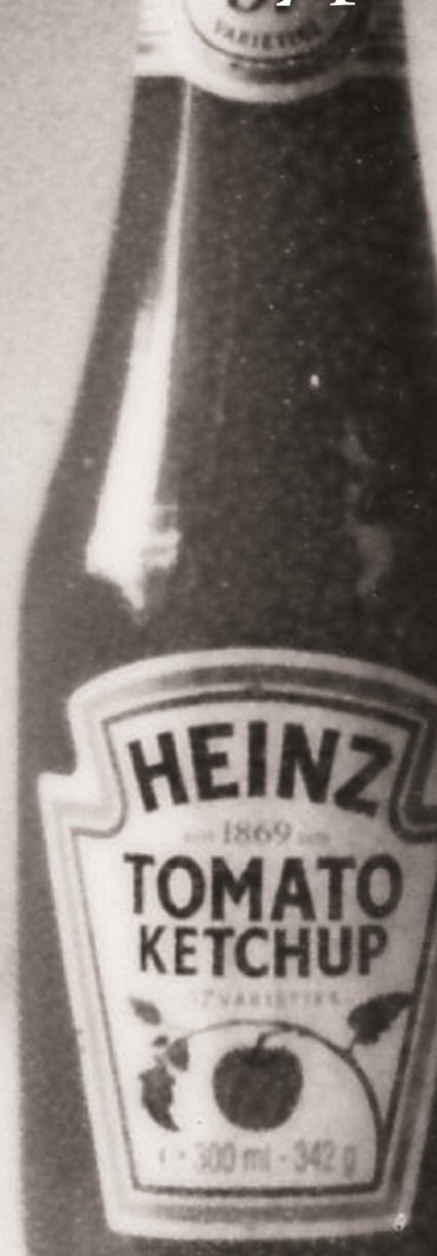
THE HOORN AND CODAM SIUDIES 


\section{3}

Plasma levels of advanced glycation endproducts $\mathrm{N}^{\varepsilon}$-(carboxymethyl)lysine, $\mathrm{N}^{\varepsilon}$-(carboxyethyl)lysine and pentosidine are not independently associated with cardiovascular disease in individuals with or without type 2 diabetes: the Hoorn and CODAM Studies

Nordin M .J. Hanssen*, Lian Engelen*, Isabel Ferreira, Jean L.J.M. Scheijen, M aya S. Huijberts, M arleen M .J. van Greevenbroek, Carla J.H. van der Kallen, Jacqueline M. Dekker, Giel Nijpels, Coen D.A. Stehouwer, Casper G. Schalkwijk

Journal of clinical endocrinology and metabolism 98:8; (2013) 


\section{Abstract}

\section{Objective}

Experimental and histological data suggest a role for advanced glycation endproducts (AGEs) in cardiovascular disease (CVD), particularly in type 2 diabetes (T2DM ). However, the epidemiological evidence of an adverse association between AGEs and CVD remains inconclusive. We therefore investigated, in individuals with various degrees of glucose metabolism, the associations of plasma AGEs with prevalent CVD.

\section{Research design and Methods}

We measured plasma levels of protein-bound $\mathrm{N}^{\varepsilon}$-(carboxymethyl)lysine (CML), $\mathrm{N}^{\varepsilon}$-(carboxyethyl)lysine (CEL) and pentosidine, in participants from two Dutch cohort studies ( $n=1291$, mean age $64.7 \pm 8.3$ years, $45 \%$ women), including 573 individuals with normal glucose metabolism (NGM), 304 with impaired glucose metabolism (IGM) and 414 with T2DM . Additionally, we measured free CM L, CEL and 5-hydro-5-methylimidazolone (MG-H1) in a subset of participants $(n=554)$. Data were analyzed with multiple logistic or linear regression analyses.

\section{Results}

Protein-bound levels of CEL [32 (IQR: $25-40$ vs. 28 (22-35) nmol/mmol lysine] and pentosidine [0.53 $(0.43-0.67)$ vs. $0.48(0.40-0.59) \mathrm{nmol} / \mathrm{mmol}$ lysine] as well as free CEL [48 (39-62) vs. 45 (36-56) nmol/L] and MG-H1 [141 (96-209) vs. 116 (84-165) nmol/L] were higher in individuals with vs. without CVD, whereas protein-bound CML was lower [33 (27-38) vs. 34 (29 39) $\mathrm{nmol} / \mathrm{mmol}$ lysine]. However, these differences disappeared after adjustment for confounders. The associations did not differ consistently between individuals with- and without T2DM.

\section{Conclusion}

We found no independent adverse associations of plasma AGEs with CVD in individuals with NGM, IGM and T2DM. 


\section{Introduction}

Evidence from experimental studies links advanced glycation endproducts (AGEs), a heterogeneous family of sugar-modified proteins, to cardiovascular disease (CVD) and other complications in diabetes ${ }^{1}$. However, epidemiological evidence of an adverse association between plasma AGEs and CVD, in individuals with or without type 2 diabetes (T2DM), remains uncertain, as studies have yielded both positive ${ }^{2-7}$ and lack of associations ${ }^{8-10}$. Several factors may explain these conflicting results. Most studies were rather small, did not adjust for potential confounders, used semi-quantitative ELISAtechniques or did not measure protein-bound and free circulating forms of AGES separately, potentially confounding results.

Therefore, we investigated, in a large combined sample of participants from two similar cohort studies: firstly, the extent to which plasma levels of the AGEs protein-bound $\mathrm{N}^{\varepsilon}$-(carboxymethyl)lysine (CML), $\mathrm{N}^{\varepsilon}$-(carboxyethyl)lysine (CEL) and pentosidine, measured with state-of-the-art (ultra-performance) liquid chromatography-tandem MS (UPLC-M S/MS) or HPLC ${ }^{11}$, differed across different levels of impaired glucose metabolism [i.e. normal (NGM), impaired (IGM) and T2DM], and secondly, whether these AGEs were associated with prevalent CVD. In addition, similar analyses were conducted for free levels of CM L, CEL and 5-hydro-5-methylimidazolone (M G-H1) in a subset of participants. Finally, we investigated whether these associations differed between individuals withand without diabetes.

\section{Research design and methods}

A brief summary of the design and methods of this study is presented below, and a full presentation of the methods is available in the online supplement, published on The Endocrine Society's J ournals Online web site at http://jcem.endojournals.org.

\section{Study population}

The present study included participants from the baseline examination of the Cohort on Diabetes and Atherosclerosis Maastricht (CODAM) Study ${ }^{12}$ and from the follow-up examination of the Hoorn Study ${ }^{13}$. These studies were designed to allow future pooling. Individuals missing data on plasma AGEs $(n=88)$ or CVD status $(n=17)$ were excluded from present analyses, resulting in a total of 1,291 individuals (554 from CODAM and 737 from Hoorn). Written informed consent was obtained from all participants and both cohort studies were approved by the local M edical Ethics Committees. 


\section{M easurement of plasma AGEs}

Plasma AGEs were measured in EDTA plasma samples, obtained from fasting venous blood, which were stored at $-80^{\circ} \mathrm{C}$ until analysis. We developed a new method to determine protein-bound $\mathrm{CML}$ and $\mathrm{CEL}$ and free $\mathrm{CML}, \mathrm{CEL}$ and $\mathrm{MG}-\mathrm{H} 1$ in plasma, using UPLC-tandem MS, which we describe in detail in Chapter 2B.

\section{Definition of glucose metabolism status}

In both cohorts participants' glucose metabolism status (GMS) was ascertained on the basis of fasting and 2-hour post-load glucose levels according to the WHO's criteria of 1999 , as described in detail elsewhere ${ }^{12,13}$. In short, individuals with normal fasting $(\varangle 6.1 \mathrm{mmol} / \mathrm{L})$ and 2-hour post-load $(<7.8 \mathrm{mmol} / \mathrm{L})$ glucose levels were classified as having NGM. Individuals with impaired fasting glucose $(6.1-7.0 \mathrm{mmol} / \mathrm{L})$, impaired glucose tolerance (post-load glucose 7.8-11.1 $\mathrm{mmol} / \mathrm{L}$ ) or both were classified as having IGM. Individuals using glucose lowering medication, insulin, and/or with a fasting plasma glucose of $\geq 7.0 \mathrm{mmol} / \mathrm{L}$ and/or 2 -hour post-load glucose of $\geq 11.1 \mathrm{mmol} / \mathrm{L}$ were classified as having T2DM.

\section{Definition of cardiovascular disease}

Prevalent CVD was defined as history of myocardial infarction, stroke or transient ischemic attack, non-traumatic limb amputation, coronary or peripheral artery bypass, angioplasty (self-reported), and/or signs of infarction or ischemia measured on a 12-lead ECG (M innesota codes 1-1 to 1-3, 4-1 to $4-3,5-1$ to $5-3$ or $7-1$ ) or an ankle brachial index of $\varangle 0.9$ in either leg ${ }^{12,13}$. Taken together, of the individuals with CVD, 83\% had coronary heart disease, $20 \%$ had cerebrovascular disease and $15 \%$ had peripheral arterial disease.

\section{Covariates}

Study covariates included waist circumference, smoking status, mean BP (M BP; calculated as $2 * \mathrm{DBP}+\mathrm{SBP}) / 3$ ), total, $\mathrm{HDL}$ and LDL cholesterol, triglycerides, estimated (e) GFR (using the short Modification of Diet in Renal Disease equation ${ }^{14}$ ), presence of micro- or macroalbuminuria and use of BP-, lipid- and glucose-lowering medication, and were determined as described earlier ${ }^{12,13}$.

\section{Statistical analyses}

All statistical analyses were carried out using the Predictive Analytics SoftWare, version 18.0 (SPSS IBM Corporation, Armonk, NY, USA). Skewed variables (i.e. CML, CEL, pentosidine, M G-H1, triglycerides) were $\log _{\mathrm{e}}$ transformed prior to all analyses. 


\section{Data analyses}

We used linear regression analyses to compare levels of AGEs between individuals with IGM or T2DM vs. NGM (reference group), first, after adjustments for age, sex, and cohort (model 1) and additionally for waist, smoking, MAP, blood lipids, eGFR, albuminuria and use of medication (model 2). We used logistic regression analyses to investigate the associations between plasma AGEs and prevalent CVD.

\section{Results}

General characteristics of the study population stratified according to prevalent CVD and GMS are shown in table 3.1.

\section{Comparison of plasma AGE levels by GMS}

Although crude plasma levels of protein-bound CML decreased and of protein-bound and free CEL increased with deteriorating GMS, (table 3.1), after adjustment for potential confounders (supplementary table S3.1), the levels of protein-bound CM L and free CEL no longer differed significantly across GMS. However, but only after full adjustment for study covariates, protein-bound pentosidine was higher in individuals with T2DM vs. NGM [0.152 SD $(0.007 ; 0.297)]$, an increase that was not clearly linear across deteriorating GM S (supplementary table S3.1).

Similar results were found when we investigated the linear associations of fasting glucose and $\mathrm{HbAlc}$ with plasma AGEs (supplementary table S3.2), or when we analysed the Hoorn and CODAM study separately (supplementary table S3.3).

\section{Associations of plasma AGEs with CVD}

Crude levels of protein-bound CEL and pentosidine and all free AGEs were significantly higher, whereas levels of protein-bound CML were significantly lower in individuals with compared to those without CVD (table 3.1).

However, after full adjustment for potential confounders (table 3.2), none of the proteinbound and free AGEs were significantly associated with prevalent CVD.

In analyses stratified according to GMS, we only found a significant positive association of protein-bound CEL with CVD in individuals with IGM [OR=1.37 (95\% Cl: 1.04; 1.81)], but an inverse association between this AGE and CVD in individuals with T2DM [OR=0.79 (0.63; 0.99)] (table 3.2). We found similar results when we analysed the Hoorn and CODAM cohorts separately (supplementary table S3.4). 


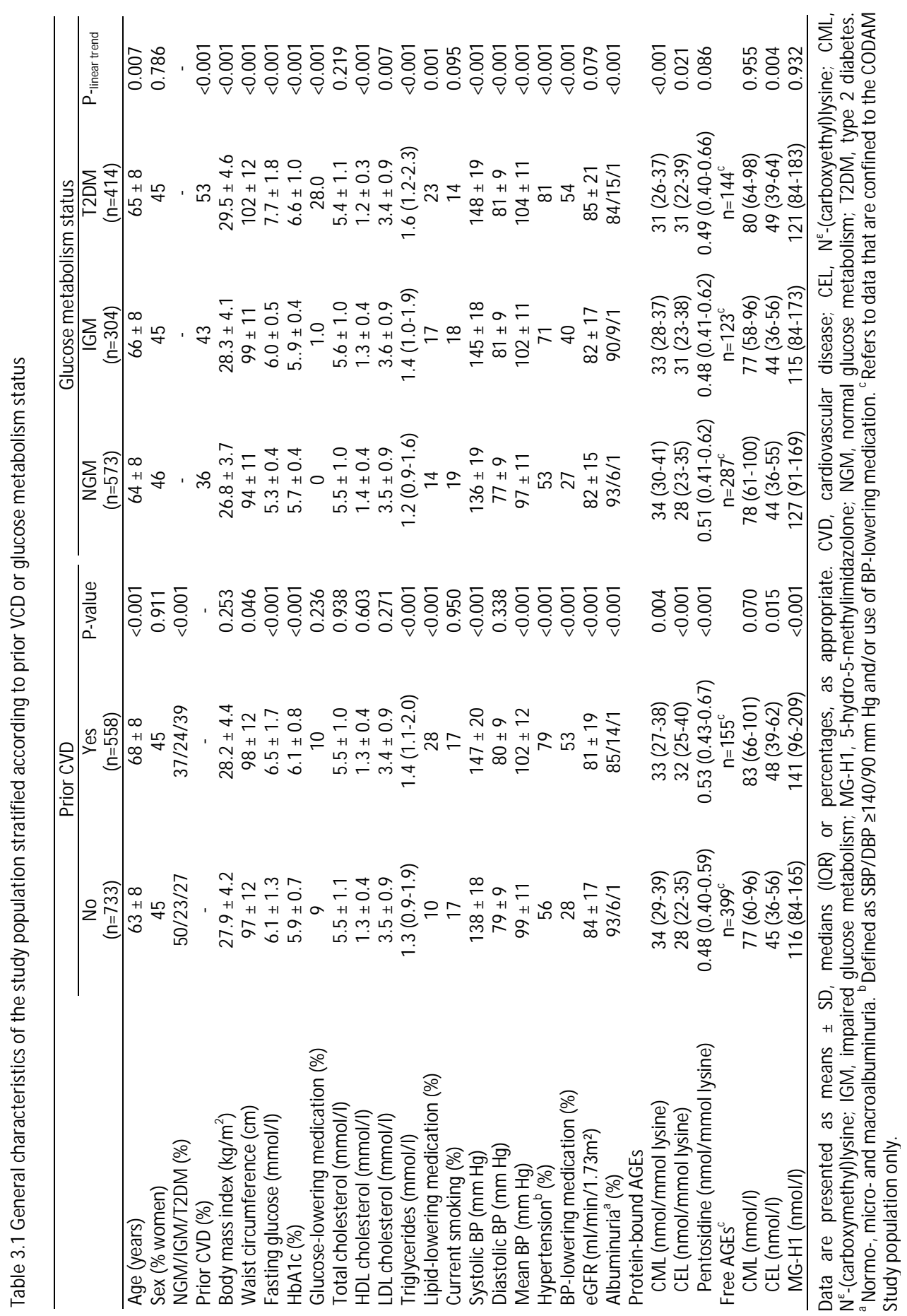




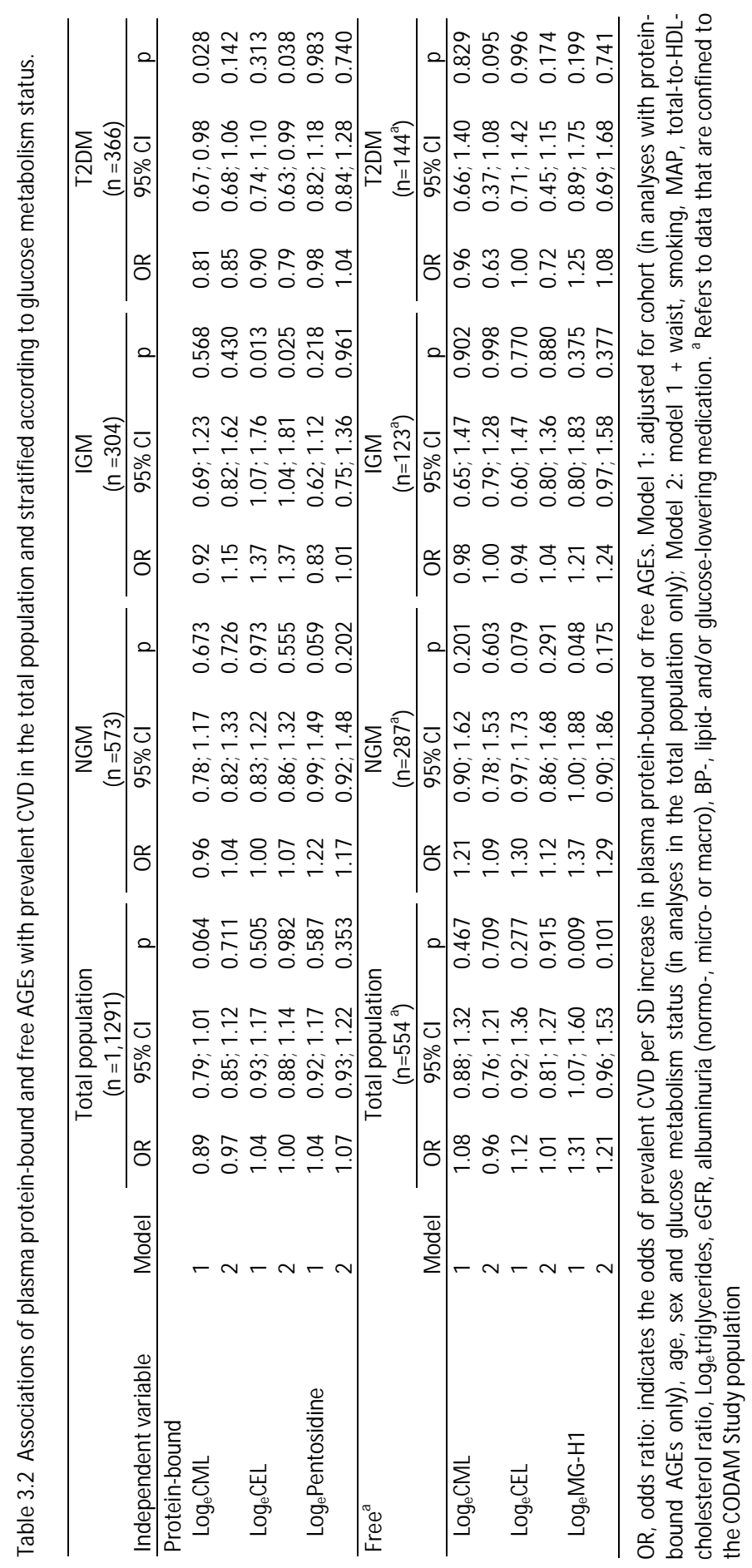




\section{Discussion}

This study had two main findings. First, after adjustments for potential confounders, plasma levels of protein-bound and free CML and CEL and free M G-H1 were not increased among individuals with deteriorating GMS. Only levels of protein-bound pentosidine were increased among individuals with T2DM only. Second, overall, none of the protein-bound and free AGEs were independently associated with prevalent CVD.

A general appraisal of the literature on associations between plasma AGES and CVD shows inconsistent findings, with either positive ${ }^{2-7}$ or null findings ${ }^{8-10}$. The same holds true for $\mathrm{T}_{2} \mathrm{DM}{ }^{7,8,10}$. Differences between these studies may be attributable by varying degrees of glycaemic control, but the full range of $\mathrm{HbAlc}$ in our study was 4.7 to $11.3 \%$, and we found no interaction between diabetic individuals with either good ( $\mathrm{HbAlc}<7.5 \%)$ or poor ( $\mathrm{HbAlc}>7.5 \%)$ glycemic control (data not shown). In addition, these differences could be due to small sample sizes and/or inconsistent or incomplete consideration of potential confounding factors in previously performed studies. Our findings in a rather large cohort describe a lack of adverse independent associations between plasma AGEs and CVD after adjustment for potential confounding factors. Our study thus illustrates the importance of these adjustments in cohort studies, because drawing inferences from unadjusted data would have led to different and misleading conclusions: e.g. that individuals with CVD had significantly lower levels of $C M L$ and higher levels of all remaining AGEs investigated herein (unadjusted data, table 3.1). Our study also extends previous ones by investigating the consistency of the associations within strata of deteriorating GMS. For instance, we found that plasma protein-bound CEL was positively associated with CVD in individuals with IGM, but inversely associated with CVD in individuals with T2DM. These contrasting associations are difficult to conciliate and most likely reflect a chance finding.

Several biological factors might explain the apparent contrasting findings between our study and the experimental work that has supported a causal role of AGEs in the development of (diabetes-related) vascular complications. First, many factors, such as dietary intake of $\mathrm{AGEs}^{15}$, ageing ${ }^{16}$, renal function ${ }^{17}$ or use of lipid-lowering medication ${ }^{18}$ may influence the concentrations of AGEs in tissues and plasma. In addition, plasma levels of the measured AGEs in this study may not adequately represent tissue AGE accumulation, as intracellular glycation is believed to be the major local source of AGEs ${ }^{19}$ and not all AGEs may end up in the circulation.

To our knowledge, our study is one of the few large cohort studies investigating associations between several plasma AGEs and prevalent CVD. Despite the large sample size, the use of state-of-the-art methodology for assessment of a wide range of both protein-bound and free AGEs in plasma, and the adjustment for potential confounders, our study has some limitations. Firstly, the study population consists of a middle-aged Caucasian population at high risk for (or with prevalent) T2DM and CVD. Our findings may thus not apply to the general Caucasian population or to other ethnicities. 
Secondly, given the cross-sectional design of our study, we cannot rule out the involvement of plasma AGEs in the development of incident CVD. Further (prospective) studies are warranted to fully address this issue. Indeed, we have shown that higher levels of protein-bound AGEs were associated with higher risk for incident CVD among individuals with type 1 diabetes ${ }^{20}$.

In conclusion, plasma AGEs are not associated with prevalent CVD in two large cohorts of individuals with NGM, IGM and T2DM. Although experimental studies underlined the importance of the AGEs measured in this study in the pathogenesis of CVD, our study suggests that the use of plasma levels of these AGEs, as biomarkers for increased CVD risk, may be limited in a population-based setting. Therefore, alternative measurements of AGE-burden, such as methylglyoxal, AGEs in circulating cells, urine or tissue may better reflect the AGE production in tissues and need to be further investigated in large cohort studies. 


\section{References}

1

2

Brownlee, M. Biochemistry and molecular cell biology of diabetic complications. Nature 414, 813-820 (2001).

2

Dworacka, M., Winiarska, H., Szymanska, M., Szczawinska, K. \& Wierusz-Wysocka, B. Serum Nepsilon-(carboxymethyl)lysine is elevated in nondiabetic coronary heart disease patients. Journal of basic and clinical physiology and pharmacology 13, 201-213 (2002).

Sugiyama, S. et al. Plasma levels of pentosidine in diabetic patients: an advanced glycation end product. J Am Soc Nephrol 9, 1681-1688 (1998).

Yoshida, N., Okumura, K. \& Aso, Y. High serum pentosidine concentrations are associated with increased arterial stiffness and thickness in patients with type 2 diabetes. M etabolism 54, 345-350 (2005).

Takahashi, R. et al. High serum concentrations of pentosidine, an advanced glycation end product, are associated with low normal value of ankle-brachial index in apparently healthy men. M etabolism 60, 649-654, doi:10.1016/j.metabol.2010.06.015 (2011).

Semba, R. D. et al. Advanced glycation end products and their circulating receptors predict cardiovascular disease mortality in older community-dwelling women. Aging Clin Exp Res 21, 182-190 (2009).

Semba, R. D., Bandinelli, S., Sun, K., Guralnik, J. M. \& Ferrucci, L. Plasma carboxymethyl-lysine, an advanced glycation end product, and all-cause and cardiovascular disease mortality in older community-dwelling adults. J Am Geriatr Soc 57, 1874-1880, doi:JGS2438 [pii] 10.1111/j.15325415.2009.02438.x (2009).

Kilhovd, B. K., Berg, T. J., Birkeland, K. I., Thorsby, P. \& Hanssen, K. F. Serum levels of advanced glycation end products are increased in patients with type 2 diabetes and coronary heart disease. Diabetes Care 22, 1543-1548 (1999).

Baumann, M. et al. Association between carotid diameter and the advanced glycation end product Nepsilon-carboxymethyllysine (CML). Cardiovasc Diabetol 8, 45, doi:10.1186/1475-2840-8-45 (2009).

Busch, $M$. et al. The advanced glycation end product $N$ (epsilon)-carboxymethyllysine is not a predictor of cardiovascular events and renal outcomes in patients with type 2 diabetic kidney disease and hypertension. Am J Kidney Dis 48, 571-579, doi:10.1053/j.ajkd.2006.07.009 (2006).

1 Scheijen, J. L., van de Waarenburg, M. P., Stehouwer, C. D. \& Schalkwijk, C. G. Measurement of pentosidine in human plasma protein by a single-column high-performance liquid chromatography method with fluorescence detection. Journal of chromatography. B, Analytical technologies in the biomedical and life sciences 877, 610-614, doi:10.1016/j.jchromb.2009.01.022 (2009).

2 Jacobs, $M$. et al. Low-grade inflammation can partly explain the association between the metabolic syndrome and either coronary artery disease or severity of peripheral arterial disease: the CODAM study. Eur J Clin Invest 39, 437-444 (2009).

Henry, R. M. et al. Arterial stiffness increases with deteriorating glucose tolerance status: the Hoorn Study. Circulation 107, 2089-2095 (2003).

Levey, A. S. et al. A more accurate method to estimate glomerular filtration rate from serum creatinine: a new prediction equation. Modification of Diet in Renal Disease Study Group. Ann Intern Med 130, 461-470, doi:199903160-00002 [pii] (1999). 
15 Koschinsky, T. et al. Orally absorbed reactive glycation products (glycotoxins): an environmental risk factor in diabetic nephropathy. Proc Natl Acad Sci U SA 94, 6474-6479 (1997).

16 Uribarri, J. et al. Circulating glycotoxins and dietary advanced glycation endproducts: two links to inflammatory response, oxidative stress, and aging. J Gerontol A Biol Sci M ed Sci 62, 427-433 (2007).

17 Lieuw-A-Fa, M. L. et al. Increased levels of N(epsilon)-(carboxymethyl)lysine and N(epsilon)(carboxyethyl)lysine in type 1 diabetic patients with impaired renal function: correlation with markers of endothelial dysfunction. Nephrol Dial Transplant 19, 631-636 (2004).

18 Cuccurullo, $C$. et al. Suppression of RAGE as a basis of simvastatin-dependent plaque stabilization in type 2 diabetes. Arterioscler Thromb Vasc Biol 26, 2716-2723, doi:10.1161/01.ATV.0000249630.02085.12 (2006).

19 Shinohara, M. et al. Overexpression of glyoxalase-I in bovine endothelial cells inhibits intracellular advanced glycation endproduct formation and prevents hyperglycemia-induced increases in macromolecular endocytosis. J Clin Invest 101, 1142-1147, doi:10.1172/JCl119885 (1998). Nin, J. W. et al. Higher plasma levels of advanced glycation end products are associated with incident cardiovascular disease and all-cause mortality in type 1 diabetes: a 12-year follow-up study. Diabetes Care 34, 442-447, doi:34/2/442 [pii] 10.2337/dc10-1087. 


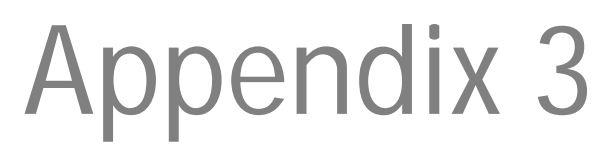

Supplemental methods and tables 


\section{Supplementary methods}

\section{Study population}

The present study included participants from the baseline examination of the Cohort on Diabetes and Atherosclerosis Maastricht (CODAM) Study ${ }^{1}$ and from the follow-up examination of the Hoorn Study ${ }^{2}$, these studies were designed to allow future pooling. Briefly, the CODAM study, which started in 1999-2000, is an ongoing prospective cohort study designed to investigate the effects of obesity, glucose and lipid metabolism, lifestyle and genetic factors on CVD. In total, 574 individuals were selected from a large population-based cohort based on an elevated risk for T2DM and/or CVD ${ }^{1}$. The initial Hoorn study started in 1989 as a population-based cohort study investigating glucose metabolism status (GMS) and CVD risk factors among a sample of the general population of Hoorn, the Netherlands. In 2000-2001, a follow-up examination was conducted, to which the current analyses were confined, in a selected group of 822 individuals consisting of a random sample of individuals with NGM and IGM and all individuals with T2DM of the follow-up cohort and 188 additional individuals with T2DM from the Hoorn Screening study?

Individuals missing data on plasma AGEs ( $n=88$ ) or CVD status $(n=17)$ were excluded from present analyses, resulting in a total of 1,291 individuals (554 from CODAM and 737 from Hoorn). Excluded individuals more often had T2DM and on average somewhat higher BMI, waist circumference, BP and lower HDL-cholesterol levels (data not shown).

Written informed consent was obtained from all participants and both cohort studies were approved by the local Medical Ethics Committees.

\section{M easurement of plasma AGEs}

Plasma AGEs were measured in EDTA plasma samples, obtained from fasting venous blood, which were stored at $-80^{\circ} \mathrm{C}$ until analysis. We developed and validated a new method to determine protein-bound CML and CEL and free CML, CEL and MG-H1 in plasma, using UPLC-tandem MS, which we describe in chapter $\mathbf{2 B}$.

Protein-bound pentosidine was measured using HPLC with a fluorescent detector, as described in chapter $2 \mathrm{~A}^{3}$. Concentrations of protein-bound $C M L, C E L$ and pentosidine were adjusted for levels of lysine and expressed as $\mathrm{nmol} / \mathrm{mmol}$ lysine. 


\section{Definition of glucose metabolism status}

In both cohorts participants' glucose metabolism status (GMS) was ascertained on the basis of fasting and 2-hour post-load glucose levels according to the WHO's criteria of 1999, as described in detail elsewhere ${ }^{1,2}$. In short, individuals with normal fasting $(\varangle 6.1 \mathrm{mmol} / \mathrm{L}$ ) and 2-hour post-load $(<7.8 \mathrm{mmol} / \mathrm{L})$ glucose levels were classified as having NGM. Individuals with impaired fasting glucose $(6.1-7.0 \mathrm{mmol} / \mathrm{L})$, impaired glucose tolerance (post-load glucose $7.8-11.1 \mathrm{mmol} / \mathrm{L}$ ) or both were classified as having IGM. Individuals using glucose lowering medication, insulin, and/or with a fasting plasma glucose of $\geq 7.0 \mathrm{mmol} / \mathrm{L}$ and/or 2 -hour post-load glucose of $\geq 11.1 \mathrm{mmol} / \mathrm{L}$ were classified as having T2DM. In total, 573 individuals had NGM, 304 had IGM and 414 had T2DM $(n=303$ newly identified and $n=111$ with known T2DM ).

\section{Definition of cardiovascular disease}

Prevalent CVD was defined as history of myocardial infarction, stroke or transient ischemic attack, non-traumatic limb amputation, coronary or peripheral artery bypass, angioplasty (self-reported), and/or signs of infarction or ischemia measured on a 12-lead ECG (M innesota codes 1-1 to 1-3, 4-1 to 4-3, 5-1 to 5-3 or 7-1) or an ankle brachial index of $\varangle .9$ in either leg ${ }^{1,2}$. Taken together, of the individuals with CVD, 83\% had coronary heart disease, $20 \%$ had cerebrovascular disease and $15 \%$ had peripheral arterial disease.

\section{Covariates}

Study covariates included waist circumference, smoking status, mean BP (M BP; calculated as $2^{*} \mathrm{DBP}+\mathrm{SBP}$ )/3), total, $\mathrm{HDL}$ and LDL cholesterol, triglycerides, estimated (e) GFR (using the short Modification of Diet in Renal Disease equation ${ }^{4}$ ), presence of micro- or macroalbuminuria and use of BP-, lipid- and glucose-lowering medication, and were determined as described earlier ${ }^{1,2}$.

\section{Statistical analyses}

All statistical analyses were carried out using the Predictive Analytics SoftWare, version 18.0 (SPSS IBM Corporation, Armonk, NY, USA). Skewed variables (i.e. CML, CEL, pentosidine, M G-H1, triglycerides) were $\log _{\mathrm{e}}$ transformed prior to all analyses. 
Imputation of missing covariate data

A total of 88 individuals ( $7 \%$ of the total population) had missing values for one $(n=82)$ or more $(n=6)$ of the covariates. The percentage of missing values per variable varied from $0.1 \%$ (current smoking) to $3.5 \%$ (BP). We used multiple imputation chained equations to impute those values rather than perform complete case analyses to decrease selection bias and increase power of the analyses ${ }^{5}$. Results reported were those retrieved from pooled analyses on all five imputed datasets.

\section{Data analyses}

General characteristics of the combined CODAM and Hoorn study populations were compared between individuals with and without prior CVD and across levels of GMS with the use of ANOVA or $\chi^{2}$-tests, for continuous or categorical data, respectively.

We used linear regression analyses to compare levels of AGEs between individuals with IGM or T2DM vs. NGM (reference group), first, after adjustments for age, sex, and cohort (model 1) and additionally for waist, smoking, MAP, blood lipids, eGFR, albuminuria and use of medication (model 2) to fully rule out potential confounding by these factors. We used logistic regression analyses to investigate the associations between plasma AGEs and prevalent CVD. These analyses were conducted in the whole study population and stratified according to individuals' GMS, and included adjustments for covariates as described in models 1 and 2 above. Results of these analyses are expressed in standardized regression coefficients.

We also tested whether associations differed between cohorts or between men and women by adding interaction terms to our models, but did not find any such consistent interactions ( $p$-values for interaction were $>0.1$ ). Among the individuals with diabetes, the same was true for individuals with newly identified- and with known T2DM, and individuals with a low $(<7.5 \%)$ or high $(\geq 7.5 \%) \mathrm{HbAlc}$. Therefore, all results are shown without stratification for these factors. 


\section{References online-methods}

1 Jacobs, M. et al. Low-grade inflammation can partly explain the association between the metabolic syndrome and either coronary artery disease or severity of peripheral arterial disease: the CODAM study. Eur J Clin Invest 39, 437-444 (2009).

2 Henry, R. M. et al. Arterial stiffness increases with deteriorating glucose tolerance status: the Hoorn Study. Circulation 107, 2089-2095 (2003).

3 Scheijen, J. L., van de Waarenburg, M. P., Stehouwer, C. D. \& Schalkwijk, C. G. Measurement of pentosidine in human plasma protein by a single-column high-performance liquid chromatography method with fluorescence detection. J Chromatogr B Analyt Technol Biomed Life Sci 877, 610-614, doi:S1570-0232(09)00043-9 [pii] 10.1016/j.jchromb.2009.01.022 (2009).

4 Levey, A. S. et al. A more accurate method to estimate glomerular filtration rate from serum creatinine: a new prediction equation. Modification of Diet in Renal Disease Study Group. Ann Intern Med 130, 461-470, doi:199903160-00002 [pii] (1999).

5 Janssen, K. J. et al. Missing covariate data in medical research: to impute is better than to ignore. Journal of clinical epidemiology 63, 721-727, doi:10.1016/j.jclinepi.2009.12.008 (2010). 


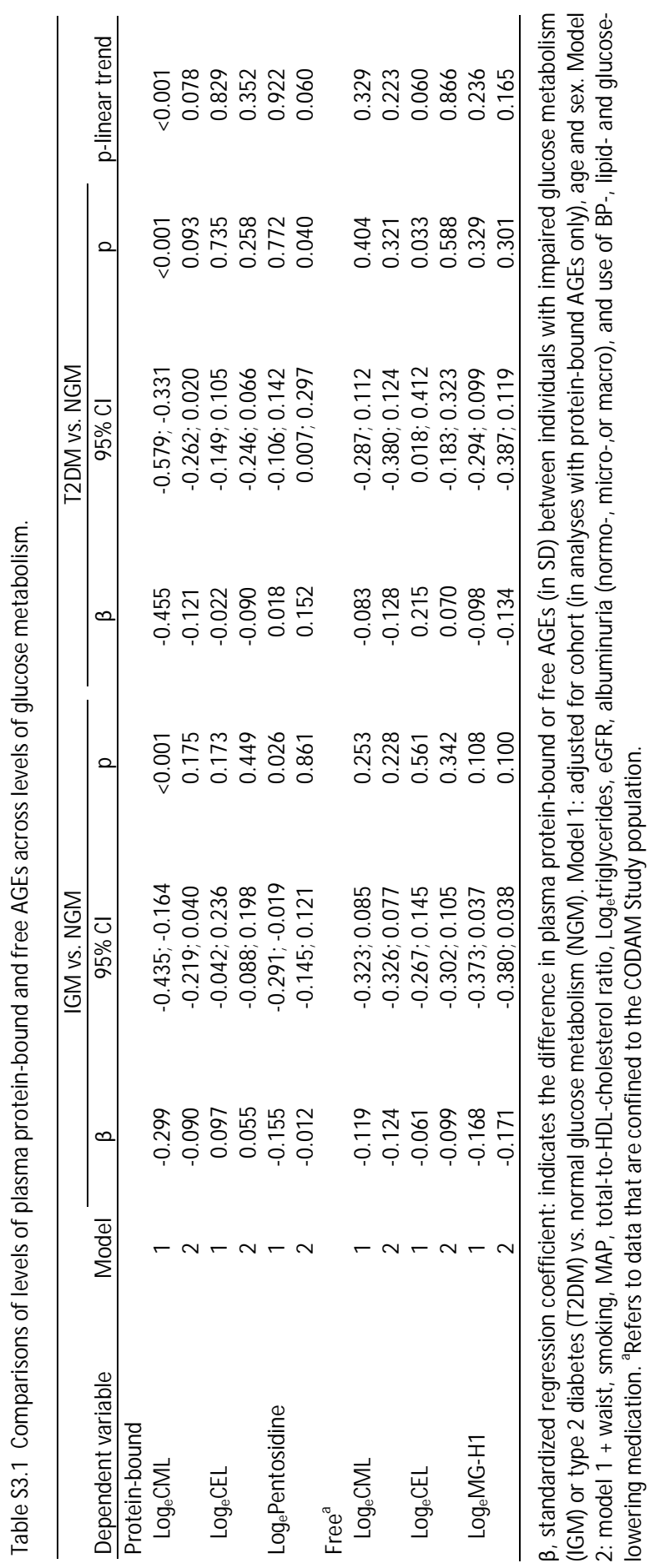




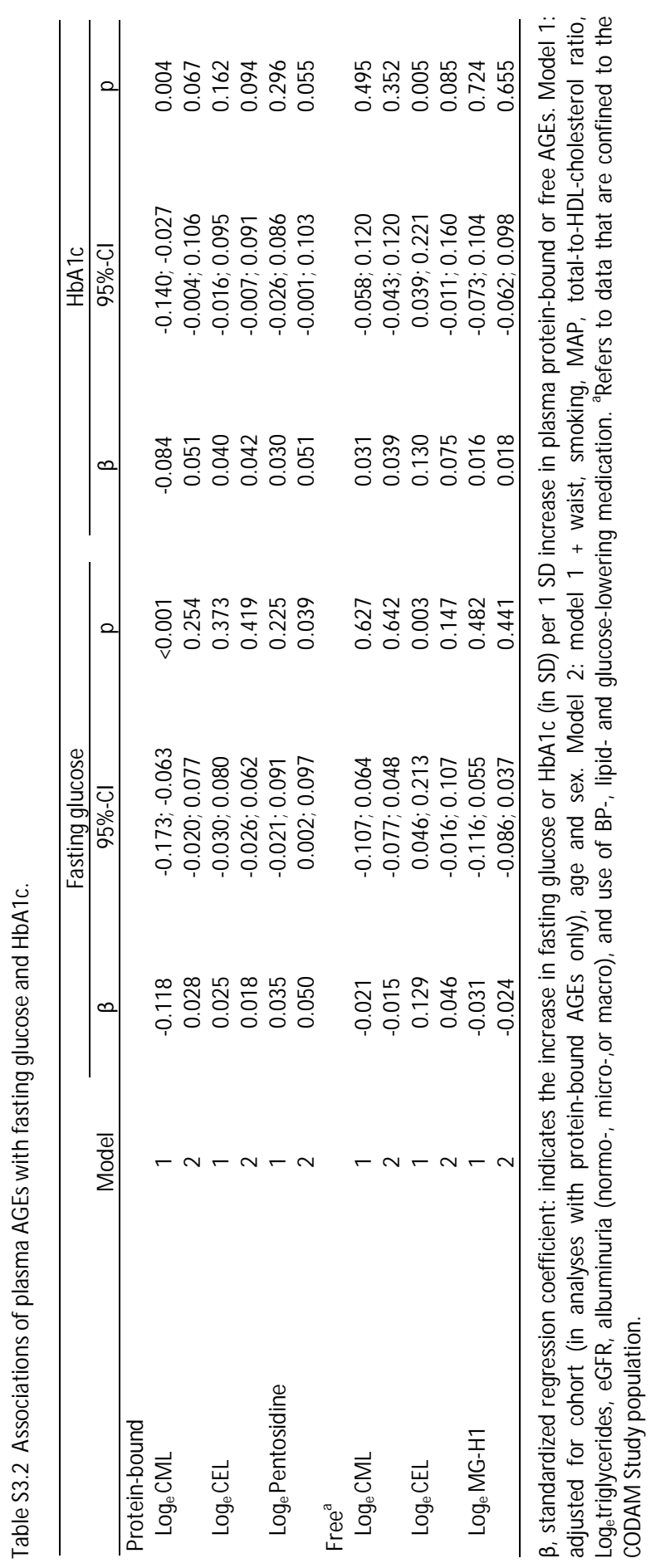




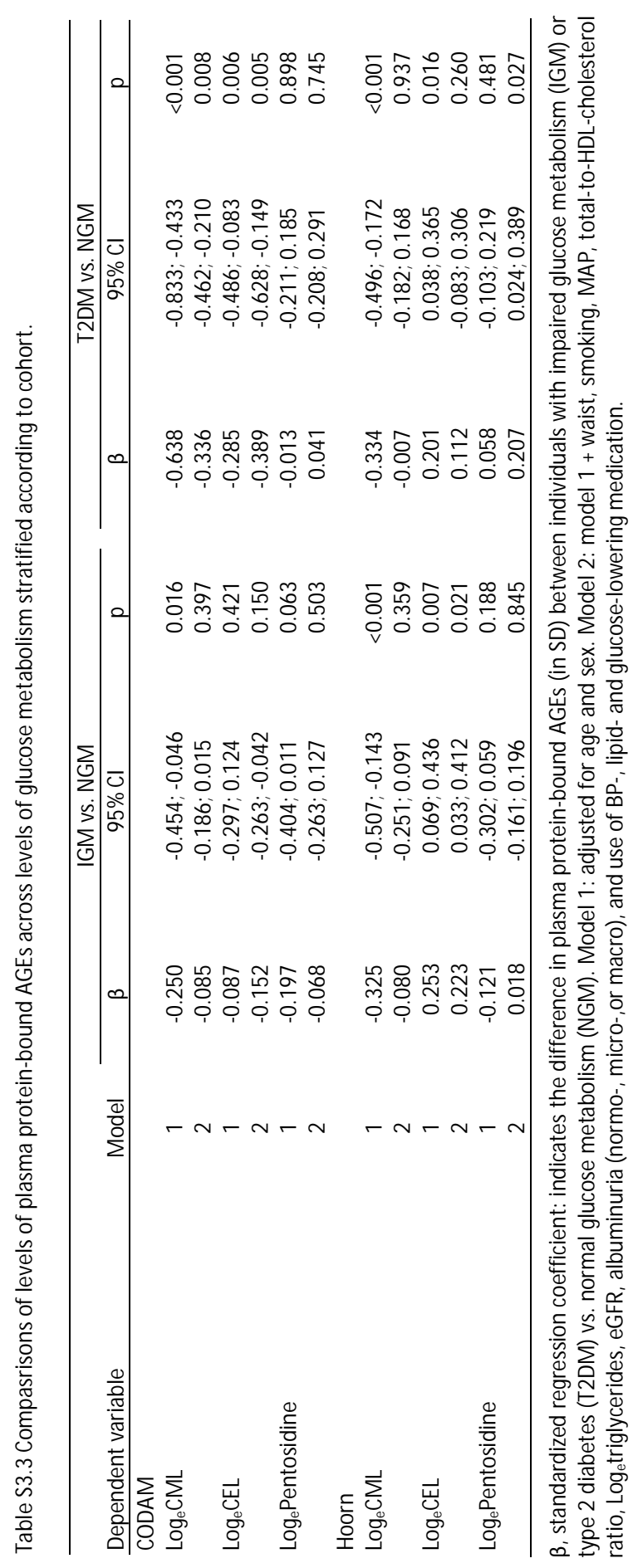


Table S3.4 Associations of plasma AGEs with prevalent CVD stratified according to cohort.

\begin{tabular}{|c|c|c|c|c|}
\hline \multirow[b]{2}{*}{ Independent variable } & \multirow[b]{2}{*}{ Model } & \multicolumn{3}{|l|}{ CVD } \\
\hline & & $\mathrm{OR}$ & $95 \% \mathrm{Cl}$ & $p$ \\
\hline \multicolumn{5}{|l|}{ CODAM } \\
\hline \multirow[t]{2}{*}{$\log _{e} C M L$} & 1 & 0.77 & $0.62 ; 0.95$ & 0.013 \\
\hline & 2 & 0.91 & $0.81 ; 1.03$ & 0.453 \\
\hline \multirow[t]{2}{*}{$\log _{\mathrm{e}} \mathrm{CEL}$} & 1 & 0.99 & $0.82 ; 1.21$ & 0.942 \\
\hline & 2 & 0.95 & $0.85 ; 1.06$ & 0.654 \\
\hline \multirow[t]{2}{*}{$\log _{\mathrm{e}}$ Pentosidine } & 1 & 0.87 & $0.71 ; 1.06$ & 0.173 \\
\hline & 2 & 0.94 & $0.75 ; 1.18$ & 0.597 \\
\hline \multicolumn{5}{|l|}{ Hoorn } \\
\hline \multirow[t]{2}{*}{$\log _{e} C M L$} & 1 & 0.96 & $0.82 ; 1.12$ & 0.576 \\
\hline & 2 & 1.00 & $0.84 ; 1.20$ & 0.970 \\
\hline \multirow[t]{2}{*}{$\log _{\mathrm{e}} \mathrm{CEL}$} & 1 & 1.07 & $0.92 ; 1.24$ & 0.397 \\
\hline & 2 & 1.03 & $0.88 ; 1.22$ & 0.697 \\
\hline \multirow[t]{2}{*}{ Log $_{\mathrm{e}}$ Pentosidine } & 1 & 1.16 & $0.98 ; 1.37$ & 0.085 \\
\hline & 2 & 1.17 & $0.98 ; 1.41$ & 0.086 \\
\hline
\end{tabular}

$\mathrm{OR}$, odds ratio: indicates the odds of prevalent CVD per SD increase in plasma protein-bound AGEs. Model 1: adjusted for age, sex and glucose metabolism status.

Model 2: model 1 + waist, smoking, MAP, total-to-HDL-cholesterol ratio, Logetriglycerides, eGFR, albuminuria (normo-, micro-,or macro), and use of BP-, lipid- and glucose-lowering medication. 



\section{$L(+)$ and $D(-)$ Lactate}

ARE INCREASED IN PLASMA AND URIN S SAMPLES OF TY PE 2 DIABEIIES AS MEASUR DD BY A SIMULTANEOUS QUANIIFCAIIONOF

$L(+)$ and D(-) Lactate

BY REVERSED PHASE HCUID CHROMATCERADHY Tandem Mass Spectrometry 


\section{4}

$L(+)$ and $D(-)$ Lactate are increased in plasma and urine samples of type 2 diabetes as measured by a simultaneous quantification of $L(+)$ and D(-) Lactate by Reversed Phase Liquid Chromatography Tandem Mass Spectrometry

Jean LJ.M. Scheijen, Nordin M.J. Hanssen, M arjo P.H. van de Waarenburg, Daisy M.A.EJ Jonkers, Coen D.A. Stehouwer and Casper G. Schalkwijk

Exp Diabetes Res. 2012; 1-10 (2012) 


\section{Abstract}

\section{Background:}

Plasma and urinary levels of D-lactate have been linked to the presence of diabetes. Previously developed techniques have shown several limitations to further evaluate D-lactate as a biomarker for this condition.

\section{Methods:}

D- and L-lactate were quantified using ultra performance liquid chromatography tandem mass spectrometry with labelled internal standard. Samples were derivatized with diacetyl-L-tartaric anhydride and separated on a C18-reversed phase column. D- and L-lactate were analysed in plasma and urine of controls, patients with inflammatory bowel disease (IBD) and patients with type 2 diabetes (T2DM ).

\section{Results:}

Quantitative analysis of D- and L-lactate was achieved successfully. Calibration curves were linear ( $r 2>0.99$ ) over the physiological and pathophysiological range. Recoveries for urine and plasma were between $96 \%$ and $113 \%$. Inter- and intra-assay variations were between $2 \%$ and $9 \%$. The limits of detection of D-lactate and L-lactate in plasma were $0.7 \mu \mathrm{mol} / \mathrm{L}$ and $0.2 \mu \mathrm{mol} / \mathrm{L}$ respectively. The limits of detection of D-lactate and L-lactate in urine were $8.1 \mathrm{nmol} / \mathrm{mmol}$ creatinine and $4.4 \mathrm{nmol} / \mathrm{mmol}$ creatinine, respectively. Plasma and urinary levels of D- and L-lactate were increased in patients with IBD and T2DM as compared with controls.

\section{Conclusion:}

The presented method proved to be suitable for the quantification of D- and L-lactate and opens the possibility to explore the use of D-lactate as a biomarker. 


\section{Introduction}

There are several conditions in which D-lactate can become increased in blood and urine in humans ${ }^{1}$. Recent studies demonstrated increased levels of D-lactate in diabetes and in infection, ischemia and trauma, suggesting the use of D-lactate as a biomarker. However, to further explore the use of D-lactate as such a biomarker there is a need of an improved method for analysing D- lactate.

Lactate has two optical isomers, L-lactate and D-lactate (figure 4.1b). L-lactate is the most abundant enantiomer of lactate. It is formed mainly during anaerobic glycolysis by conversion of pyruvate to L-lactate by Lactate dehydrogenase ${ }^{2}$. D-lactate is often considered as the non-physiological counterpart of L-lactate ${ }^{1}$. Under physiologic conditions the concentration of D-lactate is a 100-fold lower when compared to L-lactate ${ }^{3}$. The origin of D-lactate in human metabolism is thought to be derived from two major sources, namely degradation of methylglyoxal into D-lactate by the glyoxalase pathway and production by intestinal bacteria. Indeed, disturbances in these metabolic pathways are associated with increased levels of D-lactate ${ }^{3-7}$. Although some enzymes capable of metabolizing $D$-lactate have been described ${ }^{8}$, its metabolism is very inefficient and D-lactate is mainly excreted in urine'.

Methylglyoxal is a highly reactive compound formed in the process of glycolysis and lipid peroxidation. Methylglyoxal is increased in diabetes and is a major precursor in the formation of advanced glycation endproducts ${ }^{9}$. Methylglyoxal is degraded by the glyoxalase system resulting in D-lactate. D-lactate in plasma and urine has been shown to be increased in patients with diabetes ${ }^{3,7}$. D-lactate can be used as a reflection of methylglyoxal and is much easier to measure than the very reactive methylglyoxal.

In the colon, many commensal bacteria produce D-lactate as a result of anaerobic glycolysis. Under physiological circumstances this D-lactate is further metabolized by the commensal bacteria to acetate. Therefore, D-lactate produced in the intestinal tract does not significantly contribute to levels of D-lactate in the systemic circulation under physiological circumstances ${ }^{10}$. However, under pathologic conditions systemic D-lactate levels may rise due to intestinal production by bacteria. In patients with ulcerative colitis, gut ischemia and appendicitis, increased levels of D-lactate have indeed been demonstrated $^{4-6}$. The most extreme example of impaired gut permeability and bacterial overgrowth is short bowel syndrome, which is associated with D-lactate acidosis ${ }^{10}$.

So far, D- and L-lactate have been analysed by several different techniques ranging from chiral stationary phase liquid chromatography using UV or fluorescence detection ${ }^{3,11-15}$, enzymatic assays ${ }^{7,16-21}$, gas chromatography mass spectrometry (GC/MS) methods ${ }^{22,23}$, liquid chromatography mass spectrometry (LC/MS) methods ${ }^{24,25}$ and reversed phase liquid chromatography using fluorescence detection ${ }^{26}$. However, these techniques have several shortcomings such as low sensitivity ${ }^{11,24,27}$ and large sample volume ${ }^{19,21,22}$, complex chromatographic systems $s^{3,12,13}$ and long run times $3,11,26,27$. To further explore the use of D-lactate as a biomarker there is a need of an improved method for analysing D-lactate. 
In this paper we describe a highly sensitive, specific and fast ultra performance liquid chromatography (UPLC) tandem mass spectrometry (MS/MS) method for the analysis of $\mathrm{D}$ - and L-lactate in plasma and urine without the need of a chiral stationary phase. We achieved a significant improvement over the methods described in the literature and obtained a strong tool for the analysis of D- and L-lactate in large studies. With this method we measured plasma and urine concentrations of D- and L-lactate in controls, patients with inflammatory bowel disease (IBD) and in patients with type 2 diabetes (T2DM).

\section{Materials and methods}

\section{Chemicals and reagents}

$\mathrm{L}(+)$-Lactate $(98 \%)$ and Dichloromethane $(\geq 99.9 \%)$ were obtained from Sigma-Aldrich. Ammonia solution (25\%) and Acetic acid anhydrous (100\%) were obtained from Merck. Formic acid (р.а.), (+)-0,0'-diacetyl-L-tartaric anhydride ( $\geq 97 \%$ ) (DATAN) and lithium D-lactate ( $\geq 99 \%)$ were obtained from Fluka. Water and acetonitril (ULC-M S grade) were obtained from Biosolve. [13C -3 ]-Sodium L-lactate (20\%, w/w in water) was obtained from Cambridge Isotope Laboratories.

\section{Chromatographic conditions}

Samples were analysed by reversed phase LC-tandem MS using an Acquity UPLC BEH C18 analytical column ( $100 \times 2.1 \mathrm{~mm}, 1.7 \mu \mathrm{m}$, Waters). Detection was carried out using a Xevo TQ tandem mass spectrometer (Waters), which was operated in negative multiplereaction-monitoring (MRM) mode. UPLC analysis was performed using a binary gradient at a flow of $0.5 \mathrm{ml} / \mathrm{min}$ using an Acquity UPLC (Waters). Solvent A was $1.5 \mathrm{mM}$ ammonium formate $(\mathrm{pH}=3.6)$ and solvent $B$ was acetonitril. A linear gradient was started at $99.5 \%$ solvent $A$, which was changed within 3 minutes to $97 \%$ solvent $A$. After cleaning the column with $40 \%$ solvent B during 2 minutes the column was equilibrated for 1 minute at the initial composition. Injection volume was $2 \mu \mathrm{l}$ and column temperature was set at $31^{\circ} \mathrm{C}$. Samples were kept at $6^{\circ} \mathrm{C}$. Chromatograms were acquired and processed with Masslynx V4.1 SCN 644 (Waters). 


\section{Mass spectrometry conditions}

MRM transitions were optimised using direct infusion of D-lactate $(500 \mu \mathrm{mol} / \mathrm{L})$, $\left[{ }^{13} \mathrm{C}_{3}\right]$-L-Lactate $(400 \mu \mathrm{mol} / \mathrm{L})$ and L-lactate $(1000 \mu \mathrm{mol} / \mathrm{L})$ standard solution into the tandem MS at a flow of $20 \mu \mathrm{l} / \mathrm{min}$. Optimal conditions for all parents were found at a capillary voltage of $1.5 \mathrm{kV}$ and a cone voltage of $10 \mathrm{~V}$. The source and desolvation temperature were 150 and $450^{\circ} \mathrm{C}$ respectively. The cone gas flow and desolvation gas flow were 0 and $800 \mathrm{l} /$ hour respectively. To establish the most sensitive daughter ions the collision energy was set at $8 \mathrm{eV}$ with a collision gas flow of $0.15 \mathrm{ml} / \mathrm{min}$. Table 4.1 shows the optimised MRM settings.

Table 4.1 M RM settings

\begin{tabular}{lllll}
\hline Component & Parent Ion $(\mathbf{m} / \mathbf{z})$ & Daughter Ion $(\mathrm{m} / \mathbf{z})$ & Collision energy $(\mathrm{eV})$ & Dwell $(\mathrm{secs})$ \\
\hline$\left[{ }^{13} \mathrm{C}_{3}\right]$-L-Lactate & 307.95 & 91.95 & 8.0 & 0.1 \\
D-Lactate & 304.95 & 88.95 & 8.0 & 0.1 \\
L-Lactate & 304.95 & 88.95 & 8.0 & 0.1 \\
\hline
\end{tabular}

\section{Plasma and Urine samples}

Three groups were selected for D- and L-lactate measurements. Diabetic individuals and non-diabetic controls were sex- and age matched subsets recruited from the Cohort study of Diabetes and Atherosclerosis Maastricht (CODAM). The characteristics of these subjects have been described in detail elsewhere [28]. In short, the control group $(n=52)$ was $55.8 \pm 0.7$ years old, $46 \%$ female and had a $\mathrm{HbAlC}$ of $5.6 \pm 0.1 \%$ and a fasting plasma glucose of $5.2 \pm 0.1 \mathrm{mmol} / \mathrm{L}$. Group 2, the patients with T2DM $(n=52)$, were $56.3 \pm 0.6$ years old, $39 \%$ female and had a $\mathrm{HbAlC}$ of $6.9 \pm 0.2 \%$ and fasting plasma glucose of $8.0 \pm 0.2 \mathrm{mmol} / \mathrm{L}$. Group 3 consisted of patients with IBD in remission; 32 plasma samples (52.3 \pm 8.6 years, $44 \%$ female) and 34 urine samples (54.6 \pm 14.1 years, $59 \%$ female). These samples were recruited from the out-patient-clinic of the Maastricht University Medical Center.

For comparison of the proposed UPLC tandem MS method with the enzymatic method, we analysed plasma and urinary D-lactate, with both methods, in rat samples. These animals were described in details elsewhere ${ }^{29}$.

\section{Plasma sample preparation}

To $25 \mu$ of internal standard solution (containing $434.75 \mu \mathrm{mol} / \mathrm{L}\left[{ }^{13} \mathrm{C}_{3}\right]$-L-Lactate) $25 \mu \mathrm{l}$ of plasma was added. Samples were mixed thoroughly and subsequently deproteinized with $600 \mu \mathrm{l}$ of a mixture of methanol:acetonitril (1:1, by volume) and centrifuged at room temperature during 10 minutes at $14000 \mathrm{rpm}$. 
The supernatant was pipetted into a reaction vial and evaporated to dryness under a gentle stream of nitrogen at a temperature of $50^{\circ} \mathrm{C}$. Fifty micro liters of freshly made DATAN (50 mg/ $\mathrm{ml}$ dichloromethane:acetic acid (4:1, by volume)) was added. The vial was capped, vortexed and heated at $75^{\circ} \mathrm{C}$ for 30 minutes. After 30 minutes the vial was allowed to cool down to room temperature and the mixture was evaporated to dryness with a gentle stream of nitrogen. The derivatized residue was reconstituted with $150 \mu \mathrm{l}$ acetonitril:water (1:2, by volume).

\section{Urine sample preparation}

Twenty-five microliters of internal standard solution (containing $434.75 \mu \mathrm{mol} / \mathrm{L}$ $\left[{ }^{13} \mathrm{C}_{3}\right]$-L-Lactate), $25 \mu \mathrm{l}$ urine and $300 \mu$ of methanol was pipetted into a reaction vial. Samples were mixed thoroughly and evaporated to dryness under a gentle stream of nitrogen at a temperature of $50^{\circ} \mathrm{C}$. Fifty microliters of freshly made DATAN $(50 \mathrm{mg} / \mathrm{ml}$ dichloromethane:acetic acid (4:1, by volume)) was added. The vial was capped, vortexed and heated at $75^{\circ} \mathrm{C}$ for 30 minutes. After 30 minutes the vial was allowed to cool down to room temperature and the mixture was evaporated to dryness with a gentle stream of nitrogen. The derivatized residue was reconstituted with $300 \mu$ acetonitril:water (1:2, by volume).

\section{Method validation}

Linearity of the detection of D- and L-lactate was tested in water and matrix by adding Dand L-lactate standard to water and during preparation of plasma or urine samples (table 4.2). Calibration curves were obtained by linear regression of a plot of the analyte concentration $(x)$ vs the peak-area ratio of the analyte/internal standard area ( $y)$. For both the analytes, $\left[{ }^{13} \mathrm{C}_{3}\right]$-L-lactate was used as internal standard.

The lower limit of detection was determined by calculating the concentration at a signal to noise ratio of six ( $\mathrm{s} / \mathrm{N}: 6$, injection volume: $2 \mu \mathrm{l})$.

For recovery experiments, standard solutions of $\mathrm{D}$ - and L-lactate were added to urine or plasma and subsequently prepared as described in the sample preparation section.

The intra-assay variation of the method was determined in two different plasma and urine samples $(n=10)$ analysed in one batch during one day. The inter-assay variation of the method was determined in two different plasma and urine samples divided into batches and analysed during 10 different days.

Freeze-thaw stability was tested in two different plasma and urine samples by snapfreezing these samples in liquid nitrogen and thawing them for 5 subsequent cycles.

To investigate the stability of plasma and urine samples, stored at $6^{\circ} \mathrm{C}$ in the autosampler, replicate injections of two different plasma and urine samples were done every hour during 24 hours. 


\section{Determination of fasting plasma glucose, $\mathrm{HbalC}$ and urinary creatinine}

After an overnight fast, plasma glucose concentrations $(\mathrm{mmol} / \mathrm{L})$ were measured with a hexokinase glucose- 6 phosphate dehydrogenase method (ABX Diagnostics, M ontpellier, France). Hba1C (\%) was determined by ion-exchange high-performance liquid chromatography (HPLC) (Bio-Rad, Veenendaal, the Netherlands). Fasting plasma glucose concentrations and $\mathrm{Hba1C}$ were determined in the CODAM participants only.

Urinary D- and L-lactate concentrations were expressed as $\mu \mathrm{mol} / \mathrm{mmol}$ creatinine. Creatinine concentration in urine was analysed using a Beckman LX20 analyser (Beckman Coulter) based on the Jaffé reaction method ${ }^{28}$.

\section{D-lactate enzymatic assay}

For method comparison, an enzymatic-spectrophotometric method, based on the oxidation of D-lactate tot pyruvate by $N A D+$ in the presence of bacterial D-lactate hydrogenase was used ${ }^{21}$.

\section{Statistical Analysis}

The method validation data were expressed as mean and standard deviation (SD). To investigate agreement between the enzymatic and UPLC tandem MS method we used linear regression and a Bland-Altman plot after log normalisation of the rat urine samples. Limits of agreement were defined as 2 times the SD. The patient study data were expressed as mean and standard error of the mean (SEM). To detect group differences we applied analysis of variance (ANOVA) with post-hoc Bonferroni correction. P-value $\varangle 0.05$ was considered statistically significant.

\section{Results}

\section{Reversed phase chromatography}

D- and L-lactate DATAN derivatives yielded a baseline separation on a reversed phase UPLC column with a retention time of 2.7 minutes for D-lactate and 2.5 minutes for L-lactate. Representative chromatograms are shown in figure 4.1. 


\section{Stability of D- and L-lactate}

After 5 freeze-thaw cycles no change of D- and L-lactate levels was observed, as tested in two different plasma and urine samples (data not shown).

To make large number of measurements within one run possible, we tested the stability of D- and L-lactate when samples were stored in the auto-injector at $6^{\circ} \mathrm{C}$. D- and L-lactate were at least stable for 24 hours, no degradation was observed after replicate injections of two different plasma and urine samples (data not shown).
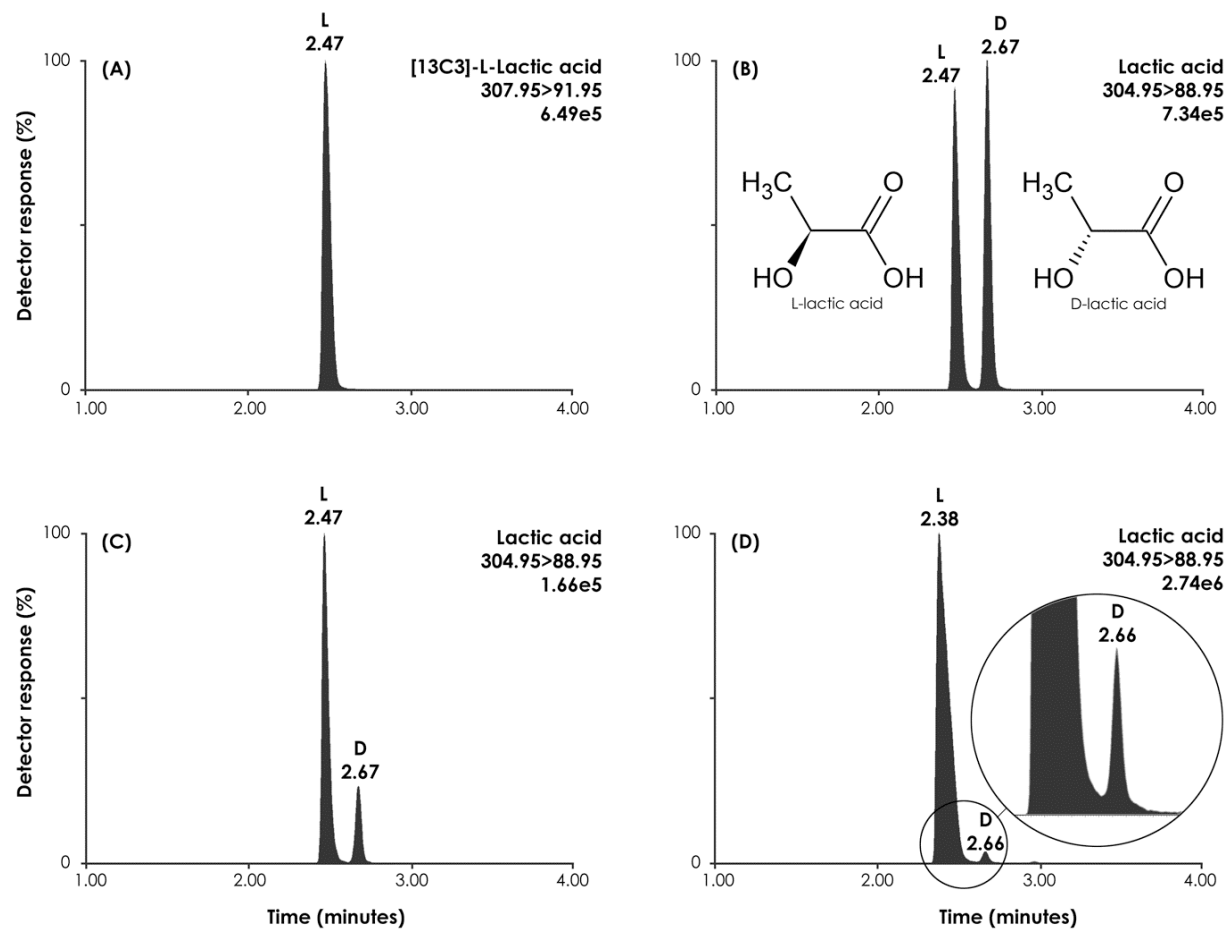

Figure 4.1. Representative chromatograms of D- and L-lactic acid derivatives ${ }^{(a)}$

(A) Internal standard [13C3]-L-lactate chromatogram (435 $\mu \mathrm{mol} / \mathrm{L}, 72 \mathrm{pmol})$.

(B) Representative chromatogram of a standard solution of D- and L-lactate $(351 \mu \mathrm{mol} / \mathrm{L}, 58.2 \mathrm{pmol}$ and 501 $\mu \mathrm{mol} / \mathrm{L}, 83.0 \mathrm{pmol}$ respectively) and molecular structures of optical isomers L- and D-lactate.

(C) Representative chromatogram of a urine sample (D- and L-lactate; $14.1 \mu \mathrm{mol} / \mathrm{L}, 2.3 \mathrm{pmol}$ and $74.6 \mu \mathrm{mol} / \mathrm{L}$, 12.4 pmol respectively).

(D) Representative chromatogram of a plasma sample (D- and L-lactate; $11.2 \mu \mathrm{mol} / \mathrm{L}, 1.9 \mathrm{pmol}$ and $1375.0 \mu \mathrm{mol} / \mathrm{L}, 227.7 \mathrm{pmol}$ respectively).

(a) Injection volume: $2 \mu \mathrm{l}$ 


\section{Linearity, lower limit of detection, recovery and precision}

Linearity of the detection of D- and L-lactate was tested in matrix and water. For plasma the slope, tested in three different plasma samples and in water measured on different days, was $1.4 \pm 6.3 \%$ (mean \pm CV\%) for D-lactate and $0.75 \pm 3.2 \%$ for L-lactate (table 4.2 ). For urine the slope, tested in three different urine samples and in water measured on different days, was $1.09 \pm 4.0 \%$ for D-lactate and $0.80 \pm 1.6 \%$ for L-lactate (table 4.2 ). The limits of detection of D-lactate and L-lactate in plasma were $0.65 \mu \mathrm{mol} / \mathrm{L}(108 \mathrm{fmol}$ ) and $0.2 \mu \mathrm{mol} / \mathrm{L}(33 \mathrm{fmol}$ ) respectively. The limits of detection of D-lactate and L-lactate in urine were $8.1 \mathrm{nmol} / \mathrm{mmol}$ creatinine $(40 \mathrm{fmol})$ and $4.4 \mathrm{nmol} / \mathrm{mmol}$ creatinine $(22 \mathrm{fmol})$ respectively. We found recoveries, for urine and plasma, between $96 \%$ and $113 \%$ (table 4.3). The validation data demonstrated inter- and intra-assay variations between $2 \%$ and $9 \%$ (table 4.4 ).

Table 4.2 Linearity tested in different matrices.

\begin{tabular}{lllll}
\hline $\begin{array}{l}\text { Matrix } \\
\text { D-lactate }\end{array}$ & Slope $^{(a)}$ & Y-intercept & $r^{2}$ & Concentration range $(\mu \mathrm{mol} / \mathbf{L})$ \\
\hline Water & 1.2927 & -4.5 & 0.9971 & $0-105$ \\
Plasma A & 1.4307 & 21.2 & 0.9987 & $0-105$ \\
Plasma B & 1.3820 & 51.3 & 0.9991 & $0-105$ \\
Plasma C & 1.5037 & 31.9 & 0.9990 & $0-105$ \\
Mean & 1.4023 & & & \\
CV(\%) & $\mathbf{6 . 3}$ & & & \\
\hline
\end{tabular}

\begin{tabular}{lllll}
\hline L-lactate & & & & \\
\hline Water & 0.7768 & -4.9 & 0.9996 & $0-3008$ \\
Plasma A & 0.7566 & 1032 & 0.9998 & $0-6016$ \\
Plasma B & 0.7164 & 996 & 0.9997 & $0-6016$ \\
Plasma C & 0.7534 & 1346 & 0.9997 & $0-3008$ \\
Mean & $\mathbf{0 . 7 5 0 2}$ & & & \\
CV(\%) & 3.2 & & & \\
\hline
\end{tabular}

\begin{tabular}{lllll}
\hline D-lactate & \multicolumn{5}{l}{} \\
\hline Water & 1.1265 & -15.4 & 0.9999 & $0-702$ \\
Urine A & 1.1092 & 105.24 & 0.9984 & $0-351$ \\
Urine B & 1.1114 & 99.7 & 0.9999 & $0-702$ \\
Urine C & 1.0287 & 69.8 & 0.9992 & $0-351$ \\
Mean & 1.094 & & & \\
CV(\%) & $\mathbf{4 . 0 4}$ & & & \\
\hline
\end{tabular}

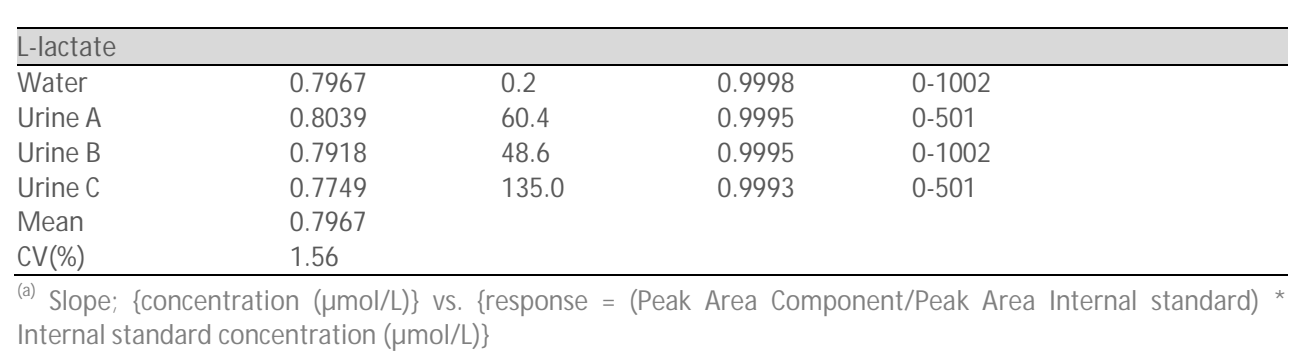


Table 4.3 Recovery data for Plasma and Urine.

\begin{tabular}{|c|c|c|c|}
\hline $\begin{array}{l}\text { PLASMA } \\
\text { D-lactate added }{ }^{(a)} \\
\mu \mathrm{mol} / \mathrm{L}(\mathrm{n}=5)\end{array}$ & Mean (SD) $\mu \mathrm{mol} / \mathrm{L}$ & $\mathrm{CV}, \%$ & Recovery, \% \\
\hline 0 & $8.1(0.2)$ & 2.9 & - \\
\hline 52.7 & $67.3(1.2)$ & 1.8 & 112.4 \\
\hline 105.4 & $127.7(3.7)$ & 2.9 & 113.5 \\
\hline \multicolumn{4}{|l|}{ L-lactate added ${ }^{(\mathrm{a})}$} \\
\hline 0 & $1365(44.6)$ & 3.3 & - \\
\hline 1504 & $2931(26.2)$ & 0.9 & 104.2 \\
\hline 3008 & $4443.2(125.1)$ & 2.8 & 102.3 \\
\hline \multicolumn{4}{|l|}{ URINE $^{(b)}$} \\
\hline D-lactate added ${ }^{(a)}$ & Mean (SD) & & \\
\hline$\mu \mathrm{mol} / \mathrm{L}(\mathrm{n}=5)$ & $\mu \mathrm{mol} / \mathrm{mmol}$ creatinine & $\mathrm{CV}, \%$ & Recovery, \% \\
\hline 0 & $0.91(0.03)$ & 3.4 & - \\
\hline 98.8 & $8.03(0.10)$ & 1.3 & 110.9 \\
\hline 197.6 & $14.34(0.29)$ & 2.0 & 104.7 \\
\hline \multicolumn{4}{|l|}{ L-lactate added ${ }^{(a)}$} \\
\hline$\overline{0}$ & $4.84(0.13$ & 2.6 & - \\
\hline 94 & $10.99(0.38)$ & 3.5 & 100.6 \\
\hline 188 & $16.56(0.14)$ & 0.8 & 96.0 \\
\hline
\end{tabular}

(a) Addition of $25 \mu \mathrm{l}$ standard solution to $25 \mu \mathrm{l}$ plasma or urine.

${ }^{\text {(b) }}$ Creatinine concentration: $15.4 \mathrm{mmol} / \mathrm{L}$.

Table 4.4 Precision data D- and L-lactate in plasma and urine.

\begin{tabular}{|c|c|c|c|c|}
\hline $\begin{array}{l}\text { Matrix } \\
\text { Intra-assay, } n=10\end{array}$ & $\begin{array}{l}\text { D-lactate } \\
\text { Mean (SD), } \mu \mathrm{mol} / \mathrm{L}\end{array}$ & $\mathrm{CV}, \%$ & $\begin{array}{l}\text { L-lactate } \\
\text { Mean (SD), } \mu \mathrm{mol} / \mathrm{L}\end{array}$ & $\mathrm{CV}, \%$ \\
\hline$\overline{\text { Plasma A }}$ & $13.0(0.7)$ & 5.1 & $1265.3(36.2)$ & 2.9 \\
\hline Plasma B & $85.7(2.5)$ & 2.9 & 6605.8 (190.4) & 2.9 \\
\hline \multicolumn{5}{|l|}{ Inter-assay, $\mathrm{n}=10$} \\
\hline Plasma A & $12.4(0.6)$ & 5.2 & $1338.7(48.8)$ & 3.6 \\
\hline Plasma B & $85.4(3.8)$ & 4.4 & $6452.6(245.8)$ & 3.8 \\
\hline Intra-assay, $\mathrm{n}=10$ & $\begin{array}{l}\text { D-lactate } \\
\text { Mean (SD), } \\
\text { umol/ mmol creatinine }\end{array}$ & & $\begin{array}{l}\text { L-lactate } \\
\text { Mean (SD), } \\
\text { umol/ mmol creatinine }\end{array}$ & $\mathrm{CV}, \%$ \\
\hline Urine $A^{(1)}$ & $0.857(0.03)$ & 3.8 & $4.40(0.27)$ & 6.0 \\
\hline${\text { Urine } \mathrm{B}^{(2)}}$ & $16.26(0.48)$ & 2.9 & $10.58(0.60)$ & 5.7 \\
\hline \multicolumn{5}{|l|}{ Inter-assay, $\mathrm{n}=10$} \\
\hline Urine $A^{(1)}$ & $0.718(0.04)$ & 5.6 & $3.75(0.33)$ & 8.8 \\
\hline Urine $\mathrm{B}^{(2)}$ & $14.90(1.05)$ & 7.0 & $8.68(0.81)$ & 9.3 \\
\hline
\end{tabular}

${ }^{(1)}$ Urine A, creatinine $15.4 \mathrm{mmol} / \mathrm{L},{ }^{(2)}$ Urine $\mathrm{B}$, creatinine $5.9 \mathrm{mmol} / \mathrm{L}$. 


\section{Comparison of the UPLC-tandem MS method vs the enzymatic assay}

We compared the proposed UPLC-tandem MS method with the enzymatic assay, by analysing plasma and D-lactate levels in rat urine with both methods. However, due to low D-lactate levels in plasma, it was not possible to analyse these samples with the enzymatic assay (data not shown). For urine, linear regression of the data resulted in the equation $y=1.08 x+50.795$, with excellent correlation $(r=0.985)$ between both techniques (figure 4.2a). However, the Bland Altman plot showed that although in the higher range both techniques are in excellent agreement, in the lower range considerately higher values were measured with the enzymatic technique as compared with the UPLC tandem MS method (figure 4.2b).
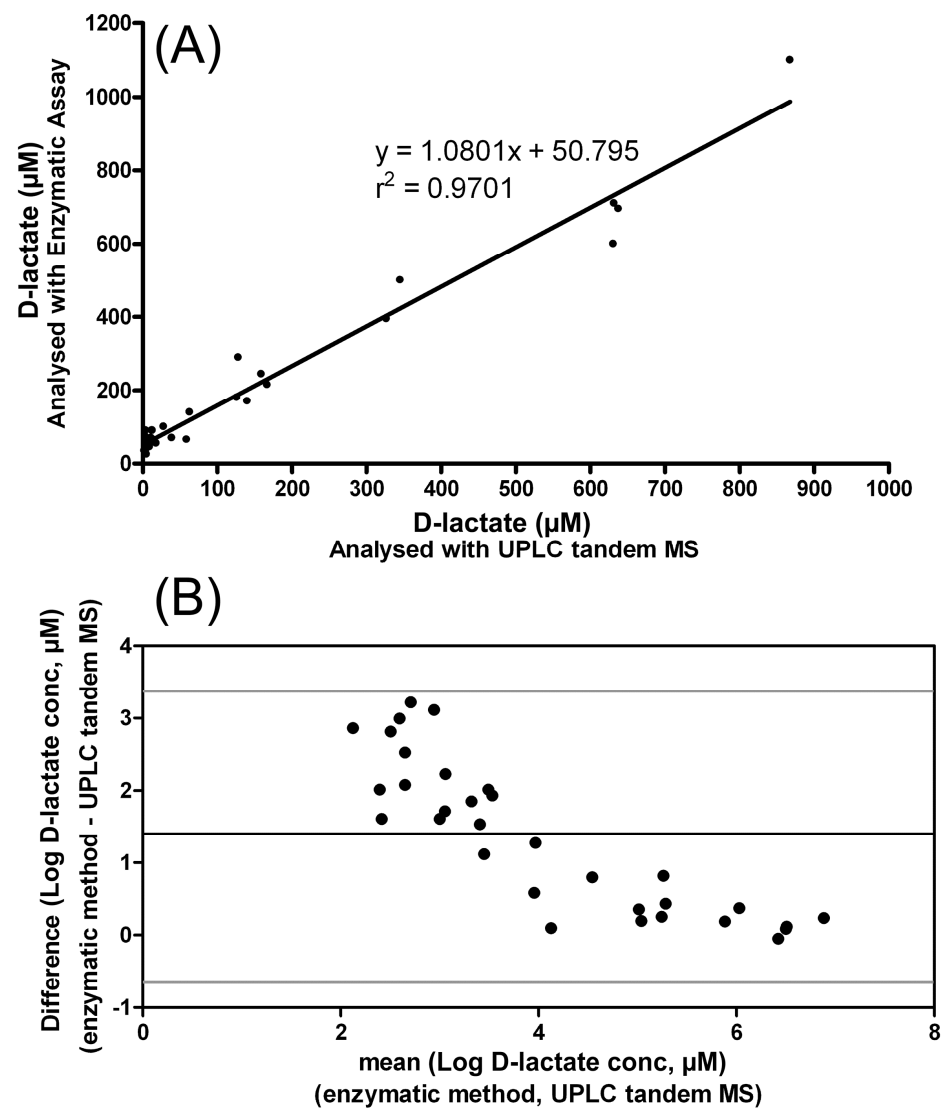

Figure 4.2. Comparison of urinary D-lactate in rat urine as measured by UPLC tandem MS and enzymatic method.

(A) Correlation between D-lactate levels measured by UPLC tandem MS and enzymatic method.

(B) Bland-Altman plot of log transformed D-lactate levels as measured by UPLC tandem MS and enzymatic method. 


\section{Comparison of urinary and plasma D- and L-lactate concentration between controls, individuals with T2DM and individuals with IBD}

We next investigated D- and L-lactate plasma and urine concentration of non-diabetic controls, patients with IBD and T2DM patients (figure 4.3). In healthy controls, D- and L-lactate concentrations were $8.0 \pm 0.6$ and $1044.8 \pm 36.7 \mu \mathrm{mol} / \mathrm{L}$ in plasma, respectively, and $1.1 \pm 0.2$ and $6.3 \pm 0.9 \mu \mathrm{mol} / \mathrm{mmol}$ creatinine in urine, respectively (mean $\pm S E M$ ). In IBD patients, levels of D- and L-lactate were higher, when compared to the non-diabetic control group in both plasma (10.7 \pm 1.2 and $1172.4 \pm 74.6 \mu \mathrm{mol} / \mathrm{L}$, respectively) and urine $(3.1 \pm 0.8$ and $11.8 \pm 1.4 \mu \mathrm{mol} / \mathrm{mmol}$ creatinine, respectively), which was significant for urinary L-lactate.

In T2DM patients, the concentrations of D- and L-lactate were significantly higher in plasma (12.3 \pm 0.8 and $1534.7 \pm 67.5 \mu \mathrm{mol} / \mathrm{L}$, respectively) and urine $(3.4 \pm 1.0$ and $12.1 \pm$ $2.0 \mu \mathrm{mol} / \mathrm{mmol}$ creatinine, respectively). Both plasma and urinary D-lactate levels, as determined in T2DM and controls, correlated with $\mathrm{HbAlC}(r=0.392, p<0.001$ and $r=0.421$, $p \varangle 0.001$, respectively) and fasting plasma glucose ( $r=0.360, p \varangle 0.001$ and $r=0.416 p \varangle 0.001$, respectively).
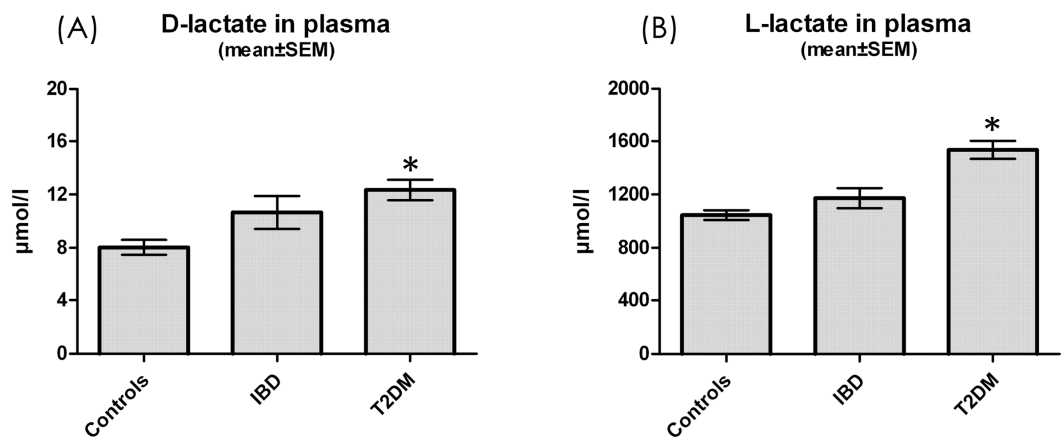

(C) D-lactate in urine

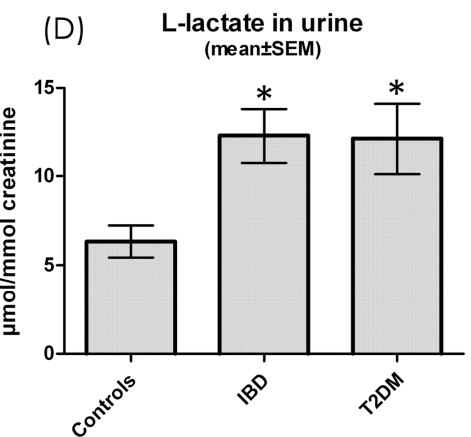

Figure 4.3. Urinary and plasma D- and L-lactate concentrations of controls, patients with inflammatory bowel disease (IBD) and patients with type 2 diabetic patients (T2DM). Data are presented as M ean $\pm \mathrm{SEM}, * \mathrm{P} \varangle 0.05$. 


\section{Discussion}

We describe here a rapid, sensitive and highly specific method for the simultaneous determination of D- and L-lactate in plasma and urine by UPLC MS/MS. The derivatisation of D- and L-lactate with DATAN makes it possible to separate both enantiomers on a reversed phase based analytical column. This results in a robust chromatographic system without the need of a column-switching or solid phase extraction (SPE) pre-sample cleanup. We found that urinary and plasma levels of D- and L-lactate were significantly increased in T2DM as compared with non-diabetic controls. In addition, we observed higher L- and D-lactate levels in patients with IBD, but only significant for urinary L-lactate.

Many other techniques have been used to quantify D- and L-lactate, with several disadvantages, such as long run-times ${ }^{3,11,26,27}$, large sample volume ${ }^{19,21,22,24,27}$, a columnswitching pre-separation technique ${ }^{3,12,13}$ or low sensitivity ${ }^{11,24,27}$. Moreover, a disadvantage of the enzymatic method is that it is not possible to measure D- and L-lactate in a single run.

Chiral stationary phase liquid chromatography has been applied for the enantiomeric separation of D- and L-lactate ${ }^{11,14,25}$. SPE or pre-reversed phase liquid chromatographic separation are obligatory for good chiral chromatographic performance, ${ }^{3,12,13,29}$. Furthermore the shorter lifetime, higher cost and difficult selection of a suitable chiral column $n^{30,31}$ has made an alternative method for enantiomeric separation desirable. More recently Cevasco et al. ${ }^{26}$ used a reversed phase liquid chromatography method for separation of D- and L-lactic acid. However; a run-to-run time of 35 minutes and an obligatory SPE sample preparation step makes this method less feasible for large cohort studies. Moreover, the used derivatisation reagent was not commercially available and had to be synthesized before use.

Anhydrides of tartaric acid were used successfully as chiral derivatisation reagents of hydroxy acids and other enantiomeric compounds ${ }^{32-34}$. In this paper we describe the derivatisation of the enantiomeric D- and L-lactate with DATAN. This derivatisation step results in a highly sensitive and specific $D$ - and L- lactate derivative which is baseline separated on a UPLC reversed phase column and detected with tandem MS. The advantage of this technique, as compared to the described methods in the literature, is that there is no need for sample clean up or pre-separation of the sample matrix and only $25 \mu \mathrm{l}$ of sample is necessary. Also the highly efficient and specific fragments of these DATAN derivatives generated in the collision cell, is an improvement against the nonderivatized analysis of $\mathrm{D}$ - and L-lactate with $\mathrm{LC} / \mathrm{MS}^{25}$. With a run-to-run time of 6 minutes we established a fast and reliable method suitable for measuring $D$ - and L-lactate in large cohort-studies.

The D- and L-lactate concentrations we measured in plasma and urine from healthy controls are in reasonable agreement with data obtained by other techniques, $3,18,19,25$. Indeed, we found an acceptable correlation of the new UPLC tandem MS method with the enzymatic assay in urine samples. 
However, the enzymatic assay is not adequately sensitive for lower levels of D-lactate, as reflected in the Bland-Altman plot. The enzymatic method measures higher levels of D-lactate than the UPLC tandem MS in the lower range.

D-lactate was not significantly increased in patients with IBD compared with non-diabetic controls in both plasma and urine. Another study, however, found a significant increase of D-lactate in hospitalised patients with active IBD 6 . This difference may be explained by the fact that the patients we have included were in remission. In addition, due to the relatively small sample size, the power to detect statistically differences was low.

We found a statistically significant increase of urine and plasma levels of D- and L-lactate in T2DM as compared with non-diabetic controls. The fact that both D-lactate and L-lactate are increased in patients with T2DM suggests that the hyperglycaemic state is an important source of D-lactate elevations in diabetes. L-lactate is mainly formed during glycolysis by conversion of pyruvate to L-lactate by lactate dehydrogenase. D-lactate is an endproduct of the metabolism of methylglyoxal, formed during hyperglycaemia, by the glyoxalase pathway ${ }^{35}$. In line with this, we demonstrate that D-lactate correlates significantly with $\mathrm{HbAlC}$, a marker for prolonged hyperglycaemia. However, based on our small study we cannot definitely conclude whether D-lactate is merely a reflection of methylglyoxal, gut-flora, or both, as several possible residual confounding factors such as BMI and gut permeability may explain the differences we observed between our patient groups.

Methylglyoxal is produced in small amount from carbohydrates, fat and protein metabolism. It is has been demonstrated that methylglyoxal is the most important precursor in the formation of advanced glycation endproducts. Methylglyoxal and methylglyoxal-derived advanced glycation endproducts are believed to be implicated in the development of diabetic vascular complications. Because D-lactate is elevated in diabetes and may be used as an indicator of methylglyoxal, the measurement of D-lactate needs to be evaluated in cohort studies with D-lactate as a possible predictor of diabetic complications. In addition, mechanistic studies are needed to elucidate the relative contribution of several metabolic pathways to the total urinary and plasma D-lactate pool, in both healthy and diabetic individuals.

In conclusion, this specific measurement of D- and L-lactate shows promise in the investigation of diabetes and metabolic diseases.

\section{Acknowledgements}

This research was performed within the framework of CTM M , the Center for Translational Molecular Medicine (www.ctmm.nl), project PREDICCt (grant 01C-104), and supported by the Netherlands Heart Foundation, Dutch Diabetes Research Foundation and Dutch Kidney Foundation. 


\section{References}

Ewaschuk, J. B., Naylor, J. M. \& Zello, G. A. D-lactate in human and ruminant metabolism. J Nutr 135, 1619-1625 (2005).

Stallknecht, B., Vissing, J. \& Galbo, H. Lactate production and clearance in exercise. Effects of training. A mini-review. Scand J M ed Sci Sports 8, 127-131 (1998).

3 Hasegawa, H. et al. Determination of serum D-lactic and L-lactic acids in normal subjects and diabetic patients by column-switching HPLC with pre-column fluorescence derivatization. Anal Bioanal Chem 377, 886-891 (2003).

Duzgun, A. P. et al. Serum D-lactate: a useful diagnostic marker for acute appendicitis. Hepatogastroenterology 54, 1483-1486 (2007).

Murray, M. J., Gonze, M. D., Nowak, L. R. \& Cobb, C. F. Serum D(-)-lactate levels as an aid to diagnosing acute intestinal ischemia. Am J Surg 167, 575-578 (1994).

Song, W. B. et al. Soluble intercellular adhesion molecule-1, D-lactate and diamine oxidase in patients with inflammatory bowel disease. World J Gastroenterol 15, 3916-3919 (2009).

Talasniemi, J. P., Pennanen, S., Savolainen, H., Niskanen, L. \& Liesivuori, J. Analytical investigation: assay of D-lactate in diabetic plasma and urine. Clin Biochem 41, 1099-1103 (2008).

Tubbs, P. K. The metabolism of D-alpha-hydroxy acids in animal tissues. Ann N Y Acad Sci 119, 920926 (1965).

Brownlee, M. Biochemistry and molecular cell biology of diabetic complications. Nature 414, 813-820 (2001).

Halperin, M. L. \& Kamel, K. S. D-lactic acidosis: turning sugar into acids in the gastrointestinal tract. Kidney Int 49, 1-8 (1996).

Ewaschuk, J. B., Naylor, J. M., Barabash, W. A. \& Zello, G. A. High-performance liquid chromatographic assay of lactic, pyruvic and acetic acids and lactic acid stereoisomers in calf feces, rumen fluid and urine. J Chromatogr B Analyt Technol Biomed Life Sci 805, 347-351 (2004).

Fukushima, T. et al. Simultaneous determination of D-lactic acid and 3-hydroxybutyric acid in rat plasma using a column-switching HPLC with fluorescent derivatization with 4-nitro-7-piperazino2,1,3-benzoxadiazole (NBD-PZ). Biomed Chromatogr 15, 189-195 (2001).

Ichihara, H., Fukushima, T. \& Imai, K. Enantiomeric determination of D- and L-lactate in rat serum using high-performance liquid chromatography with a cellulose-type chiral stationary phase and fluorescence detection. Anal Biochem 269, 379-385 (1999).

4 Okubo, S. et al. Enantiomeric determination of L- and D-lactic acid in human cerebrospinal fluid by chiral ligand exchange high-performance liquid chromatography. Biomed Chromatogr 14, 474-477 (2000).

5 Omole, O. O., Brocks, D. R., Nappert, G., Naylor, J. M. \& Zello, G. A. High-performance liquid chromatographic assay of $(H-$-)-lactic acid and its enantiomers in calf serum. J Chromatogr B Biomed Sci Appl 727, 23-29 (1999).

Demircan, M. et al. Plasma D-lactic acid level: a useful marker to distinguish perforated from acute simple appendicitis. Asian J Surg 27, 303-305 (2004).

7 Goodall, S. R. \& Byers, F. M. Automated micro method for enzymatic $L(+)$ and $D(-)$ lactic acid determinations in biological fluids containing cellular extracts. Anal Biochem 89, 80-86 (1978). 
Haschke-Becher, E., Baumgartner, M. \& Bachmann, C. Assay of D-lactate in urine of infants and children with reference values taking into account data below detection limit. Clin Chim Acta 298, 99109 (2000).

Brandt, R. B., Siegel, S. A., Waters, M. G. \& Bloch, M. H. Spectrophotometric assay for D-(-)-lactate in plasma. Anal Biochem 102, 39-46 (1980).

Ludvigsen, C. W., Thurn, J. R., Pierpont, G. L. \& Eckfeldt, J. H. Kinetic enzymic assay for D(-)-lactate, with use of a centrifugal analyzer. Clin Chem 29, 1823-1825 (1983).

Marti, R. et al. Determination of D-lactate by enzymatic methods in biological fluids: study of interferences. Clin Chem 43, 1010-1015 (1997).

Inoue, Y., Shinka, T., Ohse, M., Ikawa, H. \& Kuhara, T. Application of optical isomer analysis by diastereomer derivatization GC/MS to determine the condition of patients with short bowel syndrome. J Chromatogr B Analyt Technol Biomed Life Sci 838, 37-42 (2006).

Inoue, $Y$. et al. Changes in urinary level and configuration ratio of D-lactic acid in patients with short bowel syndrome. J Chromatogr B Analyt Technol Biomed Life Sci 855, 109-114 (2007).

Franco, E. J., Hofstetter, H. \& Hofstetter, O. Determination of lactic acid enantiomers in human urine by high-performance immunoaffinity LC-M S. J Pharm Biomed Anal 49, 1088-1091 (2009).

Norton, D. et al. High performance liquid chromatography-tandem mass spectrometry (HPLC/MS/MS) assay for chiral separation of lactic acid enantiomers in urine using a teicoplanin based stationary phase. J Chromatogr B Analyt Technol Biomed Life Sci 850, 190-198 (2007).

Cevasco, G., Piatek, A. M., Scapolla, C. \& Thea, S. A simple, sensitive and efficient assay for the determination of $D$ - and L-lactic acid enantiomers in human plasma by high-performance liquid chromatography. J Chromatogr A 1218, 787-792 (2011).

Tan, L., Wang, Y., Liu, X., Ju, H. \& Li, J. Simultaneous determination of I- and d-lactic acid in plasma by capillary electrophoresis. J Chromatogr B Analyt Technol Biomed Life Sci 814, 393-398 (2005).

Jaffé, M. Ueber die Niederschlag, welchen Pikrinsaure in

normalem Ham erzeugt und uber eine neue Reaction des

Kreatinins. Hoppe-Seylers. Z. physiol. Chem 10, 391 (1886).

Buglass, A. J. \& Lee, S. H. Sequential analysis of malic acid and both enantiomers of lactic acid in wine using a high-performance liquid chromatographic column-switching procedure. J Chromatogr Sci 39, 453-458 (2001).

Krstulovic, A. M. Chiral stationary phases for the liquid chromatographic separation of pharmaceuticals. J Pharm Biomed Anal 6, 641-656 (1988).

Persson, B. A. \& Andersson, S. Unusual effects of separation conditions on chiral separations. J Chromatogr A 906, 195-203 (2001).

Lindner, W. \& Hirschbock, I. Tartaric acid derivatives as chiral sources for enantioseparation in liquid chromatography. J Pharm Biomed Anal 2, 183-189 (1984).

Struys, E. A., Jansen, E. E., Verhoeven, N. M. \& Jakobs, C. Measurement of urinary D- and L-2hydroxyglutarate enantiomers by stable-isotope-dilution liquid chromatography-tandem mass spectrometry after derivatization with diacetyl-L-tartaric anhydride. Clin Chem 50, 1391-1395 (2004).

Sun, X. X., Sun, L. Z. \& Aboul-Enein, H. Y. Chiral derivatization reagents for drug enantioseparation by high-performance liquid chromatography based upon pre-column derivatization and formation of diastereomers: enantioselectivity and related structure. Biomed Chromatogr 15, 116-132 (2001). Thornalley, P. J. The glyoxalase system in health and disease. M ol Aspects M ed 14, 287-371 (1993). 



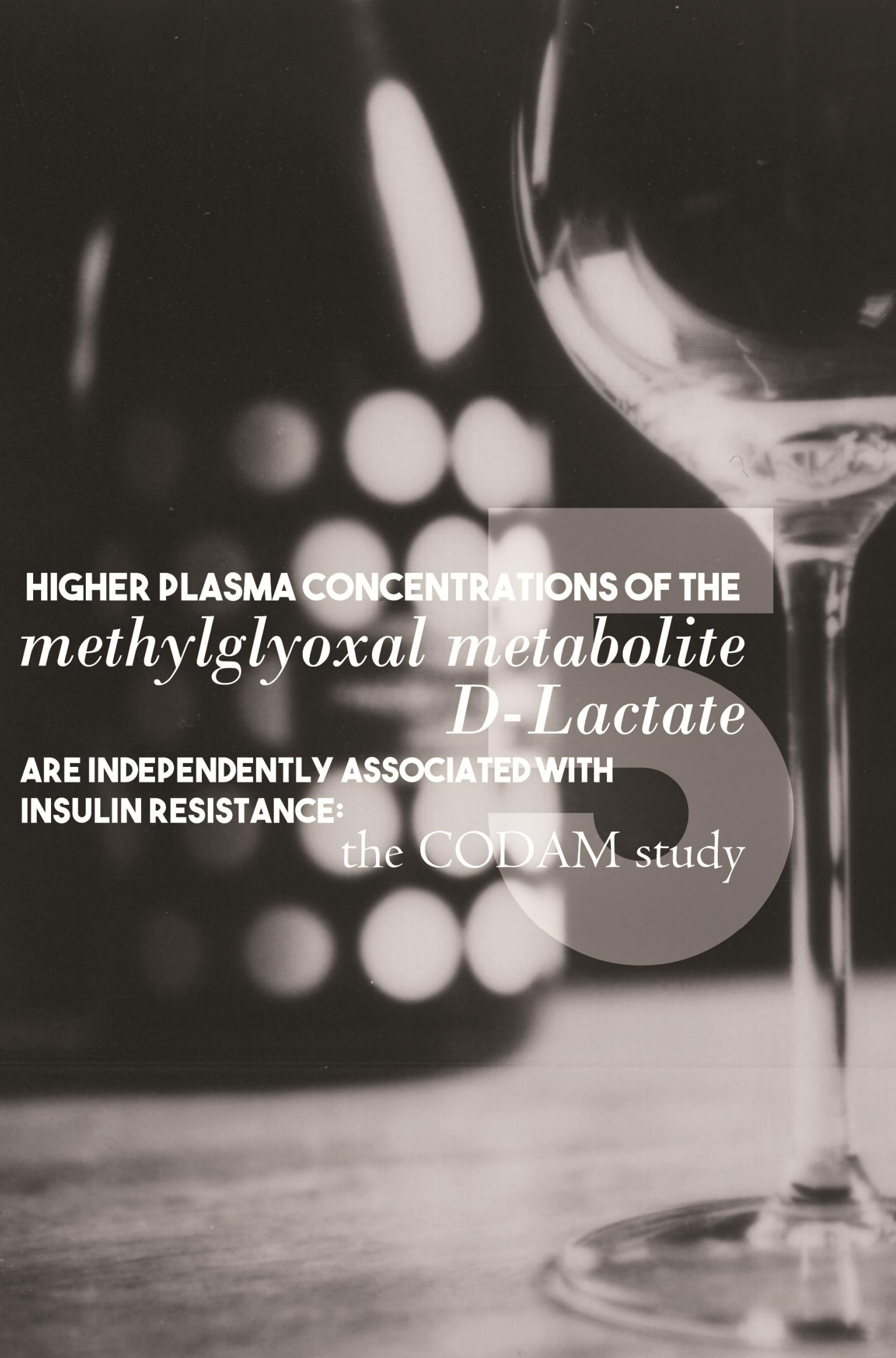




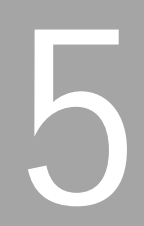

Higher plasma concentrations of the methylglyoxal metabolite D-lactate are independently associated with insulin resistance: the CODAM study

Dionne E.M. Maessen, Jean L.J.M. Scheijen, Katrien H. Gaens, Marleen M .J. van Greevenbroek, Carla J. van der Kallen, Coen D.A. Stehouwer, Casper G. Schalkwijk Journal of Diabetes \& M etabolism 5:11; (2014) 


\section{Abstract}

\section{Objective:}

Methylglyoxal (MGO), a highly reactive dicarbonyl compound generated by the spontaneous degradation of glycolytic intermediates, is a major precursor for advanced glycation endproducts and can potentially disrupt cellular functions. MGO can be detoxified by the glyoxalase system into D-lactate. Although experimental studies have shown that increased levels of MGO are associated with insulin resistance, epidemiological evidence of such an association in human studies is lacking. The aim of this study was to investigate the association between plasma D-lactate, as a reflection of plasma MGO concentrations, and insulin resistance.

\section{Methods:}

Cross-sectional, complete case analyses were performed in the Cohort study on Diabetes and Atherosclerosis Maastricht (CODAM). 513 participants were included in the study population: $59.4 \pm 6.9$ years of age, $63 \%$ men, and, by design, $23 \%$ impaired glucose metabolism (IGM) and 23\% type 2 diabetes mellitus (T2DM). Plasma D-lactate was measured by UPLC-M S/MS. The main outcome measure was HOM A2-IR, as a measure of insulin resistance. The association between plasma D-lactate and HOM A2-IR was studied by multiple linear regression analysis.

\section{Results:}

The prevalence of T2DM increased significantly over the tertiles of plasma D-lactate concentrations. Moreover, plasma D-lactate was positively associated with HOMA2-IR, when adjusted for age and sex ( $\beta=0.429 ; 95 \% \mathrm{Cl}: 0.350-0.507 ; \beta \varangle 0.001)$, as well as in the fully adjusted model, additionally adjusted for glucose metabolism status, smoking status, cardiovascular disease, use of medication (glucose-, lipid-, and blood-pressure-lowering), estimated glomerular filtration rate, waist circumference, glycated hemoglobin (HbA1C) and plasma L-lactate levels ( $\beta=0.145 ; 95 \% \mathrm{Cl}$ : 0.051-0.239; $p=0.003$ ). Additional data indicate that other sources of $\mathrm{D}$-lactate can be excluded.

\section{Conclusion:}

We found a positive association between plasma D-lactate and HOM A2-IR, independently of putative confounders. These results suggest that MGO plays a role in insulin resistance, although direct measurement of MGO is necessary to confirm this. 


\section{Introduction}

Insulin resistance with respect to glucose metabolism, is a metabolic condition defined by a reduced responsiveness to the action of insulin on glucose uptake, metabolism or storage ${ }^{1}$. Recently, increased levels of advanced glycation endproducts (AGEs) have been found in association with insulin resistance ${ }^{1-3}$.

AGEs are a family of unavoidable by-products of various metabolic pathways. Methylglyoxal (MGO) has received considerable attention as the most reactive and potent AGE precursor $r^{4,5}$. MGO is a dicarbonyl compound generated by the spontaneous degradation of glycolytic intermediates during glycolysis. Under physiological circumstances, MGO can be detoxified by the glyoxalase (GLO) system. This system consists of two enzymes, glyoxalase 1 (GLO-1) and glyoxalase 2 (GLO-2), which catalyze the conversion of M GO to D-lactate ${ }^{6}$.

Although several in vitro experiments and animal studies have indicated a substantial role for MGO in the induction of insulin resistance ${ }^{1,7}$, the association between MGO and insulin resistance in humans has not yet been studied. However, because of the high reactivity of MGO with plasma proteins, it is difficult to obtain valid and precise measurements of MGO in plasma. Since MGO is metabolized to D-lactate, we used plasma D-lactate levels as a reflection of M GO concentrations in the current analysis ${ }^{8}$. We previously demonstrated that we are able to measure D-lactate in a very specific way that can efficiently distinguish between D-lactate and L-lactate ${ }^{9}$. MGO degradation is the major route of D-lactate formation, although D-lactate can also originate from other sources such as the intestines. In previous work, however, we found that plasma levels of D-lactate were significantly increased in patients with type 2 diabetes mellitus (T2DM) compared to healthy controls, whereas patients suffering from inflammatory bowel disease (IBD) did not show increased levels of plasma D-lactate. These data underline that increased concentrations of plasma D-lactate are derived from MGO and are not a reflection of D-lactate produced by the intestines.

In this study, we investigated if plasma D-lactate, as a reflection of M GO concentrations, is increased in individuals with impaired glucose metabolism (IGM) and T2DM, and whether plasma D-lactate is associated with insulin resistance in a cohort study designed to investigate glucose metabolism and cardiovascular disease. 


\section{Materials and methods}

\section{Study population}

The study population consisted of participants from the Cohort study on Diabetes and Atherosclerosis Maastricht (CODAM). This study included 574 participants who were selected from the general population, as described in detail elsewhere ${ }^{10}$.

The cohort was enriched for impaired glucose metabolism status, as defined previously ${ }^{11}$. Current analyses were performed on participants who were not on insulin treatment and had complete data on all variables of interest (plasma D-lactate, HOMA2-IR, age, sex, glucose metabolism status, smoking status, cardiovascular disease, use of medication (glucose-, lipid-, and blood-pressure-lowering), estimated glomerular filtration rate (eGFR), waist circumference, glycated hemoglobin ( $\mathrm{HbA1C}$ ) and plasma L-lactate levels). Hence, the present study reports on 513 persons. The study protocol was approved by the local M edical Ethical Committee of M aastricht University M edical Centre and written informed consent was obtained from all participants.

\section{M easurement of plasma D-lactate}

Plasma D-lactate levels were measured in EDTA plasma samples from overnight fasting venous blood. Samples were analyzed by reversed phase ultra-performance liquid chromatography tandem mass spectrometry (UPLC-MS/MS), as described in detail previously ${ }^{9}$. The intra- and inter-assay variations were 4 and $8 \%$, respectively.

\section{Measurement of insulin resistance}

Insulin resistance was estimated using the Homeostasis Model of Assessment - Insulin Resistance (HOM A2-IR) calculator from www.dtu.ox.ac.uk, as described previously ${ }^{12}$.

\section{M easurements of covariates}

Plasma L-lactate levels were measured in EDTA plasma samples from overnight fasting venous blood, using UPLC-MS/MS ${ }^{9}$. It was measured synchronously with plasma D-lactate. Smoking behavior (never, ever or current smoker) was assessed by a questionnaire. Prior CVD and waist circumference were assessed as previously described in detail ${ }^{13}$.

Creatinine levels were measured in EDTA plasma using a Jaffe diagnostic test (Roche Diagnostics) and the Chronic Kidney Disease Epidemiology Collaboration (CKD-EPI) equation was used to calculate the $\mathrm{GFR}^{14}$. Finally, HbAlC was measured using ionexchange HPLC ${ }^{10}$. 


\section{Use of medication}

Subjects were asked to report if they were currently using medication, and, if so, to list all the medication used. All medicines were reviewed with the use of the Dutch Pharmacological Compass 2006 (www.fk.cvz.nl) and allocated into 36 categories. Medication containing the additive polyethylene glycol (PEG) was selected using the database of College ter Beoordeling van Geneesmiddelen (www.dbg-med.nl).

\section{Definition of intestinal disease}

Diseases of study participants were self-reported by questionnaire. Intestinal disease was reported when the subject suffered from any serious intestinal disease for 3 months or longer in the past 12 months.

\section{Statistical analyses}

The general characteristics of the study population were compared across tertiles of plasma D-lactate concentrations. One-way analysis of variance (ANOVA) was used for continuous variables and chi-square tests for discrete variables. Skewed variables (plasma D-lactate, HOM A2-IR, fasting plasma glucose, plasma L-lactate and triglycerides) were compared across the plasma D-lactate tertiles by Kruskal-Wallis one-way analysis of variance and were $\log ^{e}$ transformed prior to regression analyses. Multiple linear regression analysis was used to investigate the cross-sectional association between plasma D-lactate and HOMA2-IR and results are presented as standardized regression coefficients $(\beta)$. The analysis was initially adjusted for the covariates age (years) and sex (model 1), additionally adjusted for glucose metabolism status (IGM and T2DM as dummy variables), smoking status (ex-smoker and current smoker as dummy variables), prior cardiovascular disease, use of medication (glucose-, lipid-, and blood-pressure-lowering) and eGFR (model 2), further adjusted for HbAlC (model 3), and finally fully adjusted for plasma L-lactate levels (model 4). A P-value of $<0.05$ was considered statistically significant. All statistical analyses were performed with IBM SPSS Statistics Software, version 20 (IBM Corporation, Armonk, New York). 


\section{Results}

General characteristics of the study population stratified according to tertiles of plasma D-lactate concentrations are shown in table 5.1. The prevalence of IGM did not differ between the tertiles of plasma D-lactate levels (table 5.1). However, the prevalence of T2DM increased significantly with higher plasma D-lactate levels $(p \varangle 0.001)$ (table 5.1). Similar results were found for fasting plasma glucose levels, HOMA2-IR, and HbA1C (all $p \varangle 0.001$ ) (table 5.1).

Table 5.1. General characteristics of the study population stratified according to tertiles of plasma D-lactate.

\begin{tabular}{|c|c|c|c|c|}
\hline & \multicolumn{3}{|c|}{ Plasma D-lactate tertiles } & \multirow[b]{2}{*}{ P-value } \\
\hline & Lowest $(n=171)$ & Middle $(n=171)$ & Highest $(n=171)$ & \\
\hline Age, years & $59.2 \pm 6.9$ & $59.9 \pm 7.1$ & $59.1 \pm 6.8$ & 0.43 \\
\hline Sex, \% men & 57.3 & 66.1 & 64.9 & 0.19 \\
\hline Body mass index, $\mathrm{kg} / \mathrm{m}^{2}$ & $27.3 \pm 3.7$ & $28.0 \pm 4.1$ & $30.0 \pm 4.4$ & $\varangle 0.001$ \\
\hline Waist circumference, cm & $95.5 \pm 10.9$ & $98.6 \pm 11.2$ & $103.5 \pm 12.0$ & $\varangle 0.001$ \\
\hline IGM, \% & 23.4 & 20.5 & 25.1 & 0.58 \\
\hline T2DM , \% & 11.7 & 21.6 & 35.1 & $\varangle 0.001$ \\
\hline Fasting plasma glucose, $\mathrm{mmol} / \mathrm{L}$ & $5.4[5.0-5.8]$ & $5.6[5.2-6.1]$ & $5.9[5.4-7.1]$ & $\varangle 0.001$ \\
\hline HOM A2-IR & $0.9[0.7-1.3]$ & $1.2[0.9-1.6]$ & $1.4[1.0-2.3]$ & $\varangle 0.001$ \\
\hline Plasma D-lactate, $\mu \mathrm{mol} / \mathrm{L}$ & $5.5[4.6-6.3]$ & $8.5[7.8-9.7]$ & $14.5[12.6-17.1]$ & $\varangle 0.001$ \\
\hline Plasma L-lactate, $\mu \mathrm{mol} / \mathrm{L}$ & 922 [795-1054] & 1143[974-1315] & 1634 [1358-1884] & $\varangle .001$ \\
\hline $\mathrm{HbA}_{1 \mathrm{c}}, \%$ & $5.7 \pm 0.7$ & $5.9 \pm 0.7$ & $6.1 \pm 0.8$ & $\varangle 0.001$ \\
\hline Glucose-lowering medication, \% & 5.3 & 9.4 & 17.0 & 0.002 \\
\hline Prior CVD, \% & 21.6 & 26.9 & 32.7 & 0.07 \\
\hline Total cholesterol, mmol/L & $4.2 \pm 1.3$ & $4.8 \pm 1.6$ & $5.2 \pm 1.7$ & $\varangle 0.001$ \\
\hline Triglycerides, $\mathrm{mmol} / \mathrm{L}$ & $1.1[0.8-1.5]$ & $1.4[1.0-1.9]$ & $1.8[1.4-2.4]$ & $\varangle 0.001$ \\
\hline Lipid-lowering medication, \% & 15.8 & 17.0 & 20.5 & 0.50 \\
\hline Current smoker, \% & 20.5 & 19.3 & 22.8 & 0.72 \\
\hline Former smoker, \% & 52.0 & 51.5 & 51.5 & 0.99 \\
\hline BP-lowering medication, \% & 28.7 & 38.0 & 46.2 & 0.004 \\
\hline $\mathrm{eGFR}, \mathrm{mL} / \mathrm{min} / 1.73 \mathrm{~m}^{2}$ & $90.5 \pm 11.5$ & $90.2 \pm 12.6$ & $93.8 \pm 12.1$ & 0.009 \\
\hline
\end{tabular}

Data are presented as means \pm SD, medians (interquartile range), or percentages, as appropriate; the ranges of the plasma D-lactate tertiles were [2.6-7.1], [7.1-10.7] and [10.7-32.4] $\mu \mathrm{mol} / \mathrm{L}$ respectively. IGM: impaired glucose metabolism; T2DM: type 2 diabetes mellitus; HOMA2-IR: homeostasis model assessment insulin resistance; HbAlC: glycated hemoglobin; CVD: cardiovascular disease; BP: blood-pressure; eGFR: estimated glomerular filtration rate.

Plasma D-lactate concentrations were positively associated with HOM A2-IR, in the model adjusted for age and sex (Model $1: \beta=0.429 ; 95 \% \mathrm{Cl}: 0.350-0.507 ; p \varangle 0.001$ ), as well as in a model adjusted for the additional covariates glucose metabolism status, smoking status, cardiovascular disease, use of medication, eGFR and waist circumference (Model 2: $\beta=0.214 ; 95 \% \mathrm{Cl}: 0.147-0.280 ; p \varangle 0.001)$. 
After additional adjustment for $\mathrm{HbA1C}$, which had a negligible effect on the regression coefficient, plasma D-lactate remained positively associated with HOMA2-IR (Model 3: $\beta=0.210 ; 95 \% \mathrm{Cl}: 0.143-0.276 ; p<0.001$ ).

Addition of plasma L-lactate levels to model 3 resulted in a relatively large decrease in the regression coefficient (Model $4: \beta=0.145 ; 95 \% \mathrm{Cl}: 0.051-0.239 ; p=0.003$ ), although the association between plasma D-lactate and HOM A2-IR remained significant (Table 5.2).

Table 5.2. Association between plasma D-lactate ( $\mu \mathrm{mol} / \mathrm{L})$ and HOM A2-IR.

\begin{tabular}{lllll}
\hline Dependent variable & Model & $\beta$ & $95 \% \mathrm{Cl}$ & P-value \\
\hline \multirow{3}{*}{ HOM A2-IR } & 1 & 0.429 & $0.350-0.507$ & $\varangle .001$ \\
& 2 & 0.214 & $0.147-0.280$ & $\varangle 0.001$ \\
& 3 & 0.210 & $0.143-0.276$ & $\varangle 0.001$ \\
\hline
\end{tabular}

The standardized regression coefficient $\beta$ represents one standard deviation (SD) increase in HOM A2-IR per SD increase in plasma D-lactate.

Model 1: adjusted for age and sex;

Model 2: model 1 + glucose metabolism status, smoking status, cardiovascular disease, use of medication (glucose-, lipid-, and blood-pressure-lowering), estimated glomerular filtration rate and waist circumference; Model 3: model $2+\mathrm{HbAlC}$;

Model 4: model 3 + plasma L-lactate levels.

Although M GO degradation is the major route of D-lactate formation, D-lactate can also be produced by other pathways. We already found in our previous study that patients suffering from IBD did not show increased levels of plasma D-lactate. However, we additionally analyzed our study population with regard to intestinal disease. There were no differences in prevalence of intestinal disease between the plasma D-lactate tertiles (T1: $1.8 \%, T 2: 4.7 \%, T 3: 2.9 \% ; p=0.29$ ), nor was there a difference in the proportion of intestinal diseased participants treated for this disease (T1: 50\%, T2: 57\%, T3: 44\%; $p=0.88$ ).

In addition to the intestinal route, D-lactate can also be formed from PEG, which is used as an additive for several medications. To exclude PEG as a source of D-lactate production, we selected all medication in our cohort which was enriched with PEG, and analyzed whether there were more users of PEG-contaminated medication in the higher tertile of plasma D-lactate. We found that the users of PEG-contaminated medication were equally distributed between the tertiles of D-lactate (T1: $35 \%, T 2: 40 \%$, T3: $46 \%$; $p=0.14)$. Since PEG is often added to BP-lowering medication, we performed the same analyses for this specific group of medication. Again, no difference in amount of users was found between the D-lactate tertiles ( $T 1: 57 \%, T 2: 66 \%, T 3: 60 \% ; p=0.58$ ). 


\section{Discussion}

In this cohort study we demonstrated that plasma D-lactate levels are increased in patients with T2DM. Additionally, we found a positive association between plasma D-lactate levels and HOM A2-IR, independently of putative confounders.

This is the first study that has examined the association between MGO and insulin resistance in a large human cohort study, using plasma D-lactate as a reflection of MGO concentrations. We observed a significantly higher prevalence of T2DM across the tertiles of plasma D-lactate concentrations. A similar trend was observed for fasting plasma glucose levels, HOMA2-IR, and $\mathrm{HbAlC}$, supporting the hypothesis that persons with abnormal glucose metabolism have high plasma D-lactate levels. These positive associations are in line with our previous study, in which we demonstrated that T2DM patients have increased plasma and urine levels of D-lactate ${ }^{9}$ and with studies showing that the concentration of D-lactate typically increased in cells cultured in high glucose concentrations ${ }^{5,8}$.

We found a strong association between plasma D-lactate and HOMA2-IR, which was independent of confounders. Although adjustment for plasma L-lactate levels resulted in a relatively large decrease in the regression coefficient, the association between plasma D-lactate and HOMA2-IR remained significant, demonstrating an association between plasma D-lactate probably derived from MGO metabolism and insulin resistance. Our results are in agreement with several experimental studies showing that the major AGEprecursor MGO potentially contributes to the development of insulin resistance. It has been demonstrated that administration of MGO to rats caused impaired insulin signaling and induced insulin resistance ${ }^{15-18}$. In addition, chronic infusion of MGO caused $\beta$-cell dysfunction ${ }^{18}$. Next to the in vivo studies, in vitro experiments with 3T3 adipocytes and INS-1E pancreatic cells showed that IRS-1 phosphorylation and PI3K activity were dosedependently reduced by treatment with MGO, indicating its contribution to the pathogenesis of insulin resistance ${ }^{17,19}$.

Our finding that plasma D-lactate is positively and independently associated with insulin resistance is in line with findings from recent studies, which described a positive association between AGEs and insulin resistance ${ }^{1-3}$. However, we cannot establish whether these associations are causal due to the cross-sectional design of the study. Further follow-up data on these or other patient groups would, of course, be invaluable, in testing whether our findings are probably due to causal relationships between MGO and insulin resistance. However, this cross-sectional study may serve as a reasonable starting point to further explore these associations. Plasma D-lactate may be, as a reflection of plasma M GO, an early marker of insulin resistance.

The main limitation of our study is that we were not able so far to measure M GO directly in these plasma samples. However, it is very likely that an increase in D-lactate concentrations is indicative of increased flux of M GO formation, as was demonstrated in erythrocytes ${ }^{8}$. Furthermore, our additional analyses showed that we can exclude other sources than MGO for the production of D-lactate. 
Previous work and current results indicate that both the intestines as well as PEGcontaminated medication do not contribute to plasma D-lactate levels.

In summary, our data show for the first time that plasma D-lactate levels are associated with HOMA2-IR, independently of putative confounders. Together with findings from previous studies, our results suggest that higher levels of M GO play an important role in the etiology of insulin resistance, although this has to be confirmed in future research. 


\section{References}

1 Schalkwijk, C. G., Brouwers, O. \& Stehouwer, C. D. Modulation of insulin action by advanced glycation endproducts: a new player in the field. Hormone and metabolic research $=$ Hormon- und Stoffwechselforschung $=$ Hormones et metabolisme 40, 614-619, doi:10.1055/s-0028-1082085 (2008). Sandu, 0 . et al. Insulin resistance and type 2 diabetes in high-fat-fed mice are linked to high glycotoxin intake. Diabetes 54, 2314-2319 (2005).

3 Forbes, J. M. et al. Advanced glycation end products (AGEs) are cross-sectionally associated with insulin secretion in healthy subjects. Amino acids 46, 321-326, doi:10.1007/s00726-013-1542-9 (2014).

4 Westwood, M. E. \& Thornalley, P. J. M olecular characteristics of methylglyoxal-modified bovine and human serum albumins. Comparison with glucose-derived advanced glycation endproduct-modified serum albumins. Journal of protein chemistry 14, 359-372 (1995).

Shinohara, M. et al. Overexpression of glyoxalase-I in bovine endothelial cells inhibits intracellular advanced glycation endproduct formation and prevents hyperglycemia-induced increases in macromolecular endocytosis. The Journal of clinical investigation 101, 1142-1147, doi:10.1172/JCl119885 (1998).

6 Thornalley, P. J. The glyoxalase system in health and disease. Molecular aspects of medicine 14, 287371 (1993).

7 Matafome, P., Sena, C. \& Seica, R. Methylglyoxal, obesity, and diabetes. Endocrine 43, 472-484, doi:10.1007/s12020-012-9795-8 (2013).

8 Thornalley, P. J. Modification of the glyoxalase system in human red blood cells by glucose in vitro. The Biochemical journal 254, 751-755 (1988).

9 Scheijen, J. L. et al. $L(+)$ and $D(-)$ lactate are increased in plasma and urine samples of type 2 diabetes as measured by a simultaneous quantification of $L(+)$ and $D(-)$ lactate by reversed-phase liquid chromatography tandem mass spectrometry. Experimental diabetes research 2012, 234812, doi:10.1155/2012/234812 (2012).

$10 \mathrm{Du}, \mathrm{H}$. et al. Glycemic index and glycemic load in relation to food and nutrient intake and metabolic risk factors in a Dutch population. The American journal of clinical nutrition 87, 655-661 (2008).

11 Hanssen, N. M. et al. Plasma levels of advanced glycation endproducts Nepsilon(carboxymethyl)lysine, Nepsilon-(carboxyethyl)|ysine, and pentosidine are not independently associated with cardiovascular disease in individuals with or without type 2 diabetes: the Hoorn and CODAM studies. The Journal of clinical endocrinology and metabolism 98, E1369-1373, doi:10.1210/jc.2013-1068 (2013).

12 Wlazlo, N. et al. Iron metabolism is associated with adipocyte insulin resistance and plasma adiponectin: the Cohort on Diabetes and Atherosclerosis Maastricht (CODAM) study. Diabetes care 36, 309-315, doi:10.2337/dc12-0505 (2013).

13 van Greevenbroek, M. M. et al. The cross-sectional association between insulin resistance and circulating complement $\mathrm{C} 3$ is partly explained by plasma alanine aminotransferase, independent of central obesity and general inflammation (the CODAM study). European journal of clinical investigation 41, 372-379, doi:10.1111/j.1365-2362.2010.02418.x (2011). 
14 Levey, A. S. et al. A new equation to estimate glomerular filtration rate. Annals of internal medicine 150, 604-612 (2009).

15 Guo, Q. et al. Methylglyoxal contributes to the development of insulin resistance and salt sensitivity in Sprague-Dawley rats. Journal of hypertension 27, 1664-1671, doi:10.1097/HJH.0b013e32832c419a (2009).

16 Dhar, A., Desai, K. M. \& Wu, L. Alagebrium attenuates acute methylglyoxal-induced glucose intolerance in Sprague-Dawley rats. British journal of pharmacology 159, 166-175, doi:10.1111/j.1476-5381.2009.00469.x (2010).

17 Jia, X.\& Wu, L. Accumulation of endogenous methylglyoxal impaired insulin signaling in adipose tissue of fructose-fed rats. Molecular and cellular biochemistry 306, 133-139, doi:10.1007/s11010007-9563-x (2007).

18 Dhar, A., Dhar, I., Jiang, B., Desai, K. M. \& Wu, L. Chronic methylglyoxal infusion by minipump causes pancreatic beta-cell dysfunction and induces type 2 diabetes in Sprague-Dawley rats. Diabetes $\mathbf{6 0}$, 899-908, doi:10.2337/db10-0627 (2011).

19 Fiory, F. et al. Methylglyoxal impairs insulin signalling and insulin action on glucose-induced insulin secretion in the pancreatic beta cell line INS-1E. Diabetologia 54, 2941-2952, doi:10.1007/s00125011-2280-8 (2011). 
QUANIIFICATION OF glyoxal, methylglyoxal and 3 deoxyghucosone NBLOOD AND PLASMA BY ULTRA DER=ORMANCE HOUIDCHROMAIOCRAPHY

tandem mass spectrometry; EVALUATION OF BLOOD SPECIMEN 
Quantification of glyoxal, methylglyoxal and 3-deoxyglucosone in blood and plasma by ultra-performance liquid chromatography tandem mass spectrometry; evaluation of blood specimen

Jean LJ.M. Scheijen and Casper G. Schalkwijk

Clin Chem Lab M ed 52(1); 85-91 (2014) 


\section{Abstract}

Background: The reactive $\alpha$-oxoaldehydes glyoxal (GO), methylglyoxal (MGO) and 3-deoxyglucosone (3-DG) have been linked to diabetic complications and other agerelated diseases. Numerous techniques have been described for the quantification of $\alpha$-oxoaldehydes in blood or plasma, albeit with several shortcomings such as the need of large sample volume, elaborate extraction steps or long run-times during analysis. Therefore, we developed and evaluated an improved method including sample preparation, for the quantification of these $\alpha$-oxoaldehydes in blood and plasma with ultra-performance liquid chromatography tandem mass spectrometry (UPLCM S/M S).

Methods: EDTA plasma and whole blood samples were deproteinized using perchloric acid (PCA) and subsequently derivatized with o-pheneylenediamine (OPD). GO, MGO and 3-DG concentrations were determined using stable isotope dilution UPLC MS/MS with a run-to-run time of 8 minutes. Stability of $\alpha$-oxoaldehyde concentrations in plasma and whole blood during storage was tested. The concentration of GO, MGO and 3-DG was measured in EDTA plasma of non-diabetic controls and patients with type 2 diabetes (T2DM).

Results and conclusion: Calibration curves of GO, M GO and 3-DG were linear throughout selected ranges. Recoveries of these $\alpha$-oxoaldehydes were between 95 and $104 \%$. Intraand inter-assay CVs were between 2 and $14 \%$.

To obtain stable and reliable $\alpha$-oxoaldehyde concentrations, immediate centrifugation of blood after blood sampling is essential and the use of EDTA as anticoagulant is preferable. Moreover, immediate precipitation of plasma protein with PCA stabilized $\alpha$-oxoaldehyde concentrations for at least 120 minutes. With the use of the developed method, we found increased plasma concentrations of GO, M GO and 3-DG in T2DM as compared to nondiabetic controls. 


\section{Introduction}

Prolonged exposure to hyperglycemia has detrimental effects on various cellular functions and is believed to be the most important factor in the development of vascular complications in diabetes. One of the hypotheses about how hyperglycemia leads to complications is the formation of advanced glycation endproducts (AGEs). In addition to the formation of AGEs by the classical Maillard reaction, $\alpha$-oxoaldehydes such as methylglyoxal (MGO), glyoxal (GO) and 3-deoxyglucocone (3-DG) are also known to form AGES $^{1-3}$. These $\alpha$-oxoaldehydes and AGEs have been linked to diabetic complications as well as other age-related human diseases ${ }^{4-10}$. To further investigate the putative role of these $\alpha$-oxoaldehydes in several diseases a specific, rapid and sensitive method is needed.

So far, $\alpha$-oxoaldehydes have been analyzed by several techniques including reversed phase liquid chromatography with fluorescence or UV detection ${ }^{2,11-15}$ or liquid chromatography with mass spectrometry detection ${ }^{1,8,16-18}$. However, these techniques have several shortcomings such as large sample volume $e^{1,8,11,13}$, elaborate liquid-liquidextraction (LLE) or solid phase extraction (SPE) steps $\mathrm{s}^{1,2,8,11,16,17}$ and long run-times ${ }^{2,12,15}$.

In the present study we developed a highly sensitive, specific and rapid ultra-performance liquid chromatography (UPLC) tandem mass spectrometry (MS/MS) method for the analysis of GO, M GO and 3-DG. We achieved a significant improvement over the methods described in literature with the use of a stable isotope labeled internal standard. Since variations in the blood draw and pretreatment of blood or plasma can also substantially influence the free concentrations of these $\alpha$-oxoaldehydes, we evaluated blood specimen and the effect of immediate precipitation of proteins in plasma or whole blood with perchloric acid (PCA) on the concentration of $\alpha$-oxoaldehydes. With an optimized method, we quantified the concentration of GO, MGO and 3-DG in EDTA plasma samples of non-diabetic controls and patients with type 2 diabetes (T2DM ).

\section{Materials and methods}

\section{Chemicals}

Glyoxal (GO) solution ( $40 \%)$, methylglyoxal (MGO) solution ( $\sim 40 \%)$, formic acid (p.a. for mass spectroscopy) and perchloric acid (PCA, 70\%, p.a.) were obtained from SigmaAldrich (Steinheim, Germany). D8-0-phenylenediamine (OPD) (98.6\%) was obtained from CDN-isotopes (Quebec, Canada). 3-Deoxyglucosone (3-DG) (95\%) was obtained from Santa Cruz (Santa Cruz, California). 0-phenylenediamine was obtained from Merck (Darmstadt, Germany). Water and acetonitrile (ULC/MS quality) were obtained from Biosolve Chimie (Dieuze, France). EDTA and EDTA + sodium fluoride (NaF) tubes were obtained by Greiner Bio-One (Vacuette Premium Tubes, Kremsmünster, Austria). 


\section{Preparation internal standard oxoaldehydes}

Internal standards for GO, M GO and 3-DG were not commercially available. Therefore, a derivatized internal standard was prepared by adding $140 \mu \mathrm{l} \mathrm{GO}(17.5 \mathrm{mmol} / \mathrm{L}), 40 \mu \mathrm{L}$ MGO $(6.5 \mathrm{mmol} / \mathrm{L})$ and $50 \mu \mathrm{L}$ 3-DG $(5.3 \mathrm{mmol} / \mathrm{L})$ to $50 \mathrm{ml} 265 \mathrm{mmol} / \mathrm{L}$ formic acid. $\mathrm{D}_{8}-0 \mathrm{PD}$ was added to a final concentration of $4.3 \mathrm{mmol} / \mathrm{L}$. This mixture was allowed to stand for 1 week at $4^{\circ} \mathrm{C}$ shielded from light. Aliquots of this solution were stored at $-80^{\circ} \mathrm{C}$ until usage.

\section{Evaluation of blood specimen}

To examine the concentration of GO, M GO and 3-DG in blood and plasma samples during handling and storage, blood specimens of 2 healthy controls were collected in EDTA and EDTA + NaF tubes. Immediately upon withdrawal, blood was divided into two portions; one portion was centrifuged immediately after collection (10 minutes, $\left.4^{\circ} \mathrm{C}, 3500 \mathrm{rpm}\right)$ and the supernatant was stored as plasma and a second portion was stored as whole blood. At $0,30,60,90$ and 120 minutes, samples were analyzed for the concentration of GO, MGO and 3-DG in plasma and whole blood (figure 6.2 and 6.3). Additionally, plasma was prepared from whole blood which was stored for $0,30,60,90$ and 120 minutes at room temperature, and analyzed for the concentration of GO, M GO and 3-DG in plasma (figure 6.4). Furthermore, the effect of storage on the stability of GO, MGO and 3-DG concentrations was investigated, in plasma and whole blood specimens, with or without immediate precipitation of proteins with PCA.

\section{Sample preparation}

Whole blood was collected in sterile EDTA or EDTA $+\mathrm{NaF}$ tubes and prepared and/or stored as described in the different experiments. Before analysis, plasma samples where thawed (if needed) and mixed thoroughly. Twenty-five $\mu \mathrm{l}$ plasma was mixed with $75 \mu \mathrm{L}$ OPD (10 mg OPD in $10 \mathrm{~mL} 1.6 \mathrm{~mol} / \mathrm{L}$ perchloric acid) in an eppendorf cup. After an overnight $(20 \mathrm{~h})$ reaction at room temperature and shielded from light, $10 \mu \mathrm{l}$ of internal standard solution was added. Samples were mixed and subsequently centrifuged for 20 minutes at $14000 \mathrm{rpm}$ at a temperature of $4^{\circ} \mathrm{C}$. Ten $\mu$ l was injected for UPLC/M SM S analysis. 


\section{UPLC tandem MS}

Derivatized GO, MGO and 3-DG were analyzed by ultra-performance liquid chromatography (Acquity UPLC, Waters, Milford, USA) and detected in ESI positive multiple reaction monitoring (MRM) mode using a Xevo TQ MS (Waters, Milford, USA). Derivatives were separated on a reversed-phase $\mathrm{C} 18$ column (Acquity UPLC BEH C18, $50 \mathrm{x}$ $2.1 \mathrm{~mm}, 1.7 \mu \mathrm{m}$ ) with a binary gradient of $5 \mathrm{mmol} / \mathrm{L}$ formic acid (solvent $A$ ) and acetonitril (solvent $B$ ) at a flow rate of $800 \mu \mathrm{l} / \mathrm{min}$ (split-ratio: waste/tandem MS=1/1). A linear gradient was started at $99 \%$ solvent $A$, wich was changed within 4 minutes to $75 \%$ solvent A. After cleaning the column with $50 \%$ solvent B during 2 minutes, the column was equilibrated for 2 minutes at the initial conditions. The injection volume was $10 \mu \mathrm{L}$ and column temperature was set at $30^{\circ} \mathrm{C}$. Quantification of GO, MGO and 3-DG was performed by calculating the peak area ratio of each unlabeled peak area to the corresponding internal standard peak area. The M RM transitions for GO, MGO and 3-DG were respectively $131.1>77.1,145.1>77.1$ and $235.1>171.1$. The MRM transitions for the internal standards $\left[{ }^{2} \mathrm{H}_{4}\right]-\mathrm{GO},\left[{ }^{2} \mathrm{H}_{4}\right]-\mathrm{MGO}$, and $\left[{ }^{2} \mathrm{H}_{4}\right]-3 D G$ were respectively $135.1>81.1$, $149.1>81.1$ and $239.1>175.1$. Electrospray ionization was done at a capillary voltage of $0.5 \mathrm{kV}$ a source temperature of $150^{\circ} \mathrm{C}$ and a desolvation temperature of $600^{\circ} \mathrm{C}$. For qualitative and quantitative analysis Masslynx software (V4.1, SCN 644, Waters, Milford, USA) was used.

\section{Method validation}

Linearity was determined by adding standard solution of GO, MGO and 3-DG to water and to 6 different plasma samples. A six-point calibration curve was prepared for GO (1458-0 nmol/L), M GO (1083-0 nmol/L) and 3-DG (2320-0 nmol/L). The peak area ratio of GO, MGO and 3-DG multiplied by the concentration of each corresponding internal standard were plotted as a function of the concentration. For the evaluation of the interand intra-assay variation a pooled EDTA plasma sample was analyzed on 10 different days (inter-assay) and 10 times on the same day (intra-assay). Recovery (\%) was tested by adding standard solution of GO, M GO and 3-DG at two different concentration levels (729 and 182 for GO, 542 and 135 for MGO, 1475 and 369 for 3-DG nmol/L) to 6 different plasma samples. Stability of GO, MGO and 3-DG derivatives was tested by replicate injections, every 4 hours, in a pooled EDTA plasma sample. Plasma concentrations were corrected for possible formation of GO, MGO and 3-DG during storage in the autoinjector. 


\section{Subjects}

Diabetic individuals and non-diabetic controls were randomly selected from the Cohort study of Diabetes and Atherosclerosis Maastricht (CODAM). GO, MGO and 3-DG measurements were performed in EDTA plasma. The characteristics of these subjects have been described in detail elsewhere 19. In short, the control group ( $n=10 ; 9$ female) were non-diabetic controls of $58.4 \pm 10.0$ years old and the second group were patients with type 2 diabetes (T2DM) ( $n=10 ; 2$ female) and were $65.3 \pm 6.0$ years old. After an overnight fast, plasma glucose concentrations (mmol/L) were measured with a hexokinase glucose- 6 phosphate dehydrogenase method (ABX Diagnostics, M ontpellier, France). Plasma glucose concentrations were $4.5 \pm 0.1 \mathrm{mmol} / \mathrm{L}$ in non-diabetic controls and $10.2 \pm 0.3 \mathrm{mmol} / \mathrm{L}$ in patients with T2DM.

\section{Statistical Analysis}

The method validation data and patient study data were expressed as mean \pm standard error of the mean (SEM). To detect group differences we applied the Mann-Whitney U t-test. P-value $\varangle .05$ was considered statistically significant.

\section{Results}

\section{UPLC tandem MS analysis}

A representative chromatogram of a standard in water and an EDTA pool plasma sample is shown in figure 6.1A and 6.1B. Retention times of GO, MGO and 3-DG were 2.09, 2.63 and 1.67 minutes, respectively and the corresponding stable isotopes (Figure 6.1C) eluted at $2.06,2.60$ and 1.65 minutes, respectively.

\section{Method validation}

Calibration curves for GO, MGO and 3-DG were linear over the concentration ranges ( $r 2>0.99)$, as described in the method section, in both water and plasma matrix. Mean slope (response factor, Rf) for GO, MGO and 3-DG tested in 6 different plasma samples were $1.036(\mathrm{CV}, 7.5 \%), 0.850(\mathrm{CV}, 8.2 \%)$ and $0.800(\mathrm{CV}, 5.6 \%)$, respectively. M ean Rf for GO, M GO and 3-DG tested in water (analyzed on 3 different days) were 1.175 (CV, 1.5\%), $0.897(\mathrm{CV}, 2.0 \%)$ and 1.011 (CV, 0.9\%), respectively.

Inter-assay variation as determined by replicate analysis of a pooled EDTA plasma sample on 10 different days was for GO, M GO and 3-DG $14.3 \%, 7.3 \%$ and $12.0 \%$, respectively. Intra-assay variation as determined by replicate analysis $(n=10)$ of a pooled EDTA plasma 
sample on one day was for GO, M GO and 3-DG $4.3 \%, 2.9 \%$ and $2.4 \%$, respectively. The lower limits of quantification $(s / N=6$ ) on column for $G 0, M G O$ and 3-DG were 200, 17, and $10 \mathrm{fmol}$, corresponding to a concentration of 100, 9 and $5 \mathrm{nmol} / \mathrm{L}$, respectively. Mean recovery, as tested in 6 different plasma samples at two different concentration levels, was shown in table 6.1.

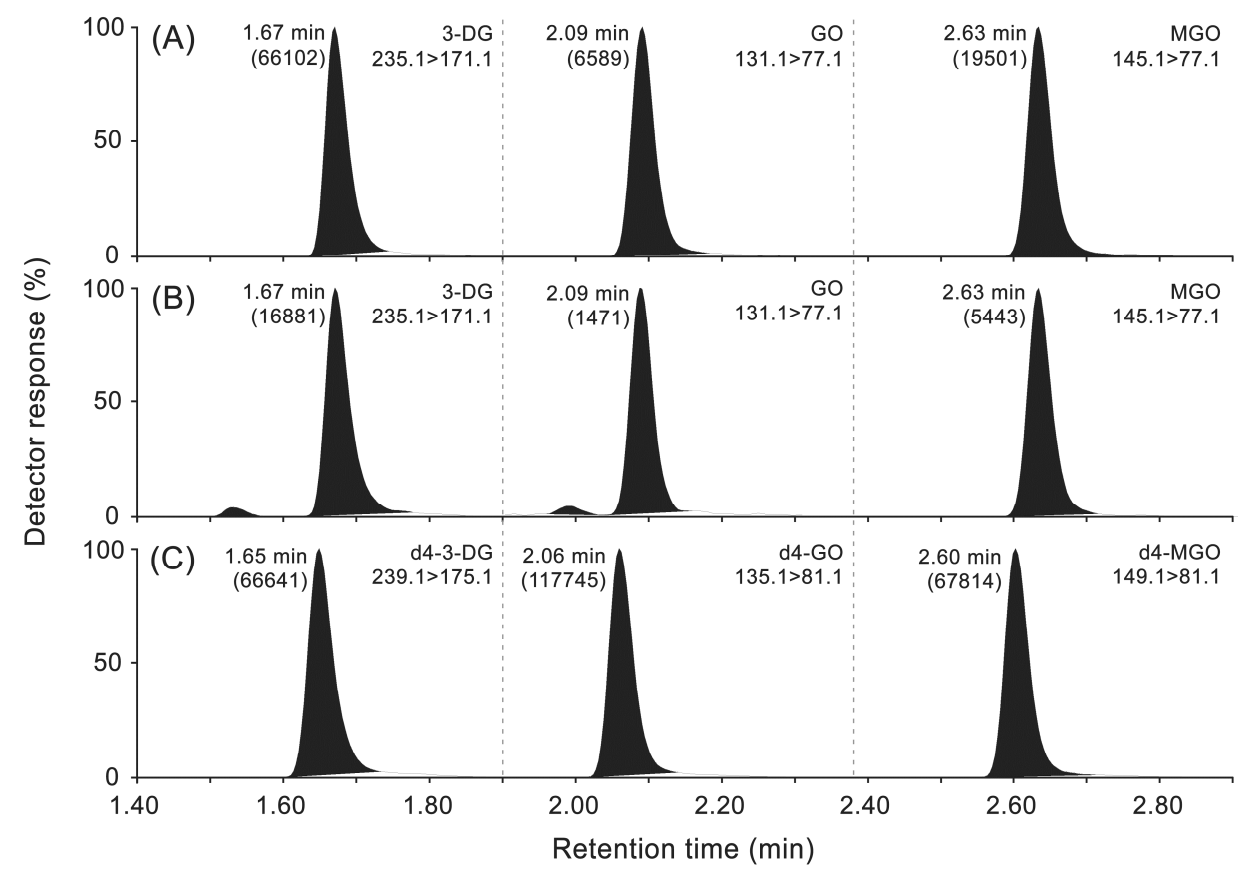

Figure 6.1 Representative chromatogram of a standard (A) of GO, MGO and 3-DG, an EDTA pooled plasma sample (B) and an internal standard (C) of $\left[{ }^{2} \mathrm{H}_{4}\right]-\mathrm{GO},\left[{ }^{2} \mathrm{H}_{4}\right]-\mathrm{M} \mathrm{GO}$ and $\left[{ }^{2} \mathrm{H}_{4}\right]-3 D G$.

Table 6.1 Recovery of GO, M GO and 3DG as tested in 6 different (A to F) plasma samples. Recovery was tested by adding GO, M GO and 3-DG standard, at two different concentration levels, to 6 different plasma samples.

\begin{tabular}{|c|c|c|c|c|c|c|c|c|c|}
\hline & $\begin{array}{l}\text { Added } \\
\text { (nmol/ L) }\end{array}$ & A (\%) & B (\%) & C (\%) & D (\%) & E (\%) & F (\%) & Mean & SD \\
\hline GO high & 729 & 102.6 & 97.2 & 99.6 & 108.8 & 96.6 & 102.3 & 101.2 & 4.5 \\
\hline$\underline{\text { GO low }}$ & 182 & 101.8 & 104.3 & 99.3 & 132.2 & 101.2 & 83.5 & 103.7 & 15.8 \\
\hline MGO high & 541 & 103.5 & 89.8 & 98.8 & 101.8 & 104.0 & 98.9 & 99.5 & 5.2 \\
\hline MGO low & 135 & 108.7 & 80.1 & 95.9 & 99.7 & 108.3 & 77.5 & 95.0 & 13.5 \\
\hline 3-DG high & 1475 & 110.4 & 104.5 & 101.9 & 110.2 & 80.7 & 81.8 & 98.3 & 13.6 \\
\hline 3-DG low & 369 & 126.3 & 113.4 & 99.5 & 119.5 & 86.3 & 72.4 & 102.9 & 20.7 \\
\hline
\end{tabular}




\section{Evaluation of blood specimen}

\section{Stability of GO, M GO and 3-DG in whole blood}

In figure 6.2 the stability of GO, MGO and 3-DG in whole blood is shown. When proteins in blood, collected in EDTA or EDTA + NaF tubes, were directly precipitated with PCA, the concentration of GO, MGO and 3-DG were stable for at least 120 minutes. Without immediate precipitation, the concentration of $G O$ and $M G O$ in blood increased over time in EDTA + NaF tubes and MGO in blood was also increased in EDTA tubes. A slightly decrease of 3-DG in time was observed in both EDTA + NaF and EDTA tubes.
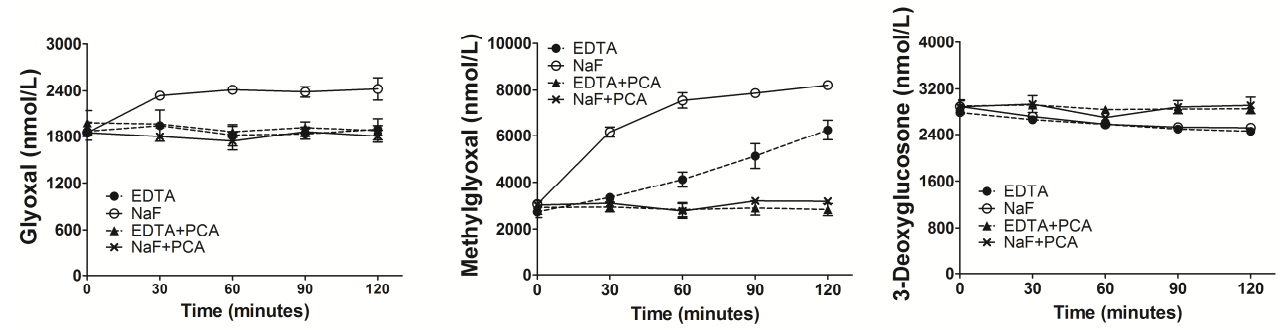

Figure 6.2 Stability of GO, M GO and 3-DG in whole blood (EDTA or EDTA + NaF) after immediate precipitation with PCA or whole blood processed after standing at room temperature for the indicated time followed by precipitation. Data presented as mean \pm SEM .

\section{Stability of GO, MGO and 3-DG in plasma (stored as whole blood)}

In figure 6.3 the stability of GO, M GO and 3-DG in plasma stored as whole blood is shown. The concentration of GO was at least stable for 120 minutes in EDTA + NaF tubes, without the need for immediate centrifugation and precipitation. In contrast, the concentration of GO decreased over time in EDTA tubes. M GO was at least stable for 120 minutes in EDTA tubes, without the need for immediate centrifugation and precipitation with PCA, but increased over time in EDTA+NaF tubes. In both EDTA+NaF and EDTA tubes a decrease of 3-DG was observed.
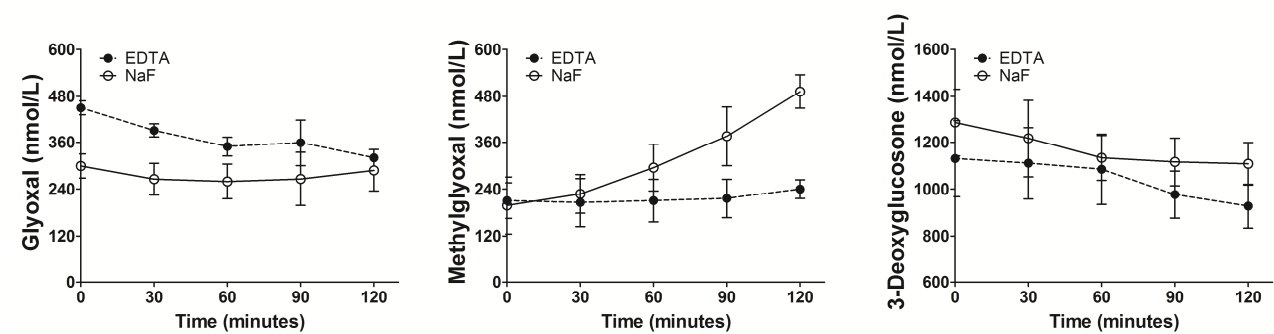

Figure 6.3 Stability of GO, M GO and 3-DG in plasma (stored as whole blood at room temperature, EDTA vs EDTA $+\mathrm{NaF})$. Data presented as mean \pm SEM. 


\section{Stability of GO, M GO and 3-DG in plasma}

To study the stability of GO, M GO and 3-DG in plasma, blood specimen were immediately centrifuged after withdrawal. When the protein fraction in plasma was immediately precipitated with PCA, the three oxoaldehydes were stable for at least 120 minutes in EDTA plasma (figure 6.4). Without immediate precipitation with PCA, MGO remained stable for 120 minutes, but a decrease was observed for $G 0$ and 3-DG after 60 minutes.
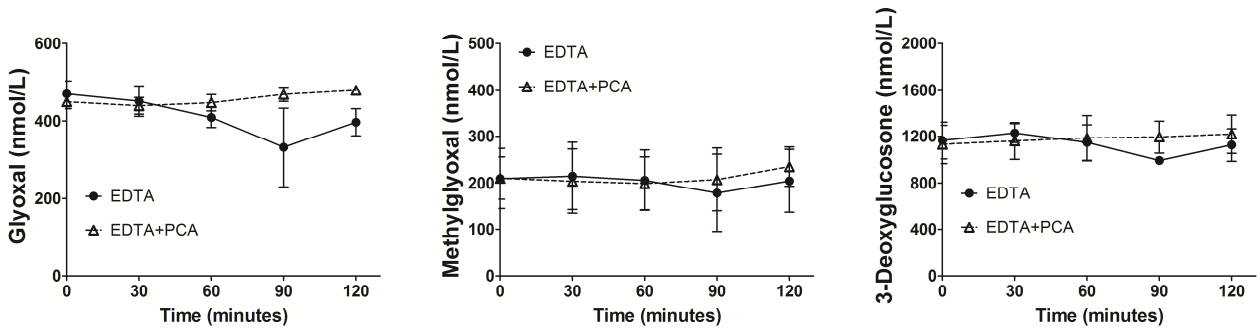

Figure 6.4 Stability of GO, M GO and 3-DG in plasma (EDTA) after immediate precipitation with PCA or without immediate precipitation. Data presented as mean \pm SEM .

\section{Concentration of GO, M GO and 3-DG in non-diabetic controls vs patients with type 2 diabetes}

When compared to healthy controls, EDTA plasma from patients with type 2 diabetes showed significantly increased concentrations of MGO (277 \pm 9 vs $212 \pm 8 \mathrm{nmol} / \mathrm{L})$ and 3-DG (2217 \pm 81 vs $1046 \pm 37 \mathrm{nmol} / \mathrm{L}$ ) (figure 6.5). Plasma G0 concentration was also increased in T2DM vs non-diabetic controls ( $514 \pm 49$ vs $406 \pm 26 \mathrm{nmol} / \mathrm{L}$ ), although not significant.
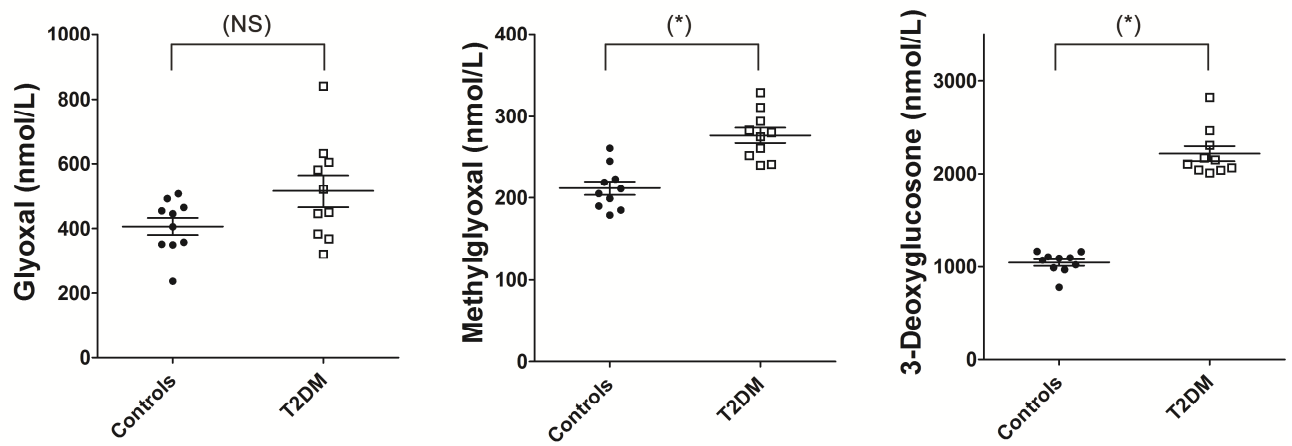

Figure 6.5 Plasma GO, MGO and 3-DG concentrations as measured in non-diabetic controls vs T2DM. Data presented as mean \pm SEM ( $*=p<0.05$, NS=not significant). 


\section{Discussion}

We describe here a rapid, sensitive, precise and accurate method based on stable isotope dilution UPLC MS/MS for the quantification of GO, M GO and 3-DG in blood and plasma. With the use of this method, we found that plasma levels of GO, MGO and 3-DG were increased in T2DM as compared with non-diabetic controls.

Many techniques have been used to quantify GO, MGO and 3-DG. However, several disadvantages of these techniques were noticed, such as large sample volume $e^{1,8,11,13}$, elaborate LLE or SPE steps ${ }^{1,2,8,11,16,17}$ and long run-times ${ }^{2,12,15}$. Moreover, the use of nonstable isotope internal standards $s^{1,5,7,8,17,20}$ may cause large uncertainties in quantifying analytes using electrospray ionization UPLC M S/MS due to matrix effects ${ }^{7,8}$. So far, the absolute concentrations of GO, M GO and 3-DG differ between studies, ${ }^{5,6,13,21}$, most likely due to different sample preparation (e.g. deproteinization, duration of derivatisation and calibration) and/or sample handling during storage and the method of detection. In the current study we indeed found large differences in plasma $\alpha$-oxoaldehyde levels as a result of sample handling during storage.

We present a rapid, sensitive and accurate method for quantifying GO, MGO and 3-DG in plasma. With a sample volume of only $25 \mu \mathrm{l}$ and a run-to-run time of 8 minutes this method is very suitable for analyzing GO, MGO and 3-DG in large cohort studies. Furthermore, the use of stable isotope internal standards is an improvement over mass spectrometry methods described in literature so far.

GO, MGO and 3DG are very reactive $\alpha$-0xoaldehydes which can react very fast with proteins to AGEs. In addition to the reaction of these $\alpha$-oxoaldehydes with proteins, these $\alpha$-oxoaldehydes can also be formed during sample preparation. Therefore, variations in sample treatment and the interval between the blood draw and sample analysis can substantially influence the free concentrations of these $\alpha$-oxoaldehydes. We found a progressive increase of $\mathrm{MGO}$ and $\mathrm{GO}$ in blood drawn in $\mathrm{NaF}$ tubes as stored at room temperature. The corresponding plasma also showed an increase of the MGO concentration in time. $\mathrm{NaF}$ is used in blood glucose measurements as an inhibitor of glycolysis. Fluoride acts primarily by inhibiting enolase in the glycolytic pathway ${ }^{22}$ and prevents the formation of pyruvate out of glucose. Since $\alpha$-oxoaldehydes can be formed during glycolysis ${ }^{21}$, we anticipated $\alpha$-oxoaldehydes to remain constant in time during storage in $\mathrm{NaF}$ tubes. However, this was not the case for MGO and GO. Foregoing explanation for this increase of $\mathrm{GO}$ and $\mathrm{MGO}$ by NaF is that the inhibition of enolase may lead to the accumulation of precursors for $G O$ and MGO, i.e. dihydroxyacetone phosphate and glyceraldehyde-3-phosphate, which may lead to an increase of GO and M GO during storage in EDTA + NaF tubes. Indeed, this increase in M GO was not noted in EDTA tubes and supported that the effect of fluoride on MGO is due to blockage of the glycolysis at the level of enolase. Because of the above mentioned effect, EDTA $+\mathrm{NaF}$ tubes should be avoided and EDTA tubes are preferable. 
For the quantification of $\alpha$-oxoaldehydes in blood, we have found that precipitation of proteins by PCA within 30 minutes after blood withdrawal is necessary. Under this condition, no change in the concentration of M GO and GO and 3-DG was noticed.

For the quantification of $\alpha$-oxoaldehydes in EDTA plasma, we found that the concentration of $\alpha$-oxoaldehyde in plasma remained stable for at least 60 minutes without protein precipitation. However, a direct precipitation of plasma proteins by PCA stabilized $\alpha$-oxoaldehyde levels for at least 2 hours when stored at room temperature and this condition is preferable. Taken together, for quantification of $\alpha$-oxoaldehydes in blood and plasma, EDTA tubes and a direct PCA precipitation is recommended.

When using EDTA plasma, we found increased levels of $\alpha$-oxoaldehydes in T2DM as compared to non-diabetic controls which is in reasonable agreement with data described in literature ${ }^{5,6,13,21}$. Nevertheless, absolute concentrations differ between studies; Plasma concentrations as measured in non-diabetic controls varied for MGO from 96 to $652 \mathrm{nmol} / \mathrm{L}^{1,5,6,13,21}$ and for GO from 328 to $1154 \mathrm{nmol} / \mathrm{L}^{1,5}$ and the concentration of 3-DG was reported to be $160 \mathrm{nmol} / \mathrm{L}^{5}$. In our measurements we found plasma concentrations for MGO, GO and 3-DG of $212 \mathrm{nmol} / \mathrm{L}, 406 \mathrm{nmol} / \mathrm{L}$ and $1046 \mathrm{nmol} / \mathrm{L}$, respectively. The concentration differences of GO, MGO and 3-DG, as found in different studies, are most likely due to sample preparation and sample handling during storage.

In conclusion, for the quantification of $\alpha$-oxoaldehydes in blood and plasma samples, we recommend EDTA as anticoagulant, a direct protein precipitation of the samples and the use of stable isotope dilution UPLCMS/MS as the method of detection. 


\section{References}

1

2

3

4

5

6

7

8

Han, Y. et al. Plasma methylglyoxal and glyoxal are elevated and related to early membrane alteration in young, complication-free patients with Type 1 diabetes. M ol Cell Biochem 305, 123-131 (2007).

Yamada, H. et al. Increase in 3-deoxyglucosone levels in diabetic rat plasma. Specific in vivo determination of intermediate in advanced M aillard reaction. J Biol Chem 269, 20275-20280 (1994).

Thornalley, P. J., Langborg, A. \& Minhas, H. S. Formation of glyoxal, methylglyoxal and 3deoxyglucosone in the glycation of proteins by glucose. Biochem J 344 Pt 1, 109-116 (1999).

Baynes, J. W. \& Thorpe, S. R. Role of oxidative stress in diabetic complications: a new perspective on an old paradigm. Diabetes 48, 1-9 (1999).

5 Odani, H., Shinzato, T., M atsumoto, Y., Usami, J. \& M aeda, K. Increase in three alpha,beta-dicarbonyl compound levels in human uremic plasma: specific in vivo determination of intermediates in advanced M aillard reaction. Biochem Biophys Res Commun 256, 89-93 (1999).

Lu, J. et al. Increased plasma methylglyoxal level, inflammation, and vascular endothelial dysfunction in diabetic nephropathy. Clin Biochem 44, 307-311 (2011).

Ogawa, S. et al. Methylglyoxal is a predictor in type 2 diabetic patients of intima-media thickening and elevation of blood pressure. Hypertension 56, 471-476 (2010).

Nakayama, K. et al. Plasma alpha-oxoaldehyde levels in diabetic and nondiabetic chronic kidney disease patients. Am J Nephrol 28, 871-878 (2008).

Nin, J. W. et al. Higher plasma levels of advanced glycation end products are associated with incident cardiovascular disease and all-cause mortality in type 1 diabetes: a 12-year follow-up study. Diabetes Care 34, 442-447 (2011).

Srikanth, V. et al. Advanced glycation endproducts and their receptor RAGE in Alzheimer's disease. Neurobiol Aging 32, 763-777 (2011).

McLellan, A. C., Phillips, S. A. \& Thornalley, P. J. The assay of methylglyoxal in biological systems by derivatization with 1,2-diamino-4,5-dimethoxybenzene. Anal Biochem 206, 17-23 (1992).

Mittelmaier, S., Funfrocken, M., Fenn, D., Fichert, T. \& Pischetsrieder, M. Identification and quantification of the glucose degradation product glucosone in peritoneal dialysis fluids by HPLC/DAD/M SM S. J Chromatogr B Analyt Technol Biomed Life Sci 878, 877-882 (2010).

Nemet, I., Varga-Defterdarovic, L. \& Turk, Z. Preparation and quantification of methylglyoxal in human plasma using reverse-phase high-performance liquid chromatography. Clin Biochem 37, 875881 (2004).

Neng, N. R., Cordeiro, C. A. A., Freire, A. P. \& Nogueira, J. M. F. Determination of glyoxal and methylglyoxal in environmental and biological matrices by stir bar sorptive extraction with in-situ derivatization. J Chromatogr A 1169, 47-52 (2007).

Dhar, A., Desai, K., Liu, J. \& Wu, L. Methylglyoxal, protein binding and biological samples: are we getting the true measure? J Chromatogr B Analyt Technol Biomed Life Sci 877, 1093-1100 (2009).

6 Mittelmaier, S., Funfrocken, M., Fenn, D., Berlich, R. \& Pischetsrieder, M. Quantification of the six major alpha-dicarbonyl contaminants in peritoneal dialysis fluids by UHPLC/DAD/M SM S. Anal Bioanal Chem 401, 1183-1193 (2011). 
17 Randell, E. W., Vasdev, S. \& Gill, V. Measurement of methylglyoxal in rat tissues by electrospray ionization mass spectrometry and liquid chromatography. J Pharmacol Toxicol M ethods 51, 153-157 (2005).

18 Kampf, C. J., Bonn, B. \& Hoffmann, T. Development and validation of a selective HPLC-ESI-M S/MS method for the quantification of glyoxal and methylglyoxal in atmospheric aerosols (PM 2.5). Anal Bioanal Chem 401, 3115-3124 (2011).

19 Jacobs, M. et al. Low-grade inflammation can partly explain the association between the metabolic syndrome and either coronary artery disease or severity of peripheral arterial disease: the CODAM study. Eur J Clin Invest 39, 437-444 (2009).

20 Beisswenger, P. J., Howell, S. K., Touchette, A. D., Lal, S. \& Szwergold, B. S. Metformin reduces systemic methylglyoxal levels in type 2 diabetes. Diabetes 48, 198-202 (1999).

21 Fleming, T. et al. Is diabetes an acquired disorder of reactive glucose metabolites and their intermediates? Diabetologia 55, 1151-1155, doi:10.1007/s00125-012-2452-1 (2012).

22 Mikesh, L. M. \& Bruns, D. E. Stabilization of glucose in blood specimens: mechanism of delay in fluoride inhibition of glycolysis. Clin Chem 54, 930-932 (2008). 


\section{DOST-GLUCOSE LOAD PLASMA}

$\alpha$-dicarbonyl concentrations ARE INCREASED IN INDIVIDUALS WIIH impaired glucose metabolism and type 2 diabetes:

THE CODAM STUDY

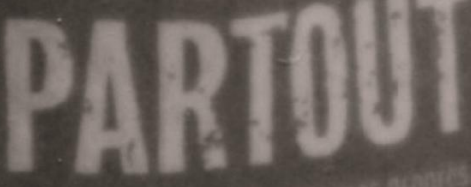

Aidez nous a gord

ist lovites

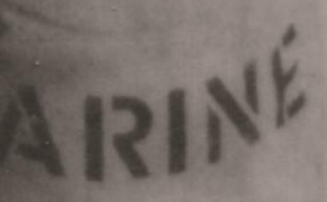




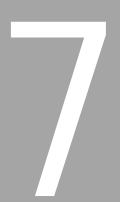

Post-glucose load plasma $\alpha$-dicarbonyl concentrations are increased in individuals with impaired glucose metabolism and type 2 diabetes: The CODAM study

Dionne E.M. Maessen, Nordin M.J. Hanssen, Jean LJ.M. Scheijen, Carla J. van der Kallen, M arleen M.J. van Greevenbroek, Coen D.A. Stehouwer and Casper G. Schalkwijk

Diabetes Care 38; 913-920 (2015) 


\section{Abstract}

\section{Objective:}

There is increasing evidence that postprandial glucose excursions play an important role in the development of vascular complications. The underlying mechanism is unknown, but glucose-derived formation of reactive $\alpha$-dicarbonyl compounds may explain why acute hyperglycemia leads to increased risk for diabetic complications. In the current study, we investigated whether $\alpha$-dicarbonyls are increased after a glucose load in individuals without and with impaired glucose metabolism (IGM) and type 2 diabetes.

\section{Research Design and Methods:}

Cross-sectional, linear analyses were performed in the Cohort study on Diabetes and Atherosclerosis Maastricht (CODAM, $n=574,61 \%$ men, 60 years old). Individuals with normal glucose metabolism ( $n=279)$, IGM $(n=120)$ and type 2 diabetes $(n=92)$ who had complete data on an oral glucose tolerance test (OGTT) and were not on insulin treatment were included in the study population. Plasma $\alpha$-dicarbonyl (methylglyoxal (MGO), glyoxal (GO) and 3-deoxyglucosone (3-DG)) levels were measured in the fasting state and in samples of the OGTT by UPLC-MS/MS.

\section{Results:}

The presence of both IGM and type 2 diabetes was significantly associated with higher $\alpha$-dicarbonyl incremental area under the curves (iAUC), as calculated from the OGTT (for IGM: MGO $\beta=0.190,95 \% \mathrm{Cl}=0.106-0.274 ; \mathrm{GO} \quad \beta=0.287,95 \% \mathrm{Cl}=0.172-0.401 ; 3-\mathrm{DG}$ $\beta=0.285,95 \% \mathrm{Cl}=0.221-0.349$; for type 2 diabetes: $M G O \quad \beta=0.293,95 \% \mathrm{Cl}=0.180-0.405$; GO $\beta=0.536,95 \% \mathrm{Cl}=0.382-0.689 ; 3-\mathrm{DG} \beta=0.542,95 \% \mathrm{Cl}=0.456-0.628)$. Adjustment for glucose iAUC attenuated these associations. iAUCs of the $\alpha$-dicarbonyls correlated highly with glucose iAUC, but not with fasting glucose levels and $\mathrm{HbAlC}$.

\section{Conclusions:}

The increased levels of $\alpha$-dicarbonyls during an OGTT in individuals with IGM and type 2 diabetes underline the potential importance of $\alpha$-dicarbonyl stress as a candidate to explain the increased risk of diabetic complications in individuals with postprandial hyperglycemia. 


\section{Introduction}

Type 2 diabetes and impaired glucose metabolism (IGM) are associated with the development of microvascular complications and cardiovascular disease (CVD) ${ }^{1}$. Prospective studies in diabetes have shown a strong correlation between mean glucose levels, as reflected by $\mathrm{HbA1C}$, and diabetic complications. However, in recent years, postprandial glucose excursions, rather than fasting glucose concentrations or mean glucose levels, have increasingly been recognized as a contributing factor to the increased risk of vascular complications ${ }^{2,3}$. Controlled clinical trials, such as the Diabetes Control and Complications Trial (DCCT) in type 1 diabetes ${ }^{4}$, and the United Kingdom Prospective Diabetes Study (UKPDS) in type 2 diabetes ${ }^{5}$, have established that intensive therapies which reduce $\mathrm{HbAlC}$ levels, reduce and delay the development and progression of diabetes-related long-term vascular complications. Post-trial analyses of the DCCT revealed that post-meal glucose levels contribute more to $\mathrm{HbAlC}_{\neg}$ than fasting plasma levels ${ }^{6}$, suggesting that postprandial hyperglycemic spikes may accelerate the onset of diabetic complications. Moreover, many epidemiological data support this concept, showing that glucose levels after an oral glucose tolerance test (OGTT) are an independent risk factor for CVD, while fasting glucose levels are not or less $50^{2,7-11}$. In addition, postprandial glucose in type 2 diabetes predicts myocardial infarctions ${ }^{12}$ and is a risk factor for CVD ${ }^{13}$. However, it is largely unknown how these postprandial glucose peaks cause the increased risk for diabetic complications. A possible mechanism through which they have a more damaging effect than high fasting or mean glucose levels might be via the formation of $\alpha$-dicarbonyls.

The $\alpha$-dicarbonyls, methylglyoxal (M GO), glyoxal (GO) and 3-deoxyglucoseone (3-DG), are mainly formed as glycolytic intermediates by metabolic conversion of glucose. We and others have shown the importance of $\alpha$-dicarbonyls in the development of nephropathy ${ }^{14,15}$, retinopathy ${ }^{16}$ and neuropathy ${ }^{17,18}$, and in macrovascular complications ${ }^{19}$, using rodent models of diabetes.

We hypothesized that the postprandial glucose concentrations, rather than fasting plasma glucose, determine $\alpha$-dicarbonyl levels. Although Beisswenger et al. already demonstrated, in a small study of 21 individuals with type 1 diabetes, that both MGO and 3-DG plasma levels increase after postprandial glycemic excursions ${ }^{20}$, the question whether $\alpha$-dicarbonyl levels are elevated in individuals with impaired glucose metabolism (IGM) and type 2 diabetes remains unanswered.

In the present study, we investigated, in a large cohort study, whether plasma levels of $\alpha$-dicarbonyls are elevated in individuals with IGM and type 2 diabetes. Both fasting levels and incremental area under the curves (iAUC) obtained from an OGTT were analyzed. 


\section{Research design and methods}

\section{Study population}

The present study comprises participants from the Cohort study on Diabetes and Atherosclerosis M aastricht (CODAM), which included 574 participants who were selected from the general population as described in detail elsewhere ${ }^{21}$. The CODAM study was designed to investigate cardiovascular and metabolic function, and was enriched for IGM status, as described previously ${ }^{22}$. Current main analyses were performed on 491 participants who underwent a full OGT and were not on insulin treatment. The study was approved by the local Medical Ethical Committee of Maastricht University Medical Centre and all participants gave written informed consent.

\section{Definition of glucose metabolism status}

The glucose tolerance status of the participants was determined by an OGT. After an overnight fasting period (10-12 hours), study participants underwent a standard $75 \mathrm{~g}$ OGTT (82 g dextrose monohydrate, Avebe, The Netherlands) and venous blood was obtained prior to, and at 30,60 and 120 minutes after the glucose load. Fasting and postload plasma glucose concentrations (in $\mathrm{mmol} / \mathrm{L}$ ) were measured with a hexokinase glucose-6 phosphate dehydrogenase method (ABX Diagnosis, Montpellier, France). Fasting and $2 \mathrm{~h}$ post-load glucose concentrations were used to classify the study participants' glucose metabolism status (GMS) according to the World Health Organization (WHO) criteria. Briefly, individuals were classified as having normal glucose metabolism (NGM) when they had normal fasting $(<6.1 \mathrm{mmol} / \mathrm{L})$ and $2 \mathrm{~h}$ post-load $(<7.8 \mathrm{mmol} / \mathrm{L})$ glucose concentrations. Individuals with impaired fasting glucose (6.1$7.0 \mathrm{mmol} / \mathrm{L})$, impaired $2 \mathrm{~h}$ post-load glucose levels $(7.8-11.1 \mathrm{mmol} / \mathrm{L})$, or both were classified as having IGM. When individuals had high fasting plasma glucose levels $(>7.0 \mathrm{mmol} / \mathrm{L})$ and/or high $2 \mathrm{~h}$ post-load glucose levels (>11.1 mmol/L), or when they used glucose-lowering medication or insulin, they were classified as having type 2 diabetes ${ }^{22}$. Individuals with known type 2 diabetes, or those with fasting glucose levels $>8.5 \mathrm{mmol} / \mathrm{L}$ were excluded from undergoing an OGTT.

\section{M easurements of plasma $\alpha$-dicarbonyls}

Plasma levels of $\alpha$-dicarbonyls were measured in EDTA plasma samples from the OGTT at baseline and 30,60 and 120 minutes after the glucose load. Blood samples were collected in EDTA tubes which were stored on ice prior to blood sampling to ensure rapid cooling of the blood. 
After withdrawal of the blood sample, tubes were stored on ice immediately, and were spun within two hours at $3000 \mathrm{rpm}, 4^{\circ} \mathrm{C}$. Plasma samples have been stored at $-80^{\circ} \mathrm{C}$ until analysis. Reversed phase ultra-performance liquid chromatography tandem mass spectrometry (UPLC-MS/MS) was used to analyze the plasma samples for M GO, GO and 3-DG, as described previously ${ }^{23}$. The inter-assay variations for M GO, GO and 3-DG were $4.3,5.1$ and $2.2 \%$, respectively. Current analyses were performed with fasting $\alpha$-dicarbonyl levels and OGTT iAUCs.

\section{Calculation of the OGTT incremental area under the curve}

The area under the curve (AUC) for the OGTT levels of the $\alpha$-dicarbonyls and glucose was calculated according to the trapezoidal method ${ }^{24}$, where baseline (fasting) levels were subtracted from each individual data point to specify the post-glucose load increases. These data are referred to as the iAUC.

\section{Covariates}

Waist circumference and prior CVD were assessed as described previously ${ }^{25}$. Questionnaires were used to asses smoking behavior (never, ever or current smoker) and use of medication (lipid-, glucose- and blood pressure-lowering medication). Plasma creatinine levels were measured with the Jaffé diagnostic test (Roche Diagnostics, Mannheim, Germany) and the estimated glomerular filtration rate (eGFR) was calculated using the Chronic Kidney Disease Epidemiology Collaboration (CKD-EPI) equation ${ }^{26}$. Systolic blood pressures were measured in the brachial arteries and twice in both the tibialis posterior and dorsalis pedis arteries of the lower extremities with a standard Doppler device (Mini Dopplex D900, Huntleigh Diagnostics Ltd, Harmelen, The Netherlands) ${ }^{27}$. Total and HDL-cholesterol and triglycerides were measured in EDTA plasma by enzymatic methods (Roche Diagnostics, M annheim, Germany) ${ }^{27}$.

\section{Statistical analyses}

The general characteristics of the study population were compared across the three groups of GMS. Continuous variables were analyzed with one-way ANOVA and discrete variables were analyzed with chi-square tests. Fasting levels and iAUCs from the OGTT were normally distributed for all $\alpha$-dicarbonyls. To increase statistical power, missing values on $\mathrm{HbAlC}$ ( $3.7 \%$ missings), eGFR ( $0.8 \%$ missings) and $\mathrm{BMI}(0.2 \%$ missings) were imputed by creating 5 datasets using multiple imputation. Skewed variables (fasting plasma glucose, fasting plasma insulin, HOM A-IR and triglycerides) were loge transformed prior to analyses. 
Two-way repeated measures ANOVA with Bonferroni correction was used to compare the curves of the $\alpha$-dicarbonyls during the OGTT between NGM, IGM and type 2 diabetes over time. Fasting levels and iAUCs of the $\alpha$-dicarbonyls were compared between the groups using one-way ANOVA with Bonferroni correction. Multiple linear regression analysis was used to investigate potential influence of confounding factors on the association between GMS and $\alpha$-dicarbonyl levels. The main independent variables were GMS, analyzed as dummies for IGM and type 2 diabetes, fasting glucose levels, iAUC glucose and $\mathrm{HbA1C}$, and the main dependent variables were fasting levels and iAUCs of the $\alpha$-dicarbonyls. Data are presented as standardized regression coefficients $(\beta)$. Analyses were initially adjusted for age and sex, and for analyses with fasting glucose levels, iAUC glucose and HbA1C, also for GMS (model 1). Analyses were further adjusted for smoking status, eGFR, loge triglycerides, total-to-HDL cholesterol ratio, waist circumference, prior CVD, systolic blood pressure and use of medication (lipid-, glucoseand blood pressure-lowering medication) (model 2). Finally, analyses for GMS were additionally adjusted for glucose (fasting or iAUC glucose, appropriate to the $\alpha$-dicarbonyl measure), as a potential source of $\alpha$-dicarbonyl formation (model 3 ), and to investigate to which extent iAUCs were dependent on fasting $\alpha$-dicarbonyl levels, model 2 was additionally adjusted for fasting plasma levels of the appropriate $\alpha$-dicarbonyl (model 4). A p-value of $\varangle$.05 was considered statistically significant.

As increased levels of $\alpha$-dicarbonyls can be a result of both increased formation as well as decreased detoxification, we investigated whether individuals with IGM or type 2 diabetes had a decreased capacity to detoxify $\alpha$-dicarbonyls. To test this, we analyzed whether the association between post-load glucose excursions and $\alpha$-dicarbonyl iAUCs differed between individuals with NGM, IGM and type 2 diabetes. To this end, the interaction terms IGM * iAUC glucose and type 2 diabetes*iAUC glucose were added to the linear regression model (adjusted for covariates in model 2). pinteraction $\varangle$.1 was considered statistically significant. Significant $p$-values for interaction would indicate a different detoxification capacity of $\alpha$-dicarbonyls in individuals with IGM or type 2 diabetes, for the same change in glucose concentration.

All statistical analyses were performed with IBM SPSS Statistics Software, version 20 (IBM Corporation, Armonk, New York). 


\section{Results}

General characteristics of the study population stratified according to GM S are shown in table 7.1. Overall, participants with type 2 diabetes were slightly older, had a higher BMI, higher blood pressure, lower HDL-cholesterol and more often CVD and microalbuminuria. Curves of the OGTT in figure 7.1 demonstrate increased levels of glucose and MGO, GO and 3-DG after the glucose load. Plasma glucose levels were approximately 5,000-fold higher compared to the plasma $\alpha$-dicarbonyl levels.

Univariate associations between GMS and both fasting plasma $\alpha$-dicarbonyls and the OGTT iAUC of the plasma $\alpha$-dicarbonyls

When we analyzed the fasting $\alpha$-dicarbonyl levels, we found approximately 1.1-fold higher plasma concentrations of MGO in individuals with type 2 diabetes, but not with IGM (table 7.1, $<<0.001$ ), as compared to NGM. Fasting levels of 3-DG were increased in both individuals with IGM and type 2 diabetes compared to those with NGM (table 7.1, both $p<0.001$ ). GO levels at baseline did not differ between the groups (table 7.1).

When we additionally included individuals with known type 2 diabetes who were not allowed to undergo an OGTT, we observed significantly higher fasting levels of plasma $\alpha$-dicarbonyls, compared to levels from those who did undergo the OGTT (figure 7.1).

A

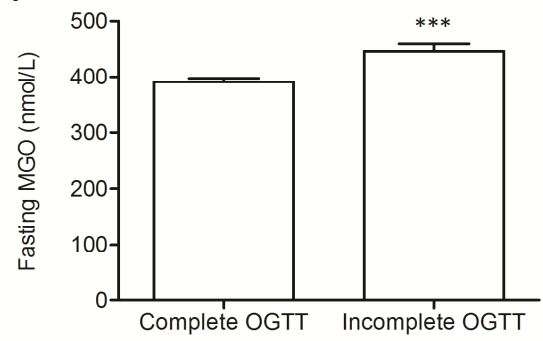

C

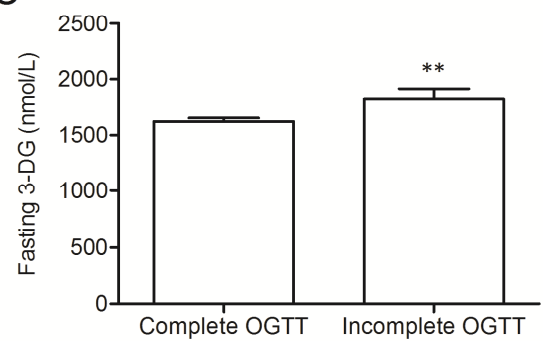

B

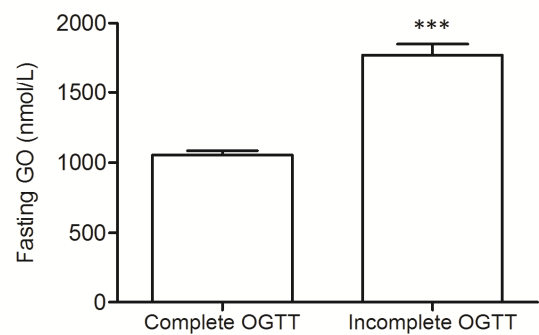

D

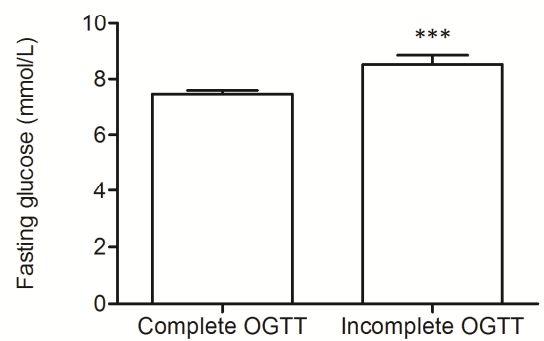

Figure 7.1. Fasting plasma $\alpha$-dicarbonyl levels of individuals without and with a complete OGT. Fasting plasma levels of (A) MGO, (B) GO, (C) 3-DG and (D) glucose in individuals who underwent a complete OGT and individuals who were not able to undergo a complete OGTT. Analyses were performed in all individuals with type 2 diabetes who were not on insulin treatment. Data are shown as means \pm standard error of the means. Differences between the two groups were tested with an independent samples t-test. $* * p \varangle 0.01$ and $* * * p \varangle 0.001$. M GO, methylglyoxal; GO, glyoxal; 3-DG, 3-deoxyglucosone; OGT, oral glucose tolerance test. 
Table 7.1. General characteristics of the CODAM study population stratified according to glucose metabolism status.

\begin{tabular}{|c|c|c|c|c|}
\hline & $\begin{array}{l}\text { NGM } \\
(n=279)\end{array}$ & $\begin{array}{l}\text { IGM } \\
(n=120)\end{array}$ & $\begin{array}{l}\text { Type } 2 \text { diabetes } \\
(n=92)\end{array}$ & $P_{\text {trend }}$ \\
\hline Age (years) & $58.8 \pm 7.4$ & $59.9 \pm 6.7$ & $60.4 \pm 6.2$ & 0.056 \\
\hline Sex ( $\%$ men $)$ & 58.8 & 60.0 & 64.1 & 0.386 \\
\hline $\mathrm{BMI}\left(\mathrm{kg} / \mathrm{m}^{2}\right)$ & $27.5 \pm 3.9$ & $29.0 \pm 4.3$ & $30.1 \pm 4.5$ & $\varangle 0.001$ \\
\hline Waist $(\mathrm{cm})$ & $96.0 \pm 10.9$ & $100.9 \pm 11.9$ & $104.2 \pm 11.5$ & $\varangle 0.001$ \\
\hline $\mathrm{HbA}_{1 \mathrm{C}}(\%)$ & $5.6 \pm 0.4$ & $5.8 \pm 0.4$ & $6.6 \pm 0.9$ & $\varangle 0.001$ \\
\hline $\mathrm{HbA}_{1 \mathrm{C}}(\mathrm{mmol} / \mathrm{mol})$ & $38.0 \pm 4.8$ & $40.3 \pm 4.7$ & $48.4 \pm 9.9$ & $\varangle 0.001$ \\
\hline Fasting plasma M GO (nmol/L) & $350 \pm 71$ & $353 \pm 57$ & $392 \pm 72$ & $\varangle 0.001$ \\
\hline Fasting plasma GO (nmol/L) & $1087 \pm 287$ & $1107 \pm 279$ & $1057 \pm 280$ & 0.442 \\
\hline Fasting plasma 3-DG (nmol/L) & $1102 \pm 156$ & $1191 \pm 136$ & $1619 \pm 300$ & $\varangle 0.001$ \\
\hline Fasting plasma glucose (mmol/L) & $5.3[5.0-5.5]$ & $6.0[5.5-6.3]$ & $7.3[6.9-8.2]$ & $\varangle 0.001$ \\
\hline Fasting plasma insulin (pmol/L) & $52.0[41.0-69.0]$ & $67.0[46.0-100.0]$ & $85.5[56.5-124.8]$ & $\varangle 0.001$ \\
\hline HOM A-IR & $0.98[0.78-1.29]$ & $1.30[0.89-1.91]$ & $1.72[1.14-2.49]$ & $\varangle 0.001$ \\
\hline Glucose-lowering medication (\%) & 0.0 & 2.5 & 33.7 & $\varangle 0.001$ \\
\hline Systolic blood pressure (mmHg) & $135.6 \pm 17.5$ & $143.8 \pm 19.3$ & $147.9 \pm 18.6$ & $\varangle 0.001$ \\
\hline Diastolic blood pressure (mmHg) & $80.1 \pm 8.1$ & $84.1 \pm 9.8$ & $85.4 \pm 10.3$ & $\varangle 0.001$ \\
\hline M ean arterial pressure $(\mathrm{mmHg})$ & $98.6 \pm 10.3$ & $104.0 \pm 12.0$ & $106.2 \pm 11.5$ & $\varangle 0.001$ \\
\hline Anti-hypertensive medication (\%) & 28.3 & 43.3 & 51.1 & $\varangle 0.001$ \\
\hline Prior CVD (\%) & 22.9 & 27.5 & 37.0 & 0.009 \\
\hline Ex-smokers (\%) & 46.6 & 53.3 & 59.8 & 0.072 \\
\hline Current smokers (\%) & 19.7 & 19.2 & 18.5 & 0.791 \\
\hline $\mathrm{eGFR}\left(\mathrm{mL} / \mathrm{min} / 1.73 \mathrm{~m}^{2}\right)$ & $90.8 \pm 11.7$ & $91.5 \pm 11.6$ & $92.3 \pm 13.5$ & 0.310 \\
\hline M icroalbuminuria (\%) & 3.9 & 4.2 & 9.8 & 0.048 \\
\hline Macroalbuminuria (\%) & 0.4 & 0.8 & 2.2 & 0.105 \\
\hline Total cholesterol (mmol/L) & $5.2 \pm 0.9$ & $5.3 \pm 0.9$ & $5.4 \pm 1.1$ & 0.058 \\
\hline HDL cholesterol (mmol/L) & $1.3 \pm 0.4$ & $1.2 \pm 0.3$ & $1.1 \pm 0.3$ & $\varangle 0.001$ \\
\hline LDL cholesterol (mmol/L) & $3.4 \pm 0.9$ & $3.3 \pm 0.9$ & $3.2 \pm 1.1$ & 0.309 \\
\hline Triglycerides (mmol/L) & $1.2[0.9-1.6]$ & $1.6[1.1-2.2]$ & $2.0[1.3-2.6]$ & $\varangle 0.001$ \\
\hline Lipid-lowering medication (\%) & 15.1 & 20.0 & 19.6 & 0.221 \\
\hline
\end{tabular}

Data are presented as means \pm standard deviations, medians (interquartile range), or percentages, as appropriate. 3-DG, 3-deoxyglucosone; CVD, cardiovascular disease; eGFR, estimated glomerular filtration rate; GO, glyoxal; HbA1C, glycated hemoglobin; HDL, high-density lipoprotein; HOMA-IR, homeostasis model assessment insulin resistance; LDL, low-density lipoprotein; MGO, methylglyoxal. Linear trend was tested with one-way ANOVA for continuous variables and with chi-square for discrete variables, as appropriate.

iAUCs for the a-dicarbonyls M GO, GO, and 3-DG were significantly higher (1.6-, 2.3- and 1.8 -fold change, respectively) in participants with IGM (all $p \varangle 0.001$ ) and were even higher (2.1-, 3.4-, and 2.6-fold change, respectively) in those with type 2 diabetes (all $p<0.001$ ) (figure 7.2B, D, and F) compared with NGM. For glucose, both fasting levels and iAUC of the OGT were increased in individuals with IGM and type 2 diabetes (table 7.1 and figure $7.2 \mathrm{H})$ (all $\mathrm{p} \varangle 0.001$ ). 


\section{Multivariate associations between GMS and fasting plasma a-dicarbonyl levels}

The presence of IGM was not associated with higher fasting plasma levels of M GO and GO after adjustment for age and sex (table 7.2, model 1). Fasting plasma 3-DG levels in individuals with IGM were 0.255 standard deviations higher than in those with NGM ( $\beta=$ $0.255 ; 95 \% \mathrm{Cl}: 0.136-0.375 ; \mathrm{p} \varangle 0.001$ ). Additional adjustment for smoking, eGFR, triglycerides, total-to-HDL cholesterol ratio, waist circumference, medication, prior CVD and systolic blood pressure did not materially change this association (table 7.2, model 2). Addition of fasting glucose levels to the regression model completely attenuated the association between the presence of IGM and higher fasting levels of 3-DG (table 7.2, model 3).

In contrast, the presence of type 2 diabetes was associated with higher fasting levels of both M GO ( $\beta=0.542 ; 95 \% \mathrm{Cl}$ : 0.322-0.761; $p \varangle 0.001)$ and 3-DG ( $\beta=1.517 ; 95 \% \mathrm{Cl}$ : 1.385 1.649; $p<0.001$ ) after adjustment for age and sex, as compared to NGM . After additional adjustment for multiple covariates in model 2 , associations between the presence of type 2 diabetes and higher fasting levels of M GO ( $\beta=0.492 ; 95 \% \mathrm{Cl}: 0.216-0.769 ; p \varangle 0.001)$ and 3-DG ( $\beta=1.376 ; 95 \% \mathrm{Cl}: 1.211-1.542 ; \quad<<0.001$ ) were largely unchanged. Further adjustment for fasting glucose levels resulted in an $82 \%$ attenuation of the association between type 2 diabetes and fasting MGO levels (table 7.2, model 3). In line, the association between type 2 diabetes and fasting 3-DG levels was attenuated by $61 \%$, but remained statistically significant (table 7.2, model 3). Also after adjustment for potential confounders, we found no associations between either IGM or type 2 diabetes and fasting $\mathrm{GO}$ levels.

\section{Multivariate associations between GM S and iAUCs of a-dicarbonyls}

In age- and sex-adjusted analyses, the presence of IGM was associated with significantly higher iAUCs for all three $\alpha$-dicarbonyls (MGO: $\beta=0.203 ; 95 \% \mathrm{Cl}$ : $0.123-0.283 ; p<0.001$, GO: $\beta=0.268 ; 95 \% \mathrm{Cl}: 0.160-0.377 ; p \varangle 0.001$ and 3-DG: $\beta=0.319 ; 95 \% \mathrm{Cl}: 0.257-0.382$; $\mathrm{p} \varangle 0.001$ ), as compared to NGM . For type 2 diabetes, the associations with higher iAUCs of all three $\alpha$-dicarbonyls appeared to be even stronger (MGO: $\beta=0.359 ; 95 \% \mathrm{Cl}$ : 0.271 0.448; $p \varangle 0.001, G 0: \beta=0.486 ; 95 \% \mathrm{Cl}: 0.366-0.606 ; p \varangle 0.001$ and 3-DG: $\beta=0.645 ; 95 \% \mathrm{Cl}$ : $0.576-0.714 ; p<0.001)$. After further adjustment for the covariates in model 2 , both IGM and type 2 diabetes remained significantly associated with higher iAUCs of all three $\alpha$-dicarbonyls (table 7.2, model 2), compared to iAUCs of individuals with NGM. However, when analyses were further adjusted for the iAUC from glucose, the associations of both IGM and type 2 diabetes with MGO and 3-DG iAUCs disappeared completely (table 7.2, model 3). 
Table 7.2. Associations between glucose metabolism status and fasting and iAUC measures of plasma a-dicarbonyls during an OGT

\begin{tabular}{|c|c|c|c|c|c|c|c|}
\hline \multirow{2}{*}{ Determinant } & \multirow{2}{*}{ Model } & \multicolumn{3}{|c|}{ IGM (vs NGM) } & \multicolumn{3}{|c|}{ Type 2 diabetes (vs NGM) } \\
\hline & & $\beta$ & $95 \% \mathrm{Cl}$ & $\mathrm{p}$-value & $\beta$ & $95 \% \mathrm{Cl}$ & $p$-value \\
\hline \multirow{3}{*}{ Fasting M GO } & 1 & 0.011 & $-0.188-0.209$ & 0.917 & 0.542 & $0.322-0.761$ & $<0.001$ \\
\hline & 2 & -0.019 & $-0.226-0.188$ & 0.855 & 0.492 & $0.216-0.769$ & $\varangle 0.001$ \\
\hline & 3 & -0.129 & $-0.343-0.085$ & 0.236 & 0.089 & $-0.268-0.447$ & 0.624 \\
\hline \multirow{3}{*}{ Fasting GO } & 1 & 0.030 & $-0.142-0.202$ & 0.735 & -0.112 & $-0.303-0.078$ & 0.247 \\
\hline & 2 & 0.063 & $-0.114-0.240$ & 0.486 & -0.088 & $-0.326-0.149$ & 0.465 \\
\hline & 3 & 0.013 & $-0.172-0.198$ & 0.891 & -0.272 & $-0.581-0.037$ & 0.085 \\
\hline \multirow{3}{*}{ Fasting 3-DG } & 1 & 0.255 & $0.136-0.375$ & $\varangle 0.001$ & 1.517 & $1.385-1.649$ & $\varangle 0.001$ \\
\hline & 2 & 0.216 & $0.092-0.341$ & $\varangle 0.001$ & 1.376 & $1.211-1.542$ & $\varangle 0.001$ \\
\hline & 3 & -0.013 & $-0.122-0.096$ & 0.815 & 0.536 & $0.354-0.718$ & $<0.001$ \\
\hline \multirow{4}{*}{ iAUC MGO } & 1 & 0.203 & $0.123-0.283$ & $\varangle 0.001$ & 0.359 & $0.271-0.448$ & $\varangle 0.001$ \\
\hline & 2 & 0.190 & $0.106-0.274$ & $\varangle 0.001$ & 0.293 & $0.180-0.405$ & $<0.001$ \\
\hline & 3 & 0.044 & $-0.043-0.130$ & 0.321 & -0.008 & $-0.137-0.120$ & 0.897 \\
\hline & 4 & 0.187 & $0.108-0.266$ & $\varangle 0.001$ & 0.362 & $0.255-0.469$ & $\varangle 0.001$ \\
\hline \multirow{4}{*}{ iAUC GO } & 1 & 0.268 & $0.160-0.377$ & $\varangle 0.001$ & 0.486 & $0.366-0.606$ & $\varangle 0.001$ \\
\hline & 2 & 0.287 & $0.172-0.401$ & $\varangle 0.001$ & 0.536 & $0.382-0.689$ & $<0.001$ \\
\hline & 3 & 0.114 & $-0.006-0.234$ & 0.064 & 0.180 & $0.001-0.358$ & 0.049 \\
\hline & 4 & 0.302 & $0.196-0.408$ & $\varangle 0.001$ & 0.514 & $0.372-0.656$ & $\varangle 0.001$ \\
\hline \multirow{4}{*}{ IAUC 3-DG } & 1 & 0.319 & $0.257-0.382$ & $\varangle 0.001$ & 0.645 & $0.576-0.714$ & $<0.001$ \\
\hline & 2 & 0.285 & $0.221-0.349$ & $\varangle 0.001$ & 0.542 & $0.456-0.628$ & $\varangle 0.001$ \\
\hline & 3 & 0.030 & $-0.013-0.072$ & 0.176 & 0.015 & $-0.048-0.079$ & 0.640 \\
\hline & 4 & 0.295 & $0.231-0.360$ & $\varangle 0.001$ & 0.607 & $0.500-0.714$ & $\varangle 0.001$ \\
\hline
\end{tabular}

$\overline{\text { Data were analyzed using linear regression analysis. The standardized regression coefficient } \beta \text { represents the }}$ increase of $\alpha$-dicarbonyl concentrations expressed in standard deviations, compared to NGM. MGO, methylglyoxal; GO, glyoxal; 3-DG, 3-deoxyglucosone; NGM, normal glucose metabolism; IGM, impaired glucose metabolism; $\mathrm{Cl}$, confidence interval; iAUC, incremental area under the curve.

Model 1: adjusted for age +sex;

Model 2: model 1 +smoking + eGFR + triglycerides + total-to-HDL cholesterol ratio + waist circumference + prior CVD + systolic blood pressure + medication (lipid-lowering, glucose-lowering and blood pressure-lowering medication);

Model 3: model 2 +glucose (fasting glucose for fasting $\alpha$-dicarbonyls and iAUC glucose for iAUC $\alpha$-dicarbonyls); Model 4: model 2 +fasting levels of corresponding $\alpha$-dicarbonyls. 
Similarly, although the association between type 2 diabetes and higher iAUC of GO $(\beta=0.180 ; 95 \% \mathrm{Cl}: 0.001-0.358 ; p=0.049$ ) remained significant, it was also attenuated by $66 \%$. Adjustment for fasting levels of the appropriate $\alpha$-dicarbonyls in model 4 did not change the strength of the association compared to model 2 (table 7.3, model 4 vs. 2).

Since the associations between IGM and type 2 diabetes and higher $\alpha$-dicarbonyl iAUCS almost completely disappeared after adjustment for glucose, we hypothesized that the higher iAUCs of MGO, GO and 3-DG were a direct result of higher iAUCs of glucose in IGM and type 2 diabetes, rather than a decreased capacity to detoxify $\alpha$-dicarbonyls.

A

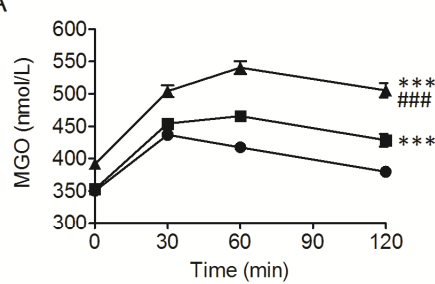

C

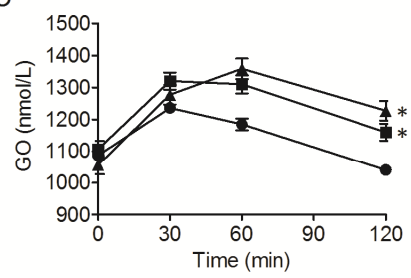

E

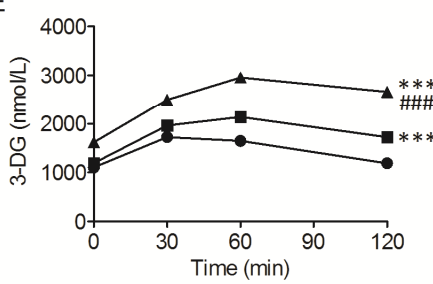

G

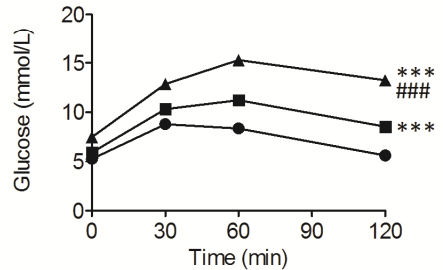

B

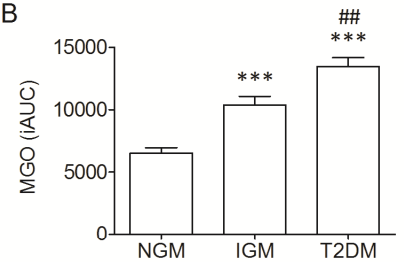

D

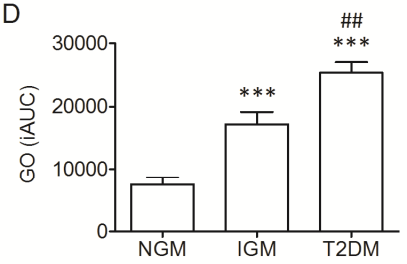

$\mathrm{F}$

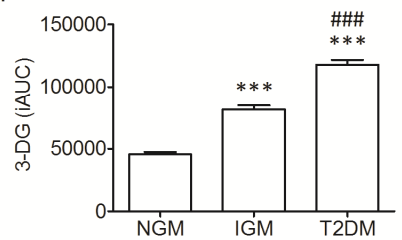

$\mathrm{H}$

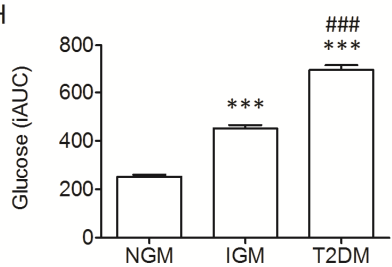

Figure 7.2. Plasma $\alpha$-dicarbonyl and glucose levels during an oral glucose tolerance test. (A) shows M GO levels during an OGTT over time and (B) demonstrates these data calculated as an iAUC. (C-D) shows the same for GO levels, (E-F) for 3-DG levels and (G-H) for glucose levels. Data are shown as means \pm standard error of the means. Circles $=$ NGM , squares $=$ IGM and triangles $=$ type 2 diabetes. Differences in OGT curves between the groups of GMS were tested with repeated measures two-way ANOVA with Bonferroni correction. Differences in iAUCs between the groups were tested with one-way ANOVA with Bonferroni correction. $* * * p<0.001, * p<0.05$ compared to NGM and \#\# $\measuredangle 0.001$, \#p $\measuredangle 0.01$ compared to IGM. MGO, methylglyoxal; G0, glyoxal; 3-DG, 3deoxyglucosone; OGT, oral glucose tolerance test; iAUC, incremental area under the curve; NGM, normal glucose metabolism; IGM, impaired glucose metabolism. 
Indeed, when adjusted for all covariates in model 2, the glucose iAUC was strongly associated with the iAUC of all three $\alpha$-dicarbonyls (table 7.3 ; MGO: $\beta=0.195 ; 95 \% \mathrm{Cl}$ : $0.148-0.243 ; p \varangle 0.001, \mathrm{GO}: \beta=0.231 ; 95 \% \mathrm{Cl}: 0.165-0.297 ; p \varangle 0.001$ and 3-DG: $\beta=0.342$; $95 \% \mathrm{Cl}: 0.318-0.365 ; p \varangle 0.001)$. These associations did not differ between NGM, IGM or type 2 diabetes for all three $\alpha$-dicarbonyls (pinteraction $>0.1$ ). Overall, associations between the IAUC of glucose and the iAUC of $\alpha$-dicarbonyls were stronger than associations between $\mathrm{HbAlC}$ or fasting plasma glucose and the IAUC of $\alpha$-dicarbonyls (table 7.3).

Table 7.3. Associations of iAUC glucose, HbAlC and fasting glucose with iAUC of plasma $\alpha$-dicarbonyls

\begin{tabular}{|c|c|c|c|c|c|c|c|c|c|c|}
\hline \multirow{2}{*}{ Determinant } & \multirow{2}{*}{ Model } & \multicolumn{3}{|c|}{ iAUC Glucose } & \multicolumn{3}{|c|}{$\mathrm{HbA}_{1 \mathrm{c}}$} & \multicolumn{3}{|c|}{ Fasting glucose } \\
\hline & & $\beta$ & $95 \% \mathrm{Cl}$ & p-value & $\beta$ & $95 \% \mathrm{Cl}$ & p-value & $\beta$ & $95 \% \mathrm{Cl}$ & p-value \\
\hline \multirow{4}{*}{ ¡AUC MGO } & 1 & 0.188 & $0.143-$ & $\varangle 0.001$ & 0.015 & $-0.026-$ & 0.464 & -0.002 & -0.069 & 0.942 \\
\hline & & & 0.233 & & & 0.057 & & & 0.064 & \\
\hline & 2 & 0.195 & $0.148-$ & $\varangle 0.001$ & 0.004 & -0.039 & 0.865 & -0.023 & $-0.092-$ & 0.521 \\
\hline & & & 0.243 & & & 0.046 & & & 0.046 & \\
\hline \multirow{4}{*}{ ¡AUC GO } & 1 & 0.201 & $0.138-$ & $\varangle 0.001$ & 0.020 & $-0.035-$ & 0.470 & -0.059 & -0.149 & 0.194 \\
\hline & & & 0.264 & & & 0.075 & & & 0.030 & \\
\hline & 2 & 0.231 & $0.165-$ & $\varangle 0.001$ & 0.019 & -0.039 & 0.522 & -0.058 & $-0.152-$ & 0.228 \\
\hline & & & 0.297 & & & 0.076 & & & 0.036 & \\
\hline \multirow{4}{*}{ IAUC 3-DG } & 1 & 0.337 & $0.314-$ & $\varangle 0.001$ & 0.064 & $0.028-$ & 0.001 & 0.032 & -0.019 & 0.222 \\
\hline & & & 0.360 & & & 0.099 & & & 0.084 & \\
\hline & 2 & 0.342 & $0.318-$ & $\varangle 0.001$ & 0.048 & $0.012-$ & 0.011 & 0.005 & $-0.048-$ & 0.857 \\
\hline & & & 0.365 & & & 0.085 & & & 0.058 & \\
\hline
\end{tabular}

Data were analyzed using linear regression analysis. The standardized regression coefficient $\beta$ represents the increase of $\alpha$-dicarbonyl concentrations expressed in standard deviations per standard deviation increase in iAUC glucose, HbA1C and fasting glucose. MGO, methylglyoxal; GO, glyoxal; 3-DG, 3-deoxyglucosone; Cl, confidence interval; iAUC, incremental area under the curve.

Model 1: adjusted for age + sex + glucose metabolism status;

Model 2: model 1 +smoking + eGFR + triglycerides + total-to-HDL cholesterol ratio + waist circumference + prior CVD + systolic blood pressure + medication (lipid-lowering, glucose-lowering and blood pressure-lowering medication).

\section{Conclusions}

This study demonstrated that iAUCS of the $\alpha$-dicarbonyls MGO, GO and 3-DG, as calculated from an OGT, were higher in individuals with IGM and type 2 diabetes, independently of potential confounders. Fasting plasma levels of $\alpha$-dicarbonyls were predominantly higher in individuals with type 2 diabetes, although fasting 3-DG levels were also slightly increased in individuals with IGM. After adjustment for glucose, these associations disappeared almost completely for MGO and 3-DG, and to a large, but slightly lesser extent, for $\mathrm{GO}$, indicating that glucose is the major source of $\alpha$-dicarbonyls. To our knowledge, this is the first time that $\alpha$-dicarbonyls have been measured in a postglucose load setting in individuals with NGM , IGM and type 2 diabetes. 
In the present study, we found that iAUCs of MGO, GO and 3-DG, as calculated from an OGT, were higher in individuals with IGM and type 2 diabetes, independently of potential confounders. Our data are in accordance with findings from a previous study by Beisswenger et al, in which they showed increased MGO and 3-DG levels during the postprandial period in patients with type 1 diabetes $^{20}$. As glucose serves as a primary source for the formation of $\alpha$-dicarbonyls, transient glucose excursions during the postprandial period may give rise to increases of $\alpha$-dicarbonyl levels, which in turn may induce long-term damage to the vasculature. Indeed, El-Osta et al. demonstrated that even transient exposures to high glucose levels induce persistent changes in cultured endothelial cells, which could be prevented by an overexpression of glyoxalase I (GLO1), the major enzyme detoxifying $\mathrm{MGO}$ and $\mathrm{GO}^{28}$. We and others demonstrated that increased levels of $\alpha$-dicarbonyl compounds are directly associated with vascular complications $^{14,29}$. MGO particularly has attracted a lot of attention as a key player in vascular dysfunction, due to its capacity to induce oxidative stress ${ }^{29}$, cell death ${ }^{30}$ and endothelial dysfunction ${ }^{14}$. Therefore, the observed increased levels of $\alpha$-dicarbonyls with post-challenge glucose excursions in IGM and type 2 diabetes may link fluctuations in blood glucose levels in these patients with persistent increases in risk of vascular complications and CVD.

The $\alpha$-dicarbonyls in the plasma can originate from various sources, including in situ formation in the plasma, release from cells and external sources ${ }^{31}$. Due to their rapid increase during the OGT, the post-load plasma $\alpha$-dicarbonyl levels most likely originate from intracellular compartments which come directly into contact with plasma glucose. As we found the largest increase in $\alpha$-dicarbonyls in the individuals with IGM and with type 2 diabetes, it is likely that plasma $\alpha$-dicarbonyls originate from insulin-independent cells, specifically red blood cells and endothelial cells. Indeed, we demonstrated in human endothelial cells that hyperglycemia produced higher levels of $\mathrm{MGO}^{32}$ and that $\alpha$-dicarbonyl levels are much higher in circulating cells than in plasma ${ }^{23}$. We assume that $\alpha$-dicarbonyl levels after a glucose challenge would increase even further in circulating cells and endothelial cells, as only a small percentage of dicarbonyl compounds leaks into the circulation ${ }^{23}$. To what extent this increase of dicarbonyls in plasma is similar in other tissues that are sensitive to diabetic complications, such as the kidney, nerves, retina and atherosclerotic plaques, remains to be elucidated. Next to release from cells, plasma $\alpha-$ dicarbonyls may also be a result of autoxidation of glucose in the plasma, but it is not known to which extent this process contributes to plasma levels of $\alpha$-dicarbonyls. It is unlikely however that the iAUCs reflect any autoxidation, as this is a slow process and we observed an increase of $\alpha$-dicarbonyls already ${ }^{30}$ minutes after the glucose load. In addition, dicarbonyl compounds can also originate from exogenous sources ${ }^{31}$, for example from the glucose drink we used in this study. However, we previously observed no increased plasma MGO levels in a healthy volunteer within two hours after drinking coffee, a drink with very high levels of MGO $(55 \mu \mathrm{mol} / \mathrm{L})$ but without any glucose. As the glucose drink used for the OGTT only contained $1.6 \mu \mathrm{mol} / \mathrm{L} \mathrm{MGO}$, it is highly unlikely that the low levels of $\alpha$-dicarbonyls in the glucose drink contribute to their plasma levels after the glucose challenge. 
The question remains whether the increased $\alpha$-dicarbonyl iAUCs are a reflection of decreased detoxification potency in IGM and type 2 diabetes. The glyoxalase system is the major pathway to detoxify $M G O$ and $\mathrm{GO}^{33}$. This system consists of the rate-limiting enzyme glyoxalase-1 and glyoxalase-2. These enzymes convert MGO and GO, with the involvement of reduced glutathione, to their end product D-lactate ${ }^{33}$. Several experimental studies have linked the presence of diabetes to dysfunction of the glyoxalase system ${ }^{34}$. However, additional adjustment for glucose in our analyses attenuated the associations between GMS and the iAUC of $\alpha$-dicarbonyls by $60-97 \%$, suggesting that the elevated post-challenge dicarbonyl levels are the result of increased formation from its substrate glucose. Although dysfunction of $\alpha$-dicarbonyl detoxification cannot be eliminated as a contributor to increased plasma $\alpha$-dicarbonyl levels, the lack of interaction between dicarbonyl iAUCs and GMS indicates that detoxification mechanisms are not differently active in any of the three GM S groups.

Our new observation that post-load glucose levels are closely associated with $\alpha$-dicarbonyl formation is of high clinical relevance because of the current developments in the field of glucose-lowering therapies. Dipeptidyl-peptidase-4 (DPP-4) inhibitors form a very new treatment strategy which has been shown to regulate postprandial glucose concentrations ${ }^{35}$. Whether DPP-4 inhibitors can reduce $\alpha$-dicarbonyls is unknown. In addition, the bionic pancreas is a state of the art intervention which has been demonstrated to regulate glycemic control very strictly in type 1 diabetes ${ }^{36}$, and may prove valuable in type 2 diabetes as well. Directly lowering $\alpha$-dicarbonyl levels may also be a mechanism to reduce postprandial carbonyl stress and the putative association with vascular damage at long term. One intervention which is currently highly under investigation is pyridoxamine. Pyridoxamine is a chemical scavenger of reactive $\alpha$-dicarbonyls and has been shown to inhibit formation of AGEs. Several experimental and clinical studies have already demonstrated beneficial effects of pyridoxamine with regard to diabetic microvascular complications $\mathrm{s}^{37-39}$.

The major strengths of this study are that we were able to perform our analyses in a large and well-defined cohort study, and, in addition, we measured plasma $\alpha$-dicarbonyls with state of the art techniques based on UPLC-MS/MS. There are also a few limitations of this study. First, individuals with known type 2 diabetes did not undergo an OGT, while they had higher fasting levels of $\alpha$-dicarbonyl compounds compared to the type 2 diabetes patients who did undergo a full OGTT. Therefore, their $\alpha$-dicarbonyl levels during the OGTT are expected to increase even more than in newly diagnosed subjects, indicating that our observations may be an underestimation of the true effect in type 2 diabetes. Furthermore, the glucose load in the OGTT is not completely comparable to postprandial glucose excursions. To investigate the effect of postprandial glucose excursions on $\alpha$ dicarbonyl levels, a mixed meal test needs to be done, although it has been described that the level of glycemia $2 \mathrm{~h}$ after an OGTT is closely related to the level of glycemia after a standardized meal ${ }^{40}$.

Moreover, a major advantage of the OGTT used in this study is that it allowed us to specifically investigate the hypothesis that glucose spikes cause formation of MGO, without confounding of postprandial changes in lipid and protein levels. 
In conclusion, we found that significant increases of MGO, GO and 3-DG levels occurred during an OGTT in individuals with IGM and type 2 diabetes in comparison to controls. These increases were strongly associated with post-load glucose excursions. These findings, together with the known harmful biological effects of these $\alpha$-dicarbonyls, underline the potential importance of dicarbonyl stress as a functional candidate to explain the increased risk of diabetic complications in individuals with postprandial hyperglycemia. Prospective analyses on micro- and macrovascular complications are necessary to associate our current findings with vascular outcome. 


\section{References}

1

2

3

4

5

6

Forbes, J. M. \& Cooper, M. E. Mechanisms of diabetic complications. Physiological reviews 93, 137188, doi:10.1152/physrev.00045.2011 (2013).

$$
\text { de Vegt, F. et al. Hyperglycaemia is associated with all-cause and cardiovascular mortality in the }
$$
Hoorn population: the Hoorn Study. Diabetologia 42, 926-931, doi:10.1007/s001250051249 (1999).

3 Beisswenger, P., Heine, R. J., Leiter, L. A., Moses, A. \& Tuomilehto, J. Prandial glucose regulation in the glucose triad: emerging evidence and insights. Endocrine 25, 195-202, doi:10.1385/ENDO:25:3:195 (2004).

The effect of intensive treatment of diabetes on the development and progression of long-term complications in insulin-dependent diabetes mellitus. The Diabetes Control and Complications Trial Research Group. The New England journal of medicine 329, 977-986, doi:10.1056/NEJM 199309303291401 (1993).

Intensive blood-glucose control with sulphonylureas or insulin compared with conventional treatment and risk of complications in patients with type 2 diabetes (UKPDS 33). UK Prospective Diabetes Study (UKPDS) Group. Lancet 352, 837-853 (1998).

6 Rohlfing, C. L. et al. Defining the relationship between plasma glucose and $\mathrm{HbA}(1 \mathrm{C})$ : analysis of glucose profiles and $\mathrm{HbA}(\mathrm{lc})$ in the Diabetes Control and Complications Trial. Diabetes care 25, 275278 (2002).

Donahue, R. P., Abbott, R. D., Reed, D. M. \& Yano, K. Postchallenge glucose concentration and coronary heart disease in men of Japanese ancestry. Honolulu Heart Program. Diabetes 36, 689-692 (1987).

8 Lowe, L. P. et al. Diabetes, asymptomatic hyperglycemia, and 22-year mortality in black and white men. The Chicago Heart Association Detection Project in Industry Study. Diabetes care 20, 163-169 (1997).

Glucose tolerance and mortality: comparison of WHO and American Diabetes Association diagnostic criteria. The DECODE study group. European Diabetes Epidemiology Group. Diabetes Epidemiology: Collaborative analysis Of Diagnostic criteria in Europe. Lancet 354, 617-621 (1999).

0 Coutinho, M., Gerstein, H. C., Wang, Y. \& Yusuf, S. The relationship between glucose and incident cardiovascular events. A metaregression analysis of published data from 20 studies of 95,783 individuals followed for 12.4 years. Diabetes care 22, 233-240 (1999).

1 Balkau, B. et al. High blood glucose concentration is a risk factor for mortality in middle-aged nondiabetic men. 20-year follow-up in the Whitehall Study, the Paris Prospective Study, and the Helsinki Policemen Study. Diabetes care 21, 360-367 (1998).

Hanefeld, M. et al. Risk factors for myocardial infarction and death in newly detected NIDDM : the Diabetes Intervention Study, 11-year follow-up. Diabetologia 39, 1577-1583 (1996).

Cavalot, F. et al. Postprandial blood glucose is a stronger predictor of cardiovascular events than fasting blood glucose in type 2 diabetes mellitus, particularly in women: lessons from the San Luigi Gonzaga Diabetes Study. The Journal of clinical endocrinology and metabolism 91, 813-819, doi:10.1210/jc.2005-1005 (2006). 
Brouwers, 0. et al. Glyoxalase-1 overexpression reduces endothelial dysfunction and attenuates early renal impairment in a rat model of diabetes. Diabetologia 57, 224-235, doi:10.1007/500125-0133088-5 (2014).

Giacco, F. et al. Knockdown of glyoxalase 1 mimics diabetic nephropathy in nondiabetic mice. Diabetes 63, 291-299, doi:10.2337/db13-0316 (2014).

Berner, A. K. et al. Protection against methylglyoxal-derived AGEs by regulation of glyoxalase 1 prevents retinal neuroglial and vasodegenerative pathology. Diabetologia 55, 845-854, doi:10.1007/s00125-011-2393-0 (2012).

17 Duran-Jimenez, B. et al. Advanced glycation end products in extracellular matrix proteins contribute to the failure of sensory nerve regeneration in diabetes. Diabetes 58, 2893-2903, doi:10.2337/db090320 (2009).

Bierhaus, A. et al. Methylglyoxal modification of Nav1.8 facilitates nociceptive neuron firing and causes hyperalgesia in diabetic neuropathy. Nature medicine 18, 926-933, doi:10.1038/nm.2750 (2012).

Tikellis, C. et al. Dicarbonyl stress in the absence of hyperglycemia increases endothelial inflammation and atherogenesis similar to that observed in diabetes. Diabetes, doi:10.2337/db13-0932 (2014). Beisswenger, P. J. et al. alpha-Dicarbonyls increase in the postprandial period and reflect the degree of hyperglycemia. Diabetes care 24, 726-732 (2001).

$\mathrm{Du}, \mathrm{H}$. et al. Glycemic index and glycemic load in relation to food and nutrient intake and metabolic risk factors in a Dutch population. The American journal of clinical nutrition 87, 655-661 (2008).

Hanssen, N. M. et al. Plasma levels of advanced glycation endproducts Nepsilon(carboxymethyl)lysine, Nepsilon-(carboxyethyl)/ysine, and pentosidine are not independently associated with cardiovascular disease in individuals with or without type 2 diabetes: the Hoorn and CODAM studies. The Journal of clinical endocrinology and metabolism 98, E1369-1373, doi:10.1210/jc.2013-1068 (2013).

23 Scheijen, J. L. \& Schalkwijk, C. G. Quantification of glyoxal, methylglyoxal and 3-deoxyglucosone in blood and plasma by ultra performance liquid chromatography tandem mass spectrometry: evaluation of blood specimen. Clinical chemistry and laboratory medicine : CCLM / FESCC 52, 85-91, doi:10.1515/cclm-2012-0878 (2014).

24 Allison, D. B., Paultre, F., Maggio, C., M ezzitis, N. \& Pi-Sunyer, F. X. The use of areas under curves in diabetes research. Diabetes care 18, 245-250 (1995).

25 van Greevenbroek, M. M. et al. The cross-sectional association between insulin resistance and circulating complement $\mathrm{C} 3$ is partly explained by plasma alanine aminotransferase, independent of central obesity and general inflammation (the CODAM study). European journal of clinical investigation 41, 372-379, doi:10.1111/j.1365-2362.2010.02418.x (2011). Levey, A. S. et al. A new equation to estimate glomerular filtration rate. Annals of internal medicine 150, 604-612 (2009). Jacobs, M. et al. Low-grade inflammation can partly explain the association between the metabolic syndrome and either coronary artery disease or severity of peripheral arterial disease: the CODAM study. European journal of clinical investigation 39, 437-444, doi:10.1111/j.1365-2362.2009.02129.x (2009). 
El-Osta, A. et al. Transient high glucose causes persistent epigenetic changes and altered gene expression during subsequent normoglycemia. The Journal of experimental medicine 205, 2409-2417, doi:10.1084/jem.20081188 (2008).

29 Brouwers, 0 . et al. Overexpression of glyoxalase-I reduces hyperglycemia-induced levels of advanced glycation end products and oxidative stress in diabetic rats. The Journal of biological chemistry $\mathbf{2 8 6}$, 1374-1380, doi:10.1074/jbc.M 110.144097 (2011).

30 Kang, Y., Edwards, L. G. \& Thornalley, P. J. Effect of methylglyoxal on human leukaemia 60 cell growth: modification of DNA G1 growth arrest and induction of apoptosis. Leukemia research $\mathbf{2 0}$, 397-405 (1996).

Kalapos, M. P. Where does plasma methylglyoxal originate from? Diabetes research and clinical practice 99, 260-271, doi:10.1016/j.diabres.2012.11.003 (2013).

Schalkwijk, C. G. et al. Heat-shock protein 27 is a major methylglyoxal-modified protein in endothelial cells. FEBS letters 580, 1565-1570, doi:10.1016/j.febslet.2006.01.086 (2006).

33 Thornalley, P. J. The glyoxalase system in health and disease. Molecular aspects of medicine 14, 287371 (1993).

4 Rabbani, N. \& Thornalley, P. J. Glyoxalase in diabetes, obesity and related disorders. Seminars in cell \& developmental biology 22, 309-317, doi:10.1016/j.semcdb.2011.02.015 (2011).

Vora, J. et al. Incretin-based therapy in combination with basal insulin: a promising tactic for the treatment of type 2 diabetes. Diabetes \& metabolism 39, 6-15, doi:10.1016/j.diabet.2012.08.002 (2013).

Russell, S. J. et al. Outpatient glycemic control with a bionic pancreas in type 1 diabetes. The New England journal of medicine 371, 313-325, doi:10.1056/NEJM oa1314474 (2014).

Williams, M. E. et al. Effects of pyridoxamine in combined phase 2 studies of patients with type 1 and type 2 diabetes and overt nephropathy. Am J Nephrol 27, 605-614, doi:10.1159/000108104 (2007).

8 Tanimoto, M. et al. Effect of pyridoxamine (K-163), an inhibitor of advanced glycation end products, on type 2 diabetic nephropathy in KK-A(y)/Ta mice. Metabolism: clinical and experimental 56, 160 167, doi:10.1016/j.metabol.2006.08.026 (2007).

9 Degenhardt, T. P. et al. Pyridoxamine inhibits early renal disease and dyslipidemia in the streptozotocin-diabetic rat. Kidney international 61, 939-950, doi:10.1046/j.1523-1755.2002.00207.x (2002).

Wolever, T. M. et al. Variation of postprandial plasma glucose, palatability, and symptoms associated with a standardized mixed test meal versus $75 \mathrm{~g}$ oral glucose. Diabetes care 21, 336-340 (1998). 



\section{ANALY SIS OF ADVANCED CLY CATION}

ENDPRODUCTS IN SELECTED

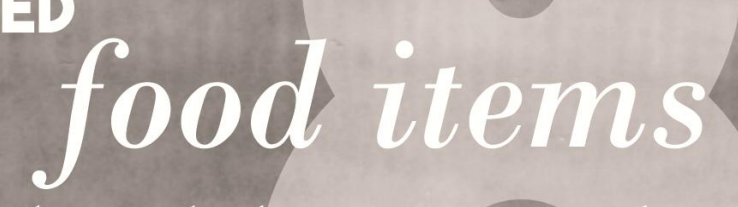

by ultra-performance liquid chromatography tandem mass spectrometry; PRESENTAIION OF A DIETARY ACE DATABASE 


\section{8}

Analysis of advanced glycation endproducts in selected food items by ultra-performance liquid chromatography tandem mass spectrometry; presentation of a dietary AGE database

Jean L.J.M. Scheijen, Egbert Clevers, Lian Engelen, Pieter C. Dagnelie, Fred Brouns, Coen D.A. Stehouwer and Casper G. Schalkwijk 


\section{Abstract}

The aim of this study was to validate an ultra-performance liquid chromatography tandem mass-spectrometry (UPLC-MS/MS) method for the determination of advanced glycation endproducts (AGEs) in food items and to analyze AGEs in a selection of food items commonly consumed in a Western diet. $N^{\varepsilon}$-(carboxymethyl)lysine (CML), $\mathrm{N}^{\varepsilon}$-(1-carboxyethyl)lysine (CEL) and $\mathrm{N}^{\delta}$-(5-hydro-5-methyl-4-imidazolon-2-yl)-ornithine (M G-H1) were quantified in the protein fractions of 190 food items using UPLC-M S/MS. Intra- and inter-day accuracy and precision were 2-29\%. The calibration curves showed perfect linearity in water and food matrices. We found the highest AGE levels in high-heat processed nut or grain products, and canned meats. Fruits, vegetables, butter and coffee had the lowest AGE content. The described method proved to be suitable for the quantification of three major AGEs in food items. The presented dietary AGE database opens the possibility to further quantify actual dietary exposure to AGEs and to explore its physiological impact on human health. 


\section{Introduction}

The non-enzymatic reaction of sugars with proteins, better known as the Maillard or browning reaction, leads to formation of advanced glycation endproducts (AGEs) ${ }^{1,2}$. This reaction was described for the first time in 1912 by the food chemist Louis Camille Maillard ${ }^{3}$. It was not until 1980 that the pathophysiological significance of AGEs emerged in medical science ${ }^{1,2}$. The accumulation of AGEs in the body has been implicated in the pathogenesis of age-related diseases in particular in relation to diabetes, inflammation, neurodegenerative disorders and cardiovascular disease ${ }^{4-7}$. Several mechanisms have been proposed by which AGEs may adversely affect human health, including the binding of AGEs to AGE-receptors, and aberrant cross-linking in the extracellular matrix ${ }^{8}$.

The group of AGEs is a heterogeneous family of unavoidable by-products which are endogenously formed by reactive metabolic intermediates derived from sugars and from lipid peroxidation. AGEs formed by sugar-derived carbonyls ${ }^{9}$ include $\mathrm{N}^{\varepsilon}$-(1-carboxyethyl)|ysine (CEL) and $\mathrm{N}^{\delta}$-(5-hydro-5-methyl-4-imidazolon-2-yl)-ornithine (M G-H1); AGEs formed by lipid peroxidation and oxidative stress ${ }^{10,11}$ include in particular $\mathrm{N}^{\varepsilon}$-(carboxymethyl)lysine (CM L), a well-known ligand for the receptor for AGES (RAGE) ${ }^{12}$.

The processing and preparation of foods is also susceptible to AGE formation with high temperatures as a potent promoter ${ }^{13-16}$. Animal studies have shown that some of these dietary AGEs are absorbed and that these AGEs, similar to their endogenous counterparts, manifest pathological effects, such as inducing insulin resistance ${ }^{16-18}$. In humans, high- vs. low-CML diets have been shown to increase levels of serum AGEs and serum markers of inflammation and endothelial dysfunction, and to impair flow-mediated dilation and insulin sensitivity ${ }^{19-23}$. However, reliable quantification of AGEs in food, has thus far been a matter of debate ${ }^{24}$.

To substantiate whether dietary AGEs pose a risk to human health, a first crucial step is to have access to an accurate method to quantify AGEs and to establish a reliable dietary AGE database. Aside from several reports of small studies, four major studies reported on the AGE content in $\sim 250$ to 1500 selected foods and beverages ${ }^{25-28}$. Three of these studies described only the $C M L$ content ${ }^{25-27}$. M oreover, three of these studies were based on the determination of AGEs by ELISA ${ }^{25,26,28}$, but AGE measurements with immunoassays yield only semi-quantitative results and should thus be interpreted with caution ${ }^{16,24}$. A much better approach for the quantitative determination of specific AGE epitopes in proteins is the use of specific analytical techniques ${ }^{29}$. Several smaller studies have described the detection and quantification of AGEs in food with gas chromatography mass spectrometry (GC-MS S) ${ }^{15,24}$, high performance liquid chromatography with UV detection (HPLC-UV) $)^{15}$ or fluorescence detection ${ }^{30}$ and with liquid chromatography mass spectrometry $(L C-M S)^{13,29,31-33}$. However, only the quantification of one AGE $(C M L)^{24,31,33}$ and/or the AGE content of just one food product or food category was reported ${ }^{13,15,29,32,34}$. 
In the present study we describe a highly sensitive, specific and rapid ultra-performance liquid chromatography tandem mass spectrometry (UPLC-MS/MS) method for the analysis of $\mathrm{CML}, \mathrm{CEL}$ and MG-H1 in the protein fraction in food items. We achieved a significant improvement over the methods previously described by using stable isotope labeled internal standards and analysis of three important AGEs. With this validated method, we quantified the concentration of these AGEs in 190 food items which were chosen based upon their habitual and quantitative consumption in the Dutch population.

\section{Materials and Methods}

\section{Materials}

Boric acid, sodium borohydride, trifluoroacetic acid (TFA) and chloroform were obtained from Sigma-Aldrich (Zwijndrecht, the Netherlands). Butanol and methanol were obtained from Baker (Deventer, the Netherlands). Sodium hydroxide and hydrochloric acid were obtained from Merck (Darmstadt, Germany). Water and acetonitrile were obtained from Biosolve BV (Valkenswaard, the Netherlands). CM L (99\%), [ $\left.{ }^{2} \mathrm{H}_{2}\right]-\mathrm{CM} \mathrm{L}$ (95.7\%), CEL (98.6\%), $\left[{ }^{2} \mathrm{H}_{4}\right]$-CEL, M G-H1 (92.6\%) and $\left[{ }^{2} \mathrm{H}_{3}\right]-\mathrm{MG}-\mathrm{H} 1$ (98.7\%) were obtained from Polypeptide. All other reagents and solvents were of analytical or UPLC/M S grade.

\section{Selection of food items for AGE analysis}

Food items for AGE analysis were selected based on the items included in the Dutch cohort of the European Prospective Investigation into Cancer and Nutrition (EPIC) 35 and the Dutch National Food Consumption Survey 36. A total of 190 food items were chosen based upon their habitual and frequent consumption by the Dutch population.

\section{Sample preparation protein-bound AGEs}

Food items were obtained from local supermarkets and prepared according to the instructions on the manufacturer's label or obtained as prepared food from restaurants (supplemental table, table s8.1). To prevent neo-formation of CM L during acid hydrolysis a reduction step with sodium borohydride was used. To check the efficiency of this reduction step a peanut butter sample ( $\sim 5 \mathrm{mg})$, a Dutch spiced cake sample ( $17 \mathrm{mg})$ and a milk sample $(50 \mu \mathrm{L})$, was incubated with $200 \mu \mathrm{l}$ of $0,50,100,200$ and $400 \mathrm{mmol} / \mathrm{L}$ sodium borohydride dissolved in $200 \mathrm{mmol} / \mathrm{L}$ borate buffer $(\mathrm{pH} \mathrm{9.2)}$ for two hours at room temperature. Moreover, the efficiency of the incubation time was investigated by incubating these samples with $100 \mathrm{mmol} / \mathrm{L}$ sodium borohydride dissolved in $200 \mathrm{mmol} / \mathrm{L}$ borate buffer ( $\mathrm{pH}$ 9.2) for 0, 2, 4 and 6 hours. Food items containing $<20 \%$ fat were mixed and subsequently deproteinized with $1000 \mu \mathrm{l}$ cold $\left(4^{\circ} \mathrm{C}\right)$ TFA. 
Food items $>20 \%$ fat were mixed and subsequently deproteinized with a mixture of chloroform:methanol $(2: 1, \mathrm{v} / \mathrm{v}) 37$. After centrifugation $\left(4300 \mathrm{~g}, 4^{\circ} \mathrm{C}, 20 \mathrm{~min}\right)$ the supernatant (TFA) or lower phase (chloroform) was carefully removed with a Pasteur pipette. For validation experiments $25 \mu \mathrm{L}$ of a standard solution (six point calibration curve; 5250-0 nmol/L CM L, 6250-0 nmol/L CEL and 14749-0 nmol/L M G-H1) was added. Samples were then hydrolyzed by adding $500 \mu \mathrm{l} 6 \mathrm{~N} \mathrm{HCl}$ and incubated for 24 hours at $110^{\circ} \mathrm{C}$. After hydrolysis, $40 \mu \mathrm{L}$ hydrolysate and $20 \mu \mathrm{L}$ internal standard (containing $1432 \mathrm{nmol} / \mathrm{L}\left[{ }^{2} \mathrm{H}_{2}\right]-\mathrm{CML}, 1378 \mathrm{nmol} / \mathrm{L}\left[{ }^{2} \mathrm{H}_{4}\right]-\mathrm{CEL}$ and $\left.1322 \mathrm{nmol} / \mathrm{L}\left[{ }^{2} \mathrm{H}_{3}\right]-\mathrm{MG}-\mathrm{H} 1\right)$ were mixed in a reaction vial. This mixture was evaporated to dryness under a stream of nitrogen gas at $70^{\circ} \mathrm{C}$. To increase sensitivity during electrospray ionization and retention time using reversed phase chromatography, samples were subsequently derivatized with $100 \mu \mathrm{L}$ 1-butanol: $\mathrm{HCl}(3: 1, \mathrm{v} / \mathrm{v})$ for 90 minutes at $70^{\circ} \mathrm{C}$. After derivatisation the samples were evaporated to dryness under nitrogen, redissolved in $300 \mu \mathrm{L}$ water, mixed and subsequently centrifuged at $20000 \mathrm{~g}$ for 20 minutes.

\section{UPLC tandem MS analysis}

Derivatized $\mathrm{CML}, \mathrm{CEL}$ and $\mathrm{MG}-\mathrm{H} 1$ were analyzed by ultra-performance liquid chromatography (Acquity UPLC, Waters, Milford, USA) and detected in ESI positive multiple reaction monitoring (M RM) mode using a Xevo TQ MS (Waters, Milford, USA). Derivatives were separated on a reversed-phase $\mathrm{C} 18$ column (Acquity UPLC BEH C18, $50 \mathrm{x}$ $2.1 \mathrm{~mm}, 1.7 \mu \mathrm{m}$ ) with a linear gradient of $5 \mathrm{mmol} / \mathrm{L}$ ammonia and acetonitril at $48^{\circ} \mathrm{C}$. The flow rate was $800 \mu \mathrm{l} / \mathrm{min}$ and the injection volume was $2 \mu \mathrm{L}$. Optimal conditions for all parents and daughters were obtained by direct infusion of standard solutions of $\mathrm{CML}$ (5250 nmol/L), CEL (6250 nmol/L), MG-H1 (14749 nmol/L), $\left[{ }^{2} \mathrm{H}_{2}\right]-\mathrm{CML}(1432 \mathrm{nmol} / \mathrm{L})$, $\left[{ }^{2} \mathrm{H}_{4}\right]$-CEL (1378 nmol/L) and $\left.{ }^{2} \mathrm{H}_{3}\right]-\mathrm{M} \mathrm{G}-\mathrm{H} 1$ (1322 nmol/L). The M RM transitions for $\mathrm{CML}$, CEL and M G-H1 were respectively 317.1>186.1, 331.1>186.1 and 285.1>172.1. The M RM transitions for the internal standards $\left[{ }^{2} \mathrm{H}_{2}\right]-\mathrm{CML},\left[{ }^{2} \mathrm{H}_{4}\right]-\mathrm{CEL}$, and $\left[{ }^{2} \mathrm{H}_{3}\right]-\mathrm{MG}-\mathrm{H} 1$ were respectively $319.1>186.1,335.1>190.1$ and $288.1>172.1$. Electrospray ionization was found optimal at a capillary voltage of $0.5 \mathrm{kV}$ a source temperature of $150^{\circ} \mathrm{C}$ and a desolvation temperature of $600^{\circ} \mathrm{C}$. Quantification of CM L, CEL and MG-H1 was performed by calculating the peak area ratio of each unlabeled peak area to the corresponding internal standard peak area. Chromatograms were acquired and processed with Masslynx V4.1 SCN 644 (Waters, M ilford, USA). 


\section{Method validation}

Linearity was determined by adding standard solution of CM L, CEL and M G-H1 to water and selected food matrix and subsequently hydrolyzed. To investigate acid stability of $\mathrm{CML}, \mathrm{CEL}$ and $\mathrm{MG}-\mathrm{H} 1$ these results were compared to a calibration in water without acid hydrolysis. A six-point calibration curve was therefore prepared for CM L $(0-5250 \mathrm{nmol} / \mathrm{L})$, CEL $(0-6250 \mathrm{nmol} / \mathrm{L})$ and MG-H1 $(0-14750 \mathrm{nmol} / \mathrm{L})$. The peak area ratio of the analyte divided by the internal standard area of $\mathrm{CML}, \mathrm{CEL}$ and $\mathrm{MG}-\mathrm{H} 1$ multiplied by the concentration of each corresponding internal standard ( $y$, response factor in $\mathrm{nmol} / \mathrm{L}$ ) were plotted as a function of the analyte concentration ( $\mathrm{x}$, concentration in $\mathrm{nmol} / \mathrm{L}$ ). The intra-assay variation was determined in three different food items, containing low, medium and high concentration of AGEs, analyzed in one batch during one day $(n=8)$. The inter-assay variation was determined in three different food items divided into batches and analyzed on three different days. To investigate reproducibility, intra-product, intraand inter-brand variations in different food items a selection of foods, containing both high and low protein content and/or fat percentage, was analyzed in triplicate.

To determine extraction differences between TFA and chloroform:methanol (2:1, v/v) extraction, a milk, white bread and ketchup sample was deproteinized in triplicate, using both extraction fluids. AGE content in these selected food items, contained by both extraction fluids, was compared.

\section{Preparation of minced-meat hot dog}

To determine the extent of AGE-formation during preparation of minced-meat hot dog, a standard serving size of minced-meat hot dog was deep fried at a temperature of $190^{\circ} \mathrm{C}$. AGE-content was determined in an unbaked minced-meat hot dog ( $\mathrm{t}=0$ minutes) and after 2, 4 and 6 minutes of deep-frying.

\section{Results}

\section{Reduction of food samples}

To prevent neo-formation of CM L during acid hydrolysis a reduction step was used. The effectiveness of the reduction step with sodium borohydride was tested with different sodium borohydride concentrations and different incubation times. No further decrease in $\mathrm{CML}$ concentration was observed with a reduction step with sodium borohydride concentrations $>100 \mathrm{mmol} / \mathrm{L}$ for 2 hours at room temperature (figure 8.1A). Under the same conditions, CEL and M G-H1 concentrations were also (figure 8.1A).

No further decrease in $\mathrm{CML}, \mathrm{CEL}$ and $\mathrm{MG}-\mathrm{H} 1$ was observed using an incubation time longer than 2 hours (figure 8.1B). Therefore, a reduction step for 2 hours with a concentration of $100 \mathrm{mmol}$ sodium borohydride/ L was used for all food samples. 
(A)

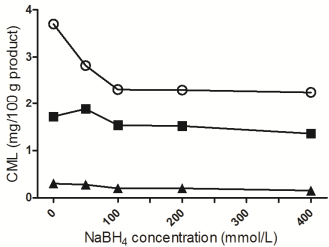

(B)

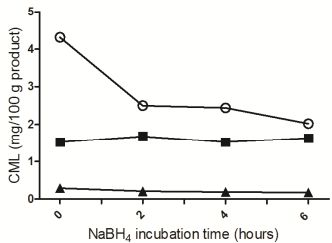

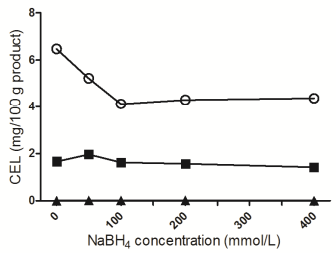
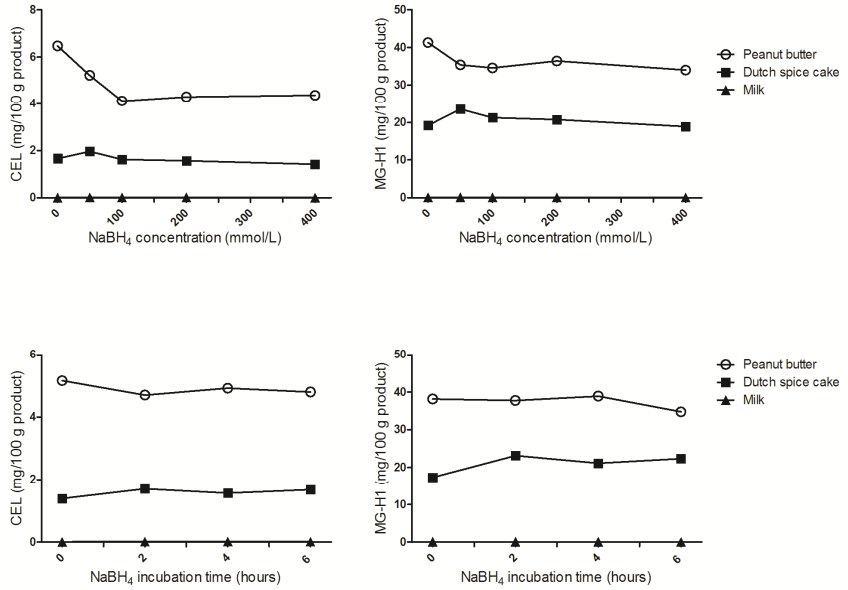

Figure 8.1 The effect of $\mathrm{NaBH} 4$ concentration (A) and incubation time (B) on the concentration of CM L, CEL and M G-H1 as analyzed in a peanut butter, a Dutch spice cake and a milk sample. Reduction of food samples for 2 hours with $0,50,100,200$ and $400 \mathrm{mmol} / \mathrm{L} \mathrm{NaBH} 4$ (A) and reduction of food samples with a concentration of $100 \mathrm{mmol} / \mathrm{L} \mathrm{NaBH} 4$ for 0, 2, 4 and 6 hours (B).

\section{Method validation}

The calibration curves for CML, CEL and MG-H1 were linear over the described concentration ranges $\left(r^{2} ; 0.90-0.99\right)$ in both water and food matrices. Mean slopes (response factor) for CML, CEL and M G-H1 tested in 3 different matrices were 1.13 (CV, $4.4 \%), 1.65$ (CV, 6.1\%) and 0.55(CV, 6.8\%), respectively (figure 8.2).
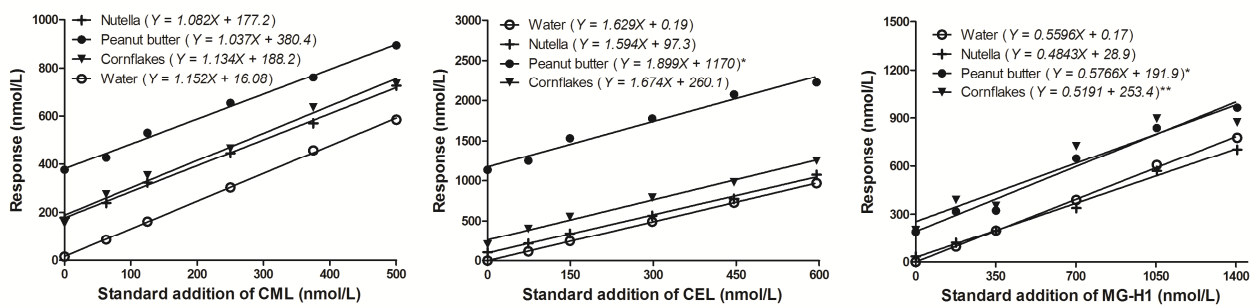

Figure 8.2 Standard addition of CM L, CEL and M G-H1 to water, peanut butter, Nutella and Cornflakes.

Linearity was determined by adding standard solution of CML, CEL and MG-H1 to water and selected food matrix (peanut butter, Nutella and Cornflakes) and subsequently hydrolyzed.

The peak area ratio of the analyte/internal standard area of CML, CEL and MG-HI multiplied by the concentration of each corresponding internal standard (y) were plotted as a function of the analyte concentration (x). Calibration curves showed perfect linearity $\left(r^{2}>0.99, * r^{2}=0.98\right.$ and $\left.* * r^{2}=0.90\right)$.

Impurities coming from the stable isotope internal standard or caused by memory effect of the auto-injector were negligible since calibration curves in water showed no or low $y$-axis intercepts. Inter-assay variation of CML, CEL and MG-H1, as determined by replicate analysis of a peanut butter, a $M$ ars and a Cornflakes sample on three different days, and intra-assay variation, as determined by replicate analysis $(n=8)$ of these food items on one day, were between 1.7 and $29.1 \%$ (table 8.1). 
The mean limit of quantification (LOQ), as determined in a peanut butter, a $\mathrm{M}$ ars and a Cornflakes sample at a signal-to-noise ratio of $10(\mathrm{~s} / \mathrm{N}=10)$ for $\mathrm{CML}, \mathrm{CEL}$ and $\mathrm{M} \mathrm{G-H1}$ were 1,1 and $25 \mathrm{fmol}$ on column, respectively. These LOQ's were equivalent to $0.02,0.02$ and $0.5 \mathrm{mg}$ of CM L, CEL and M G-H1 per $100 \mathrm{~g}$ product, respectively.

We applied two different protein precipitation methods for protein pellet collection: 1) Food items containing $<20 \%$ fat were precipitated with TFA and 2) food items containing $>20 \%$ fat were precipitated with chloroform:methanol $(2: 1, v / v)^{37}$.

When we compared both precipitation methods in a milk, white bread and ketchup sample, we found approximately $82 \%$ recovery with the chloroform:methanol denaturation as compared to TFA.

Table 8.1 Inter- and intra-assay variation of CML, CEL and MG-H1 as tested in a peanut butter, Mars and Cornflakes sample. Data are expressed as mean AGE mg/ 100 g product (CV, \%).

\begin{tabular}{|c|c|c|c|c|c|c|}
\hline & \multicolumn{2}{|c|}{ CML mean (CV, \%) } & \multicolumn{2}{|c|}{ CEL mean (CV, \%) } & \multicolumn{2}{|c|}{ MG-H1 mean (CV, \%) } \\
\hline & $\begin{array}{l}\text { Inter-run } \\
(n=3)\end{array}$ & $\begin{array}{l}\text { Intra-run } \\
(n=8)\end{array}$ & $\begin{array}{l}\text { Inter-run } \\
(n=3)\end{array}$ & $\begin{array}{l}\text { Intra-run } \\
(n=8)\end{array}$ & $\begin{array}{l}\text { Inter-run } \\
(n=3)\end{array}$ & $\begin{array}{l}\text { Intra-run } \\
(n=8)\end{array}$ \\
\hline Peanut butter & $1.48(7.0)$ & $1.38(5.1)$ & $3.84(4.1)$ & $3.74(6.7)$ & $18.69(17.9)$ & $20.39(25.0)$ \\
\hline Mars & $0.67(15.0)$ & $0.70(4.7)$ & $0.33(9.8)$ & $0.36(6.0)$ & $0.14(16.8)$ & $0.15(18.3)$ \\
\hline Cornflakes & $0.72(7.7)$ & $0.66(12.8)$ & $0.84(6.7)$ & $0.79(16.2)$ & $12.27(1.7)$ & $12.12(29.1)$ \\
\hline
\end{tabular}

\section{Acid stability of AGEs}

The calibration curves for CML, CEL and M G-H1, as tested in water after acid hydrolysis, were compared to the calibration curves without acid hydrolysis. The slope for CM L, CEL and MG-H1 as tested in water, without acid hydrolysis, was 1.18, 1.59 and 1.04, respectively. For CML and CEL no differences were observed between the mean slopes of the calibration curve with or without acid hydrolysis. For M G-H1, however, a decrease of the mean slope from 1.03 to 0.56 was observed after acid hydrolysis. Thus, under these standard conditions we found a recovery of MG-H1 of $\sim 55 \%$.

\section{AGE formation during preparation of minced-meat hot dog}

To further investigate the formation of AGEs during food heating, minced-meat hot dog was fried at a temperature of $190^{\circ} \mathrm{C}$ in frying fat. Unbaked minced-meat hot dog contained $0.28,0.10$ and $3.30 \mathrm{mg} \mathrm{CML}, \mathrm{CEL}$ and M G-H1 per $100 \mathrm{~g}$ product, respectively. After 2, 4 and 6 minutes of frying, samples were collected and the concentrations of all three AGEs increased in a time-dependent manner (figure 8.3). After 6 minutes of deepfrying $\mathrm{CML}, \mathrm{CEL}$ and MG-H1 were increased to 4.82, 11.35 and $33.64 \mathrm{mg}$ per $100 \mathrm{~g}$ product, respectively. 


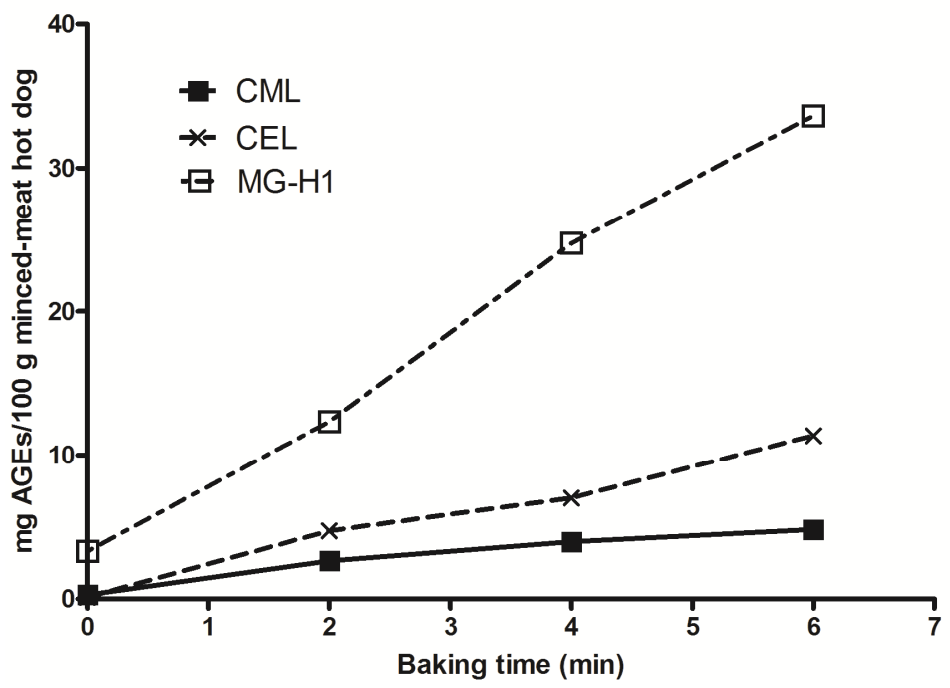

Figure 8.3 Formation of AGEs in deep fried minced-meat hot dog. AGE-formation during preparation of mincedmeat hot dog during deep frying at a temperature of $190^{\circ} \mathrm{C}$. AGE-content was determined in an unbaked minced-meat hot dog ( $t=0$ minutes) and after 2, 4 and 6 minutes of deep-frying.

\section{AGE content in selected food items}

The complete list of AGE levels in the selected food items is presented in table s8.1 (supplemental table). Black pudding, cereals (miniwheats, frosted flakes, cornflakes and puffed wheat), peanut butter, peanuts, rusk and biscuits (with peanuts, speculoos and multigrain), contained high levels of CML, CEL and MG-H1 (supplemental table s8.1). Overall the food items which were prepared by an exposure to heat and long processing times (such as black pudding fried for 16 minutes or red cooked beefstewed for 2.5 hours in wine vinegar) or baked (such as biscuits, cake and knäckebröd) or industrial conservation (such as canned products which were commercially sterilized at $115-130^{\circ} \mathrm{C}$ for 15 minutes) or products containing chocolate, nuts or grains were high in AGE content.

Food items with high AGE content varied from $\sim 2$ to $\sim 5 \mathrm{mg} \mathrm{CM} \mathrm{L,} \mathrm{from} \sim 2$ to $\sim 7 \mathrm{mg}$ CEL and from $\sim 15$ to $\sim 60 \mathrm{mg} \mathrm{M} \mathrm{G-H1}$ per $100 \mathrm{~g}$ product. Coffee, fruits, vegetables, butter, olive oil and red wine had negligible AGE content. Overall, the food items with a short heat processing time, low protein and/or carbohydrate content or high water content had low or negligible levels of CM L, CEL and M G-H1. 


\section{Discussion}

Here, we describe a sensitive, precise and well-validated UPLC-M S/MS method for the quantification of the AGES CM L, CEL and M G-H1 in a broad range of different food items. We report a database of CML, CEL and MG-H1 in 190 food items. We found high AGE levels in food items which had been prepared by an exposure to heat, industrial conserving or products containing chocolate, nuts or grains, whereas food products with short or no heat processing, low protein and/or carbohydrate content, or high water content had low or negligible AGE content.

The protein-bound AGE concentrations in foods have previously been analyzed by different sample preparation techniques. Depending on the acid stability of the AGE of interest, the protein-bound AGE content was determined by means of hydrochloric acid hydrolysis ${ }^{14,15,27,33,38}$ or by enzymatic hydrolysis using a cocktail of proteases ${ }^{13,29,38}$. In our study CM L and CEL proved to be acid stable, but M G-H1 showed a reproducible decrease of approximately $45 \%$ after acid hydrolysis. Therefore, the M G-H1 content was adjusted for this decrease. This decrease in MG-H1 was in agreement with previously reported data ${ }^{39}$, but we found that the order of magnitude was less than the approximately $90 \%$ reduction in hydroimidazolones after acid hydrolysis which was previously reported. This difference may be explained by sample preparation differences, such as the addition of nitrogen, phenol and/or mercaptoethanol during acid hydrolysis. With an inter- and intraassay variation of approximately $10 \%$, linear and reproducible calibration curves in both water and food matrix and a high specificity and sensitivity, the analysis proved to have good validity.

In the present study peanuts, peanut butter, rusk, biscuits, cereals, toast and high-heatprocessed meats, such as red cooked beef, canned beef steak and black pudding, had the highest levels of CML, CEL and M G-H1, while coffee, fruits, vegetables, butter, olive oil and red wine had negligible AGE content. For $C M L$ these findings were in accordance with data described in literature ${ }^{27}$ although absolute levels of $C M L$ content differ. Food preparation techniques, such as pasteurization, sterilization, roasting, baking, frying or boiling, could strongly influence the AGE content ${ }^{13-15,29,33}$ and therefore comparison of absolute AGE levels between studies is difficult. Moreover, differences between the ingredient profile, sample preparation or quantitative methodology should also be considered.

AGE formation during heat processing, industrial cooking or cooking in sauce, as observed in many studies ${ }^{13,14,26,27,29,30,33,38}$, was confirmed in our study. We observed an increase in AGE content in minced-meat hot dog during frying. Moreover, high AGE levels were observed in red cooked beef, which was stewed in wine vinegar sauce. We also found a 5to 10 -fold increase in AGE content in semi-skimmed evaporated milk compared to semiskimmed milk and these results were in good accordance with Hegele et al. ${ }^{38}$ and Assar et al. ${ }^{33}$ who observed that milk products which had undergone extensive industrial processing, such as condensed or evaporated milk, were high in AGE content. 
In general, high heat treatment or a long treatment time in canning or stewing is most likely responsible for generating elevated AGE levels in food.

So far, three major studies have described the CML content in a large selection of food items $s^{25-27}$ and one major study investigated four AGEs, including $C M L$, in beverages and foods commonly consumed in Japan ${ }^{28}$. Three of these studies were based on ELISA technique $\mathrm{e}^{25,26,28}$ and one study investigated CM L content in foods using UPLC-tandem MS analysis ${ }^{27}$.

In the present study we found low or negligible CML content in mayonnaise, butter and olive oil which was in agreement with the findings of Hull et al. ${ }^{27}$ using an UPLC-MS/MS technique. Differences in the CML content of a Mars and Bounty candy bar were observed. We found approximately a 2-fold lower CML content in Mars and a 10-fold lower CML content in a Bounty candy bar as compared to the results of Hull et al. ${ }^{27}$. Moreover, we found a 6-fold lower CML content in whole meal bread, Kellog's corn flakes, white rice, parmesan cheese and canned salmon as compared to their results. These differences may be explained by differences in sample preparation or ingredient profile.

Using an ELISA technique, Uribarri et al. ${ }^{26}$ found high AGE levels in all products containing fat such as butter, olive oil, cheese and milk products and also in most meats but not in food items that are expected to contain high AGE levels such as biscuits, crackers and cookies. In contrast, we found that most cheese and milk products are low in AGE content (with the exception of evaporated milk). Indeed, previous studies show also inconsistencies in $C M L$ content in foods when analyzed by mass spectrometry or ELISA $^{24,40}$. These differences might be caused by an unspecific interference of the lipid matrix with the ELISA ${ }^{24}$ since ELISA techniques have a high risk of matrix interference as the antigen-antibody interaction is dependent on the chemical environment ${ }^{16}$.

The main advantages of the present study above the previously described studies are the use of a state-of-the-art UPLC-tandem MS technique and the ability to analyze three major AGEs in one run.

To further investigate the relevance of dietary AGEs in disease, the presented reliable and valid analytical method is suitable for the analysis of CML, CEL and MG-H1 in selected food items and is a strong tool to build up an extensive dietary AGE database. The present dietary AGE database will be valuable for designing intervention studies with low and high AGE diets to study the health effect of AGEs in much more detail than has hitherto been possible. 


\section{References}

1

2

Brownlee, M., Vlassara, H. \& Cerami, A. Nonenzymatic glycosylation and the pathogenesis of diabetic complications. Annals of internal medicine 101, 527-537 (1984).

$$
\text { Monnier, V. M. \& Cerami, A. Nonenzymatic browning in vivo: possible process for aging of long-lived }
$$
proteins. Science 211, 491-493 (1981).

Maillard, L. C. Action des acides aminés sur les sucres: formation des mélanoidines par voie méthodique. C.R. Acad. Sci. 154, 66-68 (1912).

Basta, G., Schmidt, A. M. \& De Caterina, R. Advanced glycation end products and vascular inflammation: implications for accelerated atherosclerosis in diabetes. Cardiovasc Res 63, 582-592 (2004).

Baumann, M. et al. N epsilon-(carboxymethyl)lysine during the early development of hypertension. Ann N Y Acad Sci 1126, 201-204 (2008).

Goldin, A., Beckman, J. A., Schmidt, A. M \& \& Creager, M. A. Advanced glycation end products: sparking the development of diabetic vascular injury. Circulation 114, 597-605 (2006).

Nin, J. W. et al. Higher plasma levels of advanced glycation end products are associated with incident cardiovascular disease and all-cause mortality in type 1 diabetes: a 12-year follow-up study. Diabetes Care 34, 442-447 (2011).

Bierhaus, A. et al. Diabetes-associated sustained activation of the transcription factor nuclear factorkappaB. Diabetes 50, 2792-2808 (2001).

Giardino, I., Edelstein, D. \& Brownlee, M. Nonenzymatic glycosylation in vitro and in bovine endothelial cells alters basic fibroblast growth factor activity. A model for intracellular glycosylation in diabetes. The Journal of clinical investigation 94, 110-117, doi:10.1172/JCl117296 (1994).

Gaens, K. H., Stehouwer, C. D. \& Schalkwijk, C. G. Advanced glycation endproducts and its receptor for advanced glycation endproducts in obesity. Curr Opin Lipidol 24, 4-11, doi:10.1097/M OL.0b013e32835aea13 (2013).

1 Fu, M. X. et al. The advanced glycation end product, Nepsilon-(carboxymethyl)lysine, is a product of both lipid peroxidation and glycoxidation reactions. The Journal of biological chemistry $\mathbf{2 7 1}$, $9982-$ 9986 (1996).

2 Kislinger, T. et al. N(epsilon)-(carboxymethyl)lysine adducts of proteins are ligands for receptor for advanced glycation end products that activate cell signaling pathways and modulate gene expression. The Journal of biological chemistry 274, 31740-31749 (1999).

3 Zhang, G., Huang, G., Xiao, L. \& Mitchell, A. E. Determination of advanced glycation endproducts by LC-M S/M S in raw and roasted almonds (Prunus dulcis). Journal of agricultural and food chemistry 59, 12037-12046, doi:10.1021/jf202515k (2011).

4 Pei-Chun Chao, C.-c. H., Mei-chin Yin. Analysis of glycative products in sauces and sauce-treated foods. Food Chemistry 113, 262-266 (2009).

5 Wellner, A., Huettl, C. \& Henle, T. Formation of Maillard reaction products during heat treatment of carrots. Journal of agricultural and food chemistry 59, 7992-7998, doi:10.1021/jf2013293 (2011). Poulsen, M. W. et al. Advanced glycation endproducts in food and their effects on health. Food Chem Toxicol 60, 10-37, doi:10.1016/j.fct.2013.06.052 (2013). 
Vlassara, $\mathrm{H}$. et al. Exogenous advanced glycosylation end products induce complex vascular dysfunction in normal animals: a model for diabetic and aging complications. Proc Natl Acad Sci U SA 89, 12043-12047 (1992).

Guo, Q. et al. Methylglyoxal contributes to the development of insulin resistance and salt sensitivity in Sprague-Dawley rats. J Hypertens 27, 1664-1671, doi:10.1097/HJH.0b013e32832c419a (2009). Uribarri, J. et al. Single oral challenge by advanced glycation end products acutely impairs endothelial function in diabetic and nondiabetic subjects. Diabetes Care 30, 2579-2582, doi:10.2337/dc07-0320 (2007).

Uribarri, J. et al. Restriction of advanced glycation end products improves insulin resistance in human type 2 diabetes: potential role of AGER1 and SIRT1. Diabetes Care 34, 1610-1616, doi:10.2337/dc110091 (2011).

Uribarri, J. et al. Diet-derived advanced glycation end products are major contributors to the body's AGE pool and induce inflammation in healthy subjects. Annals of the New York Academy of Sciences 1043, 461-466, doi:10.1196/annals.1333.052 (2005).

Birlouez-Aragon, I. et al. A diet based on high-heat-treated foods promotes risk factors for diabetes mellitus and cardiovascular diseases. Am J Clin Nutr 91, 1220-1226, doi:10.3945/ajcn.2009.28737 (2010).

Mark, A. B. et al. Consumption of a Diet Low in Advanced Glycation Endproducts for 4 weeks Improves Insulin Sensitivity in Overweight Women. Diabetes Care, doi:10.2337/dc13-0842 (2013). Charissou, A., Ait-Ameur, L. \& Birlouez-Aragon, I. Evaluation of a gas chromatography/mass spectrometry method for the quantification of carboxymethyllysine in food samples. J Chromatogr A 1140, 189-194, doi:10.1016/j.chroma.2006.11.066 (2007).

Goldberg, T. et al. Advanced glycoxidation end products in commonly consumed foods. Journal of the American Dietetic Association 104, 1287-1291, doi:10.1016/j.jada.2004.05.214 (2004).

Uribarri, J. et al. Advanced glycation end products in foods and a practical guide to their reduction in the diet. Journal of the American Dietetic Association 110, $911-916$ e912, doi:10.1016/j.jada.2010.03.018 (2010).

Hull, G. L. J., Woodside, J. V., Ames, J. M . \& Cuskelly, G. J. Nع-(carboxymethyl)lysine content of foods commonly consumed in a Western style diet. Food Chemistry 131, 170-174 (2012).

Takeuchi, M . et al. Assessment of the concentrations of various advanced glycation end-products in beverages and foods that are commonly consumed in Japan. PLOS One 10, e0118652, doi:10.1371/journal.pone.0118652 (2015).

Ahmed, N. et al. Assay of advanced glycation endproducts in selected beverages and food by liquid chromatography with tandem mass spectrometric detection. M olecular nutrition \& food research $\mathbf{4 9}$, 691-699, doi:10.1002/mnfr.200500008 (2005).

Chen, G. \& Smith, J. S. Determination of advanced glycation endproducts in cooked meat products. Food Chem 168, 190-195, doi:10.1016/j.foodchem.2014.06.081 (2015). carboxymethyllysine in rats. Amino acids 44, 1441-1449, doi:10.1007/s00726-012-1427-3 (2013). determination of early and advanced glycation-induced lysine modifications. Ann N Y Acad Sci 1126, 300-306 (2008). 
33

34

35

36

37

38

39

40

Assar, S. H., Moloney, C., Lima, M., Magee, R. \& Ames, J. M. Determination of Nepsilon(carboxymethyl)lysine in food systems by ultra performance liquid chromatography-mass spectrometry. Amino acids 36, 317-326, doi:10.1007/s00726-008-0071-4 (2009).

34 Spanneberg, R., Salzwedel, G. \& Glomb, M. A. Formation of early and advanced Maillard reaction products correlates to the ripening of cheese. Journal of agricultural and food chemistry $60,600-607$, doi:10.1021/jf204079f (2012).

35 Ocke, M. C. et al. The Dutch EPIC food frequency questionnaire. I. Description of the questionnaire, and relative validity and reproducibility for food groups. Int J Epidemiol 26 Suppl 1, S37-48 (1997).

36 Hulshof K F A M, v. S. W. A. The Dutch National Food Consumption Survey: design, methods and first results. Food Policy 16, 257-260 (1991).

Folch, J., Lees, M. \& Sloane Stanley, G. H. A simple method for the isolation and purification of total lipides from animal tissues. The Journal of biological chemistry 226, 497-509 (1957).

38 Hegele, J., Buetler, T. \& Delatour, T. Comparative LC-M S/MS profiling of free and protein-bound early and advanced glycation-induced lysine modifications in dairy products. Anal Chim Acta 617, 85-96 (2008).

39 Ahmed, N., Argirov, O. K., Minhas, H. S., Cordeiro, C. A. \& Thornalley, P. J. Assay of advanced glycation endproducts (AGEs): surveying AGEs by chromatographic assay with derivatization by 6 aminoquinolyl-N-hydroxysuccinimidyl-carbamate and application to Nepsilon-carboxymethyl-lysineand Nepsilon-(1-carboxyethyl)lysine-modified albumin. Biochem J 364, 1-14 (2002).

Delatour, T. et al. Analysis of advanced glycation endproducts in dairy products by isotope dilution liquid chromatography-electrospray tandem mass spectrometry. The particular case of carboxymethyllysine. J Chromatogr A 1216, 2371-2381 (2009). 
160 Chapter 8 


\section{Appendix 8 Supplemental table}




\section{Supplemental table 8}

Table s8.1 CM L, CEL and M G-H1 content in food (* content in $\mathrm{mg} / 100 \mathrm{~g}$ food).

Potatoes

\begin{tabular}{llllll}
\hline & Food & Specification & CML* & CEL* & M G-H1* \\
\hline 1 & Chips & Deep fried 5 minutes & 0.14 & 0.09 & 1.28 \\
2 & Potato-boiled & Boiled, 7 minutes & 0.01 & 0 & 0.39 \\
3 & Potato-fried & Boiled, 7 minutes, fried 6 minutes & 0.01 & 0.01 & 0.3 \\
\hline
\end{tabular}

Alcoholic and non-alcoholic beverages

\begin{tabular}{llllll}
\hline & Food & Specification & CML* & CEL* & M G-H1* \\
\hline 4 & Coffee & & 0 & 0.01 & 0.24 \\
5 & Red wine & Kiwi/Orange & 0 & 0 & 0 \\
6 & Fruit juice & & 0 & 0 & 0.05 \\
7 & Cola & 0 & 0 & 0 \\
\hline
\end{tabular}

\begin{tabular}{clllll}
\multicolumn{2}{c}{ Eggs } & \multicolumn{2}{l}{} \\
\hline \multicolumn{2}{c}{ Food } & Specification & CML* & CEL* & M G-H1* \\
\hline 8 & Egg yolk & Boiled, 10 minutes & 0.06 & 0.08 & 0.08 \\
9 & Egg white & Boiled, 10 minutes & 0.1 & 0.07 & 0.35 \\
10 & Fried egg & Fried, 4 minutes & 0.42 & 0.52 & 4.5 \\
\hline
\end{tabular}

\section{Fruits}

\begin{tabular}{llllll}
\hline & Food & Specification & CM L* & CEL* & M G-H1* \\
\hline 11 & Kaki & & 0 & 0 & 0 \\
12 & Banana & & 0.01 & 0 & 0.05 \\
13 & Apple sauce & Light & 0 & 0 & 0.2 \\
14 & Olives & Green & 0.12 & 0.01 & 1.54 \\
\hline
\end{tabular}

\section{Vegetables}

\begin{tabular}{|c|c|c|c|c|c|}
\hline & Food & Specification & CML* & CEL* & M G-H1* \\
\hline 15 & Mushroom & Fried, 5 minutes & 0.01 & 0.01 & 0.01 \\
\hline 16 & Peas & Boiled & 0.05 & 0.06 & 2.31 \\
\hline 17 & Tomato & Fried, 3 minutes & 0.07 & 0.05 & 0.65 \\
\hline 18 & Tomato, canned & & 0.03 & 0.01 & 0.11 \\
\hline
\end{tabular}

Savoury bread spreads

\begin{tabular}{llllll}
\hline & Food & Specification & CML* & CEL* & M G-H1* \\
\hline 19 & $\begin{array}{l}\text { Peanut butter } \\
(n=3)^{* *}\end{array}$ & Pâte de cacahouètes, 72\% peanut & $1.91 \pm 0.23$ & $5.56 \pm 0.31$ & $35.62 \pm 2.51$ \\
20 & $\begin{array}{l}\text { Peanut butter } \\
(n=3)^{* *}\end{array}$ & Delvita Erdnuss Crème, 90\% peanut & $3.11 \pm 0.31$ & $6.74 \pm 0.45$ & $44.52 \pm 2.03$ \\
21 & $\begin{array}{l}\text { Peanut butter } \\
(n=3)^{* *}\end{array}$ & Calvé, 85\% peanut & $2.63 \pm 0.68$ & $6.85 \pm 0.68$ & $42.05 \pm 4.08$ \\
22 & $\begin{array}{l}\text { Peanut butter } \\
(n=8)\end{array}$ & Plus, 90\% peanut & $1.38 \pm 0.07$ & $3.74 \pm 0.25$ & $20.39 \pm 5.09$ \\
\hline
\end{tabular}


Cheese

\begin{tabular}{llllll}
\hline \multicolumn{2}{l}{ Food } & Specification & CML* & CEL* & M G-H1* \\
\hline 23 & Cheese & Gouda 48+(32\% fat) & 0.03 & 0.01 & 0.08 \\
24 & Cheese & (32\% fat) & 0.04 & 0.08 & 0.25 \\
25 & Cheese & Old (35\% fat) & 0.08 & 0.22 & 0.21 \\
26 & Parmesan cheese & Parmigiano Reggiano (28\% fat) & 0.22 & 0.63 & 2.76 \\
27 & Cheese & (32\% fat) & 0.15 & 0.55 & 0.3 \\
28 & Cream cheese & (7\% fat) & 0.35 & 0.08 & 0.49 \\
29 & French cheese & Brie, Coeur de Lion, 60+(32\% fat) & 0.01 & 0 & 0.07 \\
30 & French cheese & Camembert Chêne d'argent (21\% fat) & 0.12 & 0.3 & 0.16 \\
31 & Cottage cheese & & 0.05 & 0.04 & 0.12 \\
32 & Mozzarella & & 0.07 & 0.01 & 0.13 \\
33 & Feta cheese & & 0.08 & 0.47 & 1.14 \\
\hline
\end{tabular}

Milk and milk products

\begin{tabular}{|c|c|c|c|c|c|}
\hline & Food & Specification & CML* & CEL* & MG-H1* \\
\hline 34 & Milk & Semi-skimmed & 0.25 & 0.02 & 0.12 \\
\hline 35 & Milk & Skimmed & 0.07 & 0.01 & 0.08 \\
\hline 36 & Milk & Skimmed & 0.26 & 0.02 & 0.3 \\
\hline 37 & Milk & Skimmed, microwave 2 min & 0.36 & 0.02 & 0.38 \\
\hline 38 & Yoghurt drink & $0 \%$ fat, raspberry & 0.04 & 0.03 & 0.12 \\
\hline 39 & "Kwark" & Curd, skimmed & 0.06 & 0.04 & 0.26 \\
\hline 40 & Milk & Whole & 0.01 & 0.01 & 0.02 \\
\hline 41 & Evaporated milk & & 0.66 & 0.03 & 0.09 \\
\hline 42 & Evaporated milk & Skimmed & 0.47 & 0.08 & 0.38 \\
\hline 43 & Evaporated milk & Semi-skimmed & 2.23 & 0.12 & 0.54 \\
\hline 44 & Whipped cream & $35 \%$ fat & 0 & 0 & 0 \\
\hline 45 & Chocolate milk & Whole & 0.38 & 0.2 & 0.66 \\
\hline 46 & Chocolate milk & Semi-skimmed & 1.01 & 0.26 & 0.98 \\
\hline 47 & Custard & "Vla", chocolate/vanilla & 0.12 & 0.06 & 0.43 \\
\hline 48 & Porridge & Rolled oats & 0.02 & 0 & 0.06 \\
\hline 49 & Porridge & Rice & 0.03 & 0.02 & 0.21 \\
\hline 50 & Pudding & Raspberry, with strawberry sauce & 0.26 & 0.02 & 0.33 \\
\hline 51 & Yogurt & Semi-skimmed, vanilla & 0.01 & 0.01 & 0.13 \\
\hline 52 & Ice-cream & Vanilla/Strawberry & 0 & 0 & 0.03 \\
\hline
\end{tabular}

Soy products and vegetarian products

\begin{tabular}{llllll}
\hline & Food & Specification & CML* & CEL* & M G-H1* \\
\hline 53 & Vegetarian burger & Vegetable burger, fried 5 minutes & 0.42 & 0.6 & 3.6 \\
54 & Tofu & Fried 10 minutes & 0.94 & 0.74 & 11.87 \\
55 & Tempe & Boiled, 10 minutes & 0.47 & 0.29 & 3.5 \\
56 & Tempe & Grilled, 4 minutes & 1 & 0.59 & 6.87 \\
57 & Soy sauce & Ketjap manis & 0.01 & 0.02 & 0.05 \\
\hline
\end{tabular}


Sugar, sweets and sweet sauces

\begin{tabular}{llllll}
\hline & Food & Specification & CM L* & CEL* & M G-H $1^{*}$ \\
\hline 58 & Praline & With orange crème filling & 0.41 & 0.09 & 0.87 \\
59 & Chocolate & White & 0.52 & 0.06 & 0.42 \\
60 & Chocolate & Milk & 0.96 & 0.31 & 1.33 \\
61 & Chocolate & Milk, with hazelnuts & 1.33 & 0.55 & 3.23 \\
62 & Chocolate & Dark & 3.51 & 1.28 & 3.03 \\
63 & Mars (n=8) & & $0.70 \pm 0.03$ & $0.36 \pm 0.02$ & $0.15 \pm 0.03$ \\
64 & Twix & & 0.93 & 0.81 & 5.83 \\
65 & Bounty & & 0.71 & 0.23 & 2.16 \\
66 & Milky Way & & 1.68 & 0.56 & 0.54 \\
67 & Liquorice & & 0.06 & 0.02 & 0.08 \\
68 & Candy & Jelly bears & 0.03 & 0.02 & 0.11 \\
69 & Jam & Blackberry & 0 & 0 & 0 \\
70 & "Hagelslag" & Chocolate sprinkles & 5.09 & 2.03 & 9.33 \\
71 & "Hagelslag" & Fruit sprinkles & 0 & 0 & 0 \\
72 & "Stroop" & Apple syrup & 0.05 & 0.02 & 0.44 \\
73 & Chocolate spread & & 0.9 & 0.34 & 3.27 \\
\hline
\end{tabular}

Fats, oils and savoury sauces

\begin{tabular}{|c|c|c|c|c|c|}
\hline & Food & Specification & CML* & CEL* & M G-H1* \\
\hline 74 & Peanut sauce & $\begin{array}{l}\text { M icrowave heated, } 0.5 \text { minutes, } \\
800 \text { Watt }\end{array}$ & 0.21 & 0.33 & 1.9 \\
\hline 75 & Peanut sauce & $\begin{array}{l}\text { Microwave heated, } 1.5 \text { minutes, } \\
800 \text { Watt }\end{array}$ & 0.65 & 0.95 & 6.94 \\
\hline 76 & Peanut sauce & $\begin{array}{l}\text { Instant, } 90 \% \text { peanut, 1:1 made up } \\
\text { with boiling water }\end{array}$ & 1.29 & 2.35 & 14.03 \\
\hline 77 & Peanut sauce & Pan heated, $500 \mathrm{~g}$ with $300 \mathrm{ml}$ water & 0.8 & 1.39 & 12.6 \\
\hline 78 & Tomato sauce & Pan heated & 0.04 & 0.05 & 0.22 \\
\hline 79 & Curry sauce & $\begin{array}{l}\text { Instant, 1:10 made up with boiling } \\
\text { water }\end{array}$ & 0.05 & 0.01 & 0.13 \\
\hline 80 & Cheese-sauce & $\begin{array}{l}\text { Instant, 1:6 made up with boiling } \\
\text { water }\end{array}$ & 0.06 & 0.02 & 0.18 \\
\hline 81 & Mayonnaise & & 0.01 & 0 & 0.12 \\
\hline 82 & Frying butter & Liquid, pan heated & 0 & 0 & 0 \\
\hline 83 & Butter & Diet (60\% fat) & 0 & 0 & 0 \\
\hline 84 & Olive oil & Pan heated & 0 & 0 & 0 \\
\hline 85 & Ketchup & & 0.05 & 0.01 & 0.05 \\
\hline 86 & Garlic sauce & & 0.01 & 0 & 0.02 \\
\hline
\end{tabular}


Fish

\begin{tabular}{|c|c|c|c|c|c|}
\hline & Food & Specification & CML* & CEL* & MG-H1* \\
\hline 87 & Salmon, canned & In brine & 1.17 & 2.82 & 10.93 \\
\hline 88 & Salmon, fried & Fried, 10 minutes & 0.41 & 0.58 & 1.83 \\
\hline 89 & Salmon, smoked & & 0.58 & 0.22 & 0.32 \\
\hline 90 & Herring & Raw & 0.06 & 0.06 & 0.09 \\
\hline 91 & Hake & $\begin{array}{l}\text { Breaded, oven heated, } 10 \text { minutes } \\
200^{\circ} \mathrm{C}\end{array}$ & 0.38 & 0.31 & 0.55 \\
\hline 92 & Whitefish & Grilled, in soy sauce & 0.26 & 0.29 & 1.08 \\
\hline 93 & Prawns & Fried, 5 minutes & 0.06 & 0.07 & 1.03 \\
\hline 94 & Tuna, canned & In olive oil & 0.35 & 1.26 & 3.78 \\
\hline 95 & Cod & Fillet, fried 8 minutes & 0.13 & 0.11 & 0.66 \\
\hline 96 & Steamed fish & Oven heated, 15 minutes $175^{\circ} \mathrm{C}$ & 0.05 & 0.06 & 0.11 \\
\hline 97 & Fish fingers & Oven heated, 15 minutes $175^{\circ} \mathrm{C}$ & 0.3 & 0.18 & 4.3 \\
\hline
\end{tabular}

Nuts, seeds and snacks

\begin{tabular}{|c|c|c|c|c|c|}
\hline & Food & Specification & CML* & CEL* & M G-H1* \\
\hline 98 & Pistachios & Unprocessed & 0.21 & 0.09 & 0.32 \\
\hline 99 & Pecans & Roasted, salted & 1.06 & 1.95 & 16.63 \\
\hline 100 & Peanuts & In shell & 1.37 & 2.43 & 25.71 \\
\hline 101 & Peanuts & Salted & 1.72 & 3.39 & 26.59 \\
\hline 102 & Cocktail nuts & "Borrelnoten" Italian herbs & 1.05 & 0.82 & 6.82 \\
\hline 103 & Cocktail nuts & "Borrelnoten" Sateh curry & 1.58 & 2.22 & 12.34 \\
\hline 104 & Cocktail nuts & "Borrelnoten" Spicy & 0.55 & 1.13 & 8.06 \\
\hline 105 & Potato crisps & Kettle & 0.23 & 0.27 & 1.63 \\
\hline 106 & Corn crisps & Vitasia Tikka M asala & 0.29 & 0.11 & 2.79 \\
\hline 107 & "Kroket" & Beef filling, deep fried 7,5 minutes & 0.41 & 0.44 & 4.06 \\
\hline 108 & Sausage roll & Oven heated, 8 minutes & 1.01 & 0.45 & 5.26 \\
\hline 109 & Satay & $\begin{array}{l}\text { Pork, with peanut sauce, microwave } \\
\text { heated } 60 \text { seconds }\end{array}$ & 0.45 & 0.9 & 5.7 \\
\hline 110 & "Frikandel" & Deep fried 7,5 minutes & 0.99 & 1.42 & 5.07 \\
\hline
\end{tabular}

\section{Mixed dishes}

\begin{tabular}{llllll}
\hline & Food & Specification & CML* & CEL* & M G-H1* \\
\hline 111 & Spring roll & $\begin{array}{l}\text { Deep fried, with vegetables and meat } \\
\text { Mozzarella pesto, oven heated }\end{array}$ & 0.2 & 0.07 & 1.53 \\
112 & Pizza & $\begin{array}{l}12 \text { minutes } 175^{\circ} \mathrm{C} \\
\text { With beef, potato, onion, peas, } \\
\text { mayonnaise }\end{array}$ & 0.06 & 0.05 & 0.95 \\
\hline
\end{tabular}

\begin{tabular}{llllll}
\multicolumn{2}{c}{ Soups } & \multicolumn{3}{c}{} & \\
\hline \multicolumn{2}{c}{ Food } & Specification & CML* & CEL* & M G-H1* \\
\hline 114 & $\begin{array}{l}\text { Tomato soup, } \\
\text { canned }\end{array}$ & $\begin{array}{l}\text { Microwave heated, 1,5 minutes } \\
\text { 800 Watt }\end{array}$ & 0 & 0 & 0 \\
115 & Pea soup & Home made, heated & 0.04 & 0.11 & 2.23 \\
\hline
\end{tabular}


Meats, meat products and poultry

\begin{tabular}{|c|c|c|c|c|c|}
\hline & Food & Specification & CML* & CEL* & M G-H1* \\
\hline 116 & $\begin{array}{l}\text { Minced beef ( } 10 \% \\
\text { fat) }\end{array}$ & Beef, fried, 8 minutes & 0.37 & 0.44 & 0.54 \\
\hline 117 & $\begin{array}{l}\text { Red cooked beef } \\
(n=3)^{* *}\end{array}$ & Stewed, 2,5 hours in wine vinegar & $2.03 \pm 0.45$ & $5.63 \pm 1.12$ & $13.48 \pm 6.01$ \\
\hline 118 & Minced beef $(n=2)^{* *}$ & Steamed, $15 \mathrm{~min}$ & $0.44 \pm 0.11$ & $0.25 \pm 0.11$ & $2.06 \pm 1.38$ \\
\hline 119 & M inced beef $(n=2)^{* *}$ & Fried, 10 minutes & $0.51 \pm 0.08$ & $0.31 \pm 0.10$ & $0.86 \pm 0.24$ \\
\hline 120 & Beef steak & Grilled 5 minutes & 0.73 & 0.4 & 0.9 \\
\hline 121 & Roast beef & Oven heated, 1 hour $150 \mathrm{C}$ & 1.01 & 0.41 & 2.09 \\
\hline 122 & Beef steak $(n=3)^{* *}$ & Fried, 4 minutes & $0.12 \pm 0.08$ & $0.23 \pm 0.11$ & $0.34 \pm 0.12$ \\
\hline 123 & $\begin{array}{l}\text { Beef steak (canned) } \\
(n=3)^{* *}\end{array}$ & Pan heated, 10 minutes & $1.07 \pm 0.38$ & $5.63 \pm 2.01$ & $11.96 \pm 2.78$ \\
\hline 124 & $\begin{array}{l}\text { Pork, shoulder } \\
\text { chops }(n=3)^{* *}\end{array}$ & With garlic, fried 12 minutes & $0.15 \pm 0.04$ & $0.35 \pm 0.17$ & $0.51 \pm 0.24$ \\
\hline 125 & Pork $(n=3)^{* *}$ & $\begin{array}{l}\text { Fried, } 3 \text { minutes, then stewed, } \\
40 \text { minutes }\end{array}$ & $0.61 \pm 0.12$ & $1.03 \pm 0.28$ & $1.71 \pm 0.53$ \\
\hline 126 & Pork fillet roulade & Oven heated, $40 \mathrm{~min} 175 \mathrm{C}$ & 0.11 & 0.15 & 0.49 \\
\hline 127 & $\begin{array}{l}\text { M inced beef }(21 \% \\
\text { fat) }\end{array}$ & $50 / 50$ beef and pork, fried 5 minutes & 0.94 & 1.68 & 2.19 \\
\hline 128 & "Slavink" & $\begin{array}{l}\text { M inced beef wrapped in bacon, } \\
\text { fried, } \\
20 \text { minutes }\end{array}$ & 0.14 & 0.09 & 0.77 \\
\hline 129 & Sausage & Fried, 13 minutes & 0.84 & 1.63 & 3.85 \\
\hline 130 & Fried bacon & Fried & 0.72 & 1.82 & 3.01 \\
\hline 131 & Pork & Strips, fried 7 minutes & 1.12 & 1.15 & 1.9 \\
\hline 132 & $\begin{array}{l}\text { Frankfurter, canned } \\
(n=2)^{* *}\end{array}$ & & $4.22 \pm 0.16$ & $1.01 \pm 0.09$ & $4.74 \pm 0.68$ \\
\hline 133 & $\begin{array}{l}\text { Frankfurter, canned } \\
(n=2)^{* *}\end{array}$ & Oven heated, $15 \min 175 \mathrm{C}$ & $3.33 \pm 0.98$ & $1.09 \pm 0.22$ & $4.22 \pm 0.67$ \\
\hline 134 & M eat ball & & 0.83 & 0.9 & 4.61 \\
\hline 135 & M eat ball & Oven heated, $15 \min 175 \mathrm{C}$ & 1.27 & 1.91 & 6.95 \\
\hline 136 & Roasted pork & Roasted & 0.41 & 0.29 & 3.55 \\
\hline 137 & $\begin{array}{l}\text { Pork tenderloin } \\
(n=3)^{* *}\end{array}$ & Fried, 16 minutes & $0.34 \pm 0.25$ & $1.39 \pm 1.14$ & $3.23 \pm 2.27$ \\
\hline 138 & $\begin{array}{l}\text { Gammon steak } \\
(n=3)^{* *}\end{array}$ & Fried, 25 minutes & $0.22 \pm 0.07$ & $0.89 \pm 0.61$ & $1.04 \pm 0.34$ \\
\hline 139 & Schnitzel $(n=3)^{* *}$ & Non-breaded, fried 6 minutes & $0.12 \pm 0.05$ & $0.44 \pm 0.35$ & $0.23 \pm 0.16$ \\
\hline 140 & Rib eye & Fried, 15 minutes & 0.27 & 0.35 & 0.79 \\
\hline 141 & Hamburger & Fried, 8 minutes & 0.2 & 0.29 & 1.58 \\
\hline 142 & Black pudding & $\begin{array}{l}\text { With crushed bacon, fried } \\
16 \text { minutes }\end{array}$ & 4.82 & 7.71 & 63.01 \\
\hline 143 & Lamb, chops $(n=3)^{* *}$ & Fried, 6 minutes & $0.11 \pm 0.03$ & $0.30 \pm 0.08$ & $0.66 \pm 0.22$ \\
\hline 144 & "Rookworst" $(n=2)^{* *}$ & $\begin{array}{l}\text { Smoked sausage, beef, heated in } \\
\text { hot water }\end{array}$ & $0.98 \pm 0.08$ & $0.41 \pm 0.18$ & $1.06 \pm 0.12$ \\
\hline 145 & "Rookworst" ( $n=2)^{* *}$ & $\begin{array}{l}\text { Smoked sausage, pork, heated in } \\
\text { hot water }\end{array}$ & $1.67 \pm 1.27$ & $0.46 \pm 0.15$ & $2.19 \pm 0.27$ \\
\hline 146 & Gammon (boiled) & Cold cut & 0.12 & 0.33 & 0.37 \\
\hline
\end{tabular}


Meats, meat products and poultry

\begin{tabular}{|c|c|c|c|c|c|}
\hline & Food & Specification & CML* & CEL* & M G-H1* \\
\hline 147 & $\begin{array}{l}\text { Ham, shoulder } \\
\text { (boiled) }\end{array}$ & Cold cut & 0.32 & 0.66 & 1.26 \\
\hline 148 & Steak tartare & Sweet onion & 0.08 & 0.04 & 0.21 \\
\hline 149 & Roast beef & Cold cut & 0.28 & 0.41 & 0.44 \\
\hline 150 & Salami & Cold cut & 1.27 & 0.58 & 3.15 \\
\hline 151 & $\begin{array}{l}\text { Salami sausage } \\
\text { saveloy }\end{array}$ & Cold cut & 0.54 & 0.19 & 2.43 \\
\hline 152 & Bacon rashers & Cold cut & 2.54 & 0.93 & 0.71 \\
\hline 153 & Drumsticks & $\begin{array}{l}\text { With skin, oven heated, } 40 \text { minutes, } \\
200^{\circ} \mathrm{C}\end{array}$ & 0.62 & 0.95 & 2.21 \\
\hline 154 & Chicken Wings & With skin, oven heated & 0.82 & 0.78 & 3.04 \\
\hline 155 & $\begin{array}{l}\text { Ragout (chicken } \\
\text { stew) }\end{array}$ & Pan heated & 0.65 & 0.79 & 2.93 \\
\hline 156 & Crusty chicken & Breaded & 0.75 & 0.73 & 4.05 \\
\hline 157 & Chicken fillet & Fried, 30 minutes & 0.13 & 0.27 & 1.41 \\
\hline 158 & Chicken $(n=2)^{*}$ & Boiled, 30 minutes & $0.18 \pm 0.03$ & $0.14 \pm 0.07$ & $0.65 \pm 0.07$ \\
\hline 159 & Chicken $(n=2)^{*}$ & Fried, 10 minutes & $0.34 \pm 0.02$ & $0.26 \pm 0.04$ & $1.16 \pm 0.41$ \\
\hline 160 & Chicken & Microwaved, 10 minutes & 0.92 & 1.02 & 5.16 \\
\hline 161 & Pâté & & 0.09 & 0.07 & 0.29 \\
\hline 162 & Liverwurst $(n=2)^{*}$ & & $0.25 \pm 0.14$ & $0.30 \pm 0.04$ & $1.59 \pm 0.41$ \\
\hline \multicolumn{6}{|c|}{ Bread } \\
\hline & Food & Specification & CML* & CEL* & M G-H1* \\
\hline 163 & Wholemeal bread & & 0.28 & 0.21 & 3.36 \\
\hline 164 & Brown bread & & 0.4 & 0.31 & 6.01 \\
\hline 165 & Rye bread & Dark, brand A & 0.9 & 0.09 & 2.57 \\
\hline 166 & Rye bread & Dark, brand B & 0.58 & 0.18 & 2.74 \\
\hline 167 & Rye bread & Light & 0.59 & 0.18 & 5.22 \\
\hline 168 & White bread $(n=3)^{* *}$ & & $0.24 \pm 0.02$ & $0.11 \pm 0.02$ & $2.55 \pm 0.33$ \\
\hline 169 & White bread $(n=3)^{* *}$ & Belgian made & $0.21 \pm 0.05$ & $0.11 \pm 0.01$ & $2.48 \pm 0.19$ \\
\hline 170 & White bread $(n=3)^{* *}$ & German made & $0.31 \pm 0.01$ & $0.13 \pm 0.01$ & $2.09 \pm 0.15$ \\
\hline 171 & White bread $(n=3)^{* *}$ & Dutch made & $0.13 \pm 0.02$ & $0.07 \pm 0.01$ & $2.18 \pm 0.04$ \\
\hline 172 & Currant bread & & 1.11 & 0.56 & 3.35 \\
\hline 173 & $\begin{array}{l}\text { Luxurious } \\
\text { breads/buns }\end{array}$ & $\begin{array}{l}\text { Baguette, oven heated, } \\
9 \text { minutes, } 200^{\circ} \mathrm{C}\end{array}$ & 0.22 & 0.1 & 2.28 \\
\hline 174 & Croissant & & 1.04 & 1.33 & 9.89 \\
\hline 175 & Rusk & & 1.96 & 1.44 & 23.1 \\
\hline 176 & Knäckebröd & & 0.96 & 1.41 & 13.5 \\
\hline 177 & Toast (bread) & $2 \frac{1}{2}$ minutes in toaster & 0.52 & 0.55 & 4.16 \\
\hline 178 & Toast (cracker) & & 2.31 & 0.76 & 9.97 \\
\hline 179 & Baguette, toasted & $2 \frac{1}{2} 2$ minutes in toaster & 0.23 & 0.1 & 3.61 \\
\hline 180 & $\begin{array}{l}\text { White bread } \\
\text { without crust }\end{array}$ & Belgian made & 0.21 & 0.03 & 1.86 \\
\hline 181 & \multicolumn{2}{|c|}{ Wholemeal bread without crust } & 0.24 & 0.05 & 4.1 \\
\hline 182 & Pita bread & Oven heated, $15 \mathrm{~min}, 175^{\circ} \mathrm{C}$ & 0.25 & 0.06 & 2.82 \\
\hline
\end{tabular}


Pastry and biscuits

\begin{tabular}{|c|c|c|c|c|c|}
\hline & Food & Specification & CML* & CEL* & MG-H1* \\
\hline 183 & $\begin{array}{l}\text { Dutch spiced cake } \\
(n=3)^{* *}\end{array}$ & Dutch made (Batch 1) & $0.79 \pm 0.05$ & $1.22 \pm 0.09$ & $20.31 \pm 0.82$ \\
\hline 184 & $\begin{array}{l}\text { Dutch spiced cake } \\
(n=3)^{* *}\end{array}$ & Dutch made (Batch 2) & $0.91 \pm 0.02$ & $1.13 \pm 0.27$ & $19.76 \pm 1.81$ \\
\hline 185 & $\begin{array}{l}\text { Dutch spiced cake } \\
(n=3)^{* *}\end{array}$ & Belgian made (Batch 1) & $0.65 \pm 0.09$ & $0.84 \pm 0.19$ & $12.97 \pm 2.42$ \\
\hline 186 & $\begin{array}{l}\text { Dutch spiced cake } \\
(n=3)^{* *}\end{array}$ & Belgian made (Batch 2) & $0.74 \pm 0.17$ & $0.84 \pm 0.27$ & $15.87 \pm 2.12$ \\
\hline 187 & $\begin{array}{l}\text { Dutch spiced cake } \\
(n=3)^{* *}\end{array}$ & German made (Batch 1) & $0.79 \pm 0.11$ & $0.91 \pm 0.08$ & $8.07 \pm 0.14$ \\
\hline 188 & $\begin{array}{l}\text { Dutch spiced cake } \\
(n=3)^{* *}\end{array}$ & German made (Batch 2) & $0.91 \pm 0.08$ & $1.24 \pm 0.33$ & $10.87 \pm 0.78$ \\
\hline 189 & Apfelstrudel & Oven heated 40 minutes, $200^{\circ} \mathrm{C}$ & 0.07 & 0.05 & 0.97 \\
\hline 190 & Apricot pie & & 0.25 & 0.47 & 3.47 \\
\hline 191 & Cream pie & & 0.48 & 0.03 & 1.14 \\
\hline 192 & Tompouce & & 0.27 & 0.24 & 2.23 \\
\hline 193 & Pancake & With mascarpone & 0.49 & 0.17 & 1.38 \\
\hline 194 & Cake & & 1.75 & 0.23 & 2.61 \\
\hline 195 & Syrup waffle & "Stroopwafel" & 0.46 & 0.58 & 11.31 \\
\hline 196 & Cake & With almond paste & 0.51 & 0.55 & 5.57 \\
\hline 197 & Biscuit & Multigrain & 1.33 & 1.06 & 19.62 \\
\hline 198 & Biscuit & With peanuts & 2.59 & 3.42 & 36.9 \\
\hline 199 & Biscuit & Speculoos & 2.47 & 1.99 & 28.31 \\
\hline 200 & Biscuit & With currants and cherry & 1.03 & 0.48 & 3.99 \\
\hline 201 & Rice cracker & With cheese powder & 1.13 & 1.04 & 11.34 \\
\hline 202 & Rice cracker & With sea salt & 0.91 & 0.75 & 8.01 \\
\hline
\end{tabular}

Cereals and cereal products

\begin{tabular}{llllll}
\hline & Food & Specification & CML* & CEL* & M G-H1* \\
\hline 203 & Cereals & Honey loops & 1.2 & 0.7 & 3.6 \\
204 & Cereals & Honey granola & 1.04 & 1.02 & 9.44 \\
205 & Cereals & Crunchy muesli & 0.46 & 0.46 & 8.61 \\
206 & Cereals $(n=8)^{* *}$ & Cornflakes & $0.66 \pm 0.08$ & $0.79 \pm 0.13$ & $12.12 \pm 3.53$ \\
207 & Cereals & Puffed wheat & 1.27 & 0.84 & 15.61 \\
208 & Cereals & Frosted flakes & 0.63 & 0.53 & 25.89 \\
209 & Cereals & Rice crispies & 0.59 & 0.47 & 14.7 \\
210 & Cereals & Cooked oats & 1.17 & 0.43 & 11.31 \\
211 & Cereals & Miniwheats & 1.96 & 1.64 & 41.6 \\
212 & Rice, boiled & Basmati & 0.07 & 0.02 & 1.37 \\
213 & Rice, fried & Boiled, then fried & 0.96 & 1.32 & 8.59 \\
214 & Rice, fried & Fried in olive oil & 0.12 & 0.03 & 4.17 \\
215 & Pasta, boiled & Penne & 0.59 & 0.11 & 2.51 \\
216 & Pasta, boiled & Spaghetti & 0.82 & 0.13 & 3.02 \\
217 & Pasta, boiled & Macaroni & 0.43 & 0.06 & 1.41 \\
\hline
\end{tabular}

** Data expressed as mean $\pm s d$ 

Dietary intake of advanced glycation end products is associated with higher levels of advanced glycation endproducts in plasma and urine: the CODAM study

Jean LJ.M. Scheijen, Nordin M.J. Hanssen, Marleen M. van Greevenbroek, Carla J. Van der Kallen, Edith J.M. Feskens, Coen D.A. Stehouwer, Casper G. Schalkwijk 


\section{Abstract}

Background \& Aims: Advanced glycation endproducts (AGEs) are formed by the reaction between reducing sugars and proteins. AGEs in the body have been associated with several age-related diseases. High-heat treated and most processed foods are rich in AGEs. The aim of our study was to investigate whether dietary AGEs, are associated with plasma and urinary AGE levels.

Methods: In 450 participants of the Cohort on Diabetes and Atherosclerosis Maastricht study (CODAM study) we measured plasma and urine concentrations of the AGEs $\mathrm{N}^{\varepsilon}$-(carboxymethyl)lysine (CML), $\mathrm{N}^{\varepsilon}$-(1-carboxyethyl)lysine (CEL) and $\mathrm{N}^{\delta}$-(5-hydro-5methyl-4-imidazolon-2-yl)-ornithine (MG-H1) using UPLC-MS/MS. We also estimated dietary intake of CML, CEL and MG-H1 with the use of a dietary AGE database and a food frequency questionnaire (FFQ). We used linear regression to investigate the association between standardized dietary AGE intake and standardized plasma or urinary AGE levels, after adjustment for age, sex, glucose metabolism status, waist circumference, kidney function, energy- and macronutrient intake, smoking status, physical activity, alcohol intake, LDL-cholesterol and markers of oxidative stress.

Results: We found that higher intake of dietary CML, CEL and M G-H1 was associated with significantly higher levels of free plasma and urinary CML, CEL and M G-H1 ( $\beta C M L=0.253$ (95\% Cl 0.086; 0.415), $\beta C E L=0.194(95 \% \mathrm{Cl} 0.040 ; 0.339), \beta M \mathrm{G}-\mathrm{H} 1=0.223(95 \% \mathrm{Cl} 0.069$; 0.373 ) for plasma and $\beta C M L=0.223(95 \% \mathrm{Cl} 0.049 ; 0.393), \beta C E L=0.180(95 \% \mathrm{Cl} 0.019$; $0.332), \beta M G-H 1=0.196(95 \% \mathrm{Cl} 0.037 ; 0.349)$ for urine, respectively). In addition, we observed non-significant associations of dietary AGEs with their corresponding protein bound plasma AGEs.

Conclusion: We demonstrate that higher intake of dietary AGEs is associated with higher levels of AGEs in plasma and urine. Our findings may have important implications for those who ingest a diet rich in AGEs. 


\section{Introduction}

Advanced glycation endproducts (AGEs) are a large and heterogeneous family of sugarmodified proteins, which have been linked to a plethora of age-related diseases including diabetes, atherosclerosis, chronic obstructive pulmonary disease and Alzheimer's disease ${ }^{1-3}$.

In 1912, the French chemist Louis Camile Maillard discovered the reaction between reducing sugars and free amino acids on proteins ${ }^{4}$, leading to the formation of AGEs. At body temperature, the Maillard reaction takes place at a very slow pace. In contrast, in foods which had a prolonged exposure to high heat AGEs were formed rapidly ${ }^{5}$. The consumption of processed foods that were cooked at high temperatures has increased over the past decades ${ }^{6}$. As a consequence, the exposure to dietary AGEs have also increased and may be a risk factor for chronic diseases ${ }^{7}$. Indeed, recent studies have shown associations of dietary AGEs with insulin sensitivity ${ }^{8}$, abdominal obesity and hypertriglyceridemia ${ }^{9}$ and with poorer memory in Alzheimer's disease ${ }^{10}$. AGEs in the body may contribute to development of age-related diseases through several mechanisms, such as interaction with the receptor for AGEs (RAGE) $)^{11}$ and crosslinking on long-lived proteins $^{12}$. However, whether dietary AGEs are substantially absorbed in the digestive tract and released into the circulation to contribute to any of these effects remains unclear.

The studies so far addressing whether high intake of dietary AGEs lead to increased plasma and urinary AGEs have not been conclusive ${ }^{13-22}$, because most of these studies have been small and have not taken into account confounding factors such as kidney function or dietary energy intake, which may explain the associations ${ }^{19,20}$. Moreover, different analytical techniques to analyze AGE content in food and plasma samples were used in these studies, which may have led to differences in AGE concentrations ${ }^{21,22}$.

Taken these considerations into account, the aim of the current study was to establish whether higher intake of dietary AGEs are associated with higher levels of plasma and urinary AGEs. In a previous study, we developed a new dietary AGE database of three major AGEs $\mathrm{N}^{\varepsilon}$-(carboxymethyl)lysine (CML), $\mathrm{N}^{\varepsilon}$-(1-carboxyethyl)lysine (CEL) and $\mathrm{N}^{\delta}$-(5-hydro-5-methyl-4-imidazolon-2-yl)-ornithine (MG-H1), as analyzed in 190 specific food items, based on a state-of-the art ultra-performance liquid chromatography tandem mass spectrometry (UPLC-MS/MS) technique ${ }^{23}$. In the present study, we linked information on AGE-content of these food items to a food frequency questionnaire (FFQ) and calculated the consumption of AGEs in a cohort of 450 participants. We subsequently examined the association of dietary AGE intake with three major plasma and urinary AGEs (CML CEL and M G-H1), adjusting for major potential confounders. 


\section{Materials and methods}

\section{Study population}

Cross-sectional analyses were performed on data from the Cohort on Diabetes and Atherosclerosis Maastricht study (CODAM), which includes 574 individuals with an elevated risk for T2DM and cardiovascular disease and described in detail elsewhere ${ }^{24}$. Participants were instructed to withhold their lipid-lowering medication for a fortnight prior the first visit, and not to consume any alcoholic drinks, coffee and/or tea, not to smoke, and withhold all other medication the day before. The habitual dietary intake over the last twelve months of all participants was established by a self-administered food frequency questionnaire (FFQ) which queried 194 foods $^{25}$.

Individuals were excluded if they did not qualify to report the FFQ successfully ( $n=56$, i.e. more than $10 \%$ items missing on the FFQ). Participants who reported an energy intake outside the range of 800-4200 kcal/day for men and 600-3500 kcal/day for women were also excluded $(n=6)$. Due to sample availability, 450 participants were used for statistical analysis (figure 9.1).

Fasting and 2-h postload glucose concentrations were used to classify the study participants' glucose metabolism status (GMS), described in details elsewhere ${ }^{26}$. Questionnaires were used to assess smoking behavior (never, ever, or current smoker) and use of medication (lipid-, glucose-, and blood pressure-lowering medication). Plasma creatinine levels were measured with the Jaffe diagnostic test (Roche Diagnostics, Mannheim, Germany), and the estimated glomerular filtration rate (eGFR) was calculated using the Chronic Kidney Disease Epidemiology Collaboration (CKD-EPI) equation ${ }^{27}$. Plasma malondialdehyde (MDA) levels were measured with a reagent kit for highperformance liquid chromatography analyses (Chromsystems Instruments and Chemicals, Munich, Germany), and total antioxidative status (TAS) was measured in serum with an enzymatic kit (Randox Diagnostics, County Antrim, U.K.). LDL cholesterol was calculated with the Friedewald formula ${ }^{28}$, after measurement of fasting total cholesterol, HDLcholesterol and triglyceride levels.

All subjects gave written informed consent. The study was approved by the local Medical Ethical Committee of the University of M aastricht and University Hospital M aastricht.

\section{Food, plasma and urinary AGE quantification}

Protein-bound AGEs in individual food items and in plasma, and free AGEs in plasma and in urine were analyzed as described in detail elsewhere ${ }^{23,29}$. In short, foods were obtained from local supermarkets or restaurants and prepared according to the instructions on the label. For UPLC-MS/MS analysis a representative sample of each food item, equivalent to $5 \mathrm{mg}$ of protein, was used. For protein-bound and free plasma AGEs, $25 \mu \mathrm{L}$ and $50 \mu \mathrm{L}$ plasma was used, respectively. 
Forty microliters were used for free urinary AGE analysis. All samples were derivatized with butanolic hydrochloric acid and subsequently detected in ESI positive multiple reaction monitoring (MRM) mode using a Xevo TQ MS (Waters, Milford, USA). Quantification of $\mathrm{CML}$, CEL and M G-H1 was performed by calculating the peak area ratio of each unlabeled peak area to the corresponding internal standard peak area. In plasma, the intra- and inter-assay variation of protein-bound CML and CEL were between 4.8 and 9.7\% and for free CML, CEL and M G-H1 between 2.8 and $7.1 \%$. In urine, the intra- and inter-assay variation of free CML, CEL and MG-H1 were between 3.7 and $6.6 \%$. In food matrix, the intra- and inter-assay variation of protein-bound $\mathrm{CM}$ L, CEL and M G-H1 were between 1.7 and $29.1 \%$.

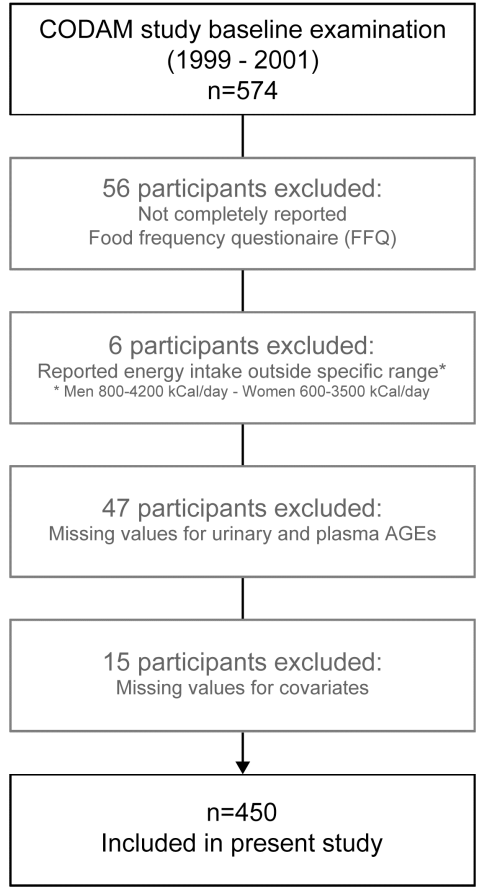

Figure 9.1 Flowchart for the exclusion of participants in the current CODAM study.

\section{Assessment of AGE intake}

For UPLC-MS/MS protein-bound AGE analysis, food items were selected based on the items included in the FFQ of CODAM, which queried 194 foods commonly consumed in the Netherlands. Each food item in the FFQ was quantified as grams of food per day and analyzed for AGE content. The AGE intake was estimated for each participant by multiplying the food intake in $\mathrm{g} /$ day with the concentration of AGEs in $\mathrm{mg} / \mathrm{g}$ in each representative individual food item. 
To calculate the total AGE intake per participant, the estimated AGE levels of the various food items were added together. Some of the food items had unspecific descriptions in the FFQ (e.g. "sweet spreads"). These items were further defined as an average of this food group (e.g. for "sweet spreads" the average AGE content of apple syrup, chocolate spread, chocolate sprinkles, fruit sprinkles and jam was used). Moreover, some of the food items in the FFQ were not analyzed for AGEs. These food items were matched with food items based on ingredient profile. To this end, estimation was made for the AGE content of these missing food items.

\section{Statistical analyses}

All statistical analyses were carried out using the IBM SPSS Statistics Software, version 23.0 (IBM Corporation, Armonk, NY, USA). General characteristics of the CODAM population were compared across tertiles of the dietary AGE score with the use of ANOVA or $\mathrm{X}^{2}$-tests, for continuous or categorical data, respectively. We $\log _{\mathrm{e}}$-normalised all plasma and urinary AGEs to reduce the potential influence of outliers and to obtain a normal distribution, needed for calculation of Z-scores. An AGE score was calculated that represents an individual's overall intake of AGEs via their habitual diet. For this, first the Z-scores were calculated for each dietary AGE (CM L, CEL and M G-H1) as follows: for each individual dietary AGE, a Z-score was calculated according to the formula: [(individual dietary AGE - population mean dietary AGE)/population standard deviation dietary AGE]. The resulting Z-scores, of each dietary AGE (CML, CEL and MG-H1), were then averaged into an overall dietary AGE Z-score and this overall dietary AGE Z-score was subsequently standardized.

We used linear regression analyses to investigate the association between dietary and plasma or urinary AGE levels, after adjustments for participant characteristics (age, sex, glucose metabolism status and waist circumference: model 1) and additionally for kidney function (estimated glomerular filtration rate (eGFR): model 2), and further for potential dietary and lifestyle confounding factors (energy intake and protein-, saturated fat-, carbohydrate- and fibre-intake, all macronutrients expressed as energy percentage and smoking, physical activity and alcohol intake: model 3), and for potential confounding by lipid metabolism and oxidative stress (LDL-cholesterol, malondialdehyde (MDA) and total antioxidant status (TAS): model 4). Participants with missing values ( $n=14$ ) on co-variates were excluded listwise. We also tested whether associations differed for sex (sex x dietary AGE), eGFR (eGFR $x$ dietary AGE) and the presence of impaired glucose metabolism or diabetes (presence of impaired glucose metabolism or diabetes $x$ dietary AGE), by adding interaction terms to our models ( $p$-values for interaction were $\varangle 0.1$ ). Overall, we found no consistent interactions (data not shown). 


\section{Results}

Table 9.1 shows the general characteristics of the total population of the CODAM study as stratified according to tertiles of dietary AGEs. Although we observed important quantitative differences in dietary AGE levels, with MG-H1 being most abundant, we observed strong correlations between the AGE intake (CM L vs. CEL $r_{\text {pearson: }}$ 0.811, CM L vs. MG-H1 $r_{\text {pearson: }} 0.795$ and CEL vs. MG-H1 $r_{\text {pearson: }}$ 0.880; for all $p<0.001$ ). Plasma free-, plasma protein-bound- and urinary AGEs did not differ between the tertiles of dietary AGEs (table 9.1). M ale participants had higher intake of dietary AGEs ( $p \varangle 0.001$, table 9.1). In addition, HDL cholesterol, protein intake and fibre intake were lower in the individuals with higher intake of dietary AGEs ( $p \varangle 0.05$, table 9.1) and saturated fat-, energy- and alcohol intake were higher in the individuals with higher intake of dietary AGEs $(p \varangle 0.05$, table 9.1).

\section{Associations between dietary AGE intake and protein-bound plasma AGES}

After adjustment for age, sex, glucose metabolism status and waist circumference, we found no significant association between dietary $C M L$ and protein-bound plasma $C M L$ levels (table 9.2, model 1). Further adjustment for eGFR did not change these results (table 9.2, model 2). In the fully adjusted model we observed a weak positive association of dietary CML and protein-bound plasma CML, but this was not significant (table 9.2, model 4). We observed similar findings for dietary CEL and protein-bound CEL (table 9.2).

\section{Associations between dietary AGE intake and free plasma AGES}

After adjustment for age, sex, glucose metabolism status and waist circumference we observed positive associations between dietary intake of CML, CEL and MG-H1 and their free levels in plasma (table 9.3, model 1). Further adjustment for eGFR (table 9.3, model 2), energy- and macronutrient intake, lifestyle factors, lipid metabolism and markers of oxidative stress (table 9.3, model 3 and 4) did not change the results overall. In the fully adjusted model, these associations were approximately twice as strong for $\mathrm{CM} \mathrm{L,} \mathrm{CEL} \mathrm{and}$ MG-H1 (table 9.3, model 4 vs. model 1).

\section{Associations between dietary AGE intake and free urinary AGES}

In line with the findings for free plasma AGEs, higher dietary intake of $C M L, C E L$ and $M G$ $\mathrm{H} 1$ were associated with higher urinary $\mathrm{CM} \mathrm{L}, \mathrm{CEL}$ and M G-H1 levels (table 9.4, model 1-4). Adjustment for eGFR did not change the results (table 9.4, model 2). 


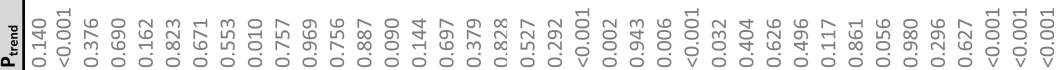

商

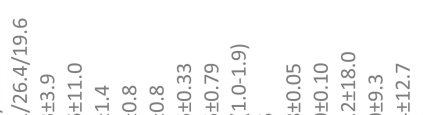

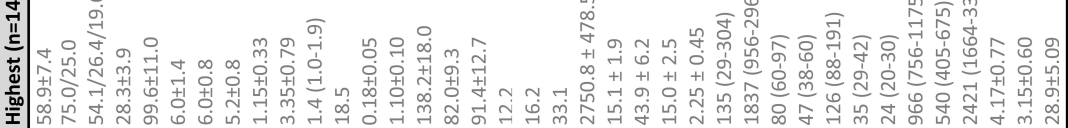

岁

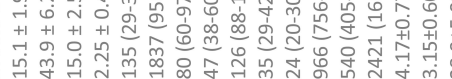

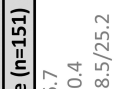

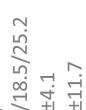

in @

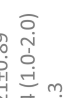

กㅇำ

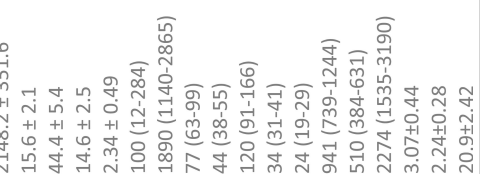

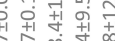

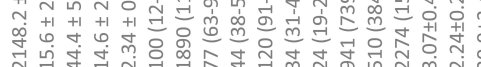

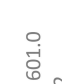

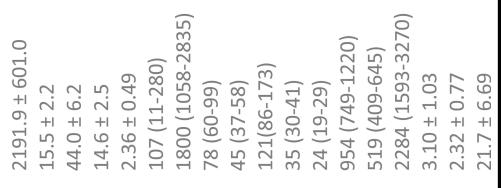

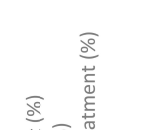

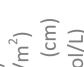

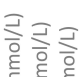

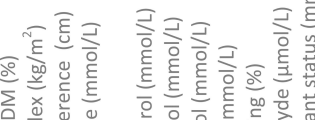

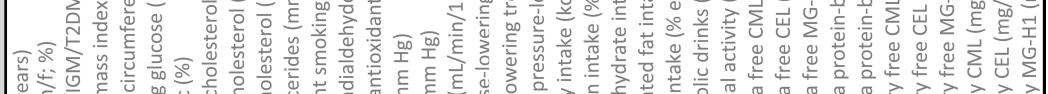

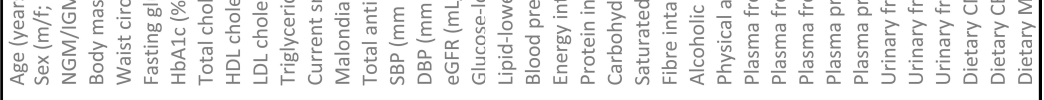


Table 9.2 Associations between dietary AGEs and protein-bound plasma AGEs.

\begin{tabular}{lllll}
\hline \multicolumn{5}{l}{ Protein-bound plasma CML } \\
\\
Model & $\beta$ & $95 \% \mathrm{Cl}$ & $\mathbf{p}$ \\
\hline Dietary CML & Crude & 0.039 & $-0.054 ; 0.128$ & 0.42 \\
& 1 & 0.048 & $-0.041 ; 0.133$ & 0.30 \\
& 2 & 0.046 & $-0.042 ; 0.131$ & 0.32 \\
& 3 & 0.122 & $-0.042 ; 0.277$ & 0.15 \\
& 0.130 & $-0.032 ; 0.283$ & 0.12 \\
\hline
\end{tabular}

\begin{tabular}{|c|c|c|c|c|}
\hline & \multicolumn{4}{|c|}{ Protein-bound plasma CEL } \\
\hline & Model & $\beta$ & $95 \% \mathrm{Cl}$ & $p$ \\
\hline \multirow[t]{5}{*}{ Dietary CEL } & Crude & 0.112 & $0.017 ; 0.199$ & 0.02 \\
\hline & 1 & 0.105 & $0.005 ; 0.197$ & 0.04 \\
\hline & 2 & 0.101 & $0.002 ; 0.194$ & 0.05 \\
\hline & 3 & 0.136 & $-0.030 ; 0.293$ & 0.11 \\
\hline & 4 & 0.112 & $-0.050 ; 0.268$ & 0.18 \\
\hline
\end{tabular}

Table 9.3 Associations between dietary AGEs and free plasma AGEs.

\begin{tabular}{|c|c|c|c|c|}
\hline & \multicolumn{4}{|c|}{ Free plasma CML } \\
\hline & Model & $\beta$ & $95 \% \mathrm{Cl}$ & $p$ \\
\hline \multirow[t]{7}{*}{ Dietary CML } & Crude & 0.055 & $-0.039 ; 0.147$ & 0.26 \\
\hline & 1 & 0.099 & $0.006 ; 0.190$ & 0.04 \\
\hline & 2 & 0.094 & $0.004 ; 0.183$ & 0.04 \\
\hline & 3 & 0.224 & $0.057 ; 0.386$ & 0.01 \\
\hline & 4 & 0.253 & $0.086 ; 0.415$ & $\varangle 0.01$ \\
\hline & \multicolumn{4}{|c|}{ Free plasma CEL } \\
\hline & Model & $\beta$ & $95 \% \mathrm{Cl}$ & $p$ \\
\hline \multirow[t]{7}{*}{ Dietary CEL } & Crude & 0.086 & $-0.008 ; 0.176$ & 0.07 \\
\hline & 1 & 0.098 & $0.003 ; 0.188$ & 0.04 \\
\hline & 2 & 0.086 & $-0.007 ; 0.174$ & 0.07 \\
\hline & 3 & 0.208 & $0.053 ; 0.353$ & 0.01 \\
\hline & 4 & 0.194 & $0.040 ; 0.339$ & 0.01 \\
\hline & \multicolumn{4}{|c|}{ Free plasma MG-H1 } \\
\hline & Model & $\beta$ & $95 \% \mathrm{Cl}$ & $p$ \\
\hline \multirow[t]{5}{*}{ Dietary M G-H1 } & Crude & 0.127 & $0.033 ; 0.219$ & 0.01 \\
\hline & 1 & 0.150 & $0.056 ; 0.241$ & $\varangle 0.01$ \\
\hline & 2 & 0.135 & $0.043 ; 0.224$ & $\varangle 0.01$ \\
\hline & 3 & 0.239 & $0.085 ; 0.390$ & $\varangle 0.01$ \\
\hline & 4 & 0.223 & $0.069 ; 0.373$ & $\varangle 0.01$ \\
\hline
\end{tabular}


Table 9.4 Associations between dietary AGEs and free urinary AGEs.

\begin{tabular}{lllll}
\hline & \multicolumn{2}{l}{ Free urine CML } & \\
& Model & $\beta$ & $95 \% \mathrm{Cl}$ & $\mathbf{p}$ \\
\hline Dietary CML & Crude & 0.045 & $-0.049 ; 0.138$ & 0.35 \\
& 1 & 0.127 & $0.033 ; 0.218$ & 0.01 \\
& 2 & 0.127 & $0.033 ; 0.218$ & 0.01 \\
& 3 & 0.211 & $0.038 ; 0.380$ & 0.02 \\
& 4 & 0.223 & $0.049 ; 0.393$ & 0.01 \\
\hline
\end{tabular}

\begin{tabular}{lllll}
\hline & \multicolumn{2}{l}{ Free urine CEL } & & \\
& Model & $\beta$ & $95 \% \mathrm{Cl}$ & $\boldsymbol{p}$ \\
\hline Dietary CEL & Crude & 0.056 & $-0.037 ; 0.146$ & 0.25 \\
& 1 & 0.121 & $0.024 ; 0.211$ & 0.01 \\
& 2 & 0.124 & $0.027 ; 0.214$ & 0.01 \\
& 3 & 0.189 & $0.027 ; 0.340$ & 0.02 \\
& 4 & 0.180 & $0.019 ; 0.332$ & 0.03 \\
\hline
\end{tabular}

\begin{tabular}{lllll}
\hline & \multicolumn{2}{l}{ Free urine MG-H1 } & & \\
& Model & $\beta$ & $95 \% \mathrm{Cl}$ & P \\
\hline Dietary M G-H1 & Crude & 0.066 & $-0.028 ; 0.157$ & 0.17 \\
& 1 & 0.134 & $0.039 ; 0.224$ & $\varangle 0.01$ \\
& 2 & 0.134 & $0.043 ; 0.228$ & $\varangle 0.01$ \\
& 3 & 0.205 & $0.045 ; 0.357$ & 0.01 \\
& 4 & 0.196 & $0.037 ; 0.349$ & 0.02 \\
\hline
\end{tabular}

Table 9.2, 9.3 and 9.4:

Data were analyzed using linear regression analyses. $\beta$ S are expressed as 1 SD increase of urinary/plasma AGE per 1SD increase of dietary AGE. All plasma and urinary AGEs were Ln-normalised in all analyses.

Model 1: adjusted for age, sex, glucose metabolism status, waist circumference

Model 2: model $1+$ eGFR

Model 3: model 2 + total energy-, protein-, saturated fat-, carbohydrate- and fibre-intake, smoking, physical activity, alcohol intake

Model 4: model $3+$ LDL cholesterol, malondialdehyde, total antioxidant status

\section{Discussion}

This study is, to our knowledge, the first to demonstrate that higher dietary intake of $\mathrm{CML}$, CEL and M G-H1, as estimated with a FFQ, is associated significantly with higher free plasma and urinary levels of AGES, as measured with UPLC-MS/MS. We found a weak non-significant association of dietary AGEs with their corresponding protein bound plasma AGEs. In the current study we estimated dietary CM L, CEL and M G-H1. Since these compounds were highly correlated, despite quantitative differences, we assume that these three AGEs were well-representative for dietary AGE contents. 
Studies so far have suggested that dietary AGEs are associated with circulating $A_{G E s}{ }^{13,15-18,30}$. Our current study expands on these findings in several important ways. First, we have used a state-of-the-art UPLC-MS/MS technique to analyze three different dietary AGEs and circulating AGEs. Moreover, we performed UPLC-M S/MS plasma and urinary analyses in a large and well-defined cohort study to investigate the associations between three different dietary AGEs and plasma and urinary AGEs. Another major strength of our study is that we were able to use a database of AGEs, as measured with UPLC-M S/M S $^{23}$, in 190 food items to calculate the habitual intake of dietary AGEs based on a FFQ. However, one important limitation of our study was that our FFQ did not include detailed information about food preparation techniques on all food items. We and others have shown that differences in these cooking techniques and duration of heating are fundamental in the formation of AGEs in food ${ }^{5,23,31}$. However, approximately $90 \%$ of the FFQ food items were either uncooked, such as fruit, raw vegetables and drinks or were pre-processed foods which were produced by different manufacturers, such as cookies, bread or peanut butter. Our FFQ consisted of only $10 \%$ of food items which have to be prepared by the participant itself, such as meat, fish, potatoes or pasta. We have included detailed information on the preparation of potatoes in the FFQ, such as boiling, frying or baking. Unfortunately, no detailed information of the preparation, in particular duration of heating, of meat or fish was available. However, our dAGE database ${ }^{23}$, which was used to estimate AGE-intake as calculated with FFQ data, consisted of meat- and fish products which were prepared according to the instructions of the manufacturer's label or using the most common preparation technique. Perhaps this will have led to a source of variance in estimating the dietary AGE content, but this was only true for a minority of the food items. Nonetheless, we still found clear and consistent associations between estimated dietary AGE intake and plasma AGE levels.

Our crude analysis only revealed an association between higher dietary AGEs and higher plasma and urinary AGE levels. Particularly after adjustment for sex, age, glucose metabolism and waist (model 1), and further adjustment for the major macronutrients and lifestyle factors (model 3) even stronger associations were found between dietary AGEs and free plasma and urinary AGEs. The strong effect of confounding factors on the reported associations may at least partly explain conflicting findings between previous studies which have not taken into account these confounding factors ${ }^{19,20}$.

Dietary AGE-modified proteins are mainly digested into free glycated and non-glycated amino acids and small peptides ${ }^{32}$ and thus protein-bound dietary AGEs are expected to enter the circulation predominately in the free form. Subsequently, these AGEs are excreted in the urine ${ }^{16}$. We found no association of dietary AGEs with protein-bound AGEs in plasma, suggesting that the vast majority of the protein-bound AGEs in the human body are formed by the non-enzymatic reaction of glucose, $\alpha$-oxoaldehydes and other saccharide derivatives with proteins, nucleotides and lipids. On the contrary, we found an association of dietary AGEs with free AGEs in plasma. This is in accordance with a recent study which has showed that free plasma AGEs were mainly released from the protein-bound dietary AGEs and hereby enter the circulation predominantly ${ }^{32}$. In line, we found that associations between dietary AGEs and free plasma AGEs are stronger than 
with protein-bound plasma AGEs. Two intervention studies were in line with these results $^{13,16}$. Birlouez-Aragon et al, reported a significant increase of $7 \%$ protein-bound plasma CML after administering a high AGE diet versus a significant increase of $40 \%$ free urinary $\mathrm{CML}^{16}$. Furthermore, the associations between the estimated dietary AGE intake with free plasma AGEs is in line with animal intervention studies ${ }^{33,34}$. In these studies, low- and high-AGE diets were tested in animals and significantly increased levels of free plasma $C M L$, but not protein-bound plasma $C M L$, were found ${ }^{33,34}$. In the same study, increased plasma levels of SRAGE and a pro-inflammatory response was reported after administering a high-AGE diet ${ }^{33}$ which implicates important biological consequences.

In humans, the beneficial effects of a low AGE-diet have been described ${ }^{8,13,35}$. If a highAGE diet in humans, and contemporaneously increased levels of free plasma AGEs, can lead to an increased risk for development of chronic diseases is still under investigation. In our observational study we investigated the association of a habitual diet with similar results.

In summary, we have shown that higher levels of dietary AGEs were significantly associated with higher levels of free plasma and urinary AGEs. These findings are an important step in understanding the metabolic transit of these dietary AGEs. Several studies indicate that dietary AGEs are associated with poor health ${ }^{8-10,16,20}$. Future prospective studies should address whether dietary AGEs are associated with adverse outcomes such as accelerated development of cardiovascular disease, diabetes and other age-related diseases. This line of investigation may eventually lead to new dietary recommendations for both the general public and perhaps specific patients groups in particular. 


\section{References}

1 van Eupen, M. G. et al. Plasma levels of advanced glycation endproducts are associated with type 1 diabetes and coronary artery calcification. Cardiovasc Diabetol 12, 149, doi:10.1186/1475-2840-12149 (2013).

Hanssen, N. M. et al. Higher levels of advanced glycation endproducts in human carotid atherosclerotic plaques are associated with a rupture-prone phenotype. Eur Heart J 35, 1137-1146, doi:10.1093/eurheartj/eht402 (2014).

3 Gopal, P. et al. Plasma advanced glycation end-products and skin autofluorescence are increased in COPD. Eur Respir J 43, 430-438, doi:10.1183/09031936.00135312 (2014).

Maillard, L. C. Action des acides aminés sur les sucres: formation des mélanoidines par voie méthodique. C.R. Acad. Sci. 154, 66-68 (1912).

Poulsen, M. W. et al. Advanced glycation endproducts in food and their effects on health. Food Chem Toxicol 60, 10-37, doi:10.1016/j.fct.2013.06.052 (2013).

Cordain, L. et al. Origins and evolution of the Western diet: health implications for the 21st century. Am J Clin Nutr 81, 341-354 (2005).

Clarke, R. E., Dordevic, A. L., Tan, S. M., Ryan, L. \& Coughlan, M. T. Dietary Advanced Glycation End Products and Risk Factors for Chronic Disease: A Systematic Review of Randomised Controlled Trials. Nutrients 8, doi:10.3390/nu8030125 (2016).

de Courten, B. et al. Diet low in advanced glycation end products increases insulin sensitivity in healthy overweight individuals: a double-blind, randomized, crossover trial. Am J Clin Nutr, doi:10.3945/ajcn.115.125427 (2016).

Angoorani, P., Ejtahed, H. S., M irmiran, P., M irzaei, S. \& Azizi, F. Dietary consumption of advanced glycation end products and risk of metabolic syndrome. International journal of food sciences and nutrition 67, 170-176, doi:10.3109/09637486.2015.1137889 (2016).

Lubitz, I. et al. High dietary advanced glycation end products are associated with poorer spatial learning and accelerated Abeta deposition in an Alzheimer mouse model. Aging cell 15, 309-316, doi:10.1111/acel.12436 (2016).

11 Gaens, K. H., Stehouwer, C. D. \& Schalkwijk, C. G. Advanced glycation endproducts and its receptor for advanced glycation endproducts in obesity. Curr Opin Lipidol 24, 4-11, doi:10.1097/M OL.0b013e32835aea13 (2013).

Verzijl, N. et al. Crosslinking by advanced glycation end products increases the stiffness of the collagen network in human articular cartilage: a possible mechanism through which age is a risk factor for osteoarthritis. Arthritis and rheumatism 46, 114-123, doi:10.1002/15290131(200201)46:1<114::aid-art10025>3.0.c0;2-p (2002).

Mark, A. B. et al. Consumption of a Diet Low in Advanced Glycation Endproducts for 4 weeks Improves Insulin Sensitivity in Overweight Women. Diabetes Care, doi:10.2337/dc13-0842 (2013).

Poulsen, M. W. et al. Effect of dietary advanced glycation end products on postprandial appetite, inflammation, and endothelial activation in healthy overweight individuals. European journal of nutrition 53, 661-672, doi:10.1007/s00394-013-0574-y (2014). 
Klenovics, K. S. et al. Advanced glycation end products in infant formulas do not contribute to insulin resistance associated with their consumption. PLoS One 8, e53056, doi:10.1371/journal.pone.0053056 (2013).

Birlouez-Aragon, I. et al. A diet based on high-heat-treated foods promotes risk factors for diabetes mellitus and cardiovascular diseases. Am J Clin Nutr 91, 1220-1226, doi:10.3945/ajcn.2009.28737 (2010).

Uribarri, J. et al. Single oral challenge by advanced glycation end products acutely impairs endothelial function in diabetic and nondiabetic subjects. Diabetes Care 30, 2579-2582, doi:10.2337/dc07-0320 (2007).

Macias-Cervantes, M. H. et al. Effect of an advanced glycation end product-restricted diet and exercise on metabolic parameters in adult overweight men. Nutrition 31, 446-451, doi:10.1016/j.nut.2014.10.004 (2015).

Chao, P. C., Huang, C. N., Hsu, C. C., Yin, M. C. \& Guo, Y. R. Association of dietary AGEs with circulating AGEs, glycated LDL, IL-1alpha and MCP-1 levels in type 2 diabetic patients. European journal of nutrition 49, 429-434, doi:10.1007/s00394-010-0101-3 (2010).

Negrean, M. et al. Effects of low- and high-advanced glycation endproduct meals on macro- and microvascular endothelial function and oxidative stress in patients with type 2 diabetes mellitus. Am J Clin Nutr 85, 1236-1243 (2007).

Charissou, A., Ait-Ameur, L. \& Birlouez-Aragon, I. Evaluation of a gas chromatography/mass spectrometry method for the quantification of carboxymethyllysine in food samples. J Chromatogr A 1140, 189-194, doi:10.1016/j.chroma.2006.11.066 (2007).

Tareke, E., Forslund, A., Lindh, C. H., Fahlgren, C. \& Ostman, E. Isotope dilution ESI-LC-M S/MS for quantification of free and total Nepsilon-(1-Carboxymethyl)-L-Lysine and free Nepsilon-(1Carboxyethyl)-L-Lysine: comparison of total Nepsilon-(1-Carboxymethyl)-L-Lysine levels measured with new method to ELISA assay in gruel samples. Food Chem 141, 4253-4259, doi:10.1016/j.foodchem.2013.07.003 (2013).

23 Scheijen, J. L. et al. Analysis of advanced glycation endproducts in selected food items by ultraperformance liquid chromatography tandem mass spectrometry: Presentation of a dietary AGE database. Food Chem 190, 1145-1150, doi:10.1016/j.foodchem.2015.06.049 (2016).

Jacobs, M. et al. The association between the metabolic syndrome and alanine amino transferase is mediated by insulin resistance via related metabolic intermediates (the Cohort on Diabetes and Atherosclerosis Maastricht [CODAM] study). Metabolism 60, 969-975, doi:10.1016/j.metabol.2010.09.006 (2011).

Ocke, M. C. et al. The Dutch EPIC food frequency questionnaire. II. Relative validity and reproducibility for nutrients. Int J Epidemiol 26 Suppl 1, S49-58 (1997).

Maessen, D. E. et al. Post-Glucose Load Plasma alpha-Dicarbonyl Concentrations Are Increased in Individuals With Impaired Glucose Metabolism and Type 2 Diabetes: The CODAM Study. Diabetes Care 38, 913-920, doi:10.2337/dc14-2605 (2015).

27 Levey, A. S. et al. A more accurate method to estimate glomerular filtration rate from serum creatinine: a new prediction equation. Modification of Diet in Renal Disease Study Group. Annals of internal medicine 130, 461-470 (1999). 
28

Friedewald, W. T., Levy, R. I. \& Fredrickson, D. S. Estimation of the concentration of low-density lipoprotein cholesterol in plasma, without use of the preparative ultracentrifuge. Clin Chem 18, 499502 (1972).

29

Hanssen, N. M. et al. Plasma levels of advanced glycation endproducts Nepsilon(carboxymethyl)lysine, Nepsilon-(carboxyethyl)|lysine, and pentosidine are not independently associated with cardiovascular disease in individuals with or without type 2 diabetes: the Hoorn and CODAM studies. J Clin Endocrinol M etab 98, E1369-1373, doi:10.1210/jc.2013-1068 (2013).

Sebekova, $\mathrm{K}$. et al. Plasma concentration and urinary excretion of $\mathrm{N}$ epsilon-(carboxymethyl)/ysine in breast milk- and formula-fed infants. Ann N Y Acad Sci 1126, 177-180, doi:10.1196/annals.1433.049 (2008).

Uribarri, J. et al. Advanced glycation end products in foods and a practical guide to their reduction in the diet. Journal of the American Dietetic Association 110, $911-916$ e912, doi:10.1016/j.jada.2010.03.018 (2010).

32 Hellwig, M., Matthes, R., Peto, A., Lobner, J. \& Henle, T. N-epsilon-fructosyllysine and N-epsiloncarboxymethyllysine, but not lysinoalanine, are available for absorption after simulated gastrointestinal digestion. Amino acids 46, 289-299, doi:10.1007/s00726-013-1501-5 (2014).

Elmhiri, G. et al. Formula-derived advanced glycation end products are involved in the development of long-term inflammation and oxidative stress in kidney of IUGR piglets. Molecular nutrition \& food research 59, 939-947, doi:10.1002/mnfr.201400722 (2015). Roncero-Ramos, I. et al. M etabolic transit of N(epsilon)-carboxymethyl-lysine after consumption of AGEs from bread crust. Food \& function 4, 1032-1039, doi:10.1039/ c3fo30351a (2013).

5 Vlassara, $\mathrm{H}$. et al. Oral AGE restriction ameliorates insulin resistance in obese individuals with the metabolic syndrome: a randomised controlled trial. Diabetologia 59, 2181-2192, doi:10.1007/s00125-016-4053-x (2016). 


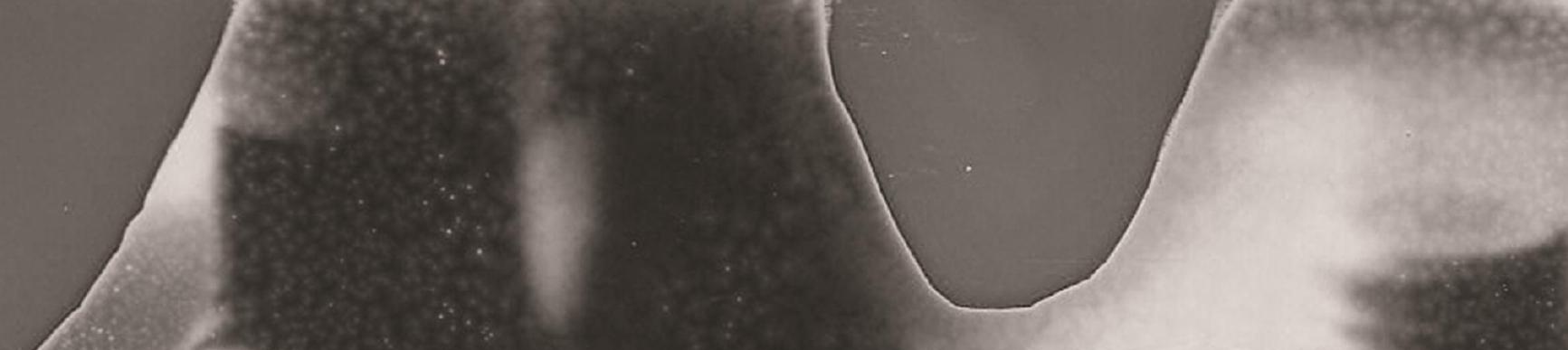

Summary et disculssio

6ing
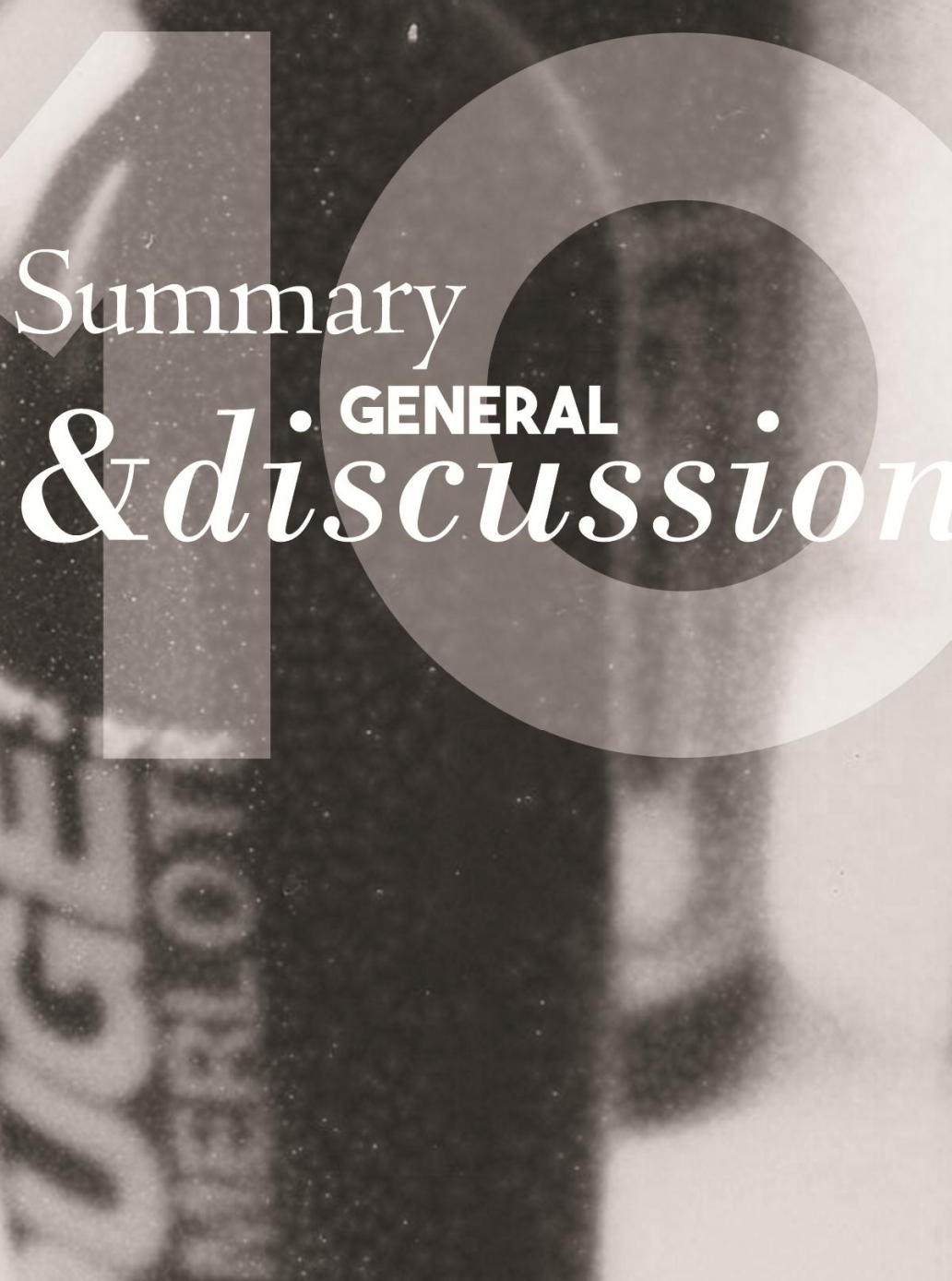

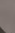
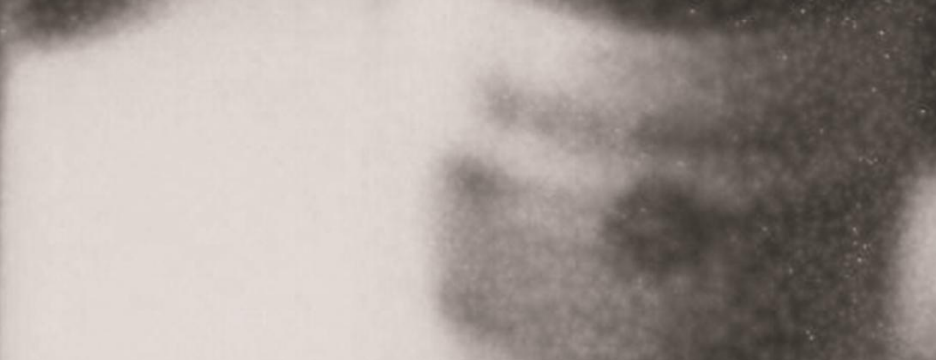


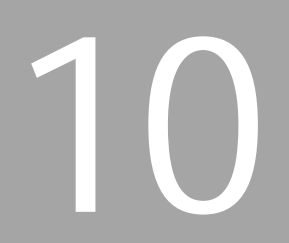

Summary \& General discussion 


\section{Summary \& general discussion}

Diabetes mellitus is a chronic, metabolic disease characterized by elevated levels of blood glucose $^{1}$, and is an established risk factor for coronary heart disease and ischaemic stroke ${ }^{2,3}$. It is affecting approximately 422 million people worldwide and has become a major public health concern $n^{4,5}$. Hyperglycaemia leads to reactive dicarbonyl compounds such as methylglyoxal ${ }^{6}$ and increased formation of advanced glycation endproducts (AGEs). To investigate the consequences of dicarbonyl compounds and AGEs for human health, sensitive and reliable analytical techniques are needed. The aim of the research described in this thesis was to develop analytical techniques to measure dicarbonyl compounds and AGEs and to apply these techniques in clinical studies.

\section{Advanced glycation endproducts; development of analytical techniques and its application}

In the early beginning of AGE research, AGEs were detected and quantified as a group of AGEs with fluorescent properties ${ }^{7,8}$. However, quantification of specific fluorescent AGEs such as crossline $e^{9}$ pentosidine ${ }^{10}$, vesperlysine $A, B$ and $C^{11}, M R X^{12}$ and pyrrolopyridinium ${ }^{11}$, was not possible with this technique. Later on, specific AGEs were quantified by enzyme-linked immunosorbent assays (ELISA) ,, $13-17^{2}$ but specificity, reproducibility and sensitivity of this technique were not optimal ${ }^{18-20}$. A better approach for the quantitative determination of AGEs is the use of a specific analytical technique.

In chapter 2A, a rapid and sensitive single-column high performance liquid chromatography (HPLC) method and application for the detection of protein bound pentosidine is described. Many techniques were used to quantify pentosidine ${ }^{21-27}$ with some disadvantages such as long run-times ${ }^{21,24,28}$, elaborate sample preparation ${ }^{29}$ or analysis on expensive equipment ${ }^{25,30}$. The described technique in chapter $2 \mathrm{~A}$ has overcome these shortcomings and presented a very clean chromatogram with a very stable retention-time and a run-to-run time of only 30 minutes. Moreover, the sample preparation is also suitable for the simultaneous analysis of protein-bound $\mathrm{N}^{\varepsilon}$-(carboxymethyl)|ysine (CML), $\mathrm{N}^{\varepsilon}$-(carboxyethyl)lysine (CEL), $\mathrm{N}^{\delta}$-(5-hydro-5-methyl-4imidazolon-2-yl)-ornithine (MG-H1) and lysine (chapter 2B). With this improved technique protein-bound pentosidine was analyzed in plasma samples of healthy volunteers and patients on peritoneal dialysis (PD). A 15 -fold increase of plasma protein-bound pentosidine was found in uremic patients when compared to healthy controls. 
In chapter 2B a sensitive, simple and highly selective method to determine protein-bound and free $\mathrm{CML}, \mathrm{CEL}$ and $\mathrm{MG}-\mathrm{H} 1$ in plasma by ultra-performance liquid chromatography (UPLC-M S/MS) is described.

Many techniques including ELISA ${ }^{7,13,15-17}$, HPLC ${ }^{31-34}$, gas chromatography mass spectrometry (GC-MS) ${ }^{18,35}$ and liquid chromatography mass spectrometry $(\mathrm{LC}-\mathrm{MS})^{17,25,34,36}$, have been described to determine AGEs in plasma and other matrices. However, all these techniques have several limitations. For the ELISA, standardization is difficult, immunoreactivity of the antibody and AGE epitope can be affected by numerous factors and sensitivity and specificity is often a matter of concern ${ }^{15}$. HPLC and LC-M S uses elaborate and expensive enzymatic hydrolysis ${ }^{25,32,34}$ which is not desirable for large cohort studies. Moreover, several analytical methods analyzed just one or two AGEs ${ }^{17,18,36}$. The described method in chapter 2B has overcome these limitations and presented a well validated, sensitive, simple and highly selective method to determine $C M L, C E L$ and MG-H1 in plasma. With this technique, protein-bound and free plasma AGEs were analyzed in healthy volunteers and patients on PD. Increased plasma levels of proteinbound and free AGEs were found to be increased in patients on PD as compared to healthy controls. This was in reasonable agreement with data described in literature ${ }^{36-41}$. The described method is suitable for studying AGEs in several different human and animal studies $^{42-49}$ and is a strong tool to investigate the putative effects of AGEs in the pathophysiology of different diseases.

In chapter 3, the analytical methods as described in chapter $2 \mathrm{~A}$ and $\mathrm{B}$, were used to investigate the associations of plasma AGEs with cardiovascular disease (CVD) in individuals with or without type 2 diabetes mellitus (T2DM). In this cross-sectional analysis of a combined sample of the Cohort on Diabetes and Atherosclerosis M aastricht (CODAM) and the Hoorn study, we found no associations of plasma protein-bound and free AGEs with glucose metabolism status and prevalent CVD. These results might be somewhat confusing since the putative effects of AGE accumulation on risk of CVD have been described previously ${ }^{50,51}$ and higher plasma AGE levels were found in type 1 diabetes mellitus (T1DM) ${ }^{52}$. However, given the cross-sectional design of the presented study, we cannot completely rule out the lack of associations of plasma AGEs with prevalent CVD. Possible lifestyle interventions, such as glucose-, blood-pressure- and lipid-lowering treatments ${ }^{50,53,54}$ and changes in dietary AGEs (due to diet interventions), may potentially influence circulating AGEs. Moreover, CM L has been found to be trapped in adipose tissues ${ }^{42,55}$ and consequently, plasma CML levels are lower in individuals with overweight compared to lean individuals ${ }^{55}$. Given the high prevalence of overweight and obesity in patients with T2DM, this phenomenon manifests more often in T2DM as compared to T1DM or lean individuals. 


\section{D-lactate as a reflection of methylglyoxal?}

Methylglyoxal (MGO), a highly reactive dicarbonyl compound generated by the spontaneous degradation of glycolytic intermediates, is a major precursor for AGEs and can potentially disrupt cellular functions. To investigate the glyoxalase pathway, in particular the detoxification of this M GO into D-lactate, there is need of a sensitive and highly specific method to determine D-lactate. Several different techniques have been described with some shortcomings such as low sensitivity ${ }^{56-58}$, large sample volume $e^{59-61}$, complex chromatographic systems $\mathrm{s}^{62-64}$ and long run times ${ }^{56,58,62,65}$. In chapter 4 a highly sensitive, specific and fast UPLC-M S/M S method to determine L- and D-lactate in plasma and urine is described. Without the need of cumbersome chiral stationary phases or complex chromatographic systems, a significant improvement over the methods described in literature was achieved. Because the origin of $D$-lactate may be derived from the production of intestinal bacteria and degradation of methylglyoxal by the glyoxalase pathway L- and D-lactate was determined in plasma and urine samples of controls, patients with inflammatory bowel disease (IBD) and patients with T2DM. Increased plasma and urinary levels of $\mathrm{L}$ - and $\mathrm{D}$-lactate in patients with IBD and T2DM compared to controls were found, but only significantly increased in patients with T2DM. Although these findings strongly suggest that the increased levels of D-lactate in patients with T2DM is a reflection of M GO, we cannot definitely exclude the possibility that D-lactate is a reflection of gut flora, or gut permeability.

In chapter 5, we have further explored the association between plasma D-lactate and insulin resistance. In this study the association of plasma D-lactate, as a reflection of M GO levels, and insulin resistance (HOM A2-IR) was investigated by multiple linear regression analysis. A positive association between plasma D-lactate and HOMA2-IR was found, independently of potential confounders. M oreover, an increase of plasma D-lactate levels in patients with T2DM was demonstrated. These results are in agreement with the results described in chapter 4 and suggest that M GO plays a role in insulin resistance. However, the question remained if D-lactate is a reflection of MGO levels. For answering this question, a direct measurement of MGO was needed.

\section{a-Oxoaldehydes and type 2 diabetes}

Numerous techniques have been described for the quantification of the reactive $\alpha$-oxoaldehydes glyoxal (GO), methylglyoxal (MGO) and 3-deoxyglucosone (3-DG) including reversed phase liquid chromatography with fluorescence or UV detection ${ }^{66-71}$ or liquid chromatography with mass spectrometry detection ${ }^{72-76}$. However, these techniques have several shortcomings such as large sample volume ${ }^{67,69,72,73}$, elaborate liquid-liquid extraction (LLE) or solid phase extraction (SPE) steps ${ }^{66,67,72-75}$ and long run-times ${ }^{66,68,71}$.

In chapter 6 a highly sensitive, specific and rapid ultra-performance liquid chromatography (UPLC) tandem mass spectrometry (MS/MS) method for the analysis of 
GO, M GO and 3-DG is described. A significant improvement over the methods described in the literature was achieved with the use of a stable isotope labeled internal standard. Sample preparation techniques, variations in sample treatment and storage and also the interval between blood draw and sample analysis can influence the free concentrations of $\alpha$-oxoaldehydes. Standardization of these conditions is necessary to obtain reliable and reproducible results.

Therefore, blood has to be withdrawn in EDTA tubes and subsequently derivatized as plasma with O-phenylenediamine (OPD) in the presence of perchloric acid (PCA). In particular, EDTA tubes with added sodium fluoride (NaF) should be avoided because inhibition of the enolase by $\mathrm{NaF}^{77}$ may lead to the accumulation of precursors of MGO and GO.

With this improved technique, increased plasma concentrations of GO, MGO and 3-DG were found in patients with T2DM as compared to healthy individuals. These results were in reasonable agreement with data described in literature ${ }^{69,78-80}$. Nevertheless, absolute concentrations differ between studies and are most likely due to differences in sample preparation and sample handling during storage. Moreover, these results support that Dlactate is most likely a reflection of the detoxification of M GO by the glyoxalase system as stated in chapter 4 and 5.

Patients with diabetes or impaired glucose metabolism are at higher risk for the development of micro- and macrovascular complications and CVD ${ }^{81}$. As a consequence of a disturbed glucose metabolism, glucose-derived reactive $\alpha$-oxoaldehydes are formed (chapter 6) and may lead to diabetic complications ${ }^{78,79,82,83}$. However, it is unknown to what extent the circulating $\alpha$-oxoaldehydes are affected after a postprandial glucose excursion. Postprandial glucose excursions have increasingly been recognized as a contributing factor to the increased risk of vascular complications ${ }^{84,85}$.

In chapter 7, we have investigated whether $\alpha$-oxoaldehydes are increased after a glucose load in individuals with normal and impaired glucose metabolism (NGM and IGM) and in patients with T2DM. Plasma $\alpha$-oxoaldehydes were determined in the fasting state and after an oral glucose tolerance test (OGTT), by UPLC-MS/MS (method described in chapter 6). Cross-sectional, linear analysis was performed in the CODAM study, consisting of 574 individuals.

This study demonstrated a positive and significant association of glucose metabolism status with higher $\alpha$-oxoaldehyde incremental area under the curve (iAUC), as calculated from an OGTT. This association was independent of potential confounders. Moreover, fasting plasma levels of MGO and 3-DG were predominantly higher in patients with T2DM. However, after adjustment for glucose, these associations disappeared completely, indicating glucose as a major source for these $\alpha$-oxoaldehydes. 
These results are in agreement with data described by Beisswenger et al. ${ }^{86}$, who found increased plasma levels of MGO and 3-DG in patients with T1DM after a postprandial glycemic excursion.

Since higher levels of $\alpha$-oxoaldehydes are directly associated with vascular complications $s^{44,87}$ and formed directly from glucose, postprandial periods may lead to an increased risk of vascular dysfunction. Consequently, fluctuations in blood glucose levels, in particular in patients with IGM or diabetes, may lead to increased $\alpha$-oxoaldehyde levels and thus increases risk for vascular complications and CVD. These findings underline the potential importance of dicarbonyl stress as a functional candidate to explain the increased risk of diabetic complications in individuals with postprandial hyperglycemia.

\section{Advanced glycation endproducts in foods}

Accumulation of AGEs in the body has been implicated in the pathogenesis of age-related diseases in particular in relation to diabetes, inflammation, neurodegenerative disorders and cardiovascular disease ${ }^{46,88-90}$. Since the processing and preparation of foods is also susceptible to AGE formation with high temperatures as a potent promoter ${ }^{91-94}$ dietary AGEs may influence the endogenous AGE pool. Animal studies have shown that some of these dietary AGEs are absorbed and have pathological effects, such as inducing insulin resistance ${ }^{92,95,96}$. In humans, dietary AGE intervention studies have shown to increase circulating AGEs and markers of inflammation and endothelial dysfunction, and to impair flow-mediated dilation and insulin sensitivity ${ }^{97-101}$. Nevertheless, many studies on dietary AGEs are based on semi-quantitative techniques, such as ELISA, and should be interpreted with care ${ }^{18,92}$. Therefore, a sensitive and reliable analytical method to determine the AGE content in foods was needed.

In chapter 8, a UPLC-MS/MS method for the determination of CML, CEL and MG-H1 in foods was validated. A database of AGE content in 190 food items, commonly consumed in a western diet, was presented. Overall, the food items which were heated, had long processing times, and were industrially conserved or products containing chocolate, nuts or grains were high in AGE content. Food items with a short heat processing time, low protein and/or carbohydrate content or high water content had low or negligible levels of AGEs. For CML, these data were in line with data from Hull et al. ${ }^{102}$ who presented a database of CML content in 257 food items as analyzed with the same technique. However, absolute concentrations differ, most likely due to differences in preparation techniques ${ }^{91,94,102-107}$. Three other major studies were described, but these studies were based on ELISA techniques ${ }^{107-109}$ and show inconsistent results. Since ELISA based techniques are sensitive for interference of the lipid matrix ${ }^{18}$ and steric hindrance of the antigen-antibody interaction as a consequence of matrix interferences ${ }^{92}$ these data should be interpreted with care.

The main advantages of the study, presented in chapter 8, are the use of a state-of-theart UPLC-tandem MS technique and the ability to analyze three major AGEs in one run. 
The presented AGE database and the validated analytical method are strong tools to further investigate the relevance of dietary AGEs in disease.

Intervention studies, with low and high AGE diets, to study the health effect of AGEs in much more detail are now possible.

However, whether dietary AGEs are substantially absorbed in the digestive tract and released into the circulation to contribute to any of these effects remains unclear. So far, studies investigating whether higher intake of AGEs is associated with circulating AGEs showed inconsistent results ${ }^{19,97-100,110-116}$, because most of these studies have been small, have not taken into account confounding factors or used different techniques to analyze AGEs.

In chapter 9, these considerations have been taken into account and the associations of dietary CM L, CEL and M G-H1 with plasma and urinary levels of their corresponding AGES were investigated. In 450 participants of the CODAM study, plasma and urinary levels of $\mathrm{CML}, \mathrm{CEL}$ and MG-H1 were determined, using the method described in chapter 2B. Dietary intake of $\mathrm{CM} \mathrm{L}, \mathrm{CEL}$ and $\mathrm{MG}-\mathrm{H} 1$ was estimated with the dietary database (chapter 8) and a food frequency questionnaire (FFQ). A positive and statistically significant association was found between dietary $\mathrm{CML}, \mathrm{CEL}$ and $\mathrm{MG}-\mathrm{H} 1$ and their corresponding free plasma and urinary levels. In addition, a positive but non-significant association was found between dietary AGEs and their corresponding protein bound plasma AGEs. These results are in line with previous studies ${ }^{97,98,100,111-113}$, but the current study expands on these findings in several important ways. The presented study used state-of-the-art UPLC-MS/MS technique to analyze three different dietary AGES and circulating AGEs. Statistical analysis was done in a large and well-defined cohort study to investigate the associations between dietary AGEs and plasma and urinary AGEs corrected for potential confounders. M oreover, a reliable database of AGEs (chapter 8) was used to calculate the habitual intake of dietary AGEs based on a FFQ. However, no detailed information about food preparation techniques, such as baking, grilling or frying, and also duration of this heating were described. Therefore, in ongoing studies we have implemented additional questions about food preparation and high AGE-content foods.

\section{Overview of dicarbonyl compounds and AGEs in body fluids}

With the developed techniques, described in this thesis, we were able to analyze dicarbonyl compounds, AGEs and related biomolecules in healthy and uremic patients, IBD patients and individuals with type 2 diabetes. An overview of the concentrations of these biomolecules in different disease states and body fluids, as presented in the different chapters of this thesis, is shown in table 10.1. 
Tabel 10.1 Overview of dicarbonyl compounds and AGEs in body fluids.

\begin{tabular}{|c|c|c|c|c|}
\hline Compound & Technique & Material & Subjects & Mean concentration \\
\hline $\begin{array}{l}\text { Pentosidine (protein- } \\
\text { bound) }\end{array}$ & $\begin{array}{l}\text { HPLC- } \\
\text { fluorescence }\end{array}$ & Heparin plasma & $\begin{array}{l}\text { Healthy volunteers: } \\
\text { Uremic patients: }\end{array}$ & $\begin{array}{l}0.21 \mathrm{pmol} / \mathrm{mg} \text { protein; } \\
\text { median }^{10} \\
3.05 \mathrm{pmol} / \mathrm{mg} \text { protein; } \\
\text { median }^{10}\end{array}$ \\
\hline CML (protein-bound) & UPLC-MS/MS & EDTA plasma & $\begin{array}{l}\text { Healthy volunteers: } \\
\text { Uremic patients: }\end{array}$ & $\begin{array}{l}3129 \mathrm{nmol} / \mathrm{L}^{\text {Chapter 2B }} \\
13832 \mathrm{nmol} / \mathrm{L}^{\text {Chapter 2B }}\end{array}$ \\
\hline CML (free) & UPLC-MS/MS & EDTA plasma & $\begin{array}{l}\text { Healthy volunteers: } \\
\text { Uremic patients: }\end{array}$ & $\begin{array}{l}68.5 \mathrm{nmol} / \mathrm{L}^{\text {Chapter } 2 B} \\
1084 \mathrm{nmol} / \mathrm{L}^{\text {Chapter 2B }}\end{array}$ \\
\hline CEL (protein-bound) & UPLC-MS/MS & EDTA plasma & $\begin{array}{l}\text { Healthy volunteers: } \\
\text { Uremic patients: }\end{array}$ & $\begin{array}{l}1902 \mathrm{nmol} / \mathrm{L}^{\text {Chapter 2B }} \\
2762 \mathrm{nmol} / \mathrm{L}^{\text {Chapter 2B }}\end{array}$ \\
\hline CEL (free) & UPLC-MS/MS & EDTA plasma & $\begin{array}{l}\text { Healthy volunteers: } \\
\text { Uremic patients: }\end{array}$ & $\begin{array}{l}104.5 \mathrm{nmol} / \mathrm{L}^{\text {Chapter 2B }} \\
1345 \mathrm{nmol} / \mathrm{L}^{\text {Chapter } 2 \mathrm{~B}}\end{array}$ \\
\hline MG-HI (protein-bound) & UPLC-MS/MS & EDTA plasma & $\begin{array}{l}\text { Healthy volunteers: } \\
\text { Uremic patients: }\end{array}$ & $\begin{array}{l}7800 \mathrm{nmo} / \mathrm{L}^{\text {Chapter } 2 \mathrm{~B}} \\
14105 \mathrm{nmol} / \mathrm{L}^{\text {Chapter 2B }}\end{array}$ \\
\hline MG-H1 (free) & UPLC-MS/MS & EDTA plasma & $\begin{array}{l}\text { Healthy volunteers: } \\
\text { Uremic patients: }\end{array}$ & $\begin{array}{l}409.1 \mathrm{nmol} / \mathrm{L}^{\text {Chapter 2B }} \\
4161 \mathrm{nmol} / \mathrm{L}^{\text {Chapter 2B }}\end{array}$ \\
\hline L-Lactate & UPLC-MS/MS & Urine & $\begin{array}{l}\text { Healthy volunteers: } \\
\text { IBD* patients: } \\
\text { T2DM** patients: }\end{array}$ & $\begin{array}{l}6.3 \mu \mathrm{mol} / \mathrm{mmol} \text { creat }^{117} \\
11.8 \mu \mathrm{mol} / \mathrm{mmol} \text { creat }^{117} \\
12.1 \mu \mathrm{mol} / \mathrm{mmol}^{112 e a t}{ }^{117}\end{array}$ \\
\hline D-Lactate & UPLC-MS/MS & Urine & $\begin{array}{l}\text { Healthy volunteers: } \\
\text { IBD* patients: } \\
\text { T2DM** patients: }\end{array}$ & $\begin{array}{l}1.1 \mu \mathrm{mol} / \mathrm{mmol} \text { creat }^{117} \\
3.1 \mu \mathrm{mol} / \mathrm{mmol} \text { creat }^{117} \\
3.4 \mu \mathrm{mol} / \mathrm{mmol} \text { creat }^{117}\end{array}$ \\
\hline L-Lactate & UPLC-MS/MS & EDTA plasma & $\begin{array}{l}\text { Healthy volunteers: } \\
\text { IBD* patients: } \\
\text { T2DM** patients: }\end{array}$ & $\begin{array}{l}1044.8 \mu \mathrm{mol} / \mathrm{L}^{117} \\
1172.4 \mu \mathrm{mol} / \mathrm{L}^{117} \\
1534.7 \mu \mathrm{mol} / \mathrm{L}^{117}\end{array}$ \\
\hline D-Lactate & UPLC-MS/MS & EDTA plasma & $\begin{array}{l}\text { Healthy volunteers: } \\
\text { IBD* patients: } \\
\text { T2DM** patients: }\end{array}$ & $\begin{array}{l}8.0 \mu \mathrm{mol} / \mathrm{L}^{117} \\
10.7 \mu \mathrm{mol} / \mathrm{L}^{117} \\
12.3 \mu \mathrm{mol} / \mathrm{L}^{117}\end{array}$ \\
\hline M ethylglyoxal & UPLC-M S/MS & EDTA plasma & $\begin{array}{l}\text { Healthy volunteers: } \\
\text { T2DM** patients: }\end{array}$ & $\begin{array}{l}212 \mathrm{nmol} / \mathrm{L}^{118} \\
277 \mathrm{nmol} / \mathrm{L}^{118}\end{array}$ \\
\hline Glyoxal & UPLC-MS/MS & EDTA plasma & $\begin{array}{l}\text { Healthy volunteers: } \\
\text { T2DM** patients: }\end{array}$ & $\begin{array}{l}406 \mathrm{nmol} / \mathrm{L}^{118} \\
514 \mathrm{nmol} / \mathrm{L}^{118}\end{array}$ \\
\hline 3-Deoxyglucosone & UPLC-MS/MS & EDTA plasma & $\begin{array}{l}\text { Healthy volunteers: } \\
\text { T2DM** patients: }\end{array}$ & $\begin{array}{l}1046 \mathrm{nmol} / \mathrm{L}^{118} \\
2217 \mathrm{nmol} / \mathrm{L}^{118}\end{array}$ \\
\hline
\end{tabular}

*IBD: patients with inflammatory bowel disease

**T2DM : patients with type 2 diabetes mellitus 


\section{Quantitative research: a critical appraisal}

In this thesis we have developed and used state-of-the-art techniques to quantify $\alpha$-oxoaldehydes, AGEs and other biomolecules in the AGE-pathway, in a broad range of sample matrices, to investigate their role in relation to disease.

A large part of our medical and scientific knowledge is based on quantitative measurements. Quantitative data, of AGEs, are used in many studies to answer important research questions about disease and health. However, it is important that researchers, in this field, are aware of methodological limitations that could bias this data and influence the findings of their study. Quantitative data, of AGEs, may be biased due to a number of factors, including pre-analytical processes, sample preparation and matrix effects.

The first step in quantitative research is the collection of a given sample, such as blood, plasma or urine. This first step can be a major source of variability and artefacts due to the instability of the compounds of interest. Compounds can be subjected to oxidation, aggregation or degradation as a consequence of poorly defined pre-analytical procedures. Sample handling and storage (e.g. choice of anticoagulant and collection tubes, time of incubation before plasma separation from blood cells, storage conditions and freezethaw cycles) and their effect on the stability of the compounds of interest should be well investigated $^{119-121}$.

After collecting the sample it has to be prepared for analysis. Many sample preparation methods have been described, including various solvent precipitation techniques, ultrafiltration, liquid-liquid extraction and solid-phase extraction methods ${ }^{25,36,122,123}$. For the measurement of circulating molecules, such as free AGEs, D-lactate or $\alpha$-oxoaldehydes in plasma, it is of utmost important to remove proteins by precipitation or ultra-filtration before analysis. The removal of these proteins prevents blockage of the LC-column and improves ion sufficiency during electrospray ionization detection ${ }^{124}$. On the contrary, protein-bound AGEs are determined after acid hydrolysis of the protein pellet and subsequently detected after derivatization. However, these sample preparation techniques can influence quantitative results by low metabolite coverage, low recovery and matrix effects ${ }^{122}$.

In this thesis several analytical methods for the detection of AGEs, D-lactate and $\alpha$-oxoaldehydes have been described. We have considered the methodological flaws, as described above. As described in previous chapters, stability, linearity, recovery and precision of the analytes were all well investigated and possible matrix effects were overcome by the use of stable-isotope internal standards. 


\section{Conclusions and future perspectives}

Since the introduction of liquid chromatography (LC) by Martin and Synge ${ }^{125}$ in 1941 , many important improvements were done to make this technique the strong analytical tool as we know it today. Several manufacturers have improved hardware and software and pushed the applicability of LC to the next level. In this thesis the application and strength of high-performance liquid chromatography (HPLC) and ultra-performance liquid chromatography (UPLC) for the detection of dicarbonyls and AGEs were explored. With the developed techniques, we were able to further investigate the role of AGES and related compounds in relation to disease. The main results of this thesis is the development of rapid, sensitive and selective LC and UPLC-tandem MS methods to analyze pentosidine, $\mathrm{N}^{\varepsilon}$-(carboxymethyl)lysine (CML), $\mathrm{N}^{\varepsilon}$-(carboxyethyl)lysine (CEL), $\mathrm{N}^{\delta}$-(5-hydro-5-methyl-4-imidazolon-2-yl)-ornithine (M G-H1), L-lactate, D-lactate, Glyoxal (GO), Methylglyoxal (MGO) and 3-Deoxyglucosone (3-DG). With the developed techniques we found that:

- Plasma concentrations of protein-bound pentosidine, CM L, CEL and M G-H1 and free CML, CEL and M G-H1 were significantly higher in uremic patients compared to healthy controls.

- Plasma D-lactate levels can be used as a reflection of MGO.

- Postprandial glucose excursions are related to an increase of $\alpha$-oxoaldehyde levels in individuals with impaired glucose metabolism (IGM ) and type 2 diabetes mellitus (T2DM).

- Higher intake of dietary protein-bound CM L, CEL and MG-H1 is associated with higher levels of free plasma and urinary CM L, CEL and MG-H1.

With the newly developed methods, as described in this thesis, we open the opportunity to investigate the glycation pathway in more detail. We already have shown that increased blood glucose levels, after postprandial glucose excursions or in patients with T2DM, plays an important role in the formation of $\alpha$-oxoaldehydes. Consequently, these precursors can lead to an increased endogenous formation of AGEs and may explain the increased risk for micro- and macrovascular complications. Furthermore, exogenous sources of AGEs, such as dietary AGEs, can also contribute to the circulating AGEs. However, whether these dietary AGEs have important implications for those who ingest a diet high in AGEs is still under debate. To further investigate the consequences of these dietary AGEs, well designed intervention studies are needed.

In conclusion, the analytical methods and results as described in this thesis, are key, to obtain detailed information about the effects of dietary AGEs and endogenous AGEs for health and disease. 


\section{References}

Turner, R. C., Mann, J. I., Simpson, R. D., Harris, E. \& Maxwell, R. Fasting hyperglycaemia and relatively unimpaired meal responses in mild diabetes. Clinical endocrinology 6, 253-264 (1977).

Spencer, E. A. et al. Diabetes and modifiable risk factors for cardiovascular disease: the prospective Million Women Study. Eur J Epidemiol 23, 793-799, doi:10.1007/s10654-008-9298-3 (2008).

Schramm, T. K. et al. Diabetes patients requiring glucose-lowering therapy and nondiabetics with a prior myocardial infarction carry the same cardiovascular risk: a population study of 3.3 million people. Circulation 117, 1945-1954, doi:10.1161/circulationaha.107.720847 (2008).

WHO. Diabetes: Fact sheet no. 310, (2014).

WHO. Diabetes: Fact sheet no. 312, (2015).

Brownlee, M. Biochemistry and molecular cell biology of diabetic complications. Nature 414, 813-820 (2001)

Munch, G. et al. Determination of advanced glycation end products in serum by fluorescence spectroscopy and competitive ELISA. European journal of clinical chemistry and clinical biochemistry : journal of the Forum of European Clinical Chemistry Societies 35, 669-677 (1997).

Monnier, V. M., Kohn, R. R. \& Cerami, A. Accelerated age-related browning of human collagen in diabetes mellitus. Proc Natl Acad Sci U SA 81, 583-587 (1984).

Obayashi, H. et al. Formation of crossline as a fluorescent advanced glycation end product in vitro and in vivo. Biochem Biophys Res Commun 226, 37-41, doi:10.1006/bbrc.1996.1308 (1996).

Scheijen, J. L., van de Waarenburg, M. P., Stehouwer, C. D. \& Schalkwijk, C. G. Measurement of pentosidine in human plasma protein by a single-column high-performance liquid chromatography method with fluorescence detection. Journal of chromatography. B, Analytical technologies in the biomedical and life sciences 877, 610-614, doi:10.1016/j.jchromb.2009.01.022 (2009).

Nakamura, K., Nakazawa, Y. \& lenaga, K. Acid-stable fluorescent advanced glycation end products: vesperlysines $A, B$, and $C$ are formed as crosslinked products in the Maillard reaction between lysine or proteins with glucose. Biochem Biophys Res Commun 232, 227-230, doi:10.1006/bbrc.1997.6262 (1997).

Osawa, T. \& Kato, Y. Protective role of antioxidative food factors in oxidative stress caused by hyperglycemia. Ann N Y Acad Sci 1043, 440-451, doi:10.1196/annals.1333.050 (2005).

Sanaka, T. et al. Plasma pentosidine levels measured by a newly developed method using ELISA in patients with chronic renal failure. Nephron 91, 64-73 (2002).

Izuhara, Y. et al. A sensitive and specific ELISA for plasma pentosidine. Nephrol Dial Transplant 14, 576-580 (1999).

Mitsuhashi, T., Vlassara, H., Founds, H. W. \& Li, Y. M. Standardizing the immunological measurement of advanced glycation endproducts using normal human serum. J Immunol Methods 207, 79-88 (1997).

Takayama, F. et al. Immunohistochemical detection of imidazolone and N(epsilon)(carboxymethyl)lysine in aortas of hemodialysis patients. Cellular and molecular biology 44, 11011109 (1998).

Srey, C. et al. Immunochemical and mass spectrometric analysis of Nepsilon-(carboxymethyl)/ysine content of AGE-BSA systems prepared with and without selected antiglycation agents. Journal of agricultural and food chemistry 58, 11955-11961, doi:10.1021/jf102543g (2010).

Charissou, A., Ait-Ameur, L. \& Birlouez-Aragon, I. Evaluation of a gas chromatography/mass spectrometry method for the quantification of carboxymethyllysine in food samples. J Chromatogr A 1140, 189-194, doi:10.1016/j.chroma.2006.11.066 (2007). 
Tareke, E., Forslund, A., Lindh, C. H., Fahlgren, C. \& Ostman, E. Isotope dilution ESI-LC-MS/MS for quantification of free and total Nepsilon-(1-Carboxymethyl)-L-Lysine and free Nepsilon-(1Carboxyethyl)-L-Lysine: comparison of total Nepsilon-(1-Carboxymethyl)-L-Lysine levels measured with new method to ELISA assay in gruel samples. Food Chem 141, 4253-4259, doi:10.1016/j.foodchem.2013.07.003 (2013).

Delatour, T. et al. Analysis of advanced glycation endproducts in dairy products by isotope dilution liquid chromatography-electrospray tandem mass spectrometry. The particular case of carboxymethyllysine. J Chromatogr A 1216, 2371-2381 (2009).

Odetti, P., Fogarty, J., Sell, D. R. \& Monnier, V. M. Chromatographic quantitation of plasma and erythrocyte pentosidine in diabetic and uremic subjects. Diabetes 41, 153-159 (1992).

Mikulikova, K., Eckhardt, A., Kunes, J., Zicha, J. \& Miksik, I. Advanced glycation end-product pentosidine accumulates in various tissues of rats with high fructose intake. Physiol Res 57, 89-94 (2008).

Spacek, P. \& Adam, M. Pentosidine in osteoarthritis: HPLC determination in body fluids and in tissues. Rheumatol Int 26, 923-927 (2006).

Wilker, S. C., Chellan, P., Arnold, B. M. \& Nagaraj, R. H. Chromatographic quantification of argpyrimidine, a methylglyoxal-derived product in tissue proteins: comparison with pentosidine. Anal Biochem 290, 353-358 (2001).

Thornalley, P. J. et al. Quantitative screening of advanced glycation endproducts in cellular and extracellular proteins by tandem mass spectrometry. Biochem J 375, 581-592 (2003).

Taneda, S. \& M onnier, V. M. ELISA of pentosidine, an advanced glycation end product, in biological specimens. Clin Chem 40, 1766-1773 (1994).

Takahashi, M., Hoshino, H., Kushida, K., Kawana, K. \& Inoue, T. Direct quantification of pentosidine in urine and serum by HPLC with column switching. Clin Chem 42, 1439-1444 (1996).

Slowik-Zylka, D. et al. A sensitive and specific HPLC method for the determination of total pentosidine concentration in plasma. J Biochem Biophys M ethods 61, 313-329 (2004).

Floridi, A., Trizza, V., Paolotti, P. \& Lucarelli, C. Analytical strategy for the assessment of the protein glycation status in uremic patients by high-performance liquid chromatography. J Chromatogr A 846, 65-71 (1999).

Ahmed, N. \& Thornalley, P. J. Quantitative screening of protein biomarkers of early glycation, advanced glycation, oxidation and nitrosation in cellular and extracellular proteins by tandem mass spectrometry multiple reaction monitoring. Biochem Soc Trans 31, 1417-1422 (2003).

Ahmed, N. \& Thornalley, P. J. Chromatographic assay of glycation adducts in human serum albumin glycated in vitro by derivatization with 6 -aminoquinolyl-N-hydroxysuccinimidyl-carbamate and intrinsic fluorescence. Biochem J 364, 15-24 (2002).

Ahmed, N., Argirov, O. K., Minhas, H. S., Cordeiro, C. A. \& Thornalley, P. J. Assay of advanced glycation endproducts (AGEs): surveying AGEs by chromatographic assay with derivatization by 6 aminoquinolyl-N-hydroxysuccinimidyl-carbamate and application to Nepsilon-carboxymethyl-lysineand Nepsilon-(1-carboxyethyl)lysine-modified albumin. Biochem J 364, 1-14 (2002).

Hartkopf, J. \& Pahlke, C. Determination of N-carboxymethyllysine by a reversed-phase highperformance liquid chromatography method. J Chromatogr A 672, 242-246 (1994).

Ahmed, M. U., Thorpe, S. R. \& Baynes, J. W. Identification of $\mathrm{N}$ epsilon-carboxymethyllysine as a degradation product of fructoselysine in glycated protein. J Biol Chem 261, 4889-4894 (1986).

Anderson, M. M. \& Heinecke, J. W. Production of N(epsilon)-(carboxymethyl)lysine is impaired in mice deficient in NADPH oxidase: a role for phagocyte-derived oxidants in the formation of advanced glycation end products during inflammation. Diabetes 52, 2137-2143 (2003). 
36

Teerlink, T., Barto, R., Ten Brink, H. J. \& Schalkwijk, C. G. Measurement of Nepsilon(carboxymethyl)lysine and Nepsilon-(carboxyethyl)/ysine in human plasma protein by stable-isotopedilution tandem mass spectrometry. Clin Chem 50, 1222-1228 (2004).

Degenhardt, T. P. et al. Technical note. The serum concentration of the advanced glycation endproduct N epsilon-(carboxymethyl)lysine is increased in uremia. Kidney Int 52, 1064-1067 (1997).

Weiss, M. F. et al. Mechanisms for the formation of glycoxidation products in end-stage renal disease. Kidney Int 57, 2571-2585, doi:10.1046/j.1523-1755.2000.00117.x (2000).

Agalou, S., Ahmed, N., Dawnay, A. \& Thornalley, P. J. Removal of advanced glycation end products in clinical renal failure by peritoneal dialysis and haemodialysis. Biochem Soc Trans 31, 1394-1396 (2003).

Thornalley, P. J. Glycation free adduct accumulation in renal disease: the new AGE. Pediatr Nephrol 20, 1515-1522 (2005).

Agalou, S., Ahmed, N., Thornalley, P. J. \& Dawnay, A. Advanced glycation end product free adducts are cleared by dialysis. Ann N Y Acad Sci 1043, 734-739 (2005).

2 Gaens, K. H. et al. Nepsilon-(carboxymethyl)lysine-receptor for advanced glycation end product axis is a key modulator of obesity-induced dysregulation of adipokine expression and insulin resistance. Arteriosclerosis, thrombosis, and vascular biology 34, 1199-1208, doi:10.1161/ATVBAHA.113.302281 (2014).

Gaens, K. H. et al. Endogenous formation of Nepsilon-(carboxymethyl)lysine is increased in fatty livers and induces inflammatory markers in an in vitro model of hepatic steatosis. J Hepatol 56, 647655, doi:10.1016/j.jhep.2011.07.028 (2012).

Brouwers, 0 . et al. Overexpression of glyoxalase-I reduces hyperglycemia-induced levels of advanced glycation endproducts and oxidative stress in diabetic rats. J Biol Chem (2011).

van Eupen, M. G. et al. Plasma levels of advanced glycation endproducts are associated with type 1 diabetes and coronary artery calcification. Cardiovasc Diabetol 12, 149, doi:10.1186/1475-2840-12149 (2013).

Nin, J. W. et al. Higher plasma levels of advanced glycation end products are associated with incident cardiovascular disease and all-cause mortality in type 1 diabetes: a 12-year follow-up study. Diabetes Care 34, 442-447, doi:10.2337/dc10-1087 (2011).

Gopal, P. et al. Plasma advanced glycation end-products and skin autofluorescence are increased in COPD. The European respiratory journal 43, 430-438, doi:10.1183/09031936.00135312 (2014).

Hanssen NM, Engelen L, Ferreira I, Scheijen JL, Huijberts MS, van Greevenbroek M M, et al. Plasma levels of advanced glycation endproducts are not independently associated with cardiovascular disease in individuals with or without type 2 diabetes: the Hoorn and CODAM Studies. J Clin Endocrinol M etab (2013).

Hanssen, N. M. et al. Plasma advanced glycation endproducts are associated with incident cardiovascular events in individuals with type 2 diabetes: a case-cohort study with a median followup of 10 years (EPIC-NL). Diabetes, doi:10.2337/db13-1864 (2014).

Hanssen, N. M. et al. Plasma advanced glycation end products are associated with incident cardiovascular events in individuals with type 2 diabetes: a case-cohort study with a median followup of 10 years (EPIC-NL). Diabetes 64, 257-265, doi:10.2337/db13-1864 (2015).

Hanssen, N. M. et al. Higher levels of advanced glycation endproducts in human carotid atherosclerotic plaques are associated with a rupture-prone phenotype. Eur Heart J 35, 1137-1146, doi:10.1093/eurheartj/eht402 (2014).

van Eupen, M. G. et al. Plasma levels of advanced glycation endproducts are associated with type 1 diabetes and coronary artery calcification. Cardiovasc Diabetol 12, 149, doi:10.1186/1475-2840-12149 (2013). 
Beisswenger, P. J., Howell, S. K., Touchette, A. D., Lal, S. \& Szwergold, B. S. Metformin reduces systemic methylglyoxal levels in type 2 diabetes. Diabetes 48, 198-202 (1999).

Nangaku, M. et al. Anti-hypertensive agents inhibit in vivo the formation of advanced glycation end products and improve renal damage in a type 2 diabetic nephropathy rat model. J Am Soc Nephrol 14, 1212-1222 (2003).

Gaens, K. H. et al. Protein-Bound Plasma Nepsilon-(Carboxymethyl)lysine Is Inversely Associated With Central Obesity and Inflammation and Significantly Explain a Part of the Central Obesity-Related Increase in Inflammation: The Hoorn and CODAM Studies. Arterioscler Thromb Vasc Biol 35, 27072713, doi:10.1161/atvbaha.115.306106 (2015). chromatographic assay of lactic, pyruvic and acetic acids and lactic acid stereoisomers in calf feces, rumen fluid and urine. J Chromatogr B Analyt Technol Biomed Life Sci 805, 347-351 (2004).

Franco, E. J., Hofstetter, H. \& Hofstetter, O. Determination of lactic acid enantiomers in human urine by high-performance immunoaffinity LC-MS. J Pharm Biomed Anal 49, 1088-1091 (2009).

Tan, L., Wang, Y., Liu, X., Ju, H. \& Li, J. Simultaneous determination of I- and d-lactic acid in plasma by capillary electrophoresis. J Chromatogr B Analyt Technol Biomed Life Sci 814, 393-398 (2005).

\section{Brandt, R. B., Siegel, S. A., Waters, M. G. \& Bloch, M. H. Spectrophotometric assay for D-(-)-lactate in} plasma. Anal Biochem 102, 39-46 (1980).

Marti, R. et al. Determination of D-lactate by enzymatic methods in biological fluids: study of interferences. Clin Chem 43, 1010-1015 (1997).

1 Inoue, Y., Shinka, T., Ohse, M., Ikawa, H. \& Kuhara, T. Application of optical isomer analysis by diastereomer derivatization GC/MS to determine the condition of patients with short bowel syndrome. J Chromatogr B Analyt Technol Biomed Life Sci 838, 37-42 (2006).

Hasegawa, H. et al. Determination of serum D-lactic and L-lactic acids in normal subjects and diabetic patients by column-switching HPLC with pre-column fluorescence derivatization. Anal Bioanal Chem 377, 886-891 (2003).

63 Fukushima, T. et al. Simultaneous determination of D-lactic acid and 3-hydroxybutyric acid in rat plasma using a column-switching HPLC with fluorescent derivatization with 4-nitro-7-piperazino2,1,3-benzoxadiazole (NBD-PZ). Biomed Chromatogr 15, 189-195 (2001).

Ichihara, H., Fukushima, T. \& Imai, K. Enantiomeric determination of D- and L-lactate in rat serum using high-performance liquid chromatography with a cellulose-type chiral stationary phase and fluorescence detection. Anal Biochem 269, 379-385 (1999). Cevasco, G., Piatek, A. M., Scapolla, C. \& Thea, S. A simple, sensitive and efficient assay for the determination of $D$ - and L-lactic acid enantiomers in human plasma by high-performance liquid chromatography. J Chromatogr A 1218, 787-792 (2011).

Yamada, H. et al. Increase in 3-deoxyglucosone levels in diabetic rat plasma. Specific in vivo determination of intermediate in advanced M aillard reaction. J Biol Chem 269, 20275-20280 (1994). M cLellan, A. C., Phillips, S. A. \& Thornalley, P. J. The assay of methylglyoxal in biological systems by derivatization with 1,2-diamino-4,5-dimethoxybenzene. Anal Biochem 206, 17-23 (1992). quantification of the glucose degradation product glucosone in peritoneal dialysis fluids by

HPLC/DAD/M SM S. J Chromatogr B Analyt Technol Biomed Life Sci 878, 877-882 (2010).
Nemet, I., Varga-Defterdarovic, L. \& Turk, Z. Preparation and quantification of methylglyoxal in human plasma using reverse-phase high-performance liquid chromatography. Clin Biochem 37, 875881 (2004). 
Neng, N. R., Cordeiro, C. A. A., Freire, A. P. \& Nogueira, J. M. F. Determination of glyoxal and methylglyoxal in environmental and biological matrices by stir bar sorptive extraction with in-situ derivatization. J Chromatogr A 1169, 47-52 (2007).

Dhar, A., Desai, K., Liu, J. \& Wu, L. Methylglyoxal, protein binding and biological samples: are we getting the true measure? J Chromatogr B Analyt Technol Biomed Life Sci 877, 1093-1100 (2009).

Han, Y. et al. Plasma methylglyoxal and glyoxal are elevated and related to early membrane alteration in young, complication-free patients with Type 1 diabetes. Mol Cell Biochem 305, 123-131 (2007).

Nakayama, K. et al. Plasma alpha-oxoaldehyde levels in diabetic and nondiabetic chronic kidney disease patients. Am J Nephrol 28, 871-878 (2008).

Mittelmaier, S., Funfrocken, M., Fenn, D., Berlich, R. \& Pischetsrieder, M. Quantification of the six major alpha-dicarbonyl contaminants in peritoneal dialysis fluids by UHPLC/DAD/M SMS. Anal Bioanal Chem 401, 1183-1193 (2011).

Randell, E. W., Vasdev, S. \& Gill, V. Measurement of methylglyoxal in rat tissues by electrospray ionization mass spectrometry and liquid chromatography. J Pharmacol Toxicol Methods 51, 153-157 (2005).

Kampf, C. J., Bonn, B. \& Hoffmann, T. Development and validation of a selective HPLC-ESI-MS/MS method for the quantification of glyoxal and methylglyoxal in atmospheric aerosols (PM2.5). Anal Bioanal Chem 401, 3115-3124 (2011).

Mikesh, L. M. \& Bruns, D. E. Stabilization of glucose in blood specimens: mechanism of delay in fluoride inhibition of glycolysis. Clin Chem 54, 930-932 (2008).

8 Odani, H., Shinzato, T., M atsumoto, Y., Usami, J. \& Maeda, K. Increase in three alpha, beta-dicarbonyl compound levels in human uremic plasma: specific in vivo determination of intermediates in advanced M aillard reaction. Biochem Biophys Res Commun 256, 89-93 (1999).

Lu, J. et al. Increased plasma methylglyoxal level, inflammation, and vascular endothelial dysfunction in diabetic nephropathy. Clin Biochem 44, 307-311 (2011).

Fleming, $T$. et al. Is diabetes an acquired disorder of reactive glucose metabolites and their intermediates? Diabetologia 55, 1151-1155, doi:10.1007/s00125-012-2452-1 (2012).

Forbes, J. M. \& Cooper, M. E. Mechanisms of diabetic complications. Physiol Rev 93, 137-188,

doi:10.1152/physrev.00045.2011 (2013). an old paradigm. Diabetes 48, 1-9 (1999).

Ogawa, S. et al. Methylglyoxal is a predictor in type 2 diabetic patients of intima-media thickening and elevation of blood pressure. Hypertension 56, 471-476 (2010).

de Vegt, F. et al. Hyperglycaemia is associated with all-cause and cardiovascular mortality in the Hoorn population: the Hoorn Study. Diabetologia 42, 926-931, doi:10.1007/s001250051249 (1999).

Beisswenger, P., Heine, R. J., Leiter, L. A., Moses, A. \& Tuomilehto, J. Prandial glucose regulation in the glucose triad: emerging evidence and insights. Endocrine 25, 195-202, doi:10.1385/endo:25:3:195 (2004).

Beisswenger, P. J. et al. alpha-Dicarbonyls increase in the postprandial period and reflect the degree of hyperglycemia. Diabetes Care 24, 726-732 (2001).

Brouwers, 0. et al. Glyoxalase-1 overexpression reduces endothelial dysfunction and attenuates early renal impairment in a rat model of diabetes. Diabetologia 57, 224-235, doi:10.1007/s00125013-3088-5 (2014).

Basta, G., Schmidt, A. M. \& De Caterina, R. Advanced glycation end products and vascular inflammation: implications for accelerated atherosclerosis in diabetes. Cardiovasc Res 63, 582-592 (2004). 
Baumann, M. et al. N epsilon-(carboxymethyl)lysine during the early development of hypertension. Ann N Y Acad Sci 1126, 201-204 (2008).

Goldin, A., Beckman, J. A., Schmidt, A. M. \& Creager, M. A. Advanced glycation end products: sparking the development of diabetic vascular injury. Circulation 114, 597-605 (2006).

Pei-Chun Chao, C.-c. H., Mei-chin Yin. Analysis of glycative products in sauces and sauce-treated foods. Food Chemistry 113, 262-266 (2009).

Poulsen, M. W. et al. Advanced glycation endproducts in food and their effects on health. Food Chem Toxicol 60, 10-37, doi:10.1016/j.fct.2013.06.052 (2013).

Wellner, A., Huettl, C. \& Henle, T. Formation of M aillard reaction products during heat treatment of carrots. Journal of agricultural and food chemistry 59, 7992-7998, doi:10.1021/jf2013293 (2011).

Zhang, G., Huang, G., Xiao, L. \& Mitchell, A. E. Determination of advanced glycation endproducts by LC-M S/MS in raw and roasted almonds (Prunus dulcis). Journal of agricultural and food chemistry $\mathbf{5 9}$, 12037-12046, doi:10.1021/jf202515k (2011).

Guo, Q. et al. Methylglyoxal contributes to the development of insulin resistance and salt sensitivity in Sprague-Dawley rats. J Hypertens 27, 1664-1671, doi:10.1097/HJH.0b013e32832c419a (2009).

Vlassara, $H$. et al. Exogenous advanced glycosylation end products induce complex vascular dysfunction in normal animals: a model for diabetic and aging complications. Proc Natl Acad Sci U SA 89, 12043-12047 (1992).

Birlouez-Aragon, I. et al. A diet based on high-heat-treated foods promotes risk factors for diabetes mellitus and cardiovascular diseases. Am J Clin Nutr 91, 1220-1226, doi:10.3945/ajcn.2009.28737 (2010).

Mark, A. B. et al. Consumption of a Diet Low in Advanced Glycation Endproducts for 4 weeks Improves Insulin Sensitivity in Overweight Women. Diabetes Care, doi:10.2337/dc13-0842 (2013).

Uribarri, J. et al. Diet-derived advanced glycation end products are major contributors to the body's AGE pool and induce inflammation in healthy subjects. Annals of the New York Academy of Sciences 1043, 461-466, doi:10.1196/annals.1333.052 (2005).

Uribarri, J. et al. Single oral challenge by advanced glycation end products acutely impairs endothelial function in diabetic and nondiabetic subjects. Diabetes Care 30, 2579-2582, doi:10.2337/dc07-0320 (2007).

Uribarri, J. et al. Restriction of advanced glycation end products improves insulin resistance in human type 2 diabetes: potential role of AGER1 and SIRT1. Diabetes Care 34, 1610-1616, doi:10.2337/dc110091 (2011).

Hull, G. L. J., Woodside, J. V., Ames, J. M. \& Cuskelly, G. J. Nع-(carboxymethyl)lysine content of foods commonly consumed in a Western style diet. Food Chemistry 131, 170-174 (2012).

Ahmed, N. et al. Assay of advanced glycation endproducts in selected beverages and food by liquid chromatography with tandem mass spectrometric detection. M olecular nutrition \& food research $\mathbf{4 9}$, 691-699, doi:10.1002/mnfr.200500008 (2005).

Assar, S. H., Moloney, C., Lima, M., Magee, R. \& Ames, J. M. Determination of Nepsilon(carboxymethyl)lysine in food systems by ultra performance liquid chromatography-mass spectrometry. Amino acids 36, 317-326, doi:10.1007/s00726-008-0071-4 (2009).

Chen, G. \& Smith, J. S. Determination of advanced glycation endproducts in cooked meat products. Food Chem 168, 190-195, doi:10.1016/j.foodchem.2014.06.081 (2015).

Hegele, J. et al. Evaluating the extent of protein damage in dairy products: simultaneous determination of early and advanced glycation-induced lysine modifications. Ann N Y Acad Sci 1126, 300-306 (2008). 
107

Uribarri, J. et al. Advanced glycation end products in foods and a practical guide to their reduction in the diet. Journal of the American Dietetic Association 110, $911-916$ e912, doi:10.1016/j.jada.2010.03.018 (2010).

108 Goldberg, T. et al. Advanced glycoxidation end products in commonly consumed foods. Journal of the American Dietetic Association 104, 1287-1291, doi:10.1016/j.jada.2004.05.214 (2004).

109 Takeuchi, M. et al. Assessment of the concentrations of various advanced glycation end-products in beverages and foods that are commonly consumed in Japan. PLOS One 10, e0118652, doi:10.1371/journal.pone.0118652 (2015).

110 Poulsen, M. W. et al. Effect of dietary advanced glycation end products on postprandial appetite, inflammation, and endothelial activation in healthy overweight individuals. European journal of nutrition 53, 661-672, doi:10.1007/s00394-013-0574-y (2014).

111 Klenovics, K. S. et al. Advanced glycation end products in infant formulas do not contribute to insulin resistance associated with their consumption. PLoS One 8, e53056, doi:10.1371/journal.pone.0053056 (2013).

112 Sebekova, K. et al. Plasma concentration and urinary excretion of $\mathrm{N}$ epsilon-(carboxymethyl)lysine in breast milk- and formula-fed infants. Ann N Y Acad Sci 1126, 177-180, doi:10.1196/annals.1433.049 (2008).

113 Macias-Cervantes, M. H. et al. Effect of an advanced glycation end product-restricted diet and exercise on metabolic parameters in adult overweight men. Nutrition 31, 446-451, doi:10.1016/j.nut.2014.10.004 (2015).

114 Chao, P. C., Huang, C. N., Hsu, C. C., Yin, M. C. \& Guo, Y. R. Association of dietary AGEs with circulating AGEs, glycated LDL, IL-1alpha and MCP-1 levels in type 2 diabetic patients. European journal of nutrition 49, 429-434, doi:10.1007/s00394-010-0101-3 (2010).

115 Uribarri, J. et al. Dietary glycotoxins correlate with circulating advanced glycation end product levels in renal failure patients. Am J Kidney Dis 42, 532-538 (2003).

116 Negrean, M. et al. Effects of low- and high-advanced glycation endproduct meals on macro- and microvascular endothelial function and oxidative stress in patients with type 2 diabetes mellitus. Am J Clin Nutr 85, 1236-1243 (2007).

117 Scheijen, J. L. et al. $L(+)$ and $D(-)$ lactate are increased in plasma and urine samples of type 2 diabetes as measured by a simultaneous quantification of $L(+)$ and $D(-)$ lactate by reversed-phase liquid chromatography tandem mass spectrometry. Exp Diabetes Res 2012, 234812, doi:10.1155/2012/234812 (2012).

118 Scheijen, J. L. \& Schalkwijk, C. G. Quantification of glyoxal, methylglyoxal and 3-deoxyglucosone in blood and plasma by ultra performance liquid chromatography tandem mass spectrometry: evaluation of blood specimen. Clinical chemistry and laboratory medicine : CCLM / FESCC, 1-7, doi:10.1515/cclm-2012-0878 (2013).

119 Jobard, E. et al. A Systematic Evaluation of Blood Serum and Plasma Pre-Analytics for M etabolomics Cohort Studies. Int J M ol Sci 17, doi:10.3390/ijms17122035 (2016).

120 Yin, P., Lehmann, R. \& Xu, G. Effects of pre-analytical processes on blood samples used in metabolomics studies. Anal Bioanal Chem 407, 4879-4892, doi:10.1007/s00216-015-8565-x (2015).

121 Luque-Garcia, J. L. \& Neubert, T. A. Sample preparation for serum/plasma profiling and biomarker identification by mass spectrometry. J Chromatogr A 1153, 259-276, doi:10.1016/j.chroma.2006.11.054 (2007).

122 Sitnikov, D. G., Monnin, C. S. \& Vuckovic, D. Systematic Assessment of Seven Solvent and Solid-Phase Extraction Methods for Metabolomics Analysis of Human Plasma by LC-MS. Scientific reports $\mathbf{6}$, 38885, doi:10.1038/srep38885 (2016). 
123 Jemal, M., Teitz, D., Ouyang, Z. \& Khan, S. Comparison of plasma sample purification by manual liquid-liquid extraction, automated 96-well liquid-liquid extraction and automated 96-well solidphase extraction for analysis by high-performance liquid chromatography with tandem mass spectrometry. J Chromatogr B Biomed Sci Appl 732, 501-508 (1999).

124 Rico, E., Gonzalez, O., Blanco, M. E. \& Alonso, R. M. Evaluation of human plasma sample preparation protocols for untargeted metabolic profiles analyzed by UHPLC-ESI-TOF-MS. Anal Bioanal Chem 406, 7641-7652, doi:10.1007/s00216-014-8212-y (2014).

125 Martin, A. J. \& Synge, R. L. Separation of the higher monoamino-acids by counter-current liquid-liquid extraction: the amino-acid composition of wool. The Biochemical journal 35, 91-121 (1941). 

SAMENVATTING

valorization curriculum vitae

\section{SCIENTIFIC OUTPUT}

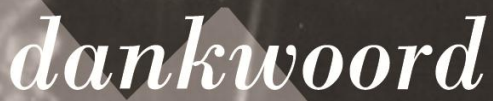

Ry.
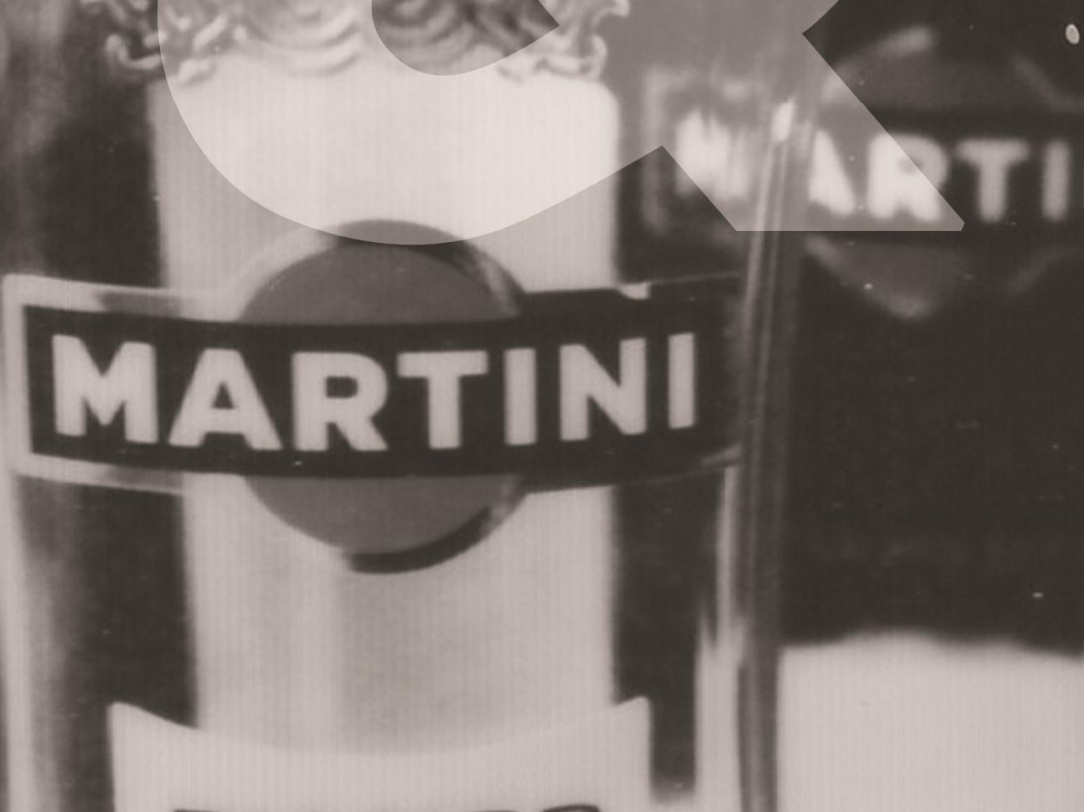

BIANCO
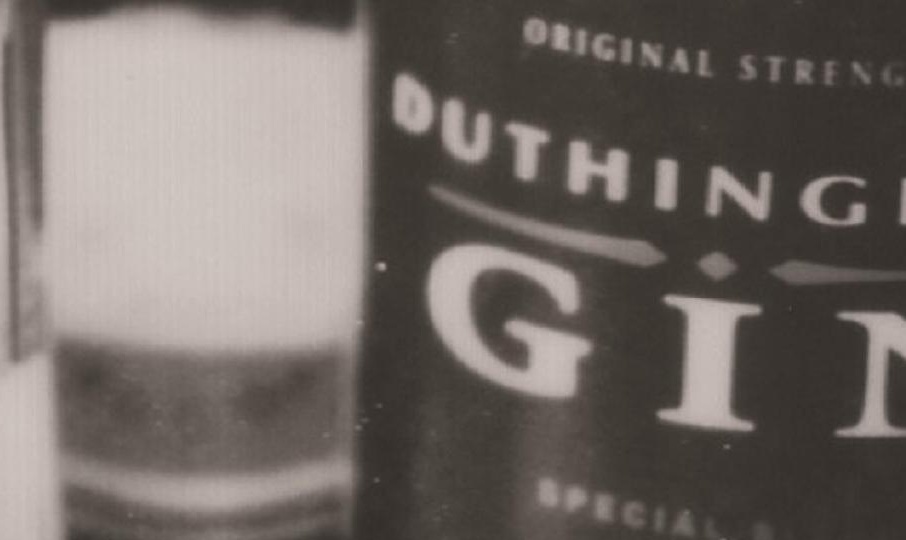

Asticia

Salceord voor

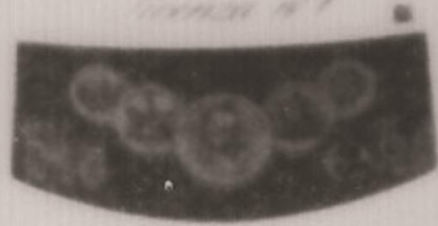
Qoetinchem 

Samenvatting 



\section{Samenvatting}

\section{Hart- en vaatziekten, obesitas en diabetes}

Hart- en vaatziekten, obesitas en diabetes kunnen leiden tot gezondheids-problemen of een kortere levensverwachting. Wereldwijd hebben 1,9 miljard mensen overgewicht (hiervan hebben 600 miljoen mensen obesitas) en 422 miljoen mensen diabetes. In 2012 overleden 1,5 miljoen mensen aan de gevolgen van diabetes. In Nederland heeft ongeveer de helft van alle volwassenen overgewicht en leiden er 1 miljoen mensen aan diabetes. Dit aantal zal tot 2025 met 30\% toenemen. Kortom, deze aantallen maken duidelijk dat wetenschappelijk onderzoek naar deze ziekten van groot belang is voor mens, maatschappij, gezondheid en economie.

Diabetes, ook wel suikerziekte, is een ziekte waarbij de suikerstofwisseling verstoord is. Via de voeding krijgt ons lichaam koolhydraten binnen in de vorm van suiker, zetmeel en vezels. Deze koolhydraten worden in ons lichaam omgezet naar glucose en worden in de weefsels (o.a. in de spieren) opgenomen en omgezet in energie. Het hormoon insuline, dat aangemaakt wordt in de alvleesklier, reguleert de opname van glucose in de weefsels. Dit mechanisme is verstoord bij mensen met diabetes. Er zijn twee soorten diabetes: type 1 en type 2. Bij type 1 diabetes (T1D) is er sprake van auto-immuundestructie van de $\beta$ cellen in de alvleesklier. Hierdoor is er een absoluut tekort aan insuline met als gevolg hoge bloedsuikerwaarden. Patiënten kunnen dan insuline spuiten om de bloedsuikerwaarden te normaliseren, bijvoorbeeld na een maaltijd. Ongeveer $10 \%$ van alle patiënten met diabetes heeft T1D. Bij type 2 diabetes (T2D) is er sprake van verminderde gevoeligheid voor insuline. Daarnaast wordt er ook minder insuline aangemaakt waardoor de bloedsuikerwaarde stijgt. Negentig procent van alle patiënten met T2D wordt veroorzaakt door leefstijffactoren, zoals overgewicht en het gebrek aan fysieke activiteit. Mensen met diabetes hebben een 2 tot 4 keer hoger risico op het krijgen van hart- en vaatziekten ten opzichte van gezonde mensen. In het bijzonder, problemen aan de kleine bloedvaten (ogen, nieren en zenuwen) en aan de grote bloedvaten (hartaanval, beroerte en atherosclerose) zorgen voor dit toegenomen risico. Diabetes zorgt voor een permanente en significante vermindering van de kwaliteit van leven en voor een toename aan de kosten in de gezondheidszorg. Het is daarom van medisch en economisch belang dat diabetes en de daarmee gerelateerde hart- en vaatziekten voorkomen worden. 


\section{Diagnose \& onderzoek}

Vroege indicaties van een verhoogd risico voor hart- en vaatziekten bij patiënten met diabetes, en het toepassen van adequate interventies zal de negatieve gevolgen van deze ziekte sterk doen verlagen.

De enorme impact die hart- en vaatziekten met zich meebrengt dwingt ons tot het zoeken naar geschikte biomarkers, die voorspellend zijn voor hart- en vaatziekten in patiënten met diabetes. Echter, de traditionele cardiovasculaire risicofactoren zijn niet in staat het volledige mechanisme te verklaren. Een beter inzicht in de pathofysiologische routes en uitgebreid onderzoek naar hart- en vaatziekten bij mensen met (pre)diabetes is daarom noodzakelijk.

\section{Advanced glycation endproducts (AGE's)}

Versuikering van eiwitten, ook wel glycering genoemd, werd voor het eerst beschreven door de Franse arts en wetenschapper Louis Camille Maillard in 1912. Hij onderzocht de reactie tussen aminozuren en suiker en ontdekte dat deze reactie verantwoordelijk was voor de bruine kleur, specifieke smaak en geur bij de bereiding van voedsel. De naar hem vernoemde $M$ aillard reactie is niet één simpele reactie tussen een aminozuur en suiker, maar een cascade van complexe reacties met uiteindelijk de vorming van advanced glycation endproducts (AGE's).

De vorming van deze AGE's vindt niet alleen plaats bij de bereiding van voedsel maar ook in het menselijk lichaam. Voornamelijk bij patiënten met diabetes, als gevolg van hoge bloedsuikerwaarden, maar ook als gevolg van een verminderde klaring, bij patiënten met chronische nierziekte. AGE's zijn in verband gebracht met diabetische complicaties en hart- en vaatziekten.

In het verleden werden immunochemische analyse technieken (met name enzyme-linked immunosorbent assays, ELISA's) gebruikt voor het kwantificeren van AGE's. Echter, de kwaliteit en betrouwbaarheid van deze methoden lieten te wensen over. Er moest dus gezocht worden naar gevoeligere en specifiekere technieken. Vloeistofchromatografie was een analysetechniek die al jaren succesvol werd toegepast voor het kwantificeren van biomoleculen. Deze techniek bleek ook uitermate geschikt te zijn voor de analyse van AGE's en andere aanverwante componenten. In dit proefschrift wordt een overzicht gegeven van de ontwikkeling en validatie van een aantal nieuwe chromatografische technieken alsook de toepassing van deze technieken in klinische studies voor de detectie van AGE's en zijn voorlopers. 


\section{Belangrijkste bevindingen uit dit proefschrift}

In hoofdstuk 2A wordt een snelle en gevoelige vloeistofchromatografische methode beschreven voor het kwantificeren van pentosidine met fluorescentie als detectiemethode. De belangrijkste verbeteringen van deze nieuwe methode ten opzichte van de al beschreven methoden zijn: kortere runtijden, het gebruik van "reversed-phase" chromatografie in plaats van ingewikkelde chromatografische systemen en een eenvoudige monstervoorbewerking. De beschreven methode werd succesvol toegepast voor het kwantificeren van eiwitgebonden pentosidine in plasma van gezonde vrijwilligers en patiënten met nierfalen. Er werd een significant hogere plasma pentosidine concentratie gevonden in de patiënten met nierfalen ten opzichte van gezonde vrijwilligers. Deze resultaten waren in overeenstemming met de literatuur.

Hoge druk vloeistofchromatografie gekoppeld aan massaspectrometrie is een analysetechniek die zijn waarde al in vele wetenschappelijke publicaties heeft bewezen. Voornamelijk de introductie en gebruik van zeer kleine partikels $(<1.7 \mu \mathrm{m})$ bij het pakken van chromatografische kolommen heeft voor een enorme verbetering van de chromatografie gezorgd. De koppeling van deze scheidings-techniek (ultra performace liquid chromatography, UPLC) aan een zeer gevoelige en selectieve massaspectromter (tandem MS) resulteert in een van de beste analysetechnieken van dit moment.

In hoofdstuk 2B wordt deze techniek toegepast voor de analyse van drie eiwitgebonden en vrije $A G E^{\prime} s$ in plasma, te weten: $N^{\varepsilon}$-(carboxymethyl)lysine $(C M L), \quad N^{\varepsilon}-(1-$ carboxyethyl)lysine (CEL) en $\mathrm{N}^{\delta}$-(5-hydro-5-methyl-4-imidazolon-2-yl)-ornithine (M G-H1). Daarnaast was het met dit systeem ook mogelijk het aminozuur lysine te analyseren, dat gebruikt werd als een maat voor de hoeveelheid eiwit. Met deze techniek werd plasma onderzocht van gezonde vrijwilligers en patiënten met nierfalen. Zowel vrij- als eiwitgebonden plasma concentraties van CML, CEL en MG-H1 waren hoger in de patiënten met nierfalen ten opzichte van de gezonde vrijwilligers.

In hoofdstuk 3 werden de technieken, zoals beschreven in hoofdstuk $2 \mathrm{~A}$ en $2 \mathrm{~B}$, gebruikt voor het onderzoeken van de samenhang tussen plasma AGE's en hart- en vaatziekten, in individuen met verschillende gradaties aan glucosetolerantie. Hiervoor werden eiwitgebonden plasma concentraties van CML, CEL en pentosidine gemeten, in twee Nederlandse cohort studies. Verassend genoeg werden er geen verbanden gevonden tussen de eiwitgebonden AGE's en hart- en vaatziekten. Daarnaast bleken eiwitgebonden plasma concentraties van CM L, CEL en pentosidine, niet hoger te zijn in mensen met type 2 diabetes. Dit resultaat was in tegenstelling tot de eerder gepubliceerde data in mensen met type 1 diabetes.

Het derivatiseren van biomoleculen wordt vaak gebruikt om de polariteit te veranderen of het molecuul gevoeliger en toepasbaar te maken voor bepaalde detectietechnieken. In hoofdstuk 4 werd een chirale derivatisering, met diacetyl-L-wijsteenzuuranhydride, toegepast voor het scheiden van L- en D-lactaat met behulp van reversed-phase (RP) 
chromatografie gekoppeld aan tandem MS. We waren voornamelijk geïnteresseerd in Dlactaat omdat dit het eindproduct is van het glyoxalase systeem, dat verantwoordelijk is voor de detoxificering van methylglyoxal. Met behulp van deze methode werden concentraties van L- en D-lactaat gekwantificeerd in plasma en urine van gezonde vrijwilligers, patiënten met inflammatoire darmziekten en patiënten met T2D. Plasma en urine L- en D-lactaat concentraties waren significant hoger in patiënten met T2D. In patiënten met inflammatoire darmziekten werd alleen een significant hogere concentratie aan urine L-lactaat gevonden ten opzichte van gezonde vrijwilligers. Deze resultaten bevestigen dat $D$-lactaat een reflectie is van de detoxificatie van methylglyoxaal door het glyoxalase systeem en niet van de D-lactaat productie in de darmen.

In hoofdstuk 5 werd de samenhang van plasma D-lactaat concentraties met insuline resistentie onderzocht. Hiervoor werd cross-sectioneel onderzoek gedaan in het CODAM cohort (cohort study on diabetes and atherosclerosis M aastricht). Er werd een positieve associatie gevonden tussen plasma D-lactaat en insuline resistentie, onafhankelijk van potentiële confounders. Deze resultaten suggereren dat methylglyoxal een rol speelt in insuline resistentie. Echter, om dit te bevestigen zal verder onderzoek noodzakelijk zijn met specifieke interventies.

In hoofdstuk 4 en 5 werd reeds gespeculeerd over de mogelijke rol van methylglyoxaal bij het ontwikkelen van insuline resistentie. Om deze resultaten te bevestigen en verder te onderzoeken werd in hoofdstuk 6 een nieuwe en verbeterde methode opgezet voor het analyseren van drie $\alpha$-oxoaldehydes, te weten: glyoxaal, methylglyoxaal en 3deoxyglucosone. Daarnaast werd een uitgebreide validatie van de methode en monstervoorbewerking uitgevoerd. Om stabiele en betrouwbare concentraties van deze $\alpha$-oxoaldehydes te verkrijgen vonden we dat het bloed afgenomen dient te worden in EDTA buizen en een directe onteiwiting met perchloorzuur noodzakelijk is om de $\alpha$ oxoaldehyde concentraties te stabiliseren. Met de beschreven methode werden verhoogde plasma $\alpha$-oxoaldehyde concentraties gevonden in patiënten met T2D ten opzichte van gezonde controles.

In hoofdstuk $\mathbf{7}$ werd de invloed van een orale glucose belasting op plasma $\alpha$-oxoaldehyde concentraties onderzocht. Hiervoor werd cross-sectioneel onderzoek gedaan in het CODAM cohort. Plasma concentraties van glyoxaal, methylglyoxaal en 3-deoxyglucosone werden gemeten in de nuchtere toestand en na een orale glucose tolerantie test (OGT). Het hebben van prediabetes en type 2 diabetes was geassocieerd met hogere plasma $\alpha$ oxoaldehyde concentraties gedurende de OGTT. Deze resultaten tonen aan dat er een stijging is van dicarbonyl stress in voornamelijk patiënten met (pre)diabetes na het nuttigen van glusose-houdende dranken.

Deze verhoogde productie heeft mogelijk ook consequenties voor de vorming van AGE's. Echter, de endogene vorming van AGE's is veel minder snel dan de vorming van $\alpha$ oxoaldehydes en zal daardoor waarschijnlijk geen acuut effect laten zien. 
In de inleiding werd beschreven dat AGE's aanwezig zijn in voedsel, voornamelijk bij de bereiding (bakken, grillen en frituren) van voedsel worden deze AGE's gevormd. M ogelijk leveren deze in voeding aanwezige AGE's, wel een directe bijdrage op de concentratie van circulerende AGE's in plasma. Om dit te onderzoeken dient men inzicht te krijgen in de hoeveelheid AGE's per voedingsproduct. In hoofdstuk 8 werd daarom een UPLC-tandem MS methode ontwikkeld voor het analyseren van AGE's in voedingsproducten geconsumeerd in een westers dieet. Eiwitgebonden CML, CEL en MG-H1 concentraties werden in 190 verschillende voedingsproducten gekwantificeerd. Fruit, groente, boter en koffie waren producten met een lage of verwaarloosbare AGE concentratie. Hoge AGE concentraties werden gevonden in gebakken, gefrituurde en gegrilde producten, zoals vlees, pindakaas, frikadel en noten.

In hoofdstuk 9 werd de voedings-AGE-database, zoals beschreven in hoofdstuk 8, gebruikt om de associatie tussen ingenomen AGE's (via de voeding) en circulerende AGE's te onderzoeken. Plasma en urine concentraties van $\mathrm{CML}$, CEL en MG-H1 werden geanalyseerd in 465 individuen van het CODAM cohort. Daarnaast werd de AGE-inname geschat aan de hand van een voedingsvragenlijst en de AGE-database. De conclusie van dit onderzoek was, dat het eten van voedsel rijk aan AGE's geassocieerd is met de concentraties van vrije AGE's, zoals gemeten in plasma en urine. Deze resultaten duiden op de mogelijke consequenties van het eten van een dieet rijk aan AGE's.

\section{Conclusie}

Dit proefschrift beschrijft de toepassing van nieuwe analysetechnieken voor het onderzoeken van AGE's en zijn voorlopers in diverse matrices. De belangrijkste conclusie van dit proefschrift is dat vloeistofchromatografie gekoppeld aan massaspectrometrie een waardevolle en zeer betrouwbare methode is voor het analyseren van biomoleculen in complexe matrices. M et deze techniek werd aangetoond dat AGE's en zijn voorlopers nauw verband houden met verschillende ziekten, zoals diabetes, nierziekte, hart- en vaatziekten en inflammatoire darmziekte. Echter, het causale verband tussen AGE's en deze ziekten zal nog verder onderzocht moeten worden. Hiervoor zullen specifieke interventiestudies noodzakelijk zijn, waarbij gekeken wordt naar het remmen van AGE's of verlaging van AGE's vanuit de voeding en de invloed hiervan op bijvoorbeeld insuline resistentie of het ontwikkelen van hart- en vaatziekten. De toepassing van de technieken, beschreven in dit proefschrift, zullen een noodzakelijke rol spelen in dit vervolgonderzoek. Daarnaast staan de ontwikkeling van nieuwe technieken voor het analyseren van andere AGE's en biomoleculen niet stil. De zoektocht naar geschikte biomarkers, voor het voorspellen van ziekten en complicaties en daarmee de behandelingsstrategie te verbeteren, zal de inzet zijn van toekomstig onderzoek. 

Valorization 



\section{Valorization}

More than 2000 years ago, Hippocrates, a well-known Greek physician, was one of the founding fathers of the modern pathology. He developed new methods to examine the human body and was one of the first who used body fluids (blood, phlegm, yellow bile and black bile) in order to understand the etiology and pathogenesis of a number of diseases. Today, researchers are still using body fluids (such as blood, plasma, serum, feces, saliva and urine) to investigate and understand diseases. One way of doing these investigations, is using measureable indicators to examine the physiological state of an individual. These indicators, also known as (disease-related) biomarkers, are a valuable tool to understand the mechanisms, and even more important, treat and prevent many diseases. Sensitive, specific and reliable state-of-the-art techniques are necessary to determine these biomarkers in many different matrices. In the present thesis we validated and developed new analytical methods to quantify specific biomarkers in order to investigate diabetes and cardiovascular disease (CVD).

\section{Relevance of this thesis}

In 2012, an estimated 1.5 million deaths were directly caused by diabetes ${ }^{1}$ and even 17.5 million people died from CVD ${ }^{2}$. Individuals with diabetes are at an increased risk of cardiovascular events ${ }^{3,4}$ and its associated complications leads to a permanent and significant loss of quality of life. Therefore, prevention of diabetes and in case of diabetes, prevention of cardiovascular disease, is of highest importance and clearly represents a medical and economical need ${ }^{5}$. Future strategies should therefore focus primarily on the prevention of diabetes. Given the enormous impact of cardiovascular disease, it is of utmost importance to find biomarkers for the identification of patients with diabetes which are at high risk of developing vascular complications and morbidity; i.e. to improve risk prediction ${ }^{6}$. In this thesis we focused on the analysis of advanced glycation endproducts (AGEs) and related biomarkers in relation to diabetes and CVD.

\section{Advanced glycation endproducts and disease}

AGEs are formed by the reaction of proteins with reactive metabolic intermediates derived from glucose and from lipid oxidation with the involvement of oxidative stress ${ }^{7-9}$. Increased endogenous formation and accumulation of AGEs is common in patients with diabetes, and, because of impaired clearance, in those with chronic kidney disease $(C K D)^{10}$ or end stage renal disease (ESRD $)^{11}$. AGEs are implicated to play a role in the development of vascular disease ${ }^{12-17}$, age-related disease ${ }^{13,18,19}$ and diabetes ${ }^{20}$. Most recent, it has become apparent that dietary AGEs represent a significant source of circulating and tissue AGEs ${ }^{21-25}$. 
However, many of these AGE-related researches were based on techniques, such as enzyme-linked immunosorbent assay (ELISA). However, new and improved analytical techniques are necessary in order to investigate the role of these AGEs in disease.

\section{Applications of biomarker analysis}

In this thesis we have used ultra-performance liquid chromatography tandem mass spectrometry (UPLC-M S/MS) to determine AGEs and other related biomarkers, such as methylglyoxal, in healthy individuals, patients with type 2 diabetes mellitus (T2DM), patients with impaired glucose metabolism (IGM), patients with inflammatory bowel disease (IBD) and patients with end stage renal disease (ESRD). M oreover, this technique was used to determine the AGE content in foods. AGEs have traditionally been detected by ELISA ${ }^{26-32}$. For several reasons the use of antisera for quantitative immunoassays of protein-bound AGEs is questionable. Reproducibility and sensitivity of such an assay are not optimal, because the specificity of the antibodies is often difficult to define and, because of steric constraints, not all AGE epitopes on the protein may be available for interaction with the antibody ${ }^{28,30}$. Thus, AGE measurements with immunoassays should be interpreted with care. A better approach for the quantitative determination of specific AGEs is the use of a specific analytical technique. For the determination of AGE levels in both tissue and blood samples, high performance liquid chromatography (HPLC) measurements $\mathrm{s}^{33-36}$ and several mass spectrometry methods have been developed including gas chromatography mass spectrometry (GC-MS) $)^{37,38}$, and liquid chromatography tandem mass spectrometry (LC-MS/MS) ${ }^{32,36,39,40}$. However, the simultaneous quantification of different AGEs in plasma or tissue, using a highly sensitive, selective and rapid analytical method, has not been reported yet.

In chapter 2, 4, 6 and 8 state-of-the-art techniques were described to analyze pentosidine, $\mathrm{N}^{\varepsilon}$-(carboxymethyl)/ysine (CML), $\mathrm{N}^{\varepsilon}$-(carboxyethyl)/ysine (CEL), 5-hydro-5methylimidazolone (MG-H1), L-lactate, D-lactate, methylglyoxal, glyoxal and 3-deoxyglucosone. These techniques were successfully applied to investigate disease mechanisms.

In chapter $\mathbf{5}$ and $\mathbf{7}$ we describe the potential role of the biomarkers D-lactate and methylglyoxal in insulin resistance and the increased risk of diabetic complications. We found increased plasma concentrations of $\alpha$-dicarbonyls and D-lactate in patients with T2DM as compared to non-diabetic controls. Moreover, an oral glucose tolerance test (OGTT) was performed in individuals with normal glucose metabolism (NGM), IGM and T2DM. The area under the curve (AUC) for the OGTT levels of the $\alpha$-dicarbonyls and glucose were higher in individuals with IGM and T2DM as compared with NGM. 
These findings underline the potential importance of $\alpha$-dicarbonyl stress as a candidate to explain the increased risk of diabetic complications in individuals with postprandial hyperglycemia.

In chapter 8 we validated a new method to determine dietary AGES (CML, CEL and MG-H1) in 190 food products as consumed in a Western diet. A dietary AGE database was presented and applied to calculate the dietary AGE intake of a Dutch cohort. In chapter 9 we used these data and demonstrated that higher intake of dietary AGEs is associated with higher levels of plasma and urinary AGEs. These findings are an important step in understanding the metabolic transit of these dietary AGEs. Several studies indicate that dietary AGEs are associated with poor health ${ }^{13-15,23,29,42}$. Future prospective studies should address whether dietary AGEs are associated with adverse outcomes such as accelerated development of cardiovascular disease, diabetes and other age-related diseases.

\section{Future perspective}

In the following years the UPLC-MS/MS applications, described in this thesis, will be used in many different studies. They create opportunities to investigate not only the glycation pathway but also other disease mechanisms in more detail. However, as new research questions arise, analytical methods will need to evolve as well. In this thesis we have made the first step in investigating the complex biochemical pathway of glycation and related mechanisms. We have shown that glucose is the key molecule which is responsible for initiating glycation and formation of reactive $\alpha$-dicarbonyls. We have also shown that dietary AGEs are influencing circulating AGEs. However, several questions need to be answered: 1. What is the effect of AGEs for human health? and 2. Do dietary AGEs play a significant role in developing diseases, such as diabetes and CVD? To answer these questions, the effect of AGE inhibitors and AGE-diet interventions need to be investigated. These studies are ongoing. Biomolecule analysis with state-of-the-art UPLCMS/M Stechniques, such as the one described int this thesis, will be a fundamental part in these studies. 


\section{References}

WHO. Diabetes: Fact sheet no. 312, (2015).

WHO. Diabetes: Fact sheet no. 317, (2016).

Sarwar, N. et al. Diabetes mellitus, fasting blood glucose concentration, and risk of vascular disease: a collaborative meta-analysis of 102 prospective studies. Lancet 375, 2215-2222, doi:10.1016/s01406736(10)60484-9 (2010).

Fox, C. S. et al. Trends in cardiovascular complications of diabetes. Jama 292, 2495-2499, doi:10.1001/jama.292.20.2495 (2004).

da Rocha Fernandes, J. et al. IDF Diabetes Atlas estimates of 2014 global health expenditures on diabetes. Diabetes Res Clin Pract 117, 48-54, doi:10.1016/j.diabres.2016.04.016 (2016).

Herder, C., Karakas, M. \& Koenig, W. Biomarkers for the prediction of type 2 diabetes and cardiovascular disease. Clinical pharmacology and therapeutics 90, 52-66, doi:10.1038/clpt.2011.93 (2011).

Brownlee, M. Advanced protein glycosylation in diabetes and aging. Annual review of medicine 46, 223-234, doi:10.1146/annurev.med.46.1.223 (1995).

Brownlee, M., Vlassara, H. \& Cerami, A. Nonenzymatic glycosylation and the pathogenesis of diabetic complications. Annals of internal medicine 101, 527-537 (1984).

Monnier, V. M. Nonenzymatic glycosylation, the Maillard reaction and the aging process. Journal of gerontology 45, B105-111 (1990).

Stinghen, A. E., Massy, Z. A., Vlassara, H., Striker, G. E. \& Boullier, A. Uremic Toxicity of Advanced Glycation End Products in CKD. J Am Soc Nephrol 27, 354-370, doi:10.1681/asn.2014101047 (2016).

Suliman, M. E. et al. Plasma pentosidine is associated with inflammation and malnutrition in endstage renal disease patients starting on dialysis therapy. J Am Soc Nephrol 14, 1614-1622 (2003).

Baumann, M. et al. N epsilon-(carboxymethyl)lysine during the early development of hypertension. Ann N Y Acad Sci 1126, 201-204 (2008).

3 Hanssen, N. M. et al. Higher levels of advanced glycation endproducts in human carotid atherosclerotic plaques are associated with a rupture-prone phenotype. Eur Heart J 35, 1137-1146, doi:10.1093/ eurheartj/eht402 (2014).

4 Hartog, J. W. et al. Clinical and prognostic value of advanced glycation end-products in chronic heart failure. Eur Heart J 28, 2879-2885 (2007).

5 Lieuw, A. F. M. L. et al. Increased levels of $\mathrm{N}$ (epsilon)-(carboxymethyl)lysine and $\mathrm{N}$ (epsilon)(carboxyethyl)lysine in type 1 diabetic patients with impaired renal function: correlation with markers of endothelial dysfunction. Nephrol Dial Transplant 19, 631-636 (2004).

$6 \mathrm{Nin}, \mathrm{J}$. W. et al. Higher plasma levels of advanced glycation end products are associated with incident cardiovascular disease and all-cause mortality in type 1 diabetes: a 12-year follow-up study. Diabetes Care 34, 442-447, doi:10.2337/dc10-1087 (2011).

Verzijl, N. et al. Crosslinking by advanced glycation end products increases the stiffness of the collagen network in human articular cartilage: a possible mechanism through which age is a risk factor for osteoarthritis. Arthritis and rheumatism 46, 114-123, doi:10.1002/15290131(200201)46:1<114::aid-art10025>3.0.c0;2-p (2002). 
Strozecki, P. et al. Advanced glycation end products and arterial stiffness in patients with diabetic nephropathy and patients with chronic kidney disease without diabetes. Polskie Archiwum M edycyny Wewnetrznej 123, 609-616 (2013).

Sourris, K. C. et al. Plasma advanced glycation end products (AGEs) and NF-kappaB activity are independent determinants of diastolic and pulse pressure. Clin Chem Lab Med 52, 129-138, doi:10.1515/cclm-2012-0850 (2014).

Teichert, T. et al. Association between Advanced Glycation End Products and Impaired Fasting Glucose: Results from the SALIA Study. PLoS One 10, e0128293, doi:10.1371/journal.pone.0128293 (2015).

Clarke, R. E., Dordevic, A. L., Tan, S. M., Ryan, L. \& Coughlan, M. T. Dietary Advanced Glycation End Products and Risk Factors for Chronic Disease: A Systematic Review of Randomised Controlled Trials. Nutrients 8, doi:10.3390/nu8030125 (2016). Uribarri, J. et al. Dietary advanced glycation end products and their role in health and disease. Advances in nutrition 6, 461-473, doi:10.3945/an.115.008433 (2015).

Stirban, A. \& Tschope, D. Vascular Effects of Dietary Advanced Glycation End Products. International journal of endocrinology 2015, 836498, doi:10.1155/2015/836498 (2015). de Courten, B. et al. Diet low in advanced glycation end products increases insulin sensitivity in healthy overweight individuals: a double-blind, randomized, crossover trial. Am J Clin Nutr, doi:10.3945/ajcn.115.125427 (2016).

Angoorani, P., Ejtahed, H. S., M irmiran, P., M irzaei, S. \& Azizi, F. Dietary consumption of advanced glycation end products and risk of metabolic syndrome. International journal of food sciences and nutrition 67, 170-176, doi:10.3109/09637486.2015.1137889 (2016).

Sanaka, T. et al. Plasma pentosidine levels measured by a newly developed method using ELISA in patients with chronic renal failure. Nephron 91, 64-73 (2002).

Izuhara, Y. et al. A sensitive and specific ELISA for plasma pentosidine. Nephrol Dial Transplant 14, 576-580 (1999).

M itsuhashi, T., Vlassara, H., Founds, H. W. \& Li, Y. M. Standardizing the immunological measurement of advanced glycation endproducts using normal human serum. J Immunol Methods 207, 79-88 (1997).

Munch, G. et al. Determination of advanced glycation end products in serum by fluorescence spectroscopy and competitive ELISA. European journal of clinical chemistry and clinical biochemistry : journal of the Forum of European Clinical Chemistry Societies 35, 669-677 (1997).

Dorrian, C. A., Cathcart, S., Clausen, J., Shapiro, D. \& Dominiczak, M. H. Factors in human serum interfere with the measurement of advanced glycation endproducts. Cellular and molecular biology 44, 1069-1079 (1998).

Takayama, F. et al. Immunohistochemical detection of imidazolone and N(epsilon)(carboxymethyl)lysine in aortas of hemodialysis patients. Cellular and molecular biology 44, 11011109 (1998).

32 Srey, C. et al. Immunochemical and mass spectrometric analysis of Nepsilon-(carboxymethyl)lysine content of AGE-BSA systems prepared with and without selected antiglycation agents. Journal of agricultural and food chemistry 58, 11955-11961, doi:10.1021/jf102543g (2010). 

endproducts (AGEs): surveying AGEs by chromatographic assay with derivatization by 6aminoquinolyl-N-hydroxysuccinimidyl-carbamate and application to Nepsilon-carboxymethyl-lysineand Nepsilon-(1-carboxyethyl)lysine-modified albumin. Biochem J 364, 1-14 (2002). Ahmed, N. \& Thornalley, P. J. Chromatographic assay of glycation adducts in human serum albumin glycated in vitro by derivatization with 6-aminoquinolyl- $\mathrm{N}$-hydroxysuccinimidyl-carbamate and intrinsic fluorescence. Biochem J 364, 15-24 (2002). Hartkopf, J. \& Pahlke, C. Determination of N-carboxymethyllysine by a reversed-phase highperformance liquid chromatography method. J Chromatogr A 672, 242-246 (1994). Ahmed, M. U., Thorpe, S. R. \& Baynes, J. W. Identification of N epsilon-carboxymethyllysine as a degradation product of fructoselysine in glycated protein. J Biol Chem 261, 4889-4894 (1986). Charissou, A., Ait-Ameur, L. \& Birlouez-Aragon, I. Evaluation of a gas chromatography/mass spectrometry method for the quantification of carboxymethyllysine in food samples. Journal of chromatography. A 1140, 189-194, doi:10.1016/j.chroma.2006.11.066 (2007). Anderson, M. M. \& Heinecke, J. W. Production of N(epsilon)-(carboxymethyl)lysine is impaired in mice deficient in NADPH oxidase: a role for phagocyte-derived oxidants in the formation of advanced glycation end products during inflammation. Diabetes 52, 2137-2143 (2003).

39 Teerlink, T., Barto, R., Ten Brink, H. J. \& Schalkwijk, C. G. Measurement of Nepsilon(carboxymethyl)lysine and Nepsilon-(carboxyethyl)lysine in human plasma protein by stable-isotopedilution tandem mass spectrometry. Clin Chem 50, 1222-1228 (2004).

40 Thornalley, P. J. et al. Quantitative screening of advanced glycation endproducts in cellular and extracellular proteins by tandem mass spectrometry. Biochem J 375, 581-592 (2003). 


\section{Curriculum vitae}





\section{Curriculum vitae}

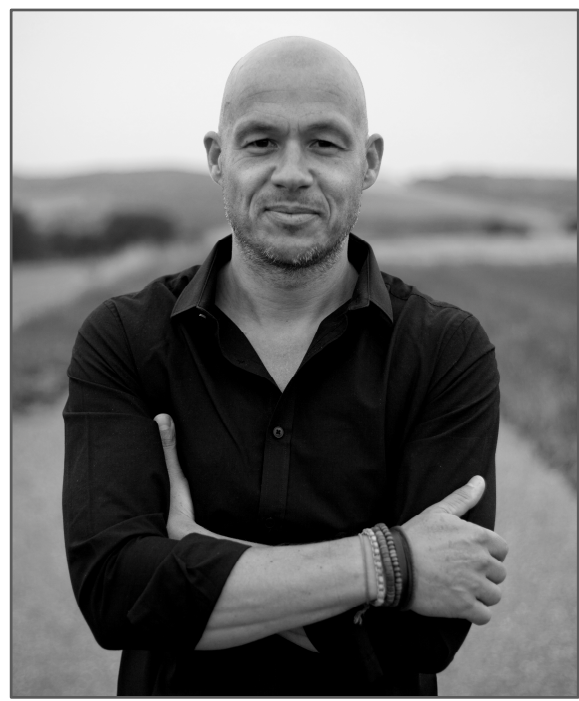

Jean Scheijen was born on August $29^{\text {th }}, 1971$ in Heerlen and raised in Schin op Geul (the Netherlands). He graduated from secondary school (Ravensberg College, Valkenburg aan de Geul) in 1987 and started in that same year his study Analytical Chemistry at the Walram College in Sittard. During this period he did an internship at the Laboratory of Rolith Chemicals (Roermond) where he was responsible for the quality control of the production line.

After graduation in 1992, he started studying Environmental Chemistry at the Hogeschool Limburg in Sittard and graduated in 1997. During this period he did an internship and master project at the Laboratory of Clinical Pharmacology and Toxicology (M aastricht University M edical Centre, M UM C+).

Hereafter, he started working at the Laboratory of Inborn Errors of Metabolism (1998, Clinical Genetics, MUM C+). After one year he was offered a job at the Department of General Surgery (Maastricht University) and obtained here his first experience with Liquid Chromatography Mass Spectrometry (LC-MS). For five years he worked under the supervision of M ick Deutz and Hans van Eijk at this department.

In 2003, the department of Clinical Genetics wanted to implement new analytical techniques to investigate inborn errors. Together with Huub Waterval, Jean Scheijen implemented two LC-MS systems at the Laboratory of Inborn Errors and succeeded in developing new methods to analyze several biomolecules to investigate metabolic disorders. These methods are used until this day.

After five years, he was asked to set up an analytical unit at the Laboratory of M etabolism and Vascular Medicine (Internal Medicine, Maastricht University, MUMC+). Under the supervision of Casper Schalkwijk and Coen Stehouwer he implemented several LC and LCMS methods to investigate diabetes and cardiovascular disease. In 2013, he started his $\mathrm{PhD}$ at that same department and continued working there as a Senior Research Technician. 

Scientific output 



\section{Scientific output}

\section{List of publications}

1. Skovgaard D, Svensson RB, Scheijen J, Eliasson P, M ogensen P, Hag AM, Kjaer M, Schalkwijk CG, Schjerling P, M agnusson SP, Couppe C. An advanced glycation endproduct (age)-rich diet promotes accumulation of ages in achilles tendon. Physiological reports. 2017; 5(6). pii: e13215

2. Scheijen 니M, Hanssen NMJ, Greevenbroek MM, Kallen CJ, Feskens EJM, Stehouwer CDA, Schalkwijk CG. Dietary intake of advanced glycation endproducts is associated with higher levels of advanced glycation endproducts in plasma and urine: The codam study. Clinical Nutrition. 2017:1-7

3. Fokkens BT, Mulder DJ, Schalkwijk CG, Scheijen JL, Smit AJ, Los LI. Vitreous advanced glycation endproducts and alpha-dicarbonyls in retinal detachment patients with type 2 diabetes mellitus and non-diabetic controls. PloS one. 2017;12:e0173379

4. van Eupen MG, Schram MT, van Sloten TT, Scheijen J, Sep SJ, van der Kallen CJ, Dagnelie PC, Koster A, Schaper N, Henry RM, Kroon AA, Smit AJ, Stehouwer CD, Schalkwijk CG. Skin autofluorescence and pentosidine are associated with aortic stiffening: The maastricht study. Hypertension (Dallas, Tex. : 1979). 2016;68:956-963

5. Scheijen لL, Clevers E, Engelen L, Dagnelie PC, Brouns F, Stehouwer CD, Schalkwijk CG. Analysis of advanced glycation endproducts in selected food items by ultra-performance liquid chromatography tandem mass spectrometry: Presentation of a dietary age database. Food chemistry. 2016;190:1145-1150

6. Nokin MJ, Durieux F, Peixoto P, Chiavarina B, Peulen O, Blomme A, Turtoi A, Costanza B, Smargiasso N, Baiwir D, Scheijen JL, Schalkwijk CG, Leenders J, De Tullio P, Bianchi E, Thiry M, Uchida K, Spiegel DA, Cochrane JR, Hutton CA, De Pauw E, Delvenne P, Belpomme D, Castronovo V, Bellahcene A. Methylglyoxal, a glycolysis side-product, induces hsp90 glycation and yap-mediated tumor growth and metastasis. elife. 2016;5. pii: e19375

7. Maessen DE, Hanssen NM, Lips MA, Scheijen JL, Willems van Dijk K, Pijl H, Stehouwer CD, Schalkwijk CG. Energy restriction and roux-en-y gastric bypass reduce postprandial alpha-dicarbonyl stress in obese women with type 2 diabetes. Diabetologia. 2016;59:2013-2017 
8. Fokkens BT, M ulder DJ, Nugteren M B, Schalkwijk CG, Scheijen JL, Smit AJ, Los LI. Surface area of detachment, proliferative vitreoretinopathy, and pulse pressure, but not ages, are associated with retinal redetachment. Investigative ophthalmology $\&$ visual science. 2016;57:6633-6638

9. de Courten B, de Courten MP, Soldatos G, Dougherty SL, Straznicky N, Schlaich $M$, Sourris KC, Chand V, Scheijen JL, Kingwell BA, Cooper ME, Schalkwijk CG, Walker KZ, Forbes JM. Diet low in advanced glycation end products increases insulin sensitivity in healthy overweight individuals: A double-blind, randomized, crossover trial. The American journal of clinical nutrition. 2016;103:1426-1433

10. Maessen DE, Hanssen NM, Scheijen JL, van der Kallen CJ, van Greevenbroek M M , Stehouwer CD, Schalkwijk CG. Post-glucose load plasma alpha-dicarbonyl concentrations are increased in individuals with impaired glucose metabolism and type 2 diabetes: The codam study. Diabetes care. 2015;38:913-920

11. Hanssen NM, Beulens JW, van Dieren S, Scheijen JL, van der AD, Spijkerman AM, van der Schouw YT, Stehouwer CD, Schalkwijk CG. Plasma advanced glycation end products are associated with incident cardiovascular events in individuals with type 2 diabetes: A case-cohort study with a median follow-up of 10 years (epic-nl). Diabetes. 2015;64:257-265

12. Engelen L, Schalkwijk CG, Eussen SJ, Scheijen JL, Soedamah-Muthu SS, Chaturvedi N, Fuller JH, Stehouwer CD. Low 25-hydroxyvitamin d2 and 25-hydroxyvitamin d3 levels are independently associated with macroalbuminuria, but not with retinopathy and macrovascular disease in type 1 diabetes: The eurodiab prospective complications study. Cardiovascular diabetology. 2015;14:67

13. Cornelis T, Eloot S, Vanholder R, Glorieux G, van der Sande FM, Scheijen JL, Leunissen KM, Kooman JP, Schalkwijk CG. Protein-bound uraemic toxins, dicarbonyl stress and advanced glycation end products in conventional and extended haemodialysis and haemodiafiltration. Nephrology, dialysis, transplantation : official publication of the European Dialysis and Transplant Association - European Renal Association. 2015;30:1395-1402

14. Scheijen JL, Schalkwijk CG. Quantification of glyoxal, methylglyoxal and 3deoxyglucosone in blood and plasma by ultra performance liquid chromatography tandem mass spectrometry: Evaluation of blood specimen. Clinical chemistry and laboratory medicine. 2014;52:85-91 
15. Hanssen NM, Wouters K, Huijberts MS, Gijbels MJ, Sluimer JC, Scheijen JL, Heeneman S, Biessen EA, Daemen MJ, Brownlee M, de Kleijn DP, Stehouwer CD, Pasterkamp G, Schalkwijk CG. Higher levels of advanced glycation endproducts in human carotid atherosclerotic plaques are associated with a rupture-prone phenotype. European heart journal. 2014;35:1137-1146

16. Gopal P, Reynaert NL, Scheijen JL, Schalkwijk CG, Franssen FM, Wouters EF, Rutten EP. Association of plasma srage, but not esrage with lung function impairment in copd. Respiratory research. 2014;15:24

17. Gopal P, Reynaert NL, Scheijen JL, Engelen L, Schalkwijk CG, Franssen FM, Wouters EF, Rutten EP. Plasma advanced glycation end-products and skin autofluorescence are increased in copd. The European respiratory journal. 2014;43:430438

18. Eriksen C, Svensson RB, Scheijen J, Hag AM, Schalkwijk C, Praet SF, Schjerling P, Kjaer M, Magnusson SP, Couppe $C$. Systemic stiffening of mouse tail tendon is related to dietary advanced glycation end products but not high-fat diet or cholesterol. Journal of applied physiology (Bethesda, M d. : 1985). 2014;117:840-847

19. van Eupen MG, Schram MT, Colhoun HM, Scheijen JL, Stehouwer CD, Schalkwijk CG. Plasma levels of advanced glycation endproducts are associated with type 1 diabetes and coronary artery calcification. Cardiovascular diabetology. 2013;12:149

20. Hanssen NM, Engelen L, Ferreira I, Scheijen JL, Huijberts MS, van Greevenbroek M M, van der Kallen CJ, Dekker JM, Nijpels G, Stehouwer CD, Schalkwijk CG. Plasma levels of advanced glycation endproducts nepsilon-(carboxymethyl)lysine, nepsilon(carboxyethyl)lysine, and pentosidine are not independently associated with cardiovascular disease in individuals with or without type 2 diabetes: The hoorn and codam studies. The Journal of clinical endocrinology and metabolism. 2013;98:E13691373

21. Scheijen $\mathbf{J}$, Hanssen NM, van de Waarenburg M P, Jonkers DM, Stehouwer CD, Schalkwijk CG. $L(+)$ and $d(-)$ lactate are increased in plasma and urine samples of type 2 diabetes as measured by a simultaneous quantification of $\mathrm{I}(+)$ and $\mathrm{d}(-)$ lactate by reversed-phase liquid chromatography tandem mass spectrometry. Experimental diabetes research. 2012;2012:234812 
22. Alkhalaf A, Kleefstra N, Groenier KH, Bilo HJ, Gans RO, Heeringa P, Scheijen JL, Schalkwijk CG, Navis GJ, Bakker SJ. Effect of benfotiamine on advanced glycation endproducts and markers of endothelial dysfunction and inflammation in diabetic nephropathy. PloS one. 2012;7:e40427

23. Cnossen TT, Gladziwa U, van de Kerkhof JJ, Schalkwijk CG, Scheijen J, van Amersfoort J, Moret K, Beerenhout CH, Kooman JP. The influence of bicarbonate/lactatebuffered pd fluids on $\mathrm{n}$ \{epsilon\}(carboxyethyl)lysine and $\mathrm{n}$ \{epsilon\} (carboxymethyl)lysine in peritoneal effluent. Peritoneal dialysis international : journal of the International Society for Peritoneal Dialysis. 2011;31:189-193

24. Scheijen $\mathbf{L}$, Waterval WA, Ortmans-Ploemen MM, Habets-van der Poel CD, Bierau J. Quantitative uplc-ms/ms analysis of underivatised amino acids in body fluids is a reliable tool for the diagnosis and follow-up of patients with inborn errors of metabolism. Clinica chimica acta; international journal of clinical chemistry. 2009;407:36-42

25. Scheijen $\mathbf{L}$, van de Waarenburg MP, Stehouwer CD, Schalkwijk CG. Measurement of pentosidine in human plasma protein by a single-column highperformance liquid chromatography method with fluorescence detection. Journal of chromatography. B, Analytical technologies in the biomedical and life sciences. 2009;877:610-614

26. Baumann M, Stehouwer C, Scheijen J, Heemann U, Struijker Boudier $H$, Schalkwijk C. N epsilon-(carboxymethyl)lysine during the early development of hypertension. Annals of the New York Academy of Sciences. 2008;1126:201-204

27. Hartog JW, Voors AA, Schalkwijk CG, Scheijen J, Smilde TD, Damman K, Bakker SJ, Smit AJ, van Veldhuisen DJ. Clinical and prognostic value of advanced glycation endproducts in chronic heart failure. European heart journal. 2007;28:2879-2885

28. Stolk LM, Scheijen JL. Urine adulteration with denture-cleaning tablets. Journal of analytical toxicology. 1997;21:403 


\section{Dankwoord}

Bloed, zweet en tranen 



\section{Bloed, zweet en tranen}

Anders dan de titel doet vermoeden heb ik met heel veel plezier aan dit proefschrift gewerkt. ledere keer opnieuw zag ik het als een uitdaging om een wetenschappelijke vraag met enthousiasme te beantwoorden. Ik kan mij geen leukere baan voorstellen. De reden dat ik dit alles met zo veel plezier heb gedaan (en doe) komt doordat ik werkte (en werk) in een team met geweldige collega's en promotores. Een aantal van hen wil ik hier in het bijzonder bedanken.

Beste Casper ${ }^{1}$, het zal ergens halverwege 2005 geweest zijn dat wij elkaar voor het eerst ontmoetten. Ik werkte toen bij het laboratorium voor Erfelijke Metabole ziekten en jij kwam met de vraag of wij aldaar advanced glycation endproducts konden meten. Ik had er nog nooit van gehoord maar jij wist ons al snel te overtuigen van het belang hiervan. Toen al werd mij duidelijk dat jij een gedreven wetenschapper bent met heel veel kennis, humor en enthousiasme. Op dat moment wist ik nog niet dat ik drie jaar later, samen met jou, naar het Bioanalysis congres in Warwick (Exeter University) zou gaan, om daar de methode, voor het bepalen van CM L en CEL, te presenteren. Wat was ik trots!

Inmiddels werk ik al meer dan acht jaar voor jou en heb ik veel respect en bewondering voor de manier waarop jij onze groep leidt. Ik wil je bedanken voor de vrijheid en kansen die je mij hebt gegeven. Het is een grote eer om voor je te werken en nu ook onder jouw supervisie te mogen promoveren. Ik kan me geen betere "baas" en promotor wensen! Bedankt dat ik bij je thuis mocht komen voor een goed gesprek en lekker eten als ook voor onze trip naar het noorden voor het kopen van je lang gewenste Saab cabrio!

Daarnaast wil ik ook je vrouw Hermine bedanken voor haar warme gastvrijheid en gezelligheid tijdens de vele lab-uitjes, barbecues en kerstdiners.

Beste Coen $^{2}$, toen je mij vroeg (tijdens een kerstborrel) of ik wilde gaan promoveren dacht ik "dat je een slok teveel op had". M aar al snel werd mij duidelijk dat je serieus was. Ik vond het een enorme eer en vertelde je dat ik dat fantastisch zou vinden.

Er zijn al veel superlatieven gebruikt om jou als persoon en promotor te beschrijven. Ik kan niet anders dan mij aansluiten bij alles wat er reeds over jou is gezegd. Je wetenschappelijke en algemene kennis lijken bijna oneindig en de manier waarop jij moeilijke (wetenschappelijke) problemen weet te vereenvoudigen tot een paar zinnen vind ik ongelofelijk en tegelijkertijd zeer inspirerend.

Ik wil jou bedanken dat je me deze kans hebt gegeven maar ook voor de tijd die je hebt genomen mijn zeer beperkte statistische kennis bij te spijkeren en samen te brainstormen over de hoofdstukken van mijn proefschrift. Ik ben trots dat jij één van mijn promotores bent!

Beste leden van de beoordelingscommissie; Prof. dr. Vincenzo Fogliano, Prof. dr. Tilman M. Hackeng, Prof. dr. Ron M .A. Heeren, Prof. dr. Hans W.M. Niessen en Prof. dr. Antoon Opperhuizen, bedankt voor het kritisch lezen en beoordelen van mijn proefschrift. 
De volgende collega's wil ik graag, in alfabetische volgorde, bedanken:

Beste Boy, wij delen de voorliefde voor mooie auto's (o.a. Saab), carnaval en de Limburgse taal. Op wetenschappelijk vlak hebben zich onze paden niet vaak gekruist maar desondanks kan ik zeggen dat je een fijne collega bent. Bedankt voor de leuke gesprekken en dat je mij hebt opgenomen in het selecte UIL+gezelschap.

Beste (lieve) Dionne, mannen die zeggen dat vrouwen geen gevoel voor humor hebben zouden eens een dag met jou moeten samenwerken. Wat kun jij gevat uit de hoek komen zeg! De congressen samen (die in Tokyo was natuurlijk de klapper!) waren niet alleen wetenschappelijk een succes maar ook ontzettend gezellig. Dit was voornamelijk aan jouw aanwezigheid te danken. Jij verzorgde de reizen en hotels tot in de puntjes en wist de groep feilloos van A naar B te leiden. Dit alles met de nodige humor en gezelligheid. Dat je een zeer waardevolle wetenschapper bent heb je bewezen met je prachtige proefschrift vol met uitstekende publicaties (een tweetal van jouw hand in dit proefschrift!). Ik mis je aanwezigheid, je humor en gezelligheid op ons lab en wens je heel veel succes met je verdere carrière!

Beste Kamergenoten, beste Kristiaan, je aparte gevoel voor humor heeft mijn wenkbrauwen vaak doen fronsen en je directheid deed mij vaak twijfelen over je nationaliteit (is het nou een Hollander of een Belg?). Desondanks, was jij een waardevolle kamergenoot en was er altijd wat te lachen! Bedankt voor al je adviezen en wetenschappelijke input.

Beste Mathias, de andere Belg (né Anvers) op mijn kamer, jij wist mijn grote mond vaak in toom te houden. "Zeg Jean, zou je dit nou wel doen?" of "Dat vind iek iek echt niet kunnen." waren uitspraken van jou die mijn soms impulsieve karakter afremden. En dan te bedenken dat je 20 jaar jonger bent, je zou eigenlijk $U$ tegen mij moeten zeggen! We gingen (vaak) samen naar de fitness en op stap tot in de kleine uurtjes. Je bent een geweldige collega!

Beste (lieve) Katrien, ik mocht jouw paranimf zijn en weet nog dat ik zenuwachtiger was dan jijzelf. Jij bent ongelofelijk gestructureerd, een geweldig onderzoeker en een meer dan prettige collega. Je nuchtere kijk op dingen en je droge gevoel voor humor heb ik altijd kunnen waarderen.

Beste (lieve) Marjo, mijn partner in crime, wat hebben we veel gelachen maar ook heel veel bergen werk verzet. Niets was jou teveel! Je kwam weekenden terug om ervoor te zorgen dat de tandem MS twentyfourseven bleef analyseren. Zo heb jij ook bijgedragen aan de ontwikkeling van nieuwe methoden, je had goede ideeën en suggesties. De meest briljante ideeën kwamen gek genoeg op vrijdagmiddagen, meer dan één keer hadden we juist dan succes! Ons beider enthousiasme werkte aanstekelijk en we motiveerden elkaar wanneer het eens wat minder ging. Bedankt M arjo, maar dan ook echt voor ALLES! 
Beste Marleen, je zult wel af en toe last hebben gehad van dat lawaai van die drie kerels naast je. Daar wil ik mij bij dezen, ook namens mijn kamergenoten, voor verontschuldigen. Je deur stond altijd open voor advies en daar maakte ik dan ook graag gebruik van, bedankt daarvoor.

Beste (lieve) Monica, lief en leed hebben wij samen gedeeld. In het begin dacht ik, "...die is van Parkstad, dat is niks..." maar ik kan nu zeggen dat ik je ook onder het kopje vrienden had kunnen noemen. De keren dat we samen op stap gingen zijn niet op 1 hand te tellen (ook niet op 2) alsook de koppen koffie en de gesprekken over de liefde. Ik ben blij dat ik jou en je familie heb leren kennen en hoop dat we nog lang vrienden zullen blijven. Ik wens je heel veel succes met je verdere carrière!

Beste Nordin, ik mocht jouw paranimf zijn, wat een eer was dat! Jij bent echt één van de meest intelligente mensen die ik ken (en Coen natuurlijk). Dat jij Cum Laude promoveerde was dan ook geen verassing. Ik mag wel zeggen dat de publicaties, die wij samen schreven, voornamelijk door jouw input naar een hoger niveau gebracht werden. Ik wil je daarvoor bedanken. Ik ben blij dat ik niet alleen jou, maar ook je zussen, Irmelin en Noraly, je vriendin Burcu, alsook je ouders heb mogen leren kennen. Wat een leuke en lieve familie heb jij! Ik hoop dat we elkaar in de toekomst nog vaak mogen zien.

Beste Stijn, wij delen onze voorliefde voor mooie spullen en auto's (ik heb dan wel een iets kleiner budget, maar goed we delen de voorliefde). Ons congres in Wenen was legendarisch. Tot diep in de nacht (bijna ochtend) genoten wij van diverse WodkaRedbull's ergens onder de grond in één of andere vage club. Het was de laatste avond, slapen konden we in de taxi en in het vliegtuig en dat deden we. Je bent een echte dokter (Internist), hoewel ik soms denk, gezien je kleding stijl, dat je beter bij chirurgie had kunnen werken. Ik vind het jammer dat je niet meer op onze afdeling rondloopt, ik mis je droge humor en kritische blik op sommige aspecten van het leven. Stijn, succes met alles!

Daarnaast wil ik ook graag mijn andere collega's bedanken. ledereen staat onvoorwaardelijk voor je klaar en is altijd in voor gezelligheid; Nynke, Mitchell, Ying, Paul, Petra, Wenjie, Vicky, Margee, Maria, José, Yvo, Suzan, Jos, Adriaan, Birgit, Daisy, Tiny (!), Jo, Eddy, Maurice, Eugene, Mark, Roland en Simon.

Beste co-auteurs, bedankt voor jullie bijdrage aan mijn manuscripten; Prof. dr. Coen D.A. Stehouwer, Prof. dr. Casper G. Schalkwijk, Dr. Nordin M.J. Hanssen, Drs. Marjo P.H. van de Waarenburg, Dr. Dionne E.M. M aessen, Dr. Daisy M .A.E. Jonkers, M Sc. Egbert Clevers, Dr. Lian Engelen, Prof. dr. Ir. Pieter C. Dagnelie, Prof. dr. Fred Brouns, Dr. Carla J. Van der Kallen, Dr. Marleen M. van Greevenbroek en Prof. dr. ir. Edith J. M. Feskens

Beste oud collega's, ook jullie wil ik bedanken voor de gezelligheid en alles wat ik van jullie geleerd heb; Jack, Leo, Karin, Yvo, Jos, Bert, Harry, Loes, Henk (laboratorium Klinische Farmacie \& Toxicologie), Albert, Leo, Jaap, Jörgen, Jan, Huub, Marc, Marjon, 
Karin, Ivo, Genna (laboratorium Erfelijke Metabole ziekten), Mick, Hans, Steven, Cees (Algemene Heelkunde), Samefko, Mark, Daniel, Johanna, Bas, Lian, Marcelle, Roel, Olaf, Amy, Leon, Matthijs, Rianne, Jolanda, Mateusz, Elisabeth (bedankt dat ik je paranimf mocht zijn!), Teba, Dennis, Barry, Hanneke, Pieter (Interne Geneeskunde).

Ik wil ook graag de mensen van de firma Waters bedanken voor hun support; Ad, Perry, Mike, Koen en Fred.

Beste (lieve) vrienden, jullie wil ik ook graag bedanken voor de mooie momenten die we samen hadden (en hebben). Deze momenten zorgde (en zorgen) ervoor dat ik elke dag met veel plezier en energie naar mijn werk ging (en ga)!

Beste paranimfen, Dyon, Armand, daar staan we dan gedrieën naast elkaar, broers, trots op de naam Scheijen! Bedankt dat jullie er altijd voor mij zijn!

Lieve pap en mam, ik kan een boek schrijven over hoe dankbaar ik ben voor de kansen die jullie ons (mijn broers en ik) gegeven hebben. Jullie zijn een onvoorwaardelijke steun voor ons alle drie! Ik kan mij geen betere ouders wensen, ik hou van jullie.

Lieve Giel en Pie, mijn jongens, mijn trots! Dit proefschrift is voor jullie en hoop dat het een inspiratie zal zijn voor de toekomst.

1. Prof. dr. C.G. Schalkwijk, bijzonder hoogleraar Experimentele Interne Geneeskunde, hoofd Algemene Interne Geneeskunde (Universiteit Maastricht)

2. Prof. dr. C.D.A. Stehouwer, Internist (M aastricht Universitair M edisch Centrum plus), hoofd Interne Geneeskunde, bijzonder hoogleraar Interne Geneeskunde 


\section{Foods \& Polaroid}

Chapter title pages

Original Polaroid \& Impossible Project film 



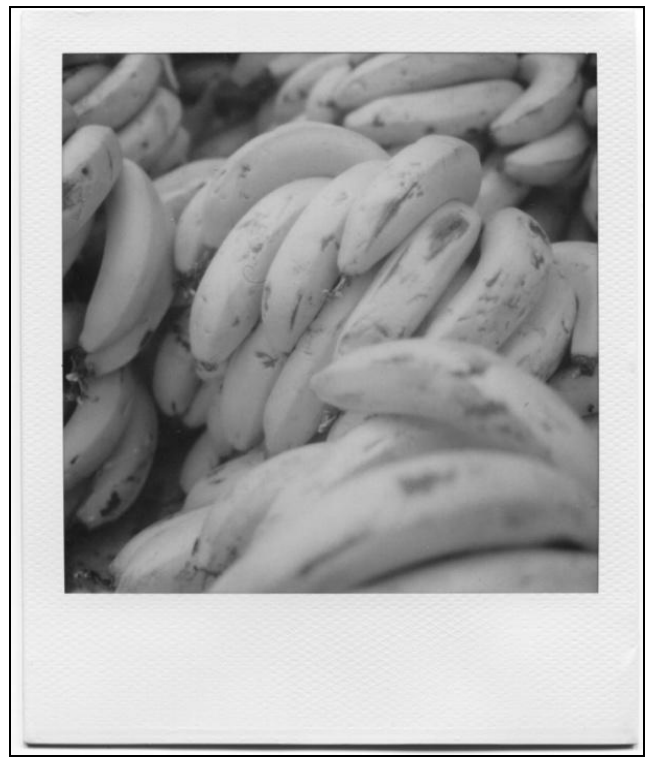

\section{BANANA}

Film: Original Polaroid 600 with ND filter

Camera: Polaroid SX-70 Sonar AF

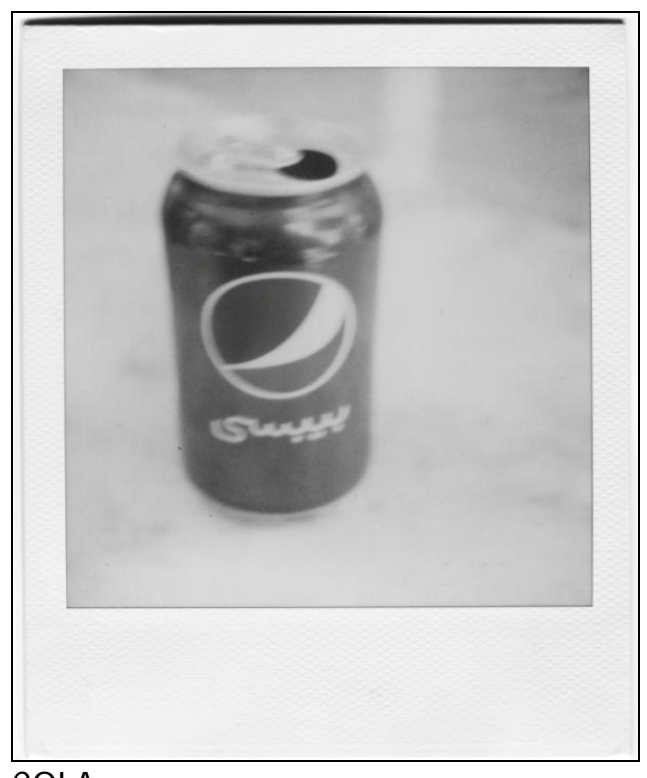

\section{COLA}

Film: Original Polaroid 600 with ND filter

Camera: Polaroid SX-70 Original

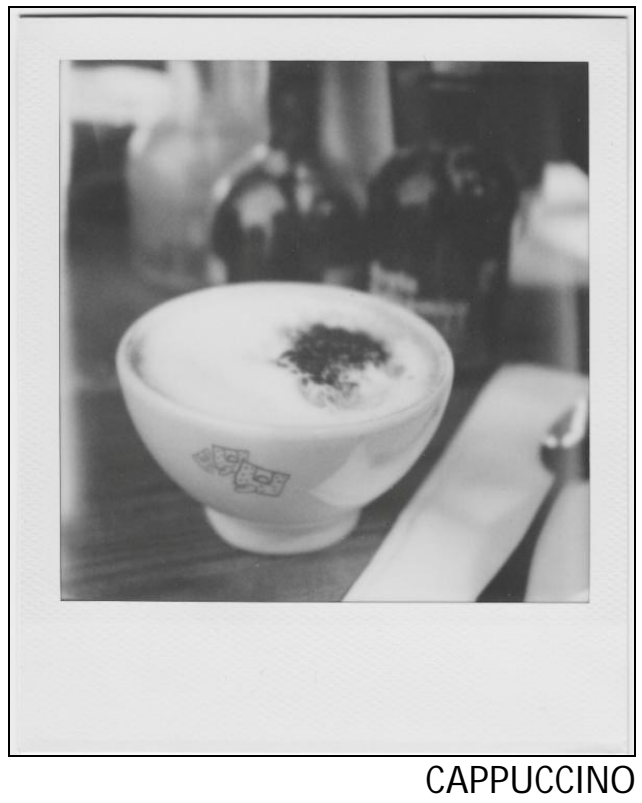

Film: Original Polaroid 600 with ND filter Camera: Polaroid SX-70 Original

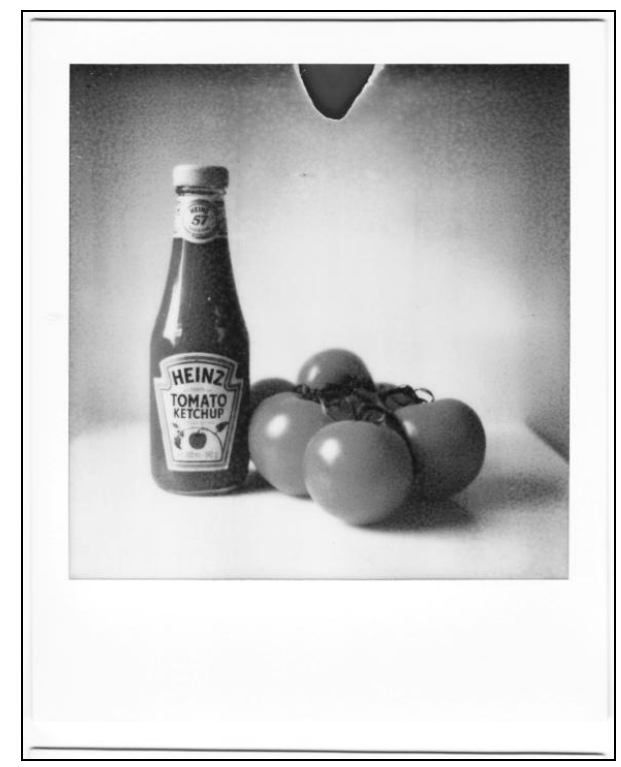

HEINZTOMATO KETCHUP

Film: PX-70 Color Shade (Impossible Project) Camera: Polaroid SX-70 Alpha 1 model 2 


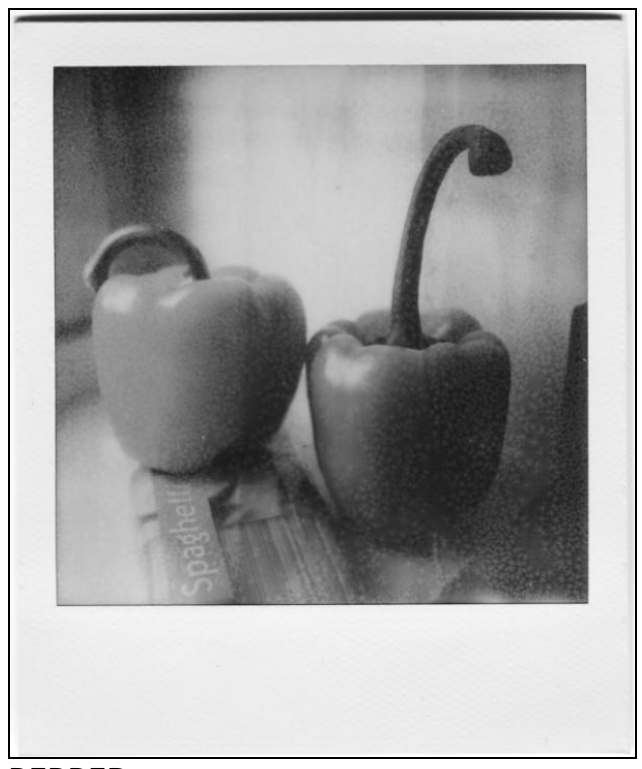

\section{PEPPER}

Film: PX-70 Color Shade (Impossible Project)

Camera: Polaroid SX-70 Original

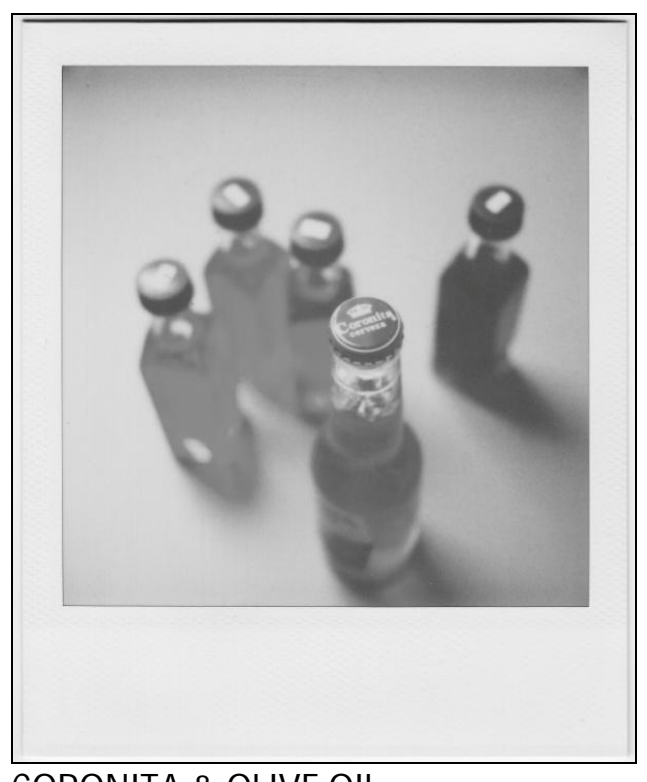

CORONITA \& OLIVE OIL

Film: Original Polaroid 600 with ND filter

Camera: Polaroid SX-70 Alpha 1 model 2

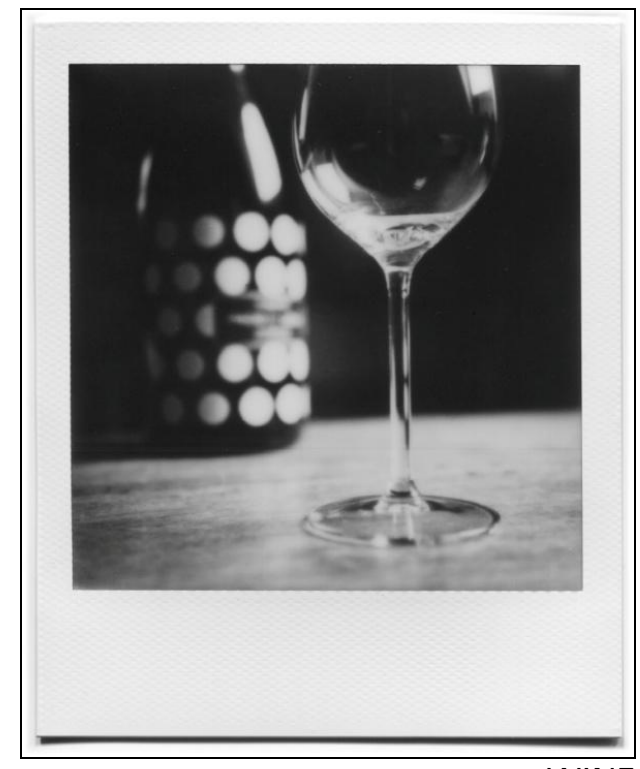

WINE

Film: PX-70 Color Shade (Impossible Project) Camera: Polaroid SX-70 Original

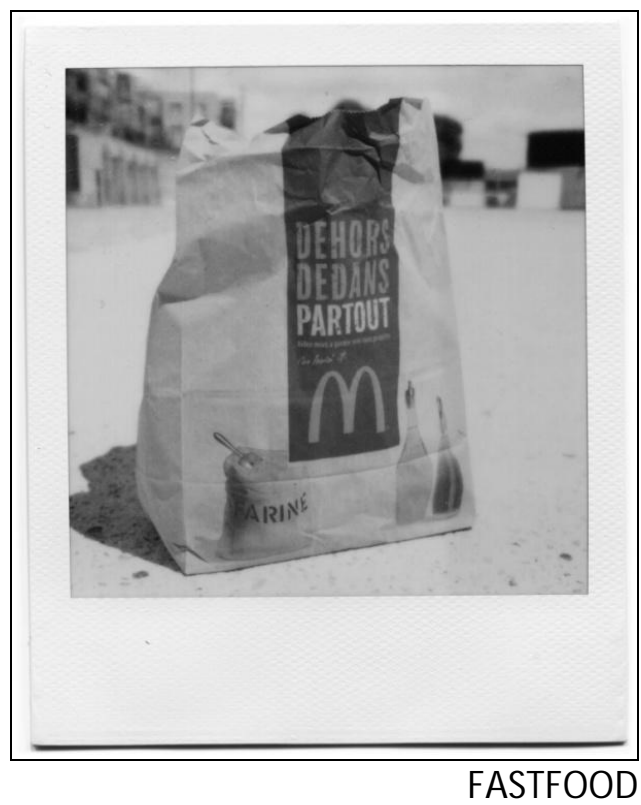

Film: Original Polaroid 600 with ND filter Camera: Polaroid SX-70 Original 


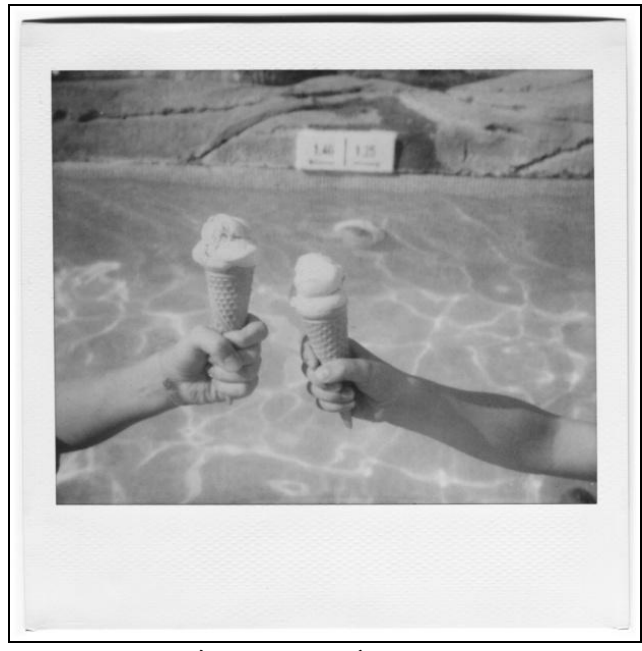

ICE CREAM (Giel \& Pie)

Film: Polaroid Image Softtone

Camera: Polaroid Image System

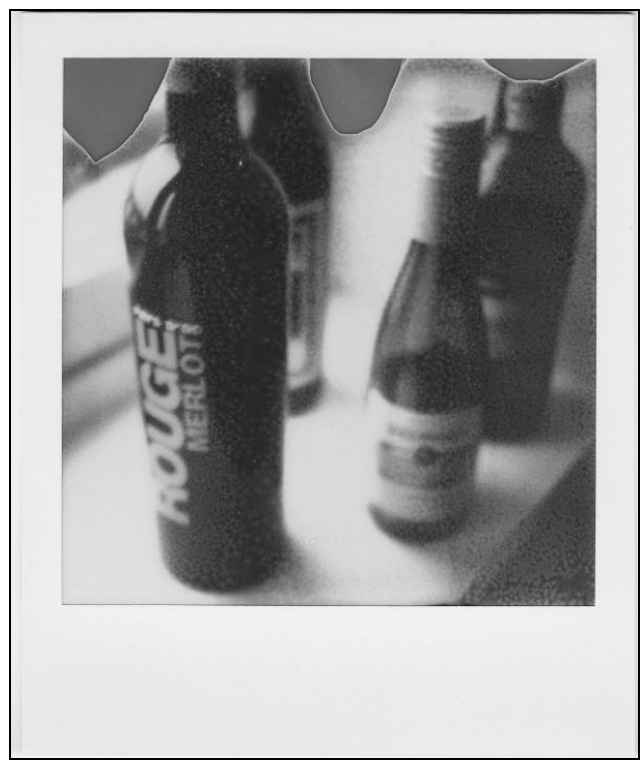

\section{MERLOT}

Film: PX-70 Color Shade (Impossible Project) Camera: Polaroid SX-70 Original

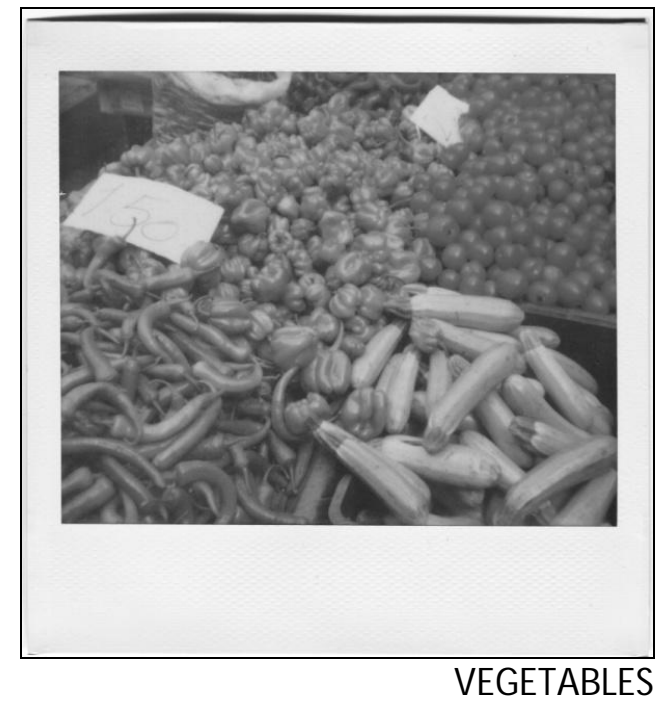

Film: Polaroid Image Softtone Camera: Polaroid Image System

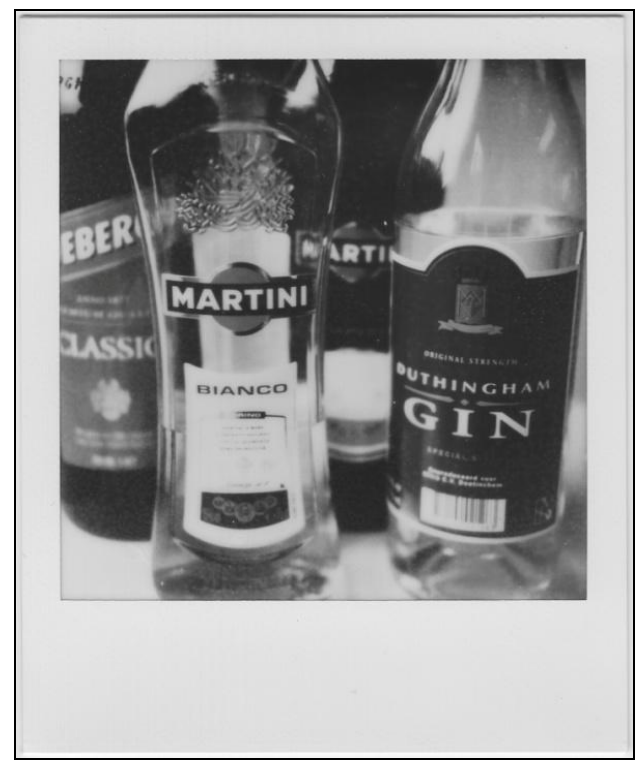

MARTINI \& GIN

Film: PX-70 Color Shade (Impossible Project) Camera: Polaroid SX-70 Alpha 1 model 2 